\title{
Estimating Impacts of Diesel Fuel Reformulation with Vector-based Blending
}

\section{December 2002}

Prepared by

G. R. Hadder

Oak Ridge National Laboratory

R.W. Crawford

R.W. Crawford Energy Systems

H. T. McAdams

AccaMath Services

B. D. McNutt

U.S. Department of Energy 


\title{
DOCUMENT AVAILABILITY
}

Reports produced after January 1, 1996, are generally available free via the U.S. Department of Energy (DOE) Information Bridge:

Web site: http://www.osti.gov/bridge

Reports produced before January 1, 1996, may be purchased by members of the public from the following source:

\author{
National Technical Information Service \\ 5285 Port Royal Road \\ Springfield, VA 22161 \\ Telephone: 703-605-6000 (1-800-553-6847) \\ TDD: $703-487-4639$ \\ Fax: 703-605-6900 \\ E-mail: info@ntis.fedworld.gov \\ Web site: http://www.ntis.gov/support/ordernowabout.htm
}

Reports are available to DOE employees, DOE contractors, Energy Technology Data Exchange (ETDE) representatives, and International Nuclear Information System (INIS) representatives from the following source:

Office of Scientific and Technical Information

P.O. Box 62

Oak Ridge, TN 37831

Telephone: 865-576-8401

Fax: $865-576-5728$

E-mail: reports@adonis.osti.gov

Web site: http://www.osti.gov/contact.html

This report was prepared as an account of work sponsored by an agency of the United States Government. Neither the United States government nor any agency thereof, nor any of their employees, makes any warranty, express or implied, or assumes any legal liability or responsibility for the accuracy, completeness, or usefulness of any information, apparatus, product, or process disclosed, or represents that its use would not infringe privately owned rights. Reference herein to any specific commercial product, process, or service by trade name, trademark, manufacturer, or otherwise, does not necessarily constitute or imply its endorsement, recommendation, or favoring by the United States Government or any agency thereof. The views and opinions of authors expressed herein do not necessarily state or reflect those of the United States Government or any agency thereof. 


\title{
ESTIMATING IMPACTS OF DIESEL FUEL REFORMULATION WITH VECTOR-BASED BLENDING
}

\author{
G. R. Hadder \\ Transportation Technology Group \\ Oak Ridge National Laboratory \\ Oak Ridge, Tennessee \\ R.W. Crawford \\ R.W. Crawford Energy Systems \\ Tucson, Arizona \\ H.T. McAdams \\ AccaMath Services \\ Carrollton, Illinois

\section{B.D. McNutt} \\ U.S. Department of Energy \\ Office of Policy and International Affairs \\ Washington, DC \\ December 2002 \\ Prepared for \\ U.S. Department of Energy Offices of \\ Policy and International Affairs \\ Energy Efficiency and Renewable Energy \\ Fossil Energy \\ Prepared by the \\ OAK RIDGE NATIONAL LABORATORY \\ P.O. Box 2008 \\ Oak Ridge, Tennessee 37831-6285 \\ managed by \\ UT-Battelle, LLC \\ for the \\ U.S. DEPARTMENT OF ENERGY \\ under contract DE-AC05-00OR22725
}





\section{TABLE OF CONTENTS}

Page

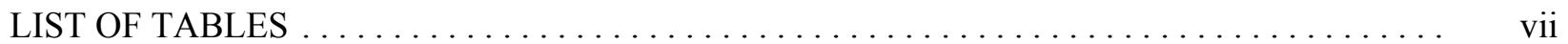

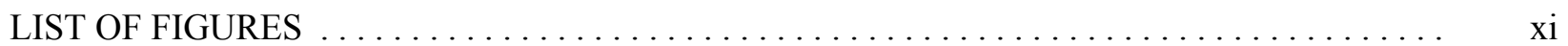

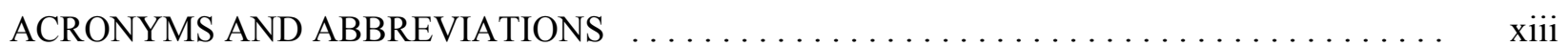

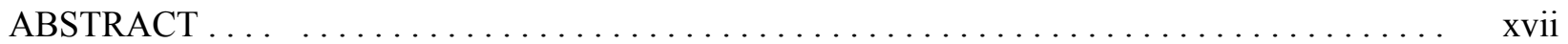

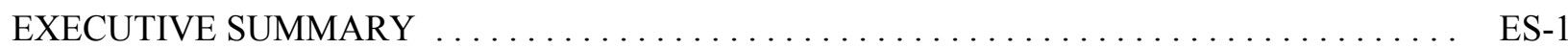

ES.1 OBJECTIVES AND KEY FINDINGS $\ldots \ldots \ldots \ldots \ldots \ldots \ldots \ldots \ldots \ldots \ldots \ldots$ ES-1

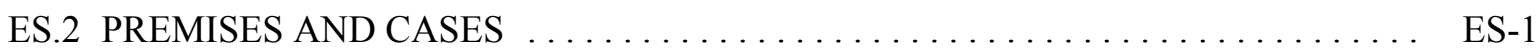

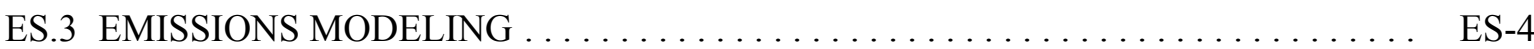

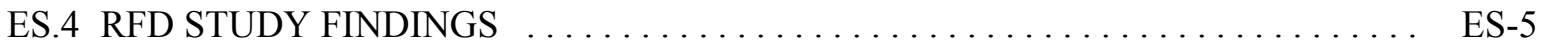

PART I: THE REFINERY STUDY

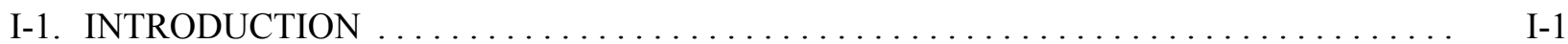

I-2. THE ORNL REFINERY YIELD MODEL $\ldots \ldots \ldots \ldots \ldots \ldots \ldots \ldots \ldots \ldots \ldots \ldots \ldots \ldots$ I-5

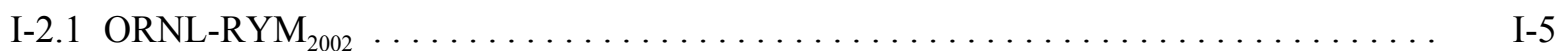

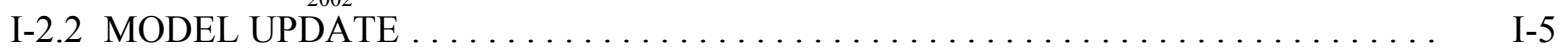

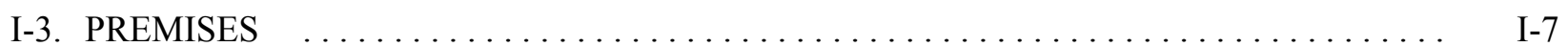

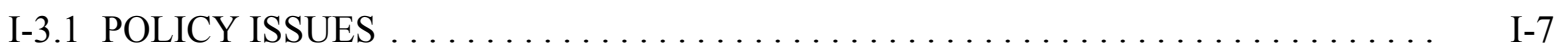

I-3.2 STUDY PERIOD AND GEOGRAPHIC AREA $\ldots \ldots \ldots \ldots \ldots \ldots \ldots \ldots \ldots \ldots$ I-8

I-3.3 TECHNICAL PREMISES $\ldots \ldots \ldots \ldots \ldots \ldots \ldots \ldots \ldots \ldots \ldots \ldots \ldots \ldots \ldots \ldots \ldots \ldots \ldots$

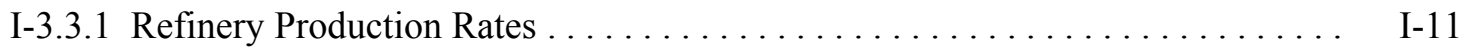

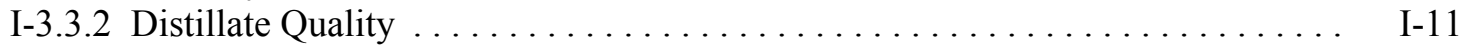

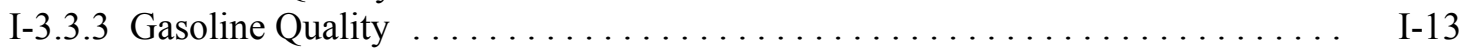

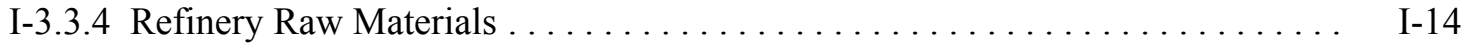

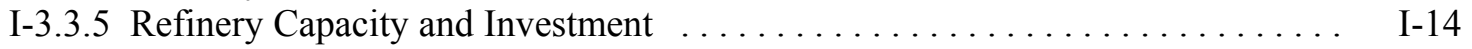

I-3.3.6 Product Revenue and Raw Material Costs $\ldots \ldots \ldots \ldots \ldots \ldots \ldots \ldots \ldots \ldots \ldots \ldots \ldots \ldots \ldots$

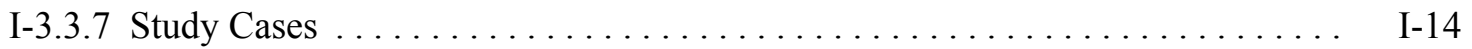

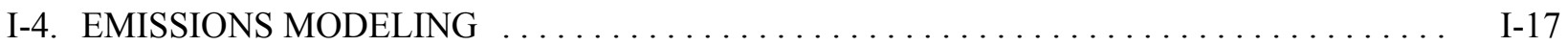

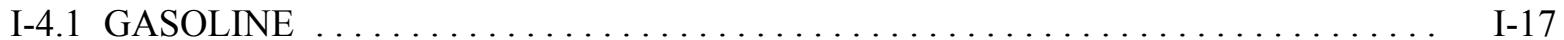

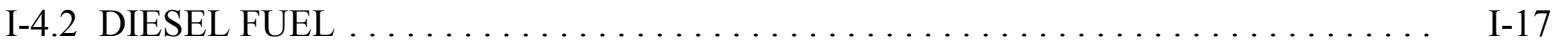

I-4.3 DIESEL FUEL EMISSIONS MODELING CONCERNS $\ldots \ldots \ldots \ldots \ldots \ldots \ldots \ldots$ I-18

I-5. CALIBRATION AND DEMONSTRATION $\ldots \ldots \ldots \ldots \ldots \ldots \ldots \ldots \ldots \ldots \ldots \ldots \ldots \ldots \ldots$

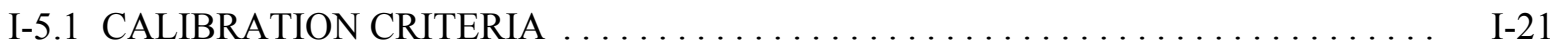

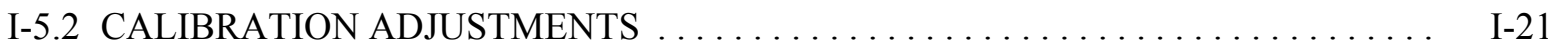

I-5.3 CALIBRATION RESULTS $\ldots \ldots \ldots \ldots \ldots \ldots \ldots \ldots \ldots \ldots \ldots \ldots \ldots \ldots \ldots \ldots \ldots \ldots \ldots \ldots \ldots .22$ 
I-6. IMPACTS OF DIESEL FUEL REFORMULATION IN PADD II REFINERIES . . . . . . . I-27

I-6.1 BASE CASE 1: LOW SULFUR DIESEL FUEL $\ldots \ldots \ldots \ldots \ldots \ldots \ldots \ldots \ldots \ldots$ I-27

I-6.2 CASE 1.1: PARALLEL INVESTMENT AND VEHICLE PERFORMANCE RFD . . I I-54

I-6.3 CASE 1.2: PARALLEL INVESTMENT AND EMISSIONS REDUCTION RFD . . . I I-54

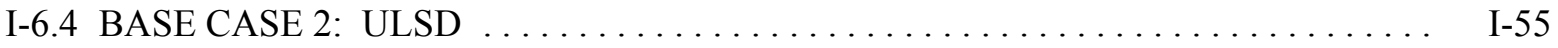

I-6.5 CASE 2.1: SEQUENTIAL INVESTMENT AND VEHICLE PERFORMANCE RFD . . . . . . . . . . . . . . . $\ldots \ldots \ldots \ldots \ldots \ldots$ I-56

I-6.6 CASE 2.2: SEQUENTIAL INVESTMENT AND EMISSIONS

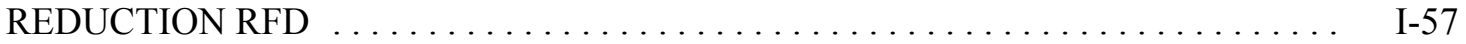

I-6.7 EMISSIONS SPECIFICATION BENEFITS $\ldots \ldots \ldots \ldots \ldots \ldots \ldots \ldots \ldots \ldots \ldots \ldots \ldots \ldots \ldots \ldots$

I-6.8 OFF-ROAD SULFUR $\ldots \ldots \ldots \ldots \ldots \ldots \ldots \ldots \ldots \ldots \ldots \ldots \ldots \ldots \ldots \ldots \ldots \ldots \ldots \ldots \ldots \ldots$

I-7. IMPACT OF DIESEL FUEL REFORMULATION ON DEMAND FOR CANADIAN SYNTHETIC CRUDE $\ldots \ldots \ldots \ldots \ldots \ldots \ldots \ldots \ldots \ldots \ldots \ldots \ldots \ldots \ldots \ldots \ldots \ldots \ldots \ldots$

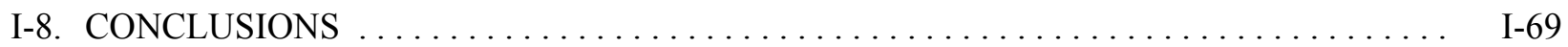

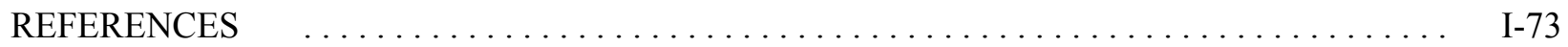

APPENDIX I-A: ENSYS ENERGY \& SYSTEMS, INC., PROGRESS REPORT NUMBER 1 TECHNOLOGY AND FEATURES ENHANCEMENTS TO THE ORNL

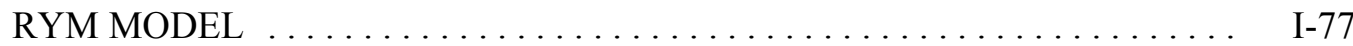

APPENDIX I-B: ENSYS ENERGY \& SYSTEMS, INC., PROGRESS REPORT NUMBER 2 TECHNOLOGY AND FEATURES ENHANCEMENTS TO THE ORNL

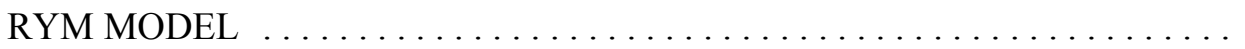

APPENDIX I-C: API COMMENTS ON RESEARCH PROPOSAL "ESTIMATING IMPACTS OF DIESEL FUEL REFORMULATION WITH VECTOR-BASED BLENDING"

APPENDIX I-D: API COMMENTS ON DOE RFD STUDY $\mathrm{I}-105$

\section{PART II: DIESEL FUEL EMISSIONS MODELS FOR THE ORNL REFINERY YIELD MODEL}

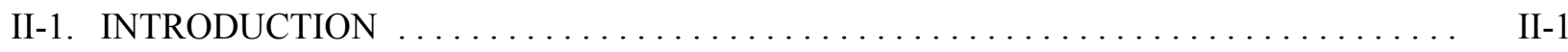

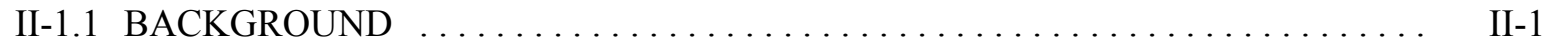

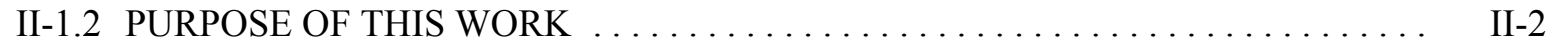

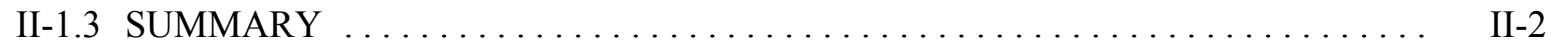

II-1.4 ORGANIZATION OF THE REPORT $\ldots \ldots \ldots \ldots \ldots \ldots \ldots \ldots \ldots \ldots \ldots \ldots \ldots \ldots \ldots \ldots \ldots$

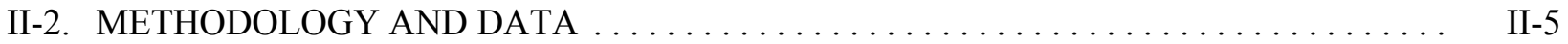

II-2.1 DIESEL FUEL AND EMISSIONS DATABASE $\ldots \ldots \ldots \ldots \ldots \ldots \ldots \ldots \ldots$ II-5

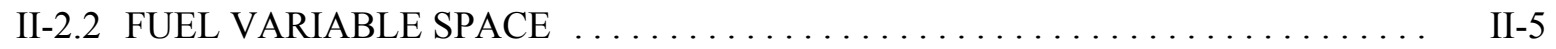

II-2.3 METHODOLOGY FOR MODEL DEVELOPMENT $\ldots \ldots \ldots \ldots \ldots \ldots \ldots \ldots \ldots$ II-7 
II-3. EIGENVECTOR EMISSIONS MODELS $\ldots \ldots \ldots \ldots \ldots \ldots \ldots \ldots \ldots \ldots \ldots \ldots \ldots \ldots$ II-11

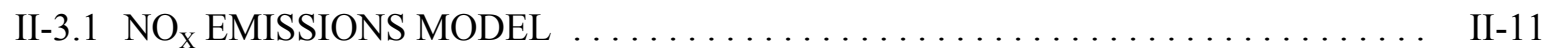

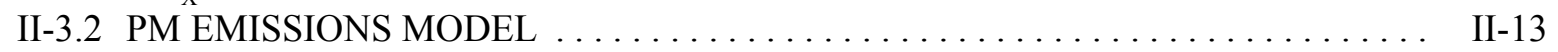

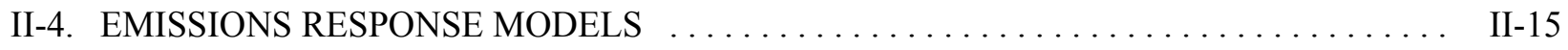

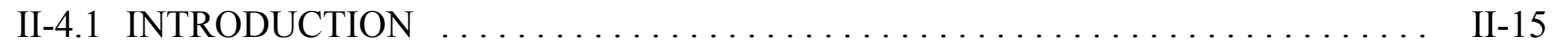

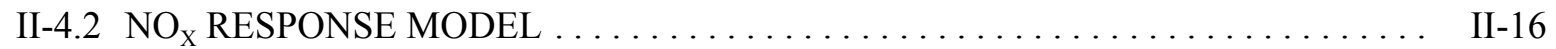

II-4.3 PM RESPONSE MODEL $\ldots \ldots \ldots \ldots \ldots \ldots \ldots \ldots \ldots \ldots \ldots \ldots \ldots \ldots \ldots \ldots \ldots \ldots \ldots \ldots \ldots \ldots \ldots \ldots$

II-5. IMPLEMENTATION IN RYM . . . . . . . . . . . . . . . . . . . . . II-29

II-5.1 REPRESENTING TECHNOLOGICAL CHANGE $\ldots \ldots \ldots \ldots \ldots \ldots \ldots \ldots \ldots \ldots$ II-29

II-5.2 IMPLEMENTATION IN RYM $\ldots \ldots \ldots \ldots \ldots \ldots \ldots \ldots \ldots \ldots \ldots \ldots \ldots \ldots \ldots \ldots \ldots \ldots \ldots \ldots$

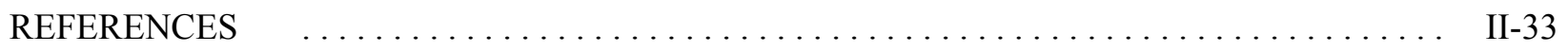

APPENDIX II-A: STATISTICAL RESULTS FOR NO $\mathrm{NO}_{\mathrm{X}}$ EMISSIONS MODEL $\ldots \ldots \ldots \ldots$ II-35

APPENDIX II-B: STATISTICAL RESULTS FOR PM EMISSIONS MODEL $\ldots \ldots \ldots \ldots \ldots$ II-41

APPENDIX II-C: PEER REVIEW COMMENTS AND REPLY $\ldots \ldots \ldots \ldots \ldots \ldots \ldots \ldots \ldots . \quad$ II-47 



\section{LIST OF TABLES}

Table

Page

ES-1 On-road No. 2 diesel fuel regulations and timing $\ldots \ldots \ldots \ldots \ldots \ldots \ldots \ldots \ldots \ldots$

ES-2 Diesel fuel reformulation case studies for U.S. Midwestern refineries

in summer 2010

ES-4

ES-3 Diesel fuel reformulation study findings $\ldots \ldots \ldots \ldots \ldots \ldots \ldots \ldots \ldots \ldots \ldots$

\section{PART I: THE REFINERY STUDY}

1.1 On-road No. 2 diesel fuel regulations and timing $\ldots \ldots \ldots \ldots \ldots \ldots \ldots \ldots \ldots \ldots \ldots \ldots \ldots \ldots$

3.1 PADD II raw materials and products for year 2010 summer $\ldots \ldots \ldots \ldots \ldots \ldots \ldots \ldots$ I-9

3.2 PADD II pre-investment process capacity for year 2010 summer $\ldots \ldots \ldots \ldots \ldots \ldots \ldots \ldots$ I-10

3.3 Diesel fuel reformulation case studies for summer $2010 \ldots \ldots \ldots \ldots \ldots \ldots \ldots \ldots \ldots$ I-12

5.1 Crude oil quality calibration demonstration for PADD II summer $\ldots \ldots \ldots \ldots \ldots \ldots \ldots$ I-22

5.2 Process unit utilization calibration demonstration for PADD II summer $\ldots \ldots \ldots \ldots \ldots$ I-22

5.3 Reformulated gasoline calibration demonstration for PADD II summer $\ldots \ldots \ldots \ldots \ldots \ldots$ I-23

5.4 Conventional gasoline calibration demonstration for PADD II summer $\ldots \ldots \ldots \ldots \ldots \ldots$ I-24

5.5 Distillate calibration demonstration for PADD II summer $\ldots \ldots \ldots \ldots \ldots \ldots \ldots \ldots \ldots$ I-25

6.1 Diesel fuel reformulation case studies for PADD II in summer $2010 \ldots \ldots \ldots \ldots \ldots \ldots$ I-27

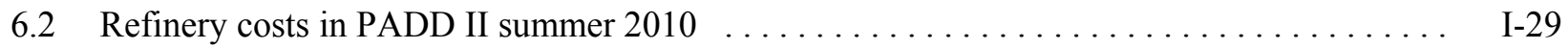

6.3 Properties of diesel fuels in PADD II summer $2010 \ldots \ldots \ldots \ldots \ldots \ldots \ldots \ldots \ldots \ldots \ldots$

6.4 Properties of on-road diesel fuels, by desulfurization (DeS) technology, in PADD II

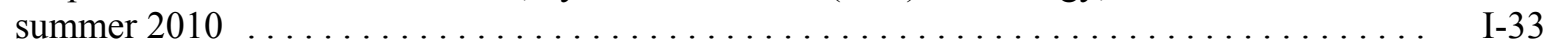

6.5 Blendstocks of diesel fuels in PADD II summer $2010 \ldots \ldots \ldots \ldots \ldots \ldots \ldots \ldots \ldots \ldots$

6.6 Properties of gasolines in PADD II summer $2010 \ldots \ldots \ldots \ldots \ldots \ldots \ldots \ldots \ldots \ldots \ldots \ldots$

6.7 Blendstocks of gasolines in PADD II summer $2010 \ldots \ldots \ldots \ldots \ldots \ldots \ldots \ldots \ldots \ldots$ I-43

6.8 Pooled gasoline blendstocks in PADD II summer $2010 \ldots \ldots \ldots \ldots \ldots \ldots \ldots \ldots \ldots$

6.9 Refinery volume balance in PADD II summer $2010 \ldots \ldots \ldots \ldots \ldots \ldots \ldots \ldots \ldots \ldots \ldots$ 
6.10 Hydrogen balance for PADD II refineries $\ldots \ldots \ldots \ldots \ldots \ldots \ldots \ldots \ldots \ldots \ldots \ldots \ldots \ldots$

6.11 Process capacity expansions and additions in PADD II summer $2010 \ldots \ldots \ldots \ldots \ldots \ldots$ I-50

6.12 Cost of process capacity expansions and additions in PADD II summer $2010 \ldots \ldots \ldots \ldots$ I-51

6.13 Components of refinery cost changes in PADD II summer $2010 \ldots \ldots \ldots \ldots \ldots \ldots \ldots$ I-52

6.14 Quality of crude oil processed in PADD II summer $2010 \ldots \ldots \ldots \ldots \ldots \ldots \ldots \ldots \ldots$

6.15 Refinery energy use changes in PADD II summer $2010 \ldots \ldots \ldots \ldots \ldots \ldots \ldots \ldots \ldots \ldots$

6.16 Refinery costs in PADD II summer 2010 with average specifications $\ldots \ldots \ldots \ldots \ldots \ldots$ I-59

6.17 Properties of RFD, with average specifications, in PADD II summer $2010 \ldots \ldots \ldots \ldots \ldots$ I-60

6.18 Sulfur content of PADD II off-road diesel fuels $\ldots \ldots \ldots \ldots \ldots \ldots \ldots \ldots \ldots \ldots \ldots$ I-62

7.1 Comparison of crude oils in RFD study $\ldots \ldots \ldots \ldots \ldots \ldots \ldots \ldots \ldots \ldots \ldots \ldots \ldots \ldots \ldots \ldots \ldots \ldots$

7.2 Comparison of fraction properties in crude oils of RFD study

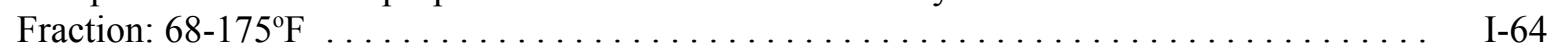

7.3 Comparison of aggregated fraction properties in crude oils of RFD study

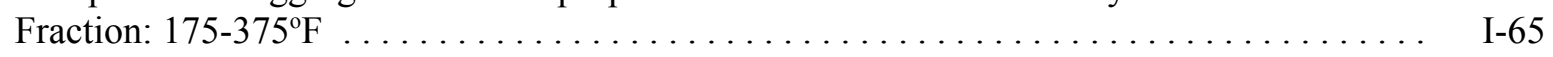

7.4 Comparison of aggregated fraction properties in crude oils of RFD study

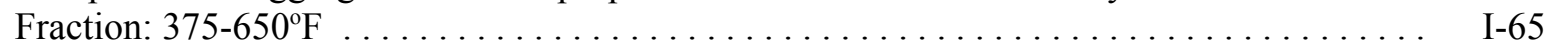

7.5 Comparison of fraction properties in crude oils of RFD study

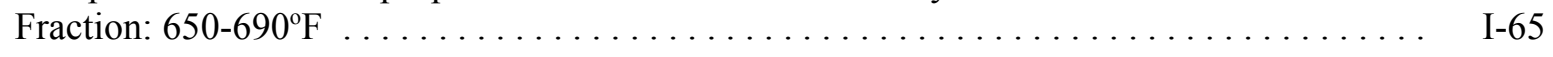

7.6 Comparison of fraction properties in crude oils of RFD study

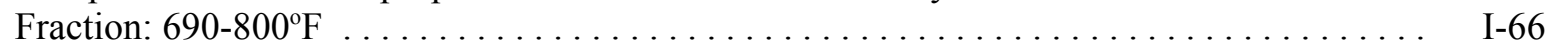

7.7 Comparison of fraction properties in crude oils of RFD study

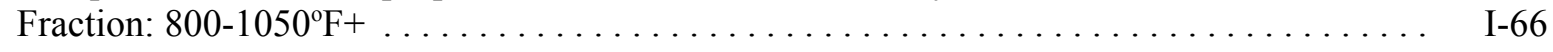

8.1 Diesel fuel reformulation study findings $\ldots \ldots \ldots \ldots \ldots \ldots \ldots \ldots \ldots \ldots \ldots \ldots \ldots \ldots \ldots \ldots$

\section{PART II: DIESEL FUEL EMISSIONS MODELS FOR THE ORNL REFINERY YIELD MODEL}

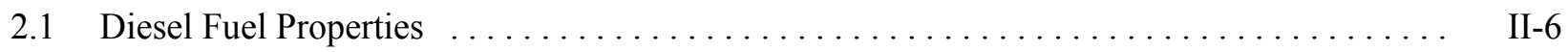

2.2 Terms Contained in Emission Models $\ldots \ldots \ldots \ldots \ldots \ldots \ldots \ldots \ldots \ldots \ldots \ldots \ldots \ldots \ldots$ 
3.1 Sum of Squares Contributions by Fuel Property for the $\mathrm{NO}_{\mathrm{x}}$ Emissions Model . . . . . . II-12

3.2 Sum of Squares Contributions by Fuel Property for the PM Emissions Model . . . . . . . . II-14

$4.1 \quad$ Relevant Range of Fuel Property Values $\ldots \ldots \ldots \ldots \ldots \ldots \ldots \ldots \ldots \ldots \ldots \ldots \ldots$

4.2 Reduced-Form $\mathrm{NO}_{\mathrm{x}}$ Response Model $\ldots \ldots \ldots \ldots \ldots \ldots \ldots \ldots \ldots \ldots \ldots \ldots \ldots \ldots$

4.3 Magnitude of Predicted $\mathrm{NO}_{\mathrm{x}}$ Emissions Response $\ldots \ldots \ldots \ldots \ldots \ldots \ldots \ldots \ldots \ldots \ldots \ldots \ldots \ldots$

4.4 Reduced-Form PM Response Model $\ldots \ldots \ldots \ldots \ldots \ldots \ldots \ldots \ldots \ldots \ldots \ldots \ldots \ldots$ II-22

4.5 Magnitude of Predicted PM Emissions Response $\ldots \ldots \ldots \ldots \ldots \ldots \ldots \ldots \ldots \ldots \ldots \ldots \ldots \ldots$

5.1 Fraction of Diesel Highway $\mathrm{NO}_{\mathrm{x}}$ Inventory by EGR System Type $\ldots \ldots \ldots \ldots \ldots \ldots$ II-30

5.2 Assumptions for the Effect of Advanced Technology Engines on Fleetwide

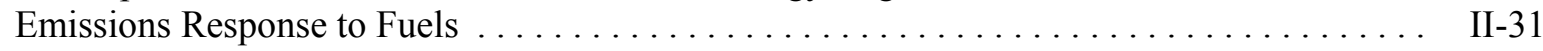





\section{LIST OF FIGURES}

Figure

Page

\section{PART I: THE REFINERY STUDY}

4.1 Comparative Performance of Regression Models $\ldots \ldots \ldots \ldots \ldots \ldots \ldots \ldots \ldots \ldots \ldots \ldots$ I-19 . . . . . . . .

7.1 ULS Diesel Increases Demand for Synthetic Crude $\ldots \ldots \ldots \ldots \ldots \ldots \ldots \ldots \ldots \ldots$

7.2 RFD Reduces Demand for Synthetic Crude at Recent Prices: Parallel Investment . . . . . . . I-67

7.3 Synthetic Crude Demand Comparisons $\ldots \ldots \ldots \ldots \ldots \ldots \ldots \ldots \ldots \ldots \ldots \ldots \ldots \ldots$

7.4 Hydroprocessing Capacity Is Related to Synthetic Crude Demand Outlook:

Parallel Investment with Vehicle Performance RFD . . . . . . . . . . . . . . . . . . . . I-68

\section{PART II: DIESEL FUEL EMISSIONS MODELS FOR THE ORNL REFINERY YIELD MODEL}

2.1 Distribution of Fuel Sulfur Content $\ldots \ldots \ldots \ldots \ldots \ldots \ldots \ldots \ldots \ldots \ldots \ldots \ldots \ldots \ldots \ldots \ldots$

3.1 Sum of Squares Contributions to $\mathrm{NO}_{x}$ Model $\ldots \ldots \ldots \ldots \ldots \ldots \ldots \ldots \ldots \ldots \ldots \ldots \ldots$

3.2 Sum of Squares Contributions to PM Model $\ldots \ldots \ldots \ldots \ldots \ldots \ldots \ldots \ldots \ldots \ldots \ldots$ II-13

$4.1 \quad \mathrm{NO}_{\mathrm{x}}$ Emissions Response to Total Cetane Number $\ldots \ldots \ldots \ldots \ldots \ldots \ldots \ldots \ldots \ldots \ldots$

$4.2 \mathrm{NO}_{\mathrm{x}}$ Emissions Response to Natural and Additized Cetane . . . . . . . . . . . . . . . II-18

$4.3 \quad \mathrm{NO}_{\mathrm{x}}$ Emissions Response to Aromatics Content $\ldots \ldots \ldots \ldots \ldots \ldots \ldots \ldots \ldots \ldots \ldots \ldots$

$4.4 \mathrm{NO}_{\mathrm{x}}$ Emissions Response to Sulfur Content $\ldots \ldots \ldots \ldots \ldots \ldots \ldots \ldots \ldots \ldots \ldots \ldots \ldots \ldots$

$4.5 \quad \mathrm{NO}_{\mathrm{x}}$ Emissions Response to Oxygen Content $\ldots \ldots \ldots \ldots \ldots \ldots \ldots \ldots \ldots \ldots \ldots \ldots$ II-19

4.6 PM Emissions Response to Cetane Effects $\ldots \ldots \ldots \ldots \ldots \ldots \ldots \ldots \ldots \ldots \ldots \ldots \ldots$ II-23

4.7 PM Emissions Response to Natural and Additized Cetane $\ldots \ldots \ldots \ldots \ldots \ldots \ldots \ldots \ldots$ II-24

$4.8 \quad$ PM Emissions Response to Cetane Improvement $\ldots \ldots \ldots \ldots \ldots \ldots \ldots \ldots \ldots \ldots \ldots \ldots$ II-24

4.9 PM Emissions Response to Sulfur and Aromatics Content $\ldots \ldots \ldots \ldots \ldots \ldots \ldots \ldots \ldots$ II-25

4.10 PM Emissions Response to Sulfur Content and Specific Gravity $\ldots \ldots \ldots \ldots \ldots \ldots \ldots$ II-25

4.11 PM Emissions Response to Sulfur Content and Cetane Improvement $\ldots \ldots \ldots \ldots \ldots \ldots$ II-26 



\title{
ACRONYMS AND ABBREVIATIONS
}

\author{
AEO Annual Energy Outlook \\ API American Petroleum Institute \\ ASTM American Society for Testing and Materials \\ bbl Barrel \\ BGY Billion gallons per year \\ CARB California Air Resources Board \\ CG Conventional gasoline \\ cpg Cents per gallon \\ cSt Centistokes \\ DeS Desulfurization \\ DOE Department of Energy \\ EGR Exhaust gas recirculation \\ EIA Energy Information Administration \\ EP End point \\ EPA Environmental Protection Agency \\ EtOH Ethanol \\ E200 The cumulative volume percent evaporated at $200^{\circ} \mathrm{F}$ in ASTM test \\ D86-87: Distillation of Petroleum Products \\ E300 The cumulative volume percent evaporated at $300^{\circ} \mathrm{F}$ in ASTM test \\ D86-87: Distillation of Petroleum Products \\ $\mathrm{F}$ \\ Fahrenheit \\ FBP Final boiling point \\ FCC Fluid catalytic cracking \\ FOE Fuel oil equivalent \\ IBP Initial boiling point
}




\begin{tabular}{|c|c|}
\hline LL & Lower limit \\
\hline LPG & Liquefied petroleum gas \\
\hline LSD & Low sulfur diesel \\
\hline LSG & Low sulfur gasoline \\
\hline M & Motor octane number \\
\hline $\max$ & Maximum \\
\hline MBCD & Thousand barrels per calendar day \\
\hline MBD & Thousand barrels per day \\
\hline MBPD & Thousand barrels per day \\
\hline MBSD & Thousand barrels per stream day \\
\hline $\mathrm{mg} / \mathrm{mi}$ & Milligrams per mile \\
\hline $\min$ & Minimum \\
\hline MM & Million \\
\hline mpg & Miles per gallon \\
\hline MST/D & Thousand short tons per day \\
\hline MTBE & Methyl tertiary butyl ether \\
\hline NOx & Oxides of nitrogen \\
\hline NPC & National Petroleum Council \\
\hline NPRA & $\begin{array}{l}\text { National Petroleum Refiners Association or National Petrochemical \& } \\
\text { Refiners Association }\end{array}$ \\
\hline ORNL & Oak Ridge National Laboratory \\
\hline PADD & Petroleum Administration for Defense District \\
\hline PM & Particulate matter \\
\hline ppm & Part per million \\
\hline psi & Pounds per square inch \\
\hline $\mathrm{R}$ & Research octane number \\
\hline
\end{tabular}




$\begin{array}{ll}\text { RFD } & \text { Reformulated diesel fuel } \\ \text { RFG } & \text { Reformulated gasoline } \\ \text { ROI } & \text { Return on investment } \\ \text { RVP } & \text { Reid vapor pressure } \\ \text { RYM }{ }_{2002} & \text { Refinery Yield Model, updated in 2002 } \\ \text { ST/CD } & \text { Short tons per calendar day } \\ \text { TAP } & \text { Toxic air pollutant } \\ \text { Txx } & \text { The temperature at which } \mathrm{xx}(0<\mathrm{xx}<100) \text { percent of a test volume of } \\ & \text { fuel is evaporated in ASTM test D86-87: Distillation of Petroleum } \\ \text { UL } & \text { Products } \\ \text { ULSD } & \text { Upper limit } \\ \text { UrL } & \text { Ultra low sulfur diesel } \\ \text { VOC } & \text { Volatile organic compound } \\ \text { vol } & \text { Volume } \\ \text { wt } & \text { Weight } \\ \text { WTI } & \text { West Texas Intermediate }\end{array}$





\begin{abstract}
The Oak Ridge National Laboratory Refinery Yield Model has been used to study the refining cost, investment, and operating impacts of specifications for reformulated diesel fuel (RFD) produced in refineries of the U.S. Midwest in summer of year 2010. The study evaluates different diesel fuel reformulation investment pathways. The study also determines whether there are refinery economic benefits for producing an emissions reduction RFD (with flexibility for individual property values) compared to a vehicle performance RFD (with inflexible recipe values for individual properties). Results show that refining costs are lower with early notice of requirements for RFD. While advanced desulfurization technologies (with low hydrogen consumption and little effect on cetane quality and aromatics content) reduce the cost of ultra low sulfur diesel fuel, these technologies contribute to the increased costs of a delayed notice investment pathway compared to an early notice investment pathway for diesel fuel reformulation. With challenging RFD specifications, there is little refining benefit from producing emissions reduction RFD compared to vehicle performance RFD. As specifications become tighter, processing becomes more difficult, blendstock choices become more limited, and refinery benefits vanish for emissions reduction relative to vehicle performance specifications. Conversely, the emissions reduction specifications show increasing refinery benefits over vehicle performance specifications as specifications are relaxed, and alternative processing routes and blendstocks become available. In sensitivity cases, the refinery model is also used to examine the impact of RFD specifications on the economics of using Canadian synthetic crude oil. There is a sizeable increase in synthetic crude demand as ultra low sulfur diesel fuel displaces low sulfur diesel fuel, but this demand increase would be reversed by requirements for diesel fuel reformulation.
\end{abstract}





\section{EXECUTIVE SUMMARY}

\section{ES.1 OBJECTIVES AND KEY FINDINGS}

The Oak Ridge National Laboratory Refinery Yield Model (ORNL-RYM $\mathrm{M}_{2002}$ ), updated in 2002, has been used to study the refining cost, investment, operating, and crude oil impacts of specifications for reformulated diesel fuel (RFD) in case studies required by the U.S. Department of Energy (DOE) Offices of Policy and International Affairs, Energy Efficiency and Renewable Energy, and Fossil Energy. Key findings of this study include the following:

- $\quad$ Refining costs are lower with early notice of product quality requirements for RFD. With early notice of RFD requirements, refinery capital investment has not yet been made to satisfy the ultra low sulfur diesel fuel (ULSD) requirement of year 2006, and there is greater flexibility for investment planning. With delayed notice of requirements, investment flexibility is less because capital investment has been sunk to satisfy the ULSD sulfur requirement.

- While advanced desulfurization technologies reduce the cost of ULSD, these technologies can contribute to the increased costs of a delayed notice compared to an early notice investment pathway to RFD. With relatively small impact on cetane and aromatics quality, these technologies are on a suboptimal pathway for RFD production.

- There is little refining economic benefit from using flexible emissions reduction specifications rather than vehicle performance recipe specifications, when recipe specifications are challenging. This is because processing options and blendstocks are very limited for achieving challenging recipe specifications.

- Emissions reduction specifications show increasing refinery benefits over vehicle performance recipe specifications, as those recipe specifications are relaxed, and alternative processing routes and blendstocks become available.

- There is a sizeable increase in synthetic crude oil demand as ULSD displaces low sulfur diesel fuel, but this demand increase would be reversed by a requirement for diesel fuel reformulation.

\section{ES.2 PREMISES AND CASES}

Table ES-1 shows on-road No. 2 diesel fuel regulations and timing for several regulatory authorities and for the World Wide Fuel Charter. Given that the diesel fuel requirements for California and Texas will be largely satisfied by alternative performance-based specifications, the most challenging requirements for onroad diesel fuel are those specifications recommended by the European Union and the World Wide Fuel Charter, particularly specifications for cetane number and total aromatics. Global vehicle and engine manufacturing associations support the World Wide Fuel Charter on the basis that "Consistent fuel quality world-wide is necessary to market high-quality automotive products matching world-wide customer performance and environmental needs."

The examination of challenging RFD specifications and their impacts on refinery investment requirements and operating costs inevitably raises a wide range of policy issues. In establishing specific premises for this study, we must make assumptions about the resolution of a number of currently unresolved issues. One should not interpret these premises as ORNL or DOE views regarding the appropriate resolution of the 
underlying policy issues, but only as one possible set of reasonable assumptions for doing the analysis. Key premises, which are policy-related in an important way, include the following:

(1) We are examining a national on-road diesel fuel reformulation requirement because there is significant interest, among various parties, in changing diesel fuel quality (beyond sulfur reductions) to enable either lower in-use emissions, new vehicle emission control system performance, or vehicle operating characteristics. Understanding how the timing and specific nature of such a requirement interacts with other requirements, refinery operation or emerging crude oil options is important to policy formulation.

(2) The interaction of an RFD requirement with the existing 2006 ULSD requirement will be significant in terms of investment strategics that refiners might pursue. While we have assumed a 2010 start date for an RFD requirement, it could happen sooner or later than that, and refiners might invest for ULSD in 2006 with the forward knowledge of an RFD requirement, or they might not. This analysis examines cases covering both ends of the range of possibilities. Refiners invest once for both programs or they invest sequentially, first only for ULSD and then for RFD.

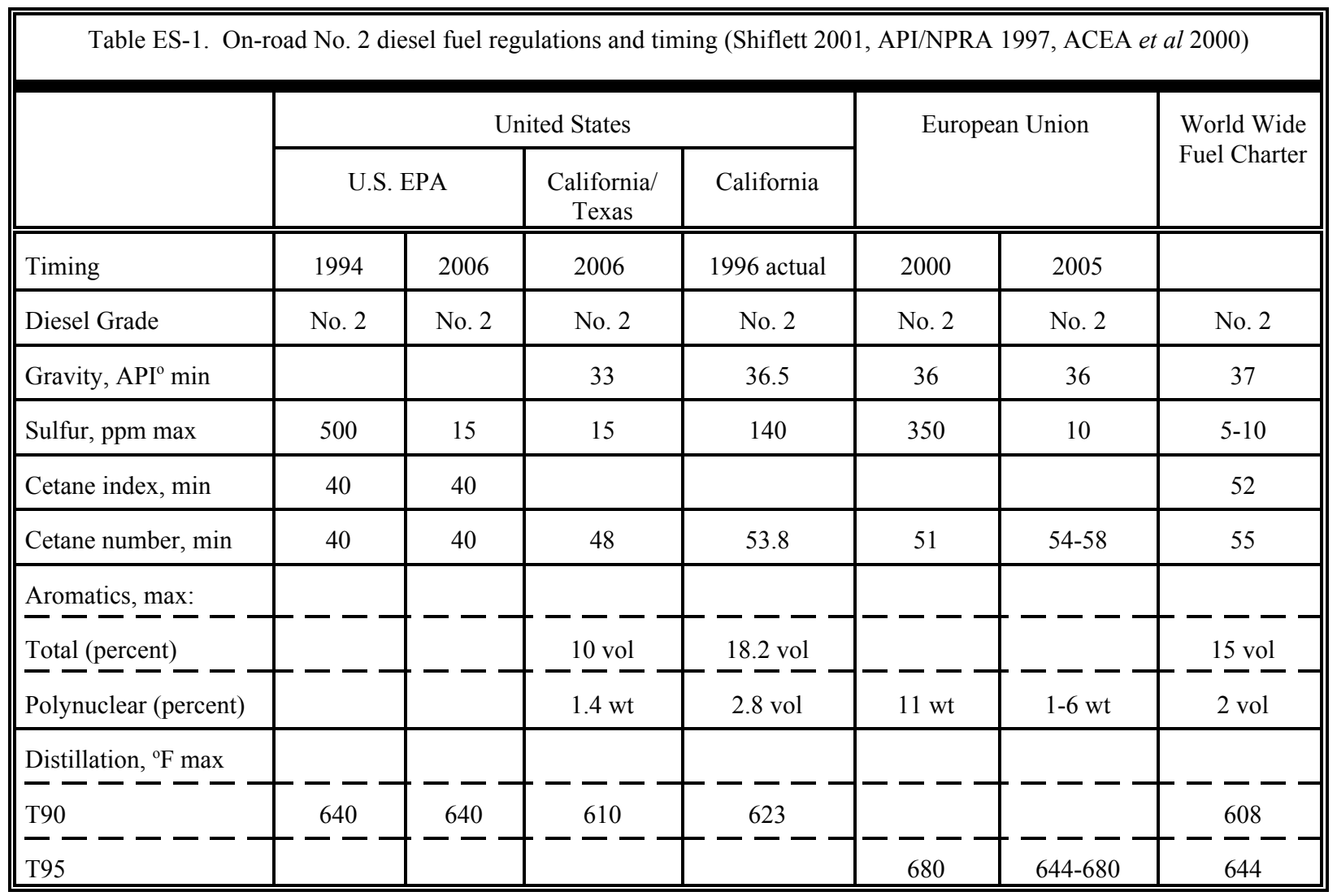

(3) RFD could be defined in terms of its emission performance or in terms of specific levels for fuel properties such as cetane number or aromatics content. Both paths have been used in various regulatory regimes world-wide. Because the automobile industry's World Wide Fuel Charter advocates the specific parameter approach, we call this RFD a "vehicle performance fuel." Alternatively, states like California have allowed equivalent emissions performance fuels, and we have designated such an RFD in this analysis as an "emissions reduction fuel." While 
emissions performance will be set equal, vehicle operational impacts and refining cost impacts could be different for vehicle performance RFD compared to emissions reduction RFD. We analyze both RFDs in terms of refinery impacts.

(4) Changes to off-road diesel fuel quality are quite likely in the time frame of this analysis, but the specifics are not known. ULSD (15 ppm maximum sulfur content) may become the needed fuel for some portion of the off-road market while some low sulfur diesel fuel (500 ppm maximum sulfur content) may remain in the diesel fuel pool due to phase-in of the ULSD requirement. For this analysis, a volume of diesel equal to off-road volume is assumed to be at $500 \mathrm{ppm}$ and the rest at $15 \mathrm{ppm}$ (i.e., ULSD), but the actual end use markets for the two sulfur levels may be more mixed than that. The critical implied assumption is that all high sulfur (greater than $500 \mathrm{ppm}$ ) diesel fuel disappears from the refinery slate by 2010 .

(5) Changes to gasoline requirements are likely in the time frame relevant to this analysis. We have premised the requirements contained in current proposed legislation (U.S. Senate Bill S.517, subsequently amended into U.S. House of Representatives Bill H.R.4) as a plausible set of new requirements. Despite significant changes in that legislation (e.g., MTBE ban, elimination of the reformulated gasoline oxygenate requirement and mandated ethanol use), the actual impact on refinery operations in our study region, the U.S. Midwest, is relatively small because of preexisting ethanol use and the almost total absence of MTBE use in that region, and the limited interactions between gasoline quality and diesel fuel reformulation.

(6) Finally, we examine the interaction between greater use of Canadian synthetic crude oils and these tighter diesel fuel quality specifications, with the implicit assumptions that there will be a significant growth in the volume of that synthetic crude production and that U.S. Midwestern refineries are a natural market for that crude. The need for effective integration of refinery changes in the U.S. (driven by changing product quality requirements) and expanded synthetic crude production in Canada is a possible use of this part of the analysis.

Based on these premises, the study case design is summarized in Table ES-2. For each of the two different reformulation pathways (vehicle performance and emissions reduction), two different investment pathways are investigated in the study cases, in order to understand the refinery benefits of early notice of requirements for more stringent specifications. With early notice of RFD requirements, refinery capital investment would not yet be made to satisfy the ULSD sulfur requirement of year 2006, giving greater flexibility for investment planning. With delayed notice of requirements, capital investment would have been sunk to satisfy the ULSD sulfur requirement, resulting is less investment flexibility. 


\begin{tabular}{||c|l||}
\hline \multicolumn{2}{||c||}{ Table ES-2. Diesel fuel reformulation case studies for U.S. Midwestern refineries in } \\
summer 2010
\end{tabular}

\section{ES.3 EMISSIONS MODELING}

In the study cases, cetane number and aromatics content are specified for the vehicle performance RFD. The emissions quality of the resulting vehicle performance RFD is then used to specify requirements for emissions reduction RFD. Unfortunately, it is very difficult to accurately estimate the change in diesel emissions in terms of changes in individual fuel properties. This difficulty is due to the interdependence, or correlation, of certain fuel properties, and to differences in the way fuel property changes can be achieved in refinery processing and blending. For example, sulfur reduction may or may not be accompanied by significant aromatics reduction, depending on the processing route (e.g., hydrocracking versus desulfurization without dearomatization).

Given these difficulties, emissions benefits may be mis-estimated for a policy based on analysis of individual property effects. Furthermore, unnecessary refining costs may be incurred, with possible impacts on fuel supply. Our methodology solves the problems of individual property correlations by estimating emissions in terms of independent vector groupings of the individual properties. These vector groupings are the eigenvectors derived by Principal Components Analysis. Each eigenvector represents a unique and mathematically independent characteristic of diesel fuel. Because of their relationships with refinery processing and blending, these eigenvectors are called eigenfuels in our work. As demonstrated in past work, eigenfuels have many advantages, including: 
Simplification of the analysis, because the mathematical independence of eigenfuels eliminates correlations among the variables and the associated complications.

Economy of representation, because a smaller number of such vector variables may effectively replace a larger number of original variables.

Greater understanding of the patterns of variation that are important to emissions, and how these patterns relate to refinery processing and blending.

New insight into the optimal economic formulation of fuels to reduce emissions.

Given limitations of the available diesel engine test data, we find that the eigenfuel method leads to new perspectives on diesel fuel-emissions relationships:

Fuel properties are only surrogate variables for underlying causal factors. Much of the emissions reduction seen in past testing comes from reducing highly aromatic cracked stocks in diesel fuel. Because these stocks are low in cetane number and high in density, researchers have tended to attribute the emissions reductions to the increase in cetane number or reduction in density associated with their removal, rather than to the compositional change itself.

How one varies a fuel property can be the most important factor in determining the emissions response. A given fuel property can be changed in many ways, and a unit change in that property can produce markedly different effects on emissions depending on how that change is introduced.

\section{ES.4 RFD STUDY FINDINGS}

With its eigenfuel-based representation of emissions, ORNL-RYM ${ }_{2002}$ produced the RFD study findings summarized in Table ES-3. The table shows that refining costs are lower with early notice (parallel investment) of product quality requirements for on-road diesel fuel. While advanced desulfurization technologies (with low hydrogen consumption and little effect on cetane quality and aromatics content) reduce the cost of ULSD, these technologies contribute to the increased costs of a sequential investment pathway compared to a parallel investment pathway to RFD. In the sequential investment pathway, advanced desulfurization unit utilization and the associated cash margin fall. The sequential investments include substantial conversion of second-stage hydrodesulfurization capacity to second stage dearomatization capacity. 


\begin{tabular}{|c|c|c|c|}
\hline \multicolumn{4}{|c|}{ Table ES-3. Diesel fuel reformulation study findings } \\
\hline Case & $\begin{array}{c}\text { On-road diesel } \\
\text { upgrading pathway } \\
\end{array}$ & $\begin{array}{c}\text { Cost } \\
\text { increase }^{\mathrm{a}} \\
\end{array}$ & Comment \\
\hline Base Case 1 & $\begin{array}{l}\text {-Low sulfur } \\
\text {-Investment as required }\end{array}$ & 0.0 & \\
\hline Case 1.1 & $\begin{array}{l}\cdot \text { ULSD } \\
\cdot \text { Reformulated for vehicle } \\
\text { performance } \\
\text { •Early notice of } \\
\text { requirements } \\
\cdot \text { Parallel investment }\end{array}$ & 9.0 & $\begin{array}{l}\text { Due to high cetane specification, distillate upgrading is via } \\
\text { 2-stage deep hydroprocessing, not advanced desulfurization } \\
\text { technologies that selectively remove sulfur without cetane } \\
\text { improvement. }\end{array}$ \\
\hline Case 1.2 & $\begin{array}{l}\cdot \text { ULSD } \\
\cdot \text { Reformulated for } \\
\text { emissions reduction } \\
\text {-Early notice of } \\
\text { requirements } \\
\text {-Parallel investment }\end{array}$ & 8.9 & $\begin{array}{l}\text { Emissions targets are based on Case } 1.1 \text { (relative to reference } \\
\text { commercial fuel, NOx reduction } \geq 8.3 \text { percent and PM } \\
\text { reduction } \geq 19.8 \text { percent). With little blendstock flexibility } \\
\text { (virtually same stocks selected as in Case } 1.1 \text { ), emissions } \\
\text { reduction specifications have little refining benefit, if the } \\
\text { objective is RFD emissions quality improvement. }\end{array}$ \\
\hline Base Case 2 & $\begin{array}{l}\text {-ULSD } \\
\text {-Sequential investment } \\
\text { (step 1) } \\
\text {-Some newer technologies }\end{array}$ & 5.2 & $\begin{array}{l}\text { Investment in advanced desulfurization technology results in } \\
\text { lower costs. Sensitivity runs show cost increase of } 7.9 \mathrm{cpg} \\
\text { via 2-stage deep hydroprocessing, with no investment } \\
\text { allowed in advanced desulfurization; and cost increase of } 3.7 \\
\text { cpg with no constraints on investment in advanced } \\
\text { desulfurization. }\end{array}$ \\
\hline Case 2.1 & $\begin{array}{l}\text {-ULSD } \\
\text { peformulated for vehicle } \\
\text { performance } \\
\text {-Delayed notice of } \\
\text { requirements } \\
\text {-Sequential investment } \\
\text { (step 2) }\end{array}$ & 9.7 & $\begin{array}{l}\text { In second step to high cetane/low aromatics, there is drop in } \\
\text { utilization of advanced desulfurization, and refineries with } \\
\text { this capacity must purchase high cetane/low aromatics } \\
\text { stocks from those refineries with } 2 \text {-stage deep } \\
\text { hydroprocessing and/or invest in distillate dearomatization } \\
\text { capacity. Capital investment increases, and cost increases } \\
0.7 \text { cpg compared to early notice pathway of Case } 1.1 \text {. }\end{array}$ \\
\hline Case 2.2 & $\begin{array}{l}\text {-ULSD } \\
\text { - Reformulated for } \\
\text { emissions reduction } \\
\text {-Delayed notice of } \\
\text { requirements } \\
\text {-Sequential investment } \\
\text { (step 2) }\end{array}$ & 9.7 & $\begin{array}{l}\text { Emissions targets are based on Case } 2.1 \text { (relative to reference } \\
\text { commercial fuel, NOx reduction } \geq 8.2 \text { percent and PM } \\
\text { reduction } \geq 19.8 \text { percent). There are no refinery benefits } \\
\text { from using emissions reduction versus vehicle performance } \\
\text { specifications in these cases with very challenging } \\
\text { specifications. As specifications become tighter, refinery } \\
\text { benefits vanish for the emissions reduction versus vehicle } \\
\text { performance specifications. Conversely, emissions } \\
\text { reductions specifications show increasing refinery benefits } \\
\text { over recipe specifications as those recipe specifications are } \\
\text { relaxed. }\end{array}$ \\
\hline $\begin{array}{l}\text { Synthetic } \\
\text { Crude } \\
\text { Sensitivities }\end{array}$ & $\begin{array}{l}\text { There is a sizeable increase } \\
\text { diesel falls from } 500 \mathrm{ppm} \text { to } \\
\text { reformulation. }\end{array}$ & $\begin{array}{l}\text { nthetic cr } \\
\text { pm, but } t\end{array}$ & $\begin{array}{l}\text { demand when the maximum sulfur specification for on-road } \\
\text { demand increase would be reversed by diesel fuel }\end{array}$ \\
\hline
\end{tabular}


With the objective of RFD emissions quality improvement, there is little refining economic benefit from using the flexible emissions reduction specifications rather than the vehicle performance recipe specifications in these cases, which have very challenging specifications. As specifications become tighter, processing becomes more difficult, blendstock choices become more limited, and refinery benefits vanish for emissions reduction relative to vehicle performance specifications.

Conversely, emissions reduction specifications show increasing refinery benefits over vehicle performance recipe specifications as those recipe specifications are relaxed, and alternative processing routes and blendstocks become available. For example, using average specifications rather than cap specifications in our study reduces investment and RFD production costs for emissions reduction compared to vehicle performance.

In sensitivity cases, ORNL-RYM 2002 is used to examine the impact of RFD specifications on the economics of using Canadian synthetic crude oil. There is a sizeable increase in synthetic crude demand as ULSD fuel displaces low sulfur diesel fuel, but this demand increase would be reversed by a requirement for diesel fuel reformulation. 



\section{PART I. THE REFINERY STUDY}

\section{I-1. INTRODUCTION}

The Oak Ridge National Laboratory Refinery Yield Model (ORNL-RYM ${ }_{2002}$ ), updated in 2002, has been used to study the refining cost, investment, operational, and crude oil impacts of specifications for reformulated diesel fuel (RFD) in case studies required by the U.S. Department of Energy (DOE) Offices of Policy and International Affairs, Energy Efficiency and Renewable Energy, and Fossil Energy.

Table 1.1 shows No. 2 on-road diesel fuel regulations and timing for several regulatory authorities and for the World Wide Fuel Charter. Given that the diesel fuel requirements for California and Texas will be largely satisfied by alternative performance-based specifications, the most challenging requirements for onroad diesel fuel are those specifications recommended by the European Union and the World Wide Fuel Charter, particularly specifications for cetane number and total aromatics.

Global vehicle and engine manufacturing associations support the World Wide Fuel Charter on the basis that "Consistent fuel quality world-wide is necessary to market high-quality automotive products matching worldwide customer performance and environmental needs." In its "Technical Background for Harmonised Fuel Recommendations," the World Wide Fuel Charter discusses its views of the emissions reduction potential of changes in individual diesel fuel properties. In fact, it is very difficult to accurately estimate the change in diesel emissions in terms of changes in individual fuel properties. This difficulty is due to the interdependence, or correlation, of certain fuel properties, and to differences in the way fuel property changes can be achieved in refinery processing and blending. For example, sulfur reduction may or may not be accompanied by significant aromatics reduction, depending on the processing route (e.g., hydrocracking versus desulfurization without dearomatization).

Given these difficulties, emissions benefits may be mis-estimated for a regulation based on analysis of individual property effects. Furthermore, unnecessary refining costs may be incurred, with possible impacts on fuel supply. Our eigfenfuel methodology, described in PART II of this report, solves the problems of individual property correlations by estimating emissions in terms of independent vector variables. Eigenfuels, which are related to underlying refinery and blending processes, provide an unambiguous method for costeffective blending of refinery stocks. 
Table 1.1. On-road No. 2 diesel fuel regulations and timing (Shiflett 2001, API/NPRA 1997, ACEA et al 2000)

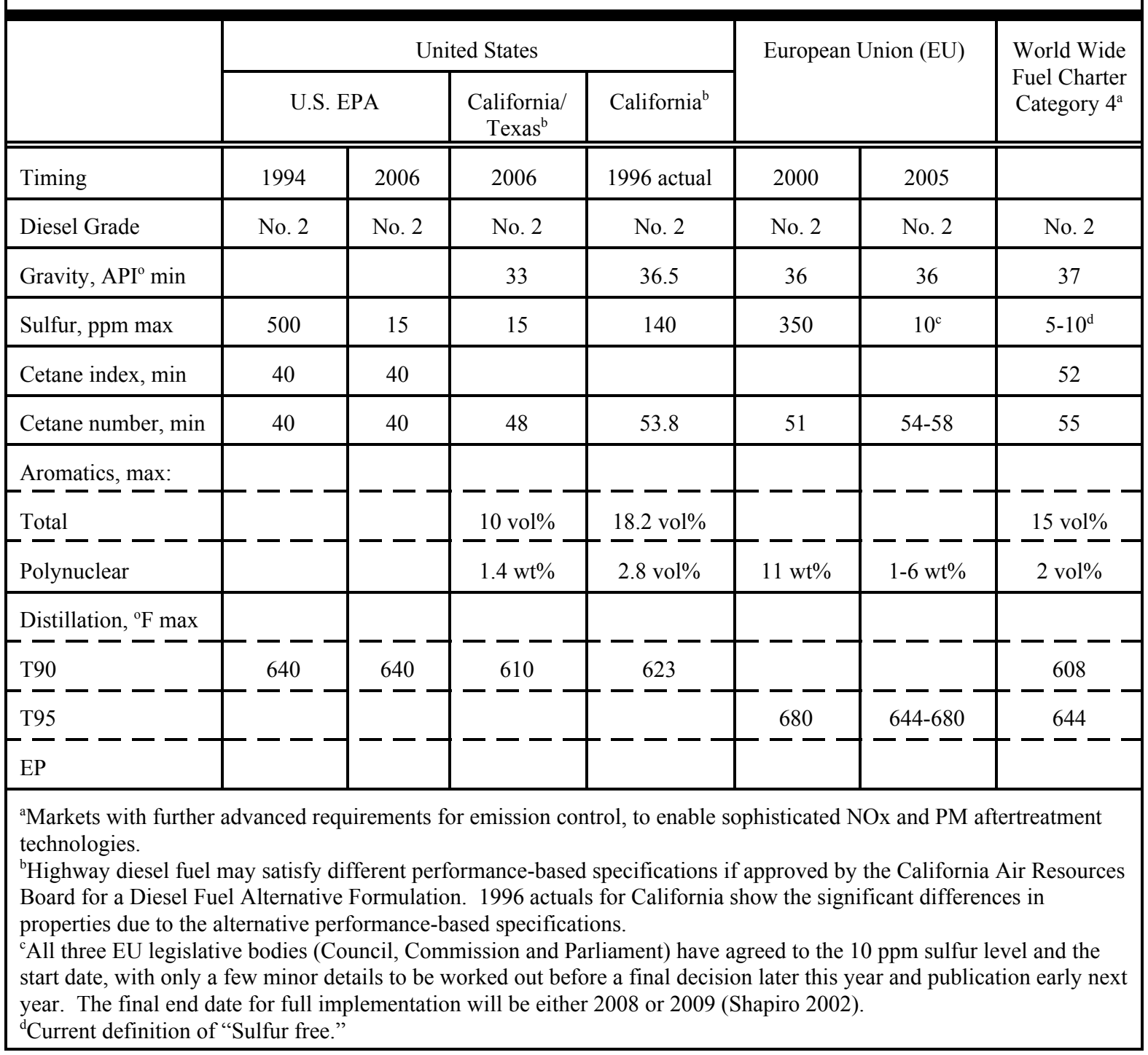

With its eigenfuel-based emissions representations, ORNL-RYM $\mathrm{M}_{202}$ has been used to estimate the refining cost and investment impacts of two different diesel fuel reformulation pathways. The reformulation pathways show whether there are refinery economic benefits for emissions reduction specifications (with flexibility for individual fuel properties) compared to vehicle performance specifications (with an inflexible recipe for individual fuel properties), given equal emissions performance for the two RFD requirements. For each of the two different reformulation pathways, two different investment pathways have been investigated, in order to understand the refinery economic benefits of early notice of requirements for more stringent specifications. With early notice of RFD requirements, refinery capital investment would not yet be made to satisfy the ultra low sulfur diesel (ULSD) fuel sulfur requirement of year 2006, giving greater flexibility for investment planning. With delayed notice of requirements, capital investment has been sunk to satisfy the ULSD sulfur requirement, resulting in less flexibility.

In sensitivity cases, ORNL-RYM ${ }_{2002}$ has also been used to examine the impact of RFD specifications on the economics of using Canadian synthetic crude oil. As the production of synthetic crude oil increases, with economic displacement of conventional crude oils for refining, oilsands operators and refiners will be 
technically challenged by requirements to improve diesel fuel cetane number, jet fuel smoke point, and heavy gas oil quality for fluid catalytic cracking feed (Yui 2000; Yui and Chung 2001). 



\section{I-2. THE ORNL REFINERY YIELD MODEL}

\section{I-2.1 ORNL-RYM 2002}

ORNL-RYM $\mathrm{M}_{2002}$ is a linear program representing over 75 refining processes which can be used to produce up to 50 different products from more than 180 crude oils. An investment module provides for the addition of processing capacity (DOE 1984a,b; Tallett and Dunbar 1988; Tallett, Dunbar and Leather 1992). ORNL-RYM ${ }_{2002}$ tracks gravity, cetane index, aromatics, sulfur, flash point, pour point, viscosity, hydrogen content, heat of combustion, distillation temperatures, and pollutant emissions on all diesel component streams. In separate data tables in ORNL-RYM ${ }_{2002}$, diesel blending components are identified; blending values are assigned to these components; and specifications are set for diesel products. ORNL-RYM ${ }_{2002}$ incorporates diesel fuel blending using the vector-based eigenfuel concept to satisfy emissions specifications for oxides of nitrogen (NOx) and particulate matter (PM) (McAdams, Crawford, and Hadder 2002; Crawford 2002).

Properties for other distillates and for gasolines are handled in a similar fashion. ORNL-RYM ${ }_{2002}$ incorporates gasoline blending to satisfy formula and emissions standards mandated by the Clean Air Act Amendments of 1990 and described by the EPA Complex Model, which predicts gasoline pollutant emissions in terms of gasoline properties (Korotney 1993). ORNL-RYM ${ }_{2002}$ also represents requirements of the toxics anti-backsliding rulemaking of 2001 (EPA 2001).

Overoptimization can occur as a result of the ORNL-RYM ${ }_{2002}$ use of a modeling concept in which refinery streams with identical distillation cut points are kept separate through different refining processes. Ratio constraints on refinery streams can be used to avoid unrealistic separation of streams with identical distillation cut points. With ratio constraints, the proportions of streams entering a process are constrained to equal the proportions of those streams produced at a source process. This study makes use of ratio constraints in gasoline production, based on calibration results. Ratio constraints are also used for distillate deep desulfurization and dearomatization processes.

It is important to recognize that refineries within a region can vary widely in technical capability, and that refineries are subject to temporal variations in complex operations. A refining outcome (e.g., investment cost) can span a range, and this range has uncertainty.

\section{I-2.2 MODEL UPDATE}

Specifically for this study, ORNL-RYM 2002 was updated to include:

The most recent process configuration and revamp cost information for distillate desulfurization units.

Representations of advanced desulfurization technologies that selectively remove sulfur, with low hydrogen consumption.

Representation of the most recent catalyst developments.

Representation of revamping of existing steam reforming hydrogen production units to increase capacity and reduce energy consumption and operating costs.

Representation of a debottlenecking feature to enable capacity addition on selected units at a fraction of new unit costs. 
Representation of process unit capital cost trending over time to reflect the potential benefits of later process unit additions.

A number of other technology and features enhancements, discussed in Appendices I-A and I-B (Dunbar and Tallett 2002a,b). 


\section{I-3. PREMISES}

\section{I-3.1 POLICY ISSUES}

The examination of the impacts of an RFD requirement on refinery investment and operating costs inevitably raises a wide range of policy issues. These issues have to be addressed in order to establish the framework for the subject. In establishing specific premises for this study, we must make assumptions about the resolution of a number of currently unresolved issues. One should not interpret these premises as ORNL or DOE views as to the appropriate resolution of the underlying policy issues, but only as one possible set of reasonable assumptions for doing the analysis. We have highlighted below some of the key premises that are policy-related in an important way:

(1) We are examining a national on-road diesel fuel reformulation requirement because there is significant interest, among various parties, in changing diesel fuel quality (beyond sulfur reductions) to enable either lower in-use emissions, new vehicle emission control system performance, or vehicle operating characteristics (e.g., cold startability, noise, idle quality). Understanding how the timing and specific nature of such a requirement interacts with other requirements, refinery operation or emerging crude oil options is important to policy formulation.

(2) The interaction of such an RFD requirement with the existing 2006 ULSD requirement will be significant in terms of investment strategics that refiners might pursue. While we have assumed a 2010 start date for an RFD requirement, it could happen sooner or later than that, and refiners might invest for ULSD in 2006 with the forward knowledge of an RFD requirement, or they might not. This analysis examines cases covering both ends of the range of possibilities. Refiners invest once for both programs (effectively) in 2006 or they invest sequentially, first only for ULSD and then for RFD.

(3) RFD could be defined in terms of its emission performance or in terms of specific levels for fuel properties such as cetane number or aromatics content. Both paths have been used in various regulatory regimes world-wide. Because the auto industry's World Wide Fuel Charter advocates the specific parameter approach, we call this RFD a "vehicle performance fuel." Alternatively, states like California have allowed equivalent emissions reduction fuels, and we have specified such an RFD in this analysis as an "emissions reduction fuel." While emissions performance will be set equal, vehicle operational impacts and refining cost impacts could be different for vehicle performance RFD compared to emissions reduction RFD. We analyze both RFDs in terms of refinery impacts.

(4) Changes to off-road diesel fuel quality are quite likely in the time frame of this analysis, but the specifics are not known. ULSD (at 15 ppm maximum sulfur) may become the needed fuel for some portion of the off-road market while some low sulfur diesel fuel (at $500 \mathrm{ppm}$ maximum sulfur) may remain in the diesel fuel pool due to the phase-in of the ULSD requirement. For this analysis, a volume of diesel equal to off-road volume is assumed to be at $500 \mathrm{ppm}$ and the rest at $15 \mathrm{ppm}$ (i.e., ULSD), but the actual end use markets for the two sulfur levels may be more mixed than that. The critical implied assumption is that all high sulfur (greater than $500 \mathrm{ppm}$ ) diesel fuel disappears from the PADD II (Petroleum Administration for Defense District II, the U.S. Midwest) refinery slate by 2010.

(5) Changes to gasoline requirements are likely in the time frame relevant to this analysis. We have premised the requirements contained in current proposed legislation (U.S. Senate Bill S.517, subsequently amended into U.S. House of Representatives Bill H.R.4) as a plausible set of new 
requirements. Despite the significant changes in that legislation (MTBE ban, elimination of the reformulated gasoline [RFG] oxygenate requirement and mandated ethanol use), the actual impact on PADD II refinery operations for the purposes of our analysis is relatively small because of pre-existing ethanol use and the almost total absence of MTBE use in that region, and the limited interactions between gasoline quality and diesel fuel reformulation.

(6) Finally, we examine the interaction between greater use of Canadian synthetic crude oils and these tighter diesel fuel quality specifications, with the implicit assumption that there will be a significant growth in the volume of that synthetic crude production and that PADD II refineries are a natural market for that crude. The need for effective integration of refinery changes in the U.S. (driven by changing product quality requirements) and expanded synthetic crude production in Canada is a possible use of this part of the analysis.

\section{I-3.2 STUDY PERIOD AND GEOGRAPHIC AREA}

The study period is summer of year 2010. The study area is PADD II, a 15 state area in the U.S. Midwest with 27 operable refineries, annually producing 22 percent of all gasoline and 25 percent of all on-road diesel fuel produced in the U.S. With the exception of PADD IV (the U.S. Rocky Mountain area), PADD II has the highest percentage production of on-road diesel fuel (relative to refinery net production of finished petroleum products). Also with the exception of PADD IV, PADD II has the highest percentage of imports of Canadian crude oil (relative to total refinery input of crude oil), which is a consideration for the sensitivity case study of the impact of RFD specifications on the economics of using Canadian synthetic crude oil. PADD IV was not selected because of its relatively small refining capacity and low complexity.

Of the 27 PADD II refineries, 13 have distillate hydrotreating, and we assume that these refineries are currently producers of low sulfur diesel fuel. Different studies have focused on a range of possibilities for refinery participation in on-road diesel fuel production for 2006-2007. Baker \& O'Brien (2001) believes that only current low sulfur diesel fuel producers are likely to continue in the ULSD market. On the other hand, MathPro (2002) concludes that some refineries that do not now produce low sulfur diesel fuel may have incentives to produce ULSD. For our more distant outlook of year 2010 (with a 24 percent increase in onroad diesel fuel production, compared to year 2000), we assume that ULSD/RFD production can be spread among all refineries in PADD II.

\section{I-3.3 TECHNICAL PREMISES}

Technical premises for product slates and revenues, raw materials and costs, and process capacity data as loaded in ORNL-RYM $\mathrm{R}_{2002}$ - are shown in Table 3.1 and Table 3.2. These modeling data are based on information sources discussed in the following paragraphs. 


\begin{tabular}{|c|c|c|c|c|c|}
\hline \multicolumn{3}{|c|}{ Raw materials } & \multicolumn{3}{|l|}{ Products } \\
\hline CRUDE/RAW MATERIAL & \$/BARREL & $\mathrm{MBD}$ & PRODUCT & \$/BARREL & MBD \\
\hline COMPOSITE CRUDE & 24.99 & $<10000$, WITH & PROCESS GAS C2-FOE & 23.22 & $\geq 0$ \\
\hline \multicolumn{3}{|c|}{ COMPOSITION CORRESPONDING TO FOLLOWING MIX: } & $\begin{array}{l}\text { STILL GAS TO PETROCHEM } \\
\text { ETHANE }\end{array}$ & 23.22 & $\begin{array}{l}\geq 0 \\
\geq 0\end{array}$ \\
\hline ALGERIAN SAHARAN & 28.97 & 21.18 & ETHYLENE & 26.81 & $\geq 0$ \\
\hline ALASKA COOK INLET & 25.09 & 11.48 & PROPYLENE TO PETROCHEM & 28.61 & $\geq 0$ \\
\hline ALASKA PRUDHOE BAY & 23.89 & 7.95 & PROPANE FUEL (LPG) & 32.32 & $\geq 0$ \\
\hline CALIF SARDO & 18.12 & 26.56 & NORMAL BUTANE & 25.86 & $\leq 32.27$ \\
\hline $\begin{array}{l}\text { CALIF VENTURA } \\
\text { CHINESE EXPORT }\end{array}$ & $\begin{array}{l}25.56 \\
23.44\end{array}$ & $\begin{array}{l}6.389 \\
4.819\end{array}$ & N BUTYLENE & 31.61 & $\geq 0$ \\
\hline CANADIAN CONDENSATE & 35.17 & 138.4 & ISO BUTANE & 30.41 & $\geq 0$ \\
\hline CANADIAN RGLD & 27.28 & 380.5 & ISO BUTYLENE & 31.61 & $\geq 0$ \\
\hline GABON GAMBA & 23.88 & 11.48 & *EtOH use based on "Infrast & tructure & \\
\hline BASRAH & 23.73 & 72.5 & \multirow{2}{*}{\multicolumn{3}{|c|}{ Requirements for an Expanded Fuel Ethanol }} \\
\hline ILLINOIS WEEKS & 27.31 & 247.9 & & & \\
\hline KANSAS COMMON & 26.39 & 10.77 & \multicolumn{3}{|c|}{ to 4.3 bgy } \\
\hline KUWAIT & 23.58 & 52.98 & \multirow{3}{*}{\multicolumn{3}{|c|}{$\begin{array}{l}\text { *121 MBD ethanol use } \\
\text { *Assume all RFG contains ethanol }\end{array}$}} \\
\hline LOUISIANA GULF & 26.12 & 338 & & & \\
\hline MAYA & 19.73 & 82.68 & & & \\
\hline MISSISSIPPI BAXTER & 23.17 & 53.83 & \multicolumn{3}{|c|}{ *Add +1 cpg for des of gasolines: } \\
\hline MISSISSIPPI LATHEY & 23.56 & 53.83 & CONVENTIONAL GASOLINE & 37.72 & 1132 \\
\hline MONTANA/WYOMING & 27.64 & 2.5 & CG WITH ETHANOL & 37.72 & 880. \\
\hline BONNY LIGHT & 26.03 & 21.42 & REFORMULATED GASOLINE & 39.92 & 330.7 \\
\hline NIG MEDIUM & 21.2 & 188.2 & AVIATION GAS & 45.63 & 6.29 \\
\hline OKLAHOMA CEMENT & 25.64 & 95.86 & \multirow{2}{*}{\multicolumn{3}{|c|}{$\begin{array}{l}\text { *Distillate volumes adjusted for constant } \\
\text { energy service provided }\end{array}$}} \\
\hline $\begin{array}{ll}\text { SAUDI } & \text { HEAVY } \\
\text { SAUDI } & \text { LIGHT }\end{array}$ & $\begin{array}{l}22.35 \\
24.97\end{array}$ & $\begin{array}{l}121.3 \\
121.3\end{array}$ & \multicolumn{2}{|c|}{$\begin{array}{l}\text { energy service provided } \\
\text { MIL JFT FUEL JP8 }\end{array}$} & \\
\hline TEXAS EAST HAWKINS & 23.64 & 383.1 & $\begin{array}{l}\text { MIL JET FUEL JP8 } \\
\text { COMMERCIAL JET A /KERO }\end{array}$ & $\begin{array}{l}30.60 \\
30.56\end{array}$ & $\begin{array}{l}7.591 \\
292.5\end{array}$ \\
\hline TEXAS GULF INTERMED & 28.29 & 663. & 2D VIA ADVANCE TECH 1 & 31.74 & $\geq 0$ \\
\hline TEXAS WEST SOUR & 26.15 & 663. & 2D ON-HIGHWAY DIESEL & 31.74 & $\geq 0$ \\
\hline UK NORTH SEA & 25.31 & 59.2 & 2D VIA ADVANCED TECH 2 & 31.74 & $\geq 0$ \\
\hline UTAH & 27.31 & 5.341 & *ON-ROAD DIESEL PRODUCTION & SUMS TO 86 & $65.3 \mathrm{MBD}$ \\
\hline VENEZ LIGHT/LOT17 & 24.12 & 0.995 & RAILROAD DIESEL & 31.69 & 16.53 \\
\hline VENEZ JOBO & 15.27 & 331.4 & HEATING OIL (NO2) & 31.69 & 23.64 \\
\hline VENEZ MEDIUM/TJMED & 21.15 & 16.16 & OTHER DIESEL & 31.69 & 218.1 \\
\hline \multirow[t]{2}{*}{ WYOMING SOUR } & \multirow[t]{2}{*}{22.59} & \multirow[t]{2}{*}{191.1} & RESIDUAL $0.3-0.7 \%$ & 18.41 & 7.17 \\
\hline & & & NET RESIDUAL $0.7-1.0 \%$ & 17.69 & 26.85 \\
\hline NATURAL GAS (FOE) & 20.17 & $\geq 0$ & NAPHTHA TO P.CHEM & 25.47 & $\geq 0$ \\
\hline NATURAL GASOLINE & 31.06 & $\leq 48.0$ & *Sinor (2002): & & \\
\hline ISOBUTANE & 30.49 & $\leq 37.38$ & AROMATICS TO P. CHEM & 39.46 & $\geq 0$ \\
\hline NORMAL BUTANE & 25.94 & $\leq 8.12$ & GAS OIL TO PETROCHEM & 29.96 & $\geq 0$ \\
\hline ETHANOL TO CG & 37.72 & $\geq 0$ & NET SPECIAL NAPHTHAS & 25.47 & $\geq 0$ \\
\hline ETHANOL TO RFG & 37.72 & $\geq 0$ & *PETROCHEMICAL PRODUCTION & SUMS TO 73.6 & 6 MBD \\
\hline NAPHTHA/REF FEED & 36.53 & $\leq 13.55$ & LUBES \& WAXES & 36.94 & 32.7 \\
\hline GAS OIL LOW SULFUR & 30.04 & $\leq 6.775$ & *DOE (1998): & & \\
\hline GAS OIL HIGH SULFUR & 29.63 & $\leq 6.775$ & COKE ST/CD LOW SULF & 9.993 & $\geq 0$ \\
\hline DIESEL IGN IMP & 402.2 & $\geq 0$ & COKE ST/CD HIGH SULF & 4.996 & $\geq 0$ \\
\hline & & & ROAD OIL \& ASPHALT & 21.27 & $\geq 0$ \\
\hline & & & ASPHALT FROM SDA & 21.27 & $\geq 0$ \\
\hline & & & $\begin{array}{l}\text { *ASPHALT AND RESID PRODUCTION } \\
\text { *SUL PRICE FROM SWain }(199\end{array}$ & SUMS TO 293. & \\
\hline & & & SULFUR & 23.64 & $\geq 0$ \\
\hline
\end{tabular}




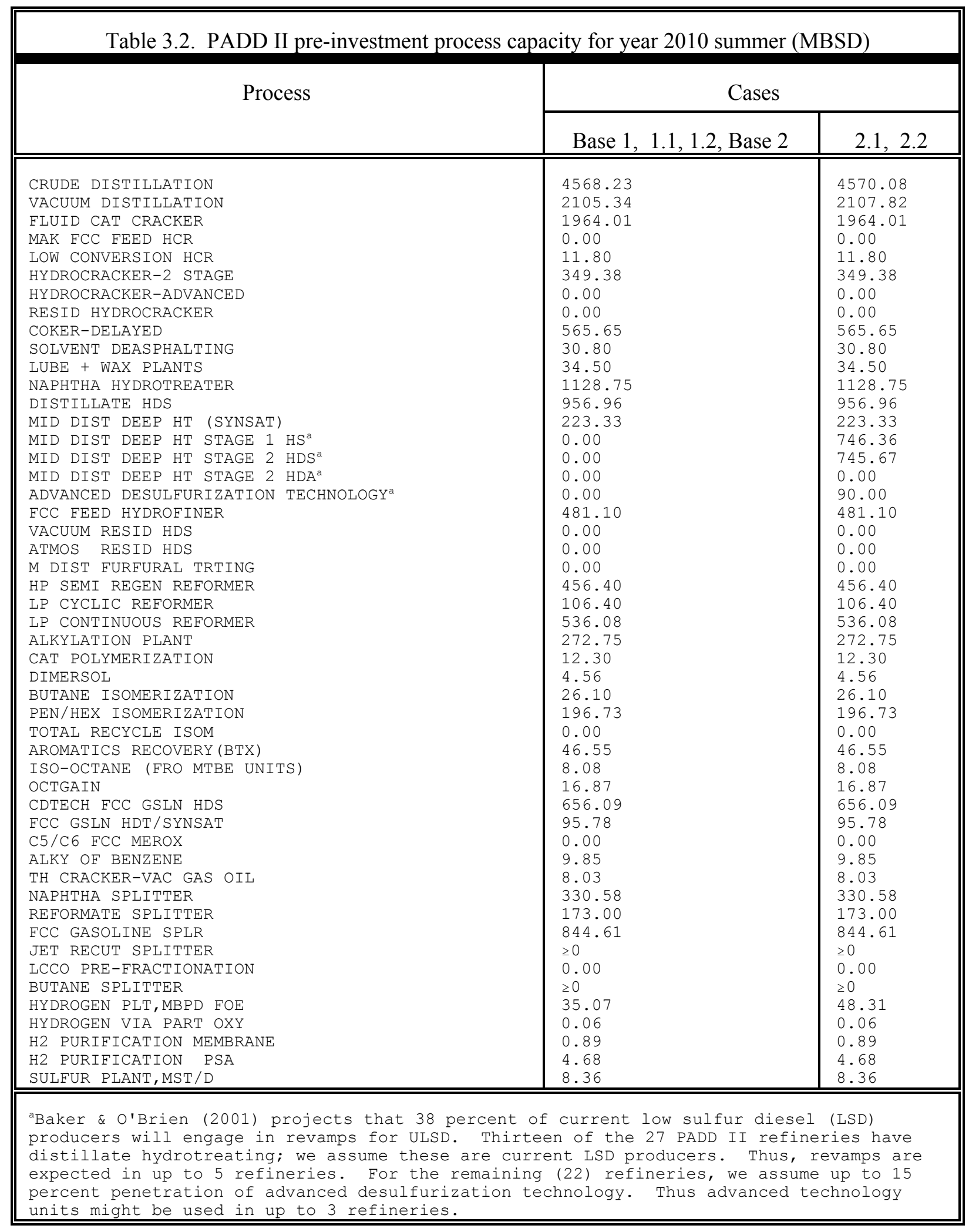




\section{I-3.3.1 Refinery Production Rates}

Refinery summer production rates are based on the Petroleum Supply Annual 2000 (DOE 2001b). Refinery production is projected to year 2010, using the Reference Case growth rates published in Table A11 of the Annual Energy Outlook 2002 with Projections to 2020 (DOE 2001a).

\section{I-3.3.2 Distillate Quality}

On-road diesel fuel has sulfur, cetane number, aromatics content and emissions specifications (nominal and actual) as shown in Table 3.3. The actual specifications for cetane number and aromatics content are based on refiner guidance that the lowest reasonable blending margins should be based on the reproducibility of tests that determine property values. Reproducibility is the variability of the average values obtained by several test operators while measuring the same item. We assume a 3.8 number margin for our cetane number specifications, based roughly on the reproducibility of ASTM D613. For aromatics, the reproducibility of ASTM D1319 is $2.5 \mathrm{vol}$ percent when total aromatics content is approximately $15 \mathrm{vol}$ percent. We have no basis for assumptions for additional margins that may be needed for pipeline degradation of product quality.

Off-road diesel fuels (including No. 2 home heating oil) have maximum allowable sulfur contents of $500 \mathrm{ppm}$ nominal, which is modeled as $350 \mathrm{ppm}$ maximum actual. As discussed in Section I-3.1, a volume of diesel fuel equal to the off-road volume is assumed to be at a sulfur content of $500 \mathrm{ppm}$ (nominal) and the rest at 15 ppm sulfur (i.e., ULSD) in year 2010, but the actual end use markets for the two sulfur levels may be more mixed than that. The critical implied assumption is that all high sulfur (greater than $500 \mathrm{ppm}$ ) diesel fuel disappears from the refinery slate by 2010, in PADD II. Otherwise, specifications for diesel fuels, distillates and products other than gasoline are based on API/NPRA (1997) and NPC (1993). On-road dieselequivalent miles-per-gallon (mpg) are held constant across cases. 


\begin{tabular}{|c|c|c|c|c|c|c|c|}
\hline \multirow[t]{2}{*}{ Case } & \multirow[b]{2}{*}{ Investment } & \multicolumn{6}{|c|}{ On-road diesel fuel properties ${ }^{\mathrm{a}}$} \\
\hline & & Fuel category & $\begin{array}{l}\text { Sulfur } \\
\text { ppm } \\
\max \\
\end{array}$ & $\begin{array}{c}\text { Cetane } \\
\text { number } \\
\text { min }\end{array}$ & $\begin{array}{c}\text { Aromatics } \\
\text { vol } \% \\
\max \\
\end{array}$ & NOx reduction ${ }^{\mathrm{d}}$ & PM reduction \\
\hline Base Case 1 & $\begin{array}{c}\text { Sunk } \\
\text { @ Base Case 1 }\end{array}$ & Low sulfur diesel & $\begin{array}{l}500^{\mathrm{b}} \\
350^{\mathrm{c}}\end{array}$ & 40 & NA & NA & NA \\
\hline Case 1.1 & $\begin{array}{c}\text { Sunk } \\
\text { @Base Case } 1\end{array}$ & $\begin{array}{l}\text { ULSD/Vehicle } \\
\text { performance }\end{array}$ & $\begin{array}{c}15^{\mathrm{b}} \\
8^{\mathrm{c}}\end{array}$ & $\begin{array}{c}55^{\mathrm{b}} \\
58.8^{\mathrm{c}}\end{array}$ & $\begin{array}{c}15^{\mathrm{b}} \\
12.5^{\mathrm{c}}\end{array}$ & NA & NA \\
\hline Case 1.2 & $\begin{array}{c}\text { Sunk } \\
\text { @ Base Case 1 }\end{array}$ & $\begin{array}{l}\text { ULSD/Emissions } \\
\text { reduction }\end{array}$ & $\begin{array}{l}15^{\mathrm{b}} \\
8^{\mathrm{c}}\end{array}$ & 40 & NA & $\begin{array}{c}8.3 \%: \\
\text { Determined } \\
\text { from Case } 1.1\end{array}$ & $\begin{array}{c}19.8 \% \text { : } \\
\text { Determined } \\
\text { from Case } 1.1\end{array}$ \\
\hline Base Case 2 & $\begin{array}{c}\text { Sunk } \\
\text { @Base Case } 1\end{array}$ & ULSD & $\begin{array}{c}15^{\mathrm{b}} \\
8^{\mathrm{c}}\end{array}$ & 40 & NA & NA & NA \\
\hline Case 2.1 & $\begin{array}{c}\text { Sunk } \\
@ \text { Base Case } 2\end{array}$ & $\begin{array}{l}\text { ULSD/Vehicle } \\
\text { performance }\end{array}$ & $\begin{array}{l}15^{\mathrm{b}} \\
8^{\mathrm{c}}\end{array}$ & $\begin{array}{c}55^{\mathrm{b}} \\
58.8^{\mathrm{c}}\end{array}$ & $\begin{array}{c}15^{\mathrm{b}} \\
12.5^{\mathrm{c}}\end{array}$ & NA & NA \\
\hline Case 2.2 & $\begin{array}{c}\text { Sunk } \\
\text { @Base Case } 2\end{array}$ & $\begin{array}{l}\text { ULSD/Emissions } \\
\text { reduction }\end{array}$ & $\begin{array}{c}15^{\mathrm{b}} \\
8^{\mathrm{c}}\end{array}$ & 40 & NA & $\begin{array}{c}8.2 \%: \\
\text { Determined } \\
\text { from Case } 2.1\end{array}$ & $\begin{array}{c}19.8 \% \\
\text { Determined } \\
\text { from Case } 2.1\end{array}$ \\
\hline $\begin{array}{l}\text { Sensitivity } \\
\text { Cases }\end{array}$ & \multicolumn{7}{|c|}{$\begin{array}{l}\text { Refinery demand curves estimated parametrically for a Canadian synthetic crude oil in all cases except Case } \\
2.1 \text { and 2.2. }\end{array}$} \\
\hline \multicolumn{8}{|c|}{ 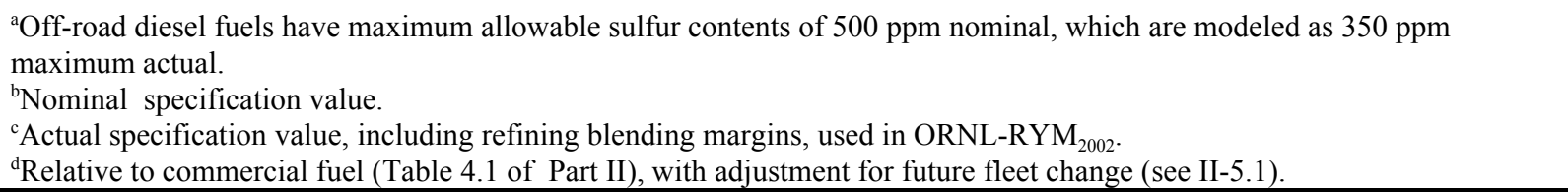 } \\
\hline
\end{tabular}




\section{I-3.3.3 Gasoline Quality}

To the extent possible, gasoline properties are consistent with key provisions of proposed U.S. Senate Bill S.517 (subsequently amended into U.S. House of Representatives Bill H.R.4.) which, among other initiatives:

1. Specifies the Renewable Fuels Standard requirement (4.3 billion gallons for the U.S. in year 2010).

2. Ensures that 35 percent or more of the quantity of the renewable fuels requirement is used during each of two specified seasons. We assume uniform use of ethanol across the seasons.

3. Prohibits the use of MTBE, not later than four years after the date of enactment.

4. Eliminates the oxygen content requirement for RFG.

5. Maintains Toxic Air Pollutant emission reductions for RFG at 1999-2000 baseline levels.

6. Consolidates the Volatile Organic Compound (VOC) emissions specification for all RFG to the more stringent requirement for southern RFG.

For those areas not otherwise specified by S.517:

RFG satisfies Phase II Federal recipe and emissions requirements for PADD II.

There are no VOC offset allowances for RFG in the summer of 2010. (For example, RFG blended with 10 percent ethanol and sold in Chicago and Milwaukee has had a 2 percent offset in the VOC requirement, based on carbon monoxide reduction benefits).

Conventional gasoline (CG) without oxygenates satisfies requirements for Phase II Reid vapor pressure (RVP) volatility control and antidumping.

CG with 10 vol percent ethanol has a 1 psi RVP waiver and satisfies requirements for antidumping. For this study, we assume that governors of PADD II states will not exercise the S.517 option to repeal the 1 psi RVP waiver.

RFG and CG satisfy the toxics anti-backsliding rulemaking of 2001 (EPA 2001). Incremental RFG satisfies one of two possible toxics standards. If incremental RFG is produced by refineries currently producing RFG, then the standard for incremental production is 21.5 percent toxics reduction. If incremental RFG is from refineries not currently producing RFG, then the standard for incremental production is 25.3 percent toxics reduction.

All gasolines contain no more than $30 \mathrm{ppm}$ sulfur, on average.

Ethanol usage patterns are based on Infrastructure Requirements for an Expanded Fuel Ethanol Industry (DAI 2002).

Gasoline properties are weighted to reflect the market requirements (e.g., Class splits) implied in recent data provided by EPA (Weihrauch 2001).

The production share of RFG is based on monthly production shares reported in the Petroleum Supply Annual 2000 (DOE 2001b). Gasoline-equivalent mpg are held constant across cases. Where required, gasolines are pooled to combine volumes and properties of regular, mid-grade, and premium grades. The 
source data for octane estimates and for pooling are the 1996 American Petroleum Institute/National Petroleum Refiners Association Survey of Refining Operations and Product Quality (API/NPRA 1997) and the NPRA Survey of U.S. Gasoline Quality and U.S. Refining Industry Capacity to Produce Reformulated Gasolines (NPRA 1991).

\section{I-3.3.4 Refinery Raw Materials}

Refinery inputs of crude oil and raw materials are based on Petroleum Supply Annual 2000 (DOE 2001b).

Refinery inputs are projected to year 2010, using the Reference Case growth rates published in Table A11 of the Annual Energy Outlook 2002 with Projections to 2020 (DOE 2001a). The crude oil mix is based on the regional mixes reported by NPC (1993).

\section{I-3.3.5 Refinery Capacity and Investment}

Refinery capacity is based on in-place capacity and construction as reported in Refinery Capacity Data as of January 1, 2001 (DOE 2002b), NPC (1993), NPRA (1991), the Oil \& Gas Journal (Stell 2001a,b), or American Petroleum Institute and NPRA (API/NPRA 1997). Capacities for reformate splitter, fluid catalytic cracker (FCC) naphtha splitter, and straight run naphtha splitter are set at the greatest of capacity reported in NPRA (1991); or NPC (1993); or unconstrained Base Case 1 capacity (see Table 3.2). The loss of hydrogen to fuel gas is based on API/NPRA (1997).

Process capacity investment is based on a nominal 15 percent after-tax discounted cash flow rate of return on investment (ROI), and actual investment cost is based on an actual 10 percent after-tax discounted cash flow ROI. For existing capacity, typical investment costs are used for up to 20 percent expansion in capacity. For capacity greater than the defined expansion limit, investment is subject to economies of scale, according to the "six-tenths factor" relationship:

$$
\operatorname{Cost}_{\text {New }}=\left(\text { Capacity }_{\text {New }} / \text { Capacity }_{\text {Typical Size }}\right)^{n *} \operatorname{Cost}_{\text {Typical Size }} \text {, with } \mathrm{n} \text { between } 0.6 \text { and } 0.7
$$

New capacity is averaged over the affected refineries. Investment options include established technologies, plus the new process options described in Section I-2.2.

\section{I-3.3.6 Product Revenue and Raw Material Costs}

Revenues and costs are expressed in year 2000 U.S. dollars. Raw material and crude oil costs are based on the Annual Energy Outlook 2002 with Projections to 2020 (DOE 2001a), NPC (1993), Petroleum Marketing Annual (DOE 2002a), and guidance from the Energy Information Administration (EIA). Product prices are based on the Annual Energy Outlook 2002 with Projections to 2020 (DOE 2001a), Petroleum Marketing Annual (DOE 2002a), historical price differentials, price ratios, heating values, estimates reported by NPC (1993), and EIA guidance.

\section{I-3.3.7 Study Cases}

The two different on-road diesel fuel reformulation pathways, with equal emissions performance requirements, are defined as follows:

Cetane number (55 minimum) and aromatics specifications (15 vol percent maximum) are those recommended by the World Wide Fuel Charter. We categorize this RFD as a "vehicle performance 
fuel," as discussed in Section I-3.1. The NOx and PM emissions properties of this fuel are estimated with an eigenfuel model.

Emissions are specified not to exceed the NOx and PM emissions of the vehicle performance fuel. We categorize this RFD as an "emissions reduction fuel," as discussed inn Section I-3.1. This fuel otherwise conforms to nominal ASTM D975 specifications for No. 2-D, with a minimum cetane number of 40 and with no specification for aromatics content. These nominal specifications are converted to actual specifications according to Section I-3.3.2.

For each of the two different reformulation pathways, two different investment pathways are investigated, in order to understand the refinery economic benefits of early notice of requirements for more stringent cetane number and aromatics specifications. The two different investment pathways are:

Parallel pathway: There is early notice of requirements for RFD, and capital investments have not yet been made to satisfy the ULSD requirement of year 2006.

Sequential pathway: There is delayed notice of requirements for RFD, and capital investments have already been sunk to satisfy the ULSD requirement.

The study cases are summarized in Table 3.3, which also shows sensitivity cases to estimate the refinery demand curves for a Canadian synthetic crude oil. The ORNL-RYM ${ }_{2002}$ analysis focuses on refinery cost changes, diesel fuel oil blend stocks, diesel fuel oil properties, and process capacity investment. This report contains tabulated results and discussions for:

Overall model results for calculation of cost change

Properties of diesel fuels (on road and off road)

Blendstocks for diesel fuels

Properties of gasolines

Blendstocks for gasoline

Raw material and product volume balance

Hydrogen balance and utilization of key process units

Process investments

Components of cost change

Quality of crude oil processed

Energy use 



\section{I-4. EMISSIONS MODELING}

\section{I-4.1 GASOLINE}

ORNL-RYM ${ }_{2002}$ represents gasoline blending to satisfy both formula and emissions standards for gasolines. Emissions modeling provides a means for predicting the emissions performance of a gasoline, given other properties of the gasoline. The EPA Complex Model is a set of non-linear equations that predicts emissions of VOCs, toxic air pollutants (TAPs), and NOx in terms of gasoline properties including RVP, E200, E300, benzene, oxygen, sulfur, aromatics, and olefins contents (Korotney 1993). The non-linear Complex Model presents difficult adaptation problems for use in refinery linear programs. Each gasoline blending component has VOC, TAP, and NOx blending values that vary with overall gasoline composition. The Complex Model is represented in ORNL-RYM 2002 by a linear delta method in which off-line software computes coefficients of change in emissions with changes in a gasoline property. These coefficients are then used in the off-line software to compute emissions blending values for the gasoline blending components. ORNL-RYM ${ }_{2002}$ is solved iteratively, until convergence of the objective function value is achieved.

\section{I-4.2 DIESEL FUEL}

PART II of this report provides a detailed discussion of the implementation of eigenfuel-based diesel fuel emissions models for ORNL-RYM ${ }_{2002}$. The eigenfuel method overcomes the deficiencies of emissions models derived from multiple regression analysis. Multiple regression analysis is widely used for expressing the dependence of a response variable on several predictor variables. In spite of its evident success in many applications, multiple regression analysis can face serious difficulties when the predictor variables are to any appreciable extent covariant (McAdams, Crawford and Hadder 2002). Efforts to evaluate the separate effects of fuel variables on the emissions from heavy-duty diesel engines are often frustrated by the close association of fuel properties.

Most heavy-duty diesel engine research has been conducted with test fuels "concocted" in the laboratory to vary selected fuel properties in isolation. While it might eliminate the confounding effect caused by naturally covarying fuel properties, this approach departs markedly from the real world, where reformulation of fuels to reduce emissions will naturally and inevitably lead to changes in a series of interrelated properties. To address these concerns, we have implemented the alternative eigenfuel-based approach to modeling the effects of fuel characteristics on emissions.

The eigenfuel approach is based on the use of Principal Components Analysis to describe fuels in terms of eigenvectors. Each eigenvector represents a unique and mathematically independent characteristic of diesel fuel. Because of their relationships with refinery processing and blending, these eigenvectors are called eigenfuels in our work. As demonstrated in McAdams, Crawford and Hadder (2000a,b and 2002), eigenfuels have many advantages, including:

Simplification of the analysis, because the mathematical independence of eigenfuels eliminates correlations among the variables and the complications introduced by multi-collinearity.

Economy of representation, because a smaller number of such vector variables may effectively replace a larger number of original variables.

Greater understanding of the patterns of variation that are important to emissions, and how these patterns relate to refinery processing and blending. 
New insight into the optimal economic formulation of fuels to reduce emissions.

Knowing the extent of interdependence among fuel variables, we should not be surprised by the difficulty of selecting an "optimal" set of variables for a regression model. We may believe that cetane number or density has an important influence on emissions, but either may be nearly replaced by a combination of other variables. Stepwise regression, a commonly used multiple regression analysis technique, searches through a sequence of differing model formulations to find one that is "optimum." With diesel fuel test data, there can be many different sets of variables that perform nearly as well as the one set ultimately chosen.

Fig. 1 brings this point into focus. There are 4,095 different regression models that can be formed from twelve fuel properties, and these models form the universe among which stepwise regression searches. It will take all twelve properties to place a model at the very end of the curve. Forty-five different models populate the last two percent of fuels-related variance in NOx, and these models typically involve 7 to 9 fuel property variables. The coefficients of the fuel property variables can be significantly different among these models, leading to uncertainty about their relative importance in testing and in the cost-effective blending of refinery stocks. However, models based on a small number of eigenfuels perform well, with five eigenfuels explaining nearly 97 percent of the fuels-related variance in NOx. The eigenfuel coefficients provide an unambiguous basis for cost-effective blending of refinery stocks.

We recognize that the existing test data for heavy-duty diesel engines are inadequate to answer fully the many questions related to the effect of fuels on emissions. Given this limitation, however, we find that the eigenfuel approach leads to new perspectives on diesel fuel-emissions relationships:

Fuel properties are only surrogate variables for underlying causal factors. Much of the emissions reduction seen in past testing comes from reducing highly aromatic cracked stocks in diesel fuel. Because these stocks are low in cetane number and high in density, researchers have tended to attribute the emissions reductions to the increase in cetane number or reduction in density associated with their removal, rather than to the compositional change itself.

How one varies a fuel property can be the most important factor in determining the emissions response. A given fuel property can be changed in many ways, and a unit change in that property can produce markedly different effects on emissions depending on how that change is introduced.

\section{I-4.3 DIESEL FUEL EMISSIONS MODELING CONCERNS}

During the diesel fuel reformulation study, premises and progress were reviewed with the American Petroleum Institute Economics Work Group and the Diesel Issues Subcommittee. With regard to emissions modeling, API raised the following concerns: data are insufficient and inappropriate for estimation of an emissions model; it is inappropriate to assume that percent reductions of emissions in the current fleet will apply equally to future engine-aftertreatment systems; diesel engines with exhaust gas recirculation may have a NOx response to cetane that is directionally different from the data used in eigenfuel-based model development; lube oil slippage could have significant effects on emissions.

Appendices I-C and I-D include API comments and our responses to API concerns. Part II of this report explains our resolution of some of the API concerns. 
Figure 4.1. Comparative Performance of Regression Models (based on twelve fuel properties)

Y-axis is percent of fuels-related variance in NOx

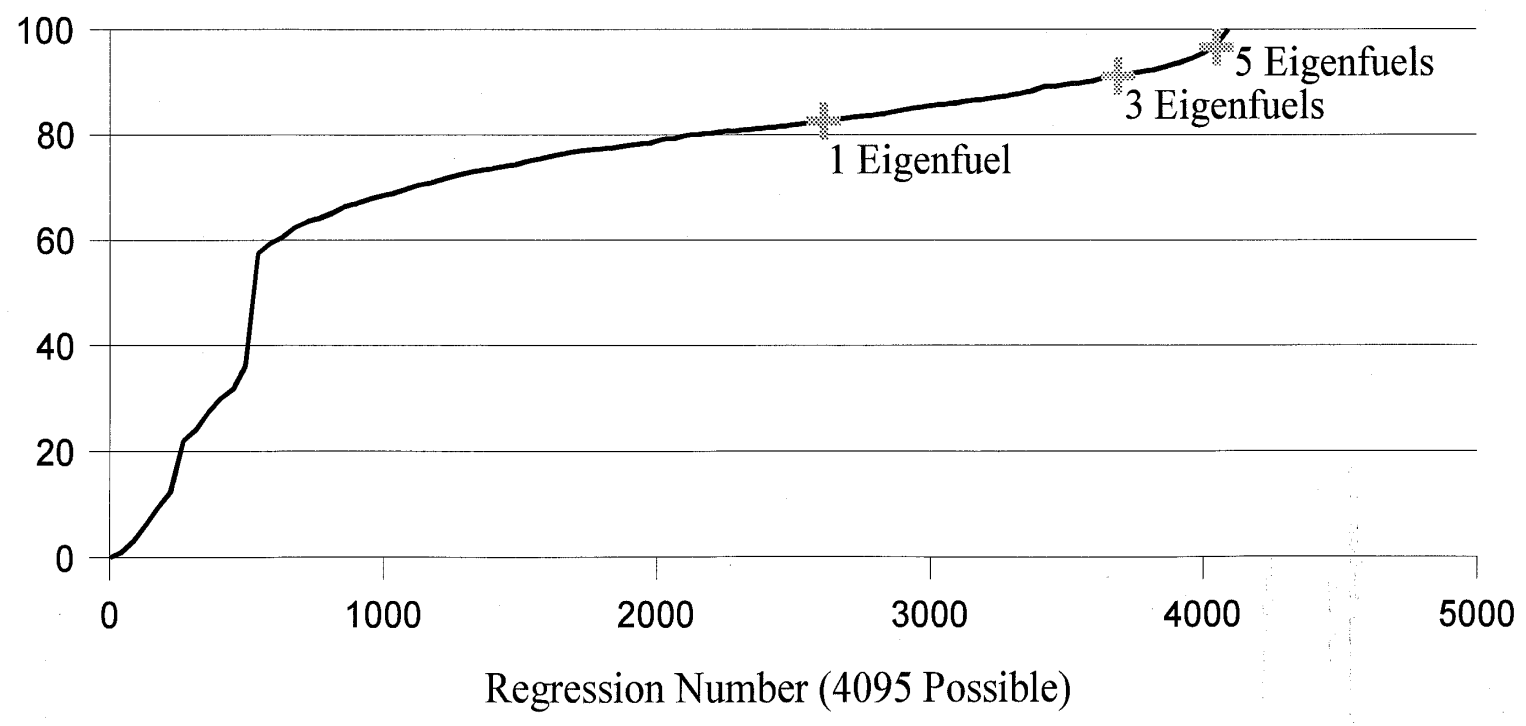





\section{I-5. CALIBRATION AND DEMONSTRATION}

\section{I-5.1 CALIBRATION CRITERIA}

Prior to executing the study cases, we calibrated ORNL-RYM $\mathrm{M}_{2002}$ for approximate replication of PADD II year 2000 summer data for gasoline and distillate production and process utilization. These model adjustments were then used in the study cases themselves. The calibration criteria included the following:

Crude Oil: Model-derived crude oil sulfur content and gravity were to be within 10 percent of the year 2000 data source (DOE 2001b).

Process Utilization: The NPC (1993) criterion of "maximum variance of \pm 8 percent" was used for utilization of the four process types reported in the year 2000 data source (DOE 2001b).

Gasoline: Model-derived gasoline volume and property values were to be either (1) within 10 percent of the data source (EPA 2002), or (2) within the calibration criteria percentages reported by MathPro in "U.S. Petroleum Refining" (NPC 2000).

Distillates: API/NRPA (1997) distillate quality information may be outdated for a year 2000 distillate property calibration. However, shadow values for distillate products were evaluated, based on the NPC (1993) criterion of a maximum variance of \pm 20 percent, in comparison with refiner prices for products reported for year 2000 (DOE 2002a).

\section{I-5.2 CALIBRATION ADJUSTMENTS}

Data sources and inputs (without extrapolation to a future year) for the calibration are listed in the Section I-3 premises. The required adjustments in ORNL-RYM ${ }_{2002}$ were determined to be:

Fix distillation properties of gasolines to approximate historical values. This adjustment objective is similar to distillation curve calibration performed by MathPro in NPC (2000).

Scale aromatics blending values of gasoline blendstocks by 0.93 (except for high aromatics blendstocks). ORNL-RYM 2002 blendstock property values are primarily based on PADD III (U.S. Gulf Coast) data, and adjustments are required for modeling other regions. API/NRPA (1997) reported that the gasoline pool aromatics content for PADD II was 0.92 of the gasoline pool aromatics content for PADD III.

Scale olefin blending values of gasoline blendstocks by 0.65 (except for high olefin blendstocks). ORNL-RYM 2002 blendstock property values are primarily based on PADD III data, and adjustments are required for modeling other regions. API/NRPA (1997) reported that the gasoline pool olefins content for PADD II was 0.81 of the gasoline pool olefins content for PADD III.

Apply blending ratio controls to FCC gasoline blendstocks. In the year 2000 calibration, ratio controls are also applied to FCC gasoline feed to the FCC gasoline splitter and to light and heavy FCC gasoline blended to finished gasolines. These ratios result in a reasonable distribution of olefins over different gasoline types.

In the year 2000 calibration, production constraints were relaxed by targeting product outputs (gasolines and diesel fuels) at \pm 5 percent of the observed year 2000 levels. 


\section{I-5.3 CALIBRATION RESULTS}

Tables 5.1 through 5.5 show that, with the ORNL-RYM ${ }_{2002}$ calibration adjustments, the calibration criteria were satisfied.

\begin{tabular}{||c|c|c|c||}
\hline \multicolumn{3}{|c||}{ Table 5.1. Crude oil quality calibration demonstration } \\
for PADD II summer \\
\hline Parameter & $\begin{array}{c}\text { Observed for } \\
\text { year 2000 }\end{array}$ & $\begin{array}{c}\text { Calibration for } \\
\text { year 2000 }\end{array}$ & $\begin{array}{c}\text { Calibration difference } \\
\text { versus year 2000, } \\
\text { percent }\end{array}$ \\
\hline \hline $\begin{array}{l}\text { Sulfur content, wt } \\
\text { percent }\end{array}$ & 1.30 & 1.27 & -2.3 \\
\hline Gravity, $\mathrm{API}^{\circ}$ & 32.9 & 32.3 & -1.8 \\
\hline
\end{tabular}

\begin{tabular}{|c|c|c|c|}
\hline \multicolumn{4}{|c|}{$\begin{array}{c}\text { Table 5.2. Process unit utilization calibration demonstration } \\
\text { for PADD II summer }\end{array}$} \\
\hline Process unit & $\begin{array}{c}\text { Observed } \\
\text { utilization for } \\
\text { year } 2000, \\
\text { percent }\end{array}$ & $\begin{array}{c}\text { Calibration } \\
\text { utilization for } \\
\text { year } 2000, \\
\text { percent } \\
\end{array}$ & $\begin{array}{c}\text { Calibration difference } \\
\text { versus year } 2000, \\
\text { percent }\end{array}$ \\
\hline $\begin{array}{l}\text { Atmospheric crude oil } \\
\text { distillation }\end{array}$ & 93.4 & 90.1 & -3.6 \\
\hline Catalytic cracking & 93.5 & 91.0 & -2.7 \\
\hline $\begin{array}{l}\text { Catalytic } \\
\text { hydrocracking }\end{array}$ & 97.7 & $97.7(\mathrm{UL})^{\mathrm{a}}$ & 0 \\
\hline Delayed coking & 88.5 & 88.5 (UL) & 0 \\
\hline
\end{tabular}




\begin{tabular}{|c|c|c|c|}
\hline \multicolumn{4}{|c|}{$\begin{array}{l}\text { Table 5.3. Reformulated gasoline calibration demonstration } \\
\text { for PADD II summer }\end{array}$} \\
\hline Parameter & Observed value & Calibration value & $\begin{array}{c}\text { Calibration difference, } \\
\text { percent }\end{array}$ \\
\hline Volume, MBD & 270 & $257(\mathrm{LL})^{\mathrm{a}}$ & -5.0 \\
\hline Marginal cost, $\$ / b b l$ & 47.44 & 50.57 & +6.6 \\
\hline RVP, psi & 6.7 & 6.5 & -3.0 \\
\hline E200, percent & 45.2 & 42.2 & -6.6 \\
\hline E300, percent & 85.7 & 84.9 & -0.9 \\
\hline Aromatics, vol percent & 18.5 & 18.7 & +1.1 \\
\hline Benzene, vol percent & 0.80 & 0.80 & 0.0 \\
\hline Olefins, vol percent & 3.6 & $3.8(\mathrm{LL})$ & +5.6 \\
\hline Sulfur, ppm & 86 & 89 & +3.4 \\
\hline Specific gravity & 0.742 & 0.747 & +0.7 \\
\hline Oxygen & \multicolumn{3}{|c|}{ Oxygen content/oxygenates fixed per EPA (2002) } \\
\hline Summer Total Toxics, $\mathrm{mg} / \mathrm{mi}$ & 58.6 & $58.6(\mathrm{UrL})^{\mathrm{b}}$ & 0.0 \\
\hline $\mathrm{NOx}, \mathrm{mg} / \mathrm{mi}$ & 1160 & $1160(\mathrm{UrL})$ & 0.0 \\
\hline $\mathrm{VOC}, \mathrm{mg} / \mathrm{mi}$ & 1014 & $1011(\mathrm{UrL})$ & -0.3 \\
\hline \multicolumn{4}{|c|}{ aLL: Parameter at upper limit in model specifications. } \\
\hline
\end{tabular}




\begin{tabular}{|c|c|c|c|}
\hline \multicolumn{4}{|c|}{$\begin{array}{l}\text { Table 5.4. Conventional gasoline calibration demonstration } \\
\text { for PADD II summer }\end{array}$} \\
\hline Parameter & Observed value & Calibration value & $\begin{array}{c}\text { Calibration difference, } \\
\text { percent }\end{array}$ \\
\hline Volume, MBD & 1556 & 1479 & -5.0 \\
\hline Marginal cost, $\$ / b b l$ & 44.79 & 48.52 & +8.3 \\
\hline RVP, psi & 8.1 & $8.1(\mathrm{UrL})^{\mathrm{a}}$ & 0.0 \\
\hline E200, percent & 47.0 & $48.9(\mathrm{UL})^{\mathrm{b}}$ & +4.0 \\
\hline E300, percent & 81.8 & 83.3 (UL) & +1.8 \\
\hline Aromatics, vol percent & 28.8 & 31.0 & +7.6 \\
\hline Benzene, vol percent & 1.37 & 1.32 & -3.6 \\
\hline Olefins, vol percent & 9.9 & 11.0 & $-11.1^{c}$ \\
\hline Sulfur, ppm & 346 & 316 & -8.7 \\
\hline Specific gravity & 0.746 & 0.747 & +0.1 \\
\hline Oxygen & \multicolumn{3}{|c|}{ Oxygen content/oxygenates fixed per EPA (2002) } \\
\hline Summer Total Toxics, $\mathrm{mg} / \mathrm{mi}$ & 78.7 & 78.8 & +0.1 \\
\hline Summer Exhaust Toxics, mg/mi & 74.3 & $74.6(\mathrm{UrL})$ & +0.4 \\
\hline $\mathrm{NOx}, \mathrm{mg} / \mathrm{mi}$ & 1345 & $1349(\mathrm{UrL})$ & +0.3 \\
\hline \multicolumn{4}{|c|}{$\begin{array}{l}{ }^{a} U r L: \text { Upper regulatory limit. } \\
{ }^{b} \mathrm{UL} \text { : Parameter at upper limit in model specifications. } \\
{ }^{\mathrm{c}} \mathrm{NPC} \text { (2000) calibration difference was } 26.4 \text { percent. }\end{array}$} \\
\hline
\end{tabular}




\begin{tabular}{|c|c|c|c|}
\hline \multicolumn{4}{|c|}{$\begin{array}{l}\text { Table 5.5. Distillate calibration demonstration } \\
\text { for PADD II summer }\end{array}$} \\
\hline Parameter & $\begin{array}{l}\text { Observed value } \\
2000\end{array}$ & $\begin{array}{l}\text { Calibration value } \\
\text { for year } 2000\end{array}$ & $\begin{array}{c}\text { Calibration difference } \\
\text { versus year } 2000, \\
\text { percent }\end{array}$ \\
\hline \multicolumn{4}{|l|}{ Jet A } \\
\hline Volume, MBD & 235.5 & 223.7 & -5.0 \\
\hline Marginal cost, $\$ / b b l$ & 36.70 & 40.86 & +11.3 \\
\hline \multicolumn{4}{|l|}{ On-road diesel fuel } \\
\hline Volume, MBD & 680.2 & 688.8 & +1.3 \\
\hline Marginal cost, $\$ / \mathrm{bbl}$ & 38.11 & 38.11 & 0.0 \\
\hline \multicolumn{4}{|l|}{ Home heating oil } \\
\hline Volume, MBD & 19.3 & 20.28 & +5.0 \\
\hline Marginal cost, $\$ /$ bbl & 38.05 & 33.48 & -12.0 \\
\hline \multicolumn{4}{|l|}{ Railroad diesel fuel } \\
\hline Volume, MBD & 13.5 & 14.14 & +4.7 \\
\hline Marginal cost, $\$ /$ bbl & 38.05 & 30.45 & -20.0 \\
\hline \multicolumn{4}{|l|}{ Other off-road diesel fuel } \\
\hline Volume, MBD & 177.0 & 185.9 & +5.0 \\
\hline Marginal cost, $\$ / \mathrm{bbl}$ & 38.05 & 31.30 & -17.7 \\
\hline
\end{tabular}





\section{I-6. IMPACTS OF DIESEL FUEL REFORMULATION IN PADD II REFINERIES}

The diesel fuel reformulation study estimates the refining impacts of different pathways for reformulation and refinery investment in PADD II, for summer operations in year 2010. Cost, product quality, operational, and investment impacts are determined for the different pathways provided by the cases listed in Table 6.1. This section presents detailed refinery modeling results for the cases.

\begin{tabular}{|c|c|}
\hline Case & On-road diesel upgrading pathway \\
\hline Base Case 1 & $\begin{array}{l}\cdot \text { Low sulfur } \\
\text {-Investment as required }\end{array}$ \\
\hline Case 1.1 & $\begin{array}{l}\cdot \text { ULSD } \\
\text { - Reformulated for vehicle performance } \\
\text { - Early notice of requirements } \\
\text {-Parallel investment }\end{array}$ \\
\hline Case 1.2 & $\begin{array}{l}\text {-ULSD } \\
\text { - Reformulated for emissions reduction } \\
\text {-Early notice of requirements } \\
\text {-Parallel investment }\end{array}$ \\
\hline Base Case 2 & $\begin{array}{l}\text {-ULSD } \\
\text {-Sequential investment (step 1) } \\
\text {-Some newer technologies }\end{array}$ \\
\hline Case 2.1 & $\begin{array}{l}\text {-ULSD } \\
\text { - Reformulated for vehicle performance } \\
\text {-Delayed notice of requirements } \\
\text {-Sequential investment (step 2) }\end{array}$ \\
\hline Case 2.2 & $\begin{array}{l}\text {-ULSD } \\
\text { - Reformulated for emissions reduction } \\
\text { - Delayed notice of requirements } \\
\text {-Sequential investment (step 2) }\end{array}$ \\
\hline
\end{tabular}

\section{I-6.1 BASE CASE 1: LOW SULFUR DIESEL FUEL}

Base Case 1 is one of two base cases used as references to show comparative and cumulative impacts of different reformulation and investment pathways. In Base Case 1, on-road diesel fuel must satisfy current (year 2002) requirements for sulfur content (500 ppm maximum) and cetane number (40 minimum), and there are no requirements for aromatics and emissions quality. However, Base Case 1 is not representative of current quality requirements for off-road diesel fuels (with home heating oil included), which have premised sulfur specifications of $500 \mathrm{ppm}$ maximum. By using this off-road sulfur specification in all cases, we have intentionally removed a variable from the study. Key results for Base Case 1 and all other cases are summarized in Tables 6.2 through 6.15 , which show: 
Overall model results for calculation of cost change (Table 6.2)

Properties of diesel fuels (Table 6.3)

Properties of diesel fuels, by desulfurization technology (Table 6.4)

Blendstocks for diesel fuels (Tables 6.5)

Properties of gasolines (Table 6.6)

Blendstocks for gasoline (Table 6.7)

Pooled blendstocks for gasoline (Table 6.8)

Raw material and product volume balance (Table 6.9)

Hydrogen balance and utilization of key process units (Table 6.10)

Process investments (Tables 6.11 and 6.12)

Components of cost changes (Table 6.13)

Quality of crude oil processed (Table 6.14)

Energy use changes (Table 6.15) 


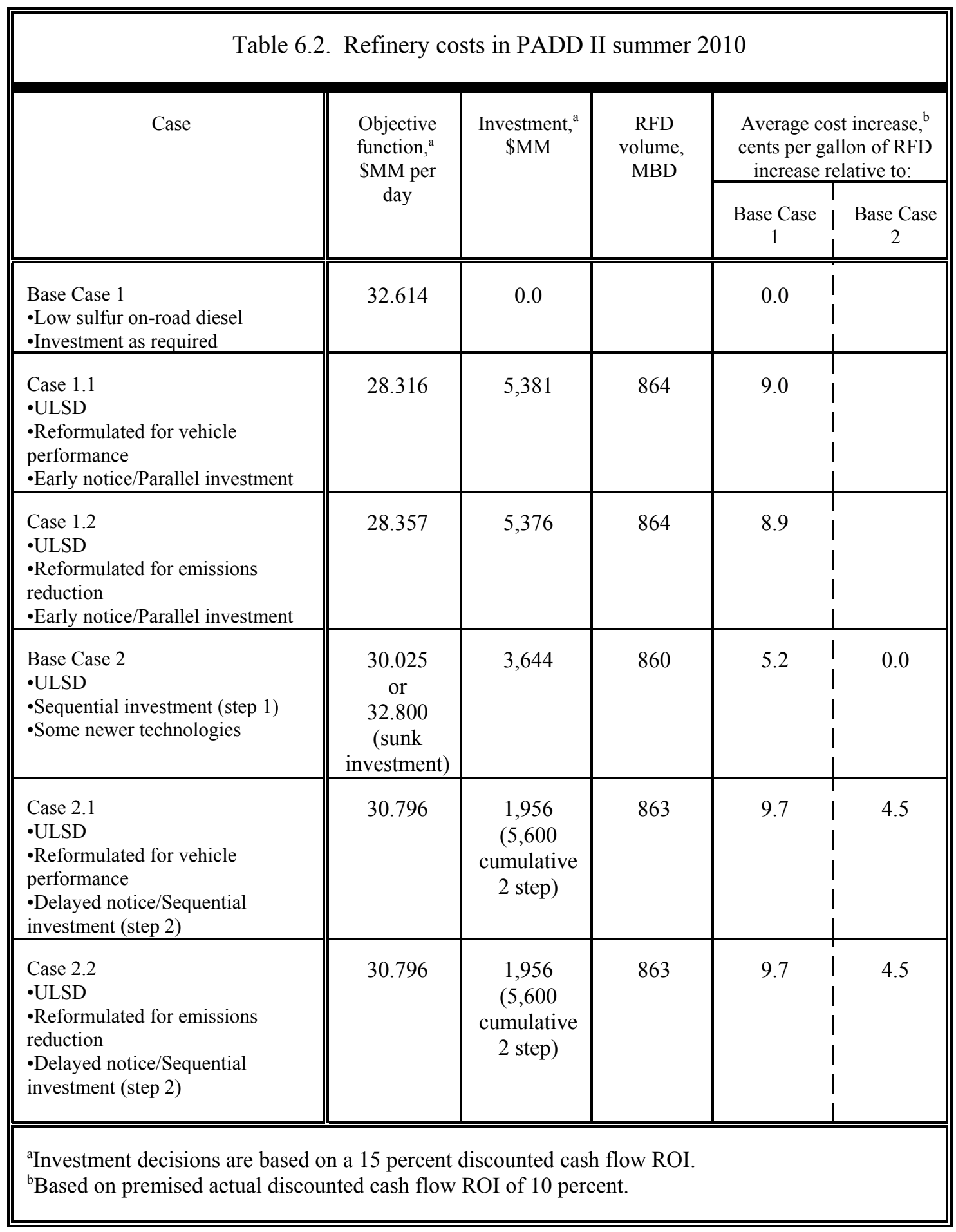




\begin{tabular}{|c|c|c|c|c|c|c|c|c|}
\hline \multirow[t]{2}{*}{ Property } & \multicolumn{4}{|c|}{$\begin{array}{l}\text { Base Case } 1 \\
\cdot \text { - Low sulfur on-road diesel } \\
\text {-Investment as required }\end{array}$} & \multicolumn{4}{|c|}{$\begin{array}{l}\text { Case } 1.1 \\
\text {-ULSD } \\
\text { - Reformulated for vehicle } \\
\text { performance } \\
\text {-Parallel investment }\end{array}$} \\
\hline & $\begin{array}{l}\text { On- } \\
\text { road }\end{array}$ & $\begin{array}{l}\text { Rail- } \\
\text { road }\end{array}$ & $\begin{array}{l}\text { Other } \\
\text { Off- } \\
\text { road }\end{array}$ & $\begin{array}{l}\text { Heating } \\
\text { Oil }\end{array}$ & $\begin{array}{l}\text { On- } \\
\text { road }\end{array}$ & $\begin{array}{l}\text { Rail- } \\
\text { road }\end{array}$ & $\begin{array}{l}\text { Other } \\
\text { Off- } \\
\text { road }\end{array}$ & $\begin{array}{l}\text { Heating } \\
\text { Oil }\end{array}$ \\
\hline Volume, MBD & 844 & 17 & 221 & 24 & 864 & 17 & 218 & 24 \\
\hline API Gravity, ${ }^{\circ}$ API & 35.9 & 37.5 & 35.7 & 37.1 & 39.2 & 34.6 & 33.9 & 33.0 \\
\hline Specific gravity & .8450 & & & & .8291 & & & \\
\hline Sulfur, ppm & 350 & 350 & 350 & 350 & 8 & 260 & 330 & 350 \\
\hline Cetane Index & 48.5 & & & & 53.5 & & & \\
\hline Cetane Number, clear & 44.9 & & & & 51.5 & & & \\
\hline $\begin{array}{l}\text { Cetane Improver, } \\
\text { vol\% }\end{array}$ & .000 & & & & .122 & & & \\
\hline $\begin{array}{l}\text { Cetane Improver, } \\
\text { number increase }\end{array}$ & 0.0 & & & & 7.3 & & & \\
\hline Cetane Number, total & 44.9 & & & & 58.8 & & & \\
\hline Aromatics, vol $\%$ & 26.8 & 21.6 & 24.6 & 22.4 & 12.5 & 23.1 & 29.9 & 28.3 \\
\hline Viscosity, cSt & 2.65 & & & & 2.76 & & & \\
\hline ASTM IBP,${ }^{\circ} F^{a}$ & 377 & & & & 375 & & & \\
\hline $\mathrm{T} 10$ & 436 & 421 & 442 & 437 & 434 & 421 & 440 & 439 \\
\hline $\mathrm{T} 30$ & 463 & 453 & 475 & 468 & 463 & 453 & 473 & 466 \\
\hline $\mathrm{T} 50$ & 499 & 494 & 512 & 499 & 492 & 493 & 508 & 504 \\
\hline $\mathrm{T} 70$ & 549 & 538 & 556 & 538 & 539 & 539 & 554 & 550 \\
\hline $\mathrm{T} 90$ & 589 & 585 & 593 & 588 & 591 & 611 & 612 & 591 \\
\hline $\mathrm{FBP}^{\mathrm{a}}$ & 633 & & & & 635 & & & \\
\hline NOx change, $\%^{\mathrm{b}}$ & -0.7 & & & & -8.3 & & & \\
\hline PM change, $\%^{\mathrm{b}}$ & -2.4 & & & & -19.8 & & & \\
\hline \multicolumn{9}{|c|}{$\begin{array}{l}\text { aIBP and FBP are based on survey reports of differences relative to T10 and T90 (NIPER 1994). } \\
\text { 'Relative to commercial fuel (Table } 4.1 \text { of Part II), with adjustment for future fleet change (see II- } \\
\text { 5.1). }\end{array}$} \\
\hline
\end{tabular}




\begin{tabular}{|c|c|c|c|c|c|c|c|c|}
\hline \multicolumn{9}{|c|}{ Table 6.3 (Continued). Properties of diesel fuels in PADD II summer 2010} \\
\hline \multirow[t]{2}{*}{ Property } & \multicolumn{4}{|c|}{$\begin{array}{l}\text { Case } 1.2 \\
\text {-ULSD } \\
\cdot \text { Reformulated for emissions } \\
\text { reduction } \\
\text {-Parallel investment }\end{array}$} & \multicolumn{4}{|c|}{$\begin{array}{l}\text { Base Case } 2 \\
\cdot \text { ULSD } \\
\text {-Sequential investment (step 1) } \\
\cdot \text { Some newer technologies }\end{array}$} \\
\hline & $\begin{array}{l}\text { On- } \\
\text { road }\end{array}$ & $\begin{array}{l}\text { Rail- } \\
\text { road }\end{array}$ & $\begin{array}{l}\text { Other } \\
\text { Off- } \\
\text { road }\end{array}$ & $\begin{array}{l}\text { Heating } \\
\text { Oil }\end{array}$ & $\begin{array}{l}\text { On- } \\
\text { road }\end{array}$ & $\begin{array}{l}\text { Rail- } \\
\text { road }\end{array}$ & $\begin{array}{l}\text { Other } \\
\text { Off- } \\
\text { road }\end{array}$ & $\begin{array}{l}\text { Heating } \\
\text { Oil }\end{array}$ \\
\hline Volume, MBD & 864 & 17 & 218 & 24 & 860 & 17 & 218 & 24 \\
\hline API Gravity, ${ }^{\circ} \mathrm{API}$ & 39.3 & 34.6 & 33.9 & 33.0 & 38.3 & 34.6 & 33.9 & 33.0 \\
\hline Specific gravity & .8286 & & & & .8334 & & & \\
\hline Sulfur, ppm & 8 & 220 & 350 & 260 & 8 & 330 & 280 & 260 \\
\hline Cetane Index & 53.8 & & & & 52.4 & & & \\
\hline Cetane Number, clear & 51.8 & & & & 50.4 & & & \\
\hline $\begin{array}{l}\text { Cetane Improver, } \\
\text { vol } \%\end{array}$ & .115 & & & & .000 & & & \\
\hline $\begin{array}{l}\text { Cetane Improver, } \\
\text { number increase }\end{array}$ & 7.1 & & & & 0.0 & & & \\
\hline Cetane Number, total & 58.8 & & & & 50.4 & & & \\
\hline Aromatics, vol $\%$ & 12.5 & 25.8 & 29.5 & 30.7 & 16.4 & 31.8 & 30.7 & 27.2 \\
\hline Viscosity, cSt & 2.78 & & & & 2.80 & & & \\
\hline ASTM IBP, ${ }^{\circ} \mathrm{F}^{\mathrm{a}}$ & 375 & & & & 376 & & & \\
\hline $\mathrm{T} 10$ & 434 & 428 & 441 & 420 & 435 & 423 & 441 & 438 \\
\hline $\mathrm{T} 30$ & 463 & 451 & 473 & 474 & 463 & 452 & 470 & 466 \\
\hline $\mathrm{T} 50$ & 492 & 487 & 508 & 506 & 493 & 489 & 505 & 499 \\
\hline $\mathrm{T} 70$ & 539 & 548 & 554 & 538 & 540 & 534 & 549 & 538 \\
\hline $\mathrm{T} 90$ & 591 & 605 & 610 & 597 & 591 & 585 & 595 & 588 \\
\hline $\mathrm{FBP}^{\mathrm{a}}$ & 635 & & & & 635 & & & \\
\hline NOx change, $\%^{\mathrm{b}}$ & -8.3 & & & & -5.9 & & & \\
\hline PM change, $\%{ }^{\mathrm{b}}$ & -19.8 & & & & -15.7 & & & \\
\hline \multicolumn{9}{|c|}{$\begin{array}{l}{ }^{\mathrm{a}} \text { IBP and FBP are based on survey reports of differences relative to T10 and T90 (NIPER 1994). } \\
{ }^{\mathrm{b}} \text { Relative to commercial fuel (Table } 4.1 \text { of Part II), with adjustment for future fleet change (see II- } \\
5.1 \text {. }\end{array}$} \\
\hline
\end{tabular}




\begin{tabular}{|c|c|c|c|c|c|c|c|c|}
\hline \multicolumn{9}{|c|}{ Table 6.3 (Continued). Properties of diesel fuels in PADD II summer 2010} \\
\hline \multirow[t]{2}{*}{ Property } & \multicolumn{4}{|c|}{$\begin{array}{l}\text { Case } 2.1 \\
\text {-ULSD } \\
\cdot \text { Reformulated for vehicle } \\
\text { performance } \\
\text { • Sequential investment (step 2) }\end{array}$} & \multicolumn{4}{|c|}{$\begin{array}{l}\text { Case } 2.2 \\
\text {-ULSD } \\
\text { - Reformulated for emissions } \\
\text { reduction } \\
\text { - Sequential investment (step 2) }\end{array}$} \\
\hline & $\begin{array}{l}\text { On- } \\
\text { road }\end{array}$ & $\begin{array}{l}\text { Rail- } \\
\text { road }\end{array}$ & $\begin{array}{l}\text { Other } \\
\text { Off- } \\
\text { road }\end{array}$ & $\begin{array}{l}\text { Heating } \\
\text { Oil }\end{array}$ & $\begin{array}{l}\text { On- } \\
\text { road }\end{array}$ & $\begin{array}{l}\text { Rail- } \\
\text { road }\end{array}$ & $\begin{array}{l}\text { Other } \\
\text { Off- } \\
\text { road }\end{array}$ & $\begin{array}{l}\text { Heating } \\
\text { Oil }\end{array}$ \\
\hline Volume, MBD & 863 & 17 & 218 & 24 & 863 & 17 & 218 & 24 \\
\hline API Gravity, ${ }^{\circ} \mathrm{API}$ & 39.1 & 34.6 & 33.9 & 33.0 & 39.1 & 34.6 & 33.9 & 33.0 \\
\hline Specific gravity & .8292 & & & & .8292 & & & \\
\hline Sulfur, ppm & 8 & 240 & 310 & 350 & 8 & 240 & 310 & 350 \\
\hline Cetane Index & 53.9 & & & & 53.9 & & & \\
\hline Cetane Number, clear & 51.9 & & & & 51.9 & & & \\
\hline $\begin{array}{l}\text { Cetane Improver, } \\
\text { vol } \%\end{array}$ & .108 & & & & .108 & & & \\
\hline $\begin{array}{l}\text { Cetane Improver, } \\
\text { number increase }\end{array}$ & 6.9 & & & & 6.9 & & & \\
\hline Cetane Number, total & 58.8 & & & & 58.8 & & & \\
\hline Aromatics, vol $\%$ & 12.5 & 31.6 & 28.7 & 32.8 & 12.5 & 31.6 & 28.7 & 32.8 \\
\hline Viscosity, cSt & 2.74 & & & & 2.74 & & & \\
\hline ASTM IBP, ${ }^{\circ} \mathrm{F}^{\mathrm{a}}$ & 375 & & & & 375 & & & \\
\hline $\mathrm{T} 10$ & 434 & 426 & 441 & 430 & 434 & 426 & 441 & 430 \\
\hline $\mathrm{T} 30$ & 464 & 451 & 471 & 475 & 464 & 451 & 471 & 475 \\
\hline $\mathrm{T} 50$ & 492 & 487 & 504 & 509 & 492 & 487 & 504 & 509 \\
\hline $\mathrm{T} 70$ & 540 & 543 & 546 & 542 & 540 & 543 & 546 & 542 \\
\hline T90 & 591 & 585 & 596 & 587 & 591 & 585 & 596 & 587 \\
\hline $\mathrm{FBP}^{\mathrm{a}}$ & 635 & & & & 635 & & & \\
\hline NOx change, $\%^{\mathrm{b}}$ & -8.2 & & & & -8.2 & & & \\
\hline PM change, $\%{ }^{\mathrm{b}}$ & -19.8 & & & & -19.8 & & & \\
\hline
\end{tabular}




\begin{tabular}{|c|c|c|c|c|c|c|}
\hline \multicolumn{7}{|c|}{$\begin{array}{l}\text { Table 6.4. Properties of on-road diesel fuels, by desulfurization (DeS) technology, in } \\
\text { PADD II summer } 2010\end{array}$} \\
\hline \multirow[t]{3}{*}{ Property } & \multicolumn{2}{|c|}{$\begin{array}{l}\text { Base Case } 2 \\
\cdot \text { ULSD } \\
\cdot \text { Sequential investment } \\
\text { (step 1) } \\
\cdot \text { Some newer } \\
\text { technologies } \\
\end{array}$} & \multicolumn{2}{|c|}{\begin{tabular}{|l} 
Case 2.1 \\
-ULSD \\
$\cdot$ Reformulated for \\
vehicle performance \\
•Sequential investment \\
(step 2) \\
\end{tabular}} & \multicolumn{2}{|c|}{\begin{tabular}{|l} 
Case 2.2 \\
$\cdot$ ULSD \\
$\cdot$ Reformulated for \\
emissions reduction \\
$\cdot$ Sequential investment \\
(step 2)
\end{tabular}} \\
\hline & \multicolumn{2}{|c|}{ DeS technology } & \multicolumn{2}{|c|}{ DeS technology } & \multicolumn{2}{|c|}{ DeS technology } \\
\hline & 2-stage & Advanced & 2-stage & Advanced & 2-stage & Advanced \\
\hline Volume, MBD & 772 & 88 & 775 & 98 & 775 & 98 \\
\hline API Gravity, ${ }^{\circ} \mathrm{API}$ & 38.7 & 34.3 & 39.3 & 38.1 & 39.3 & 38.1 \\
\hline Specific gravity & .8312 & .8535 & .8287 & .8341 & .8287 & .8341 \\
\hline Sulfur, ppm & 8 & 8 & 8 & 8 & 8 & 8 \\
\hline Cetane Index & 52.9 & 44.9 & 54.0 & 53.7 & 54.0 & 53.7 \\
\hline Cetane Number, clear & 50.9 & 42.9 & 52.0 & 51.7 & 52.0 & 51.7 \\
\hline $\begin{array}{l}\text { Cetane Improver, } \\
\text { vol\% }\end{array}$ & .000 & .000 & .108 & .117 & .108 & .117 \\
\hline $\begin{array}{l}\text { Cetane Improver, } \\
\text { number increase }\end{array}$ & 0.0 & 0.0 & 6.8 & 7.1 & 6.8 & 7.1 \\
\hline Cetane Number, total & 50.9 & 42.9 & 58.8 & 58.8 & 58.8 & 58.8 \\
\hline Aromatics, vol $\%$ & 14.9 & 29.7 & 12.5 & 12.5 & 12.5 & 12.5 \\
\hline Viscosity, cSt & 2.80 & 2.76 & 2.76 & 2.6 & 2.76 & 2.6 \\
\hline ASTM IBP,${ }^{\circ} F^{a}$ & 376 & 373 & 376 & 373 & 376 & 373 \\
\hline $\mathrm{T} 10$ & 435 & 432 & 435 & 432 & 435 & 432 \\
\hline $\mathrm{T} 30$ & 463 & 466 & 463 & 468 & 463 & 468 \\
\hline $\mathrm{T} 50$ & 492 & 495 & 491 & 504 & 491 & 504 \\
\hline $\mathrm{T} 70$ & 539 & 547 & 539 & 545 & 539 & 545 \\
\hline $\mathrm{T} 90$ & 591 & 590 & 591 & 590 & 591 & 590 \\
\hline $\mathrm{FBP}^{\mathrm{a}}$ & 635 & 634 & 635 & 634 & 635 & 634 \\
\hline NOx change, $\%^{\mathrm{b}}$ & -6.4 & -1.4 & -8.3 & -8.0 & -8.3 & -8.0 \\
\hline PM change, $\%^{\mathrm{b}}$ & -16.7 & -2.3 & -19.7 & -19.5 & -19.7 & -19.5 \\
\hline
\end{tabular}




\begin{tabular}{|c|c|c|c|c|c|}
\hline \multicolumn{6}{|c|}{$\begin{array}{l}\text { Table 6.5. Blendstocks of diesel fuels in PADD II summer } 2010 \\
\text { (Percent) }\end{array}$} \\
\hline \multirow[t]{2}{*}{$\begin{array}{l}\text { Blendstock } \\
\text { category }^{\mathrm{a}}\end{array}$} & \multicolumn{5}{|c|}{$\begin{array}{l}\text { Base Case } 1 \\
\cdot \text {-Low sulfur on-road diesel } \\
\text {-Investment as required }\end{array}$} \\
\hline & On-road & $\begin{array}{l}\text { Rail- } \\
\text { road }\end{array}$ & $\begin{array}{l}\text { Other } \\
\text { Off- } \\
\text { road }\end{array}$ & $\begin{array}{l}\text { Heating } \\
\text { Oil }\end{array}$ & Pool \\
\hline \multicolumn{6}{|l|}{ STRAIGHT-RUN: } \\
\hline Kerosene $(325-500)$ & & & & 14.3 & 0.3 \\
\hline Distillate (500-650) & 5.9 & 9.7 & 6.0 & 44.0 & 6.8 \\
\hline \multicolumn{6}{|l|}{$\begin{array}{l}\text { NON-CRACKED, } \\
\text { HYDROTREATED: }\end{array}$} \\
\hline Kerosene $(325-500)$ & 39.7 & 14.3 & 32.6 & 39.4 & 37.9 \\
\hline Distillate (500-650) & 29.4 & 25.7 & 36.8 & 2.4 & 30.2 \\
\hline \multicolumn{6}{|l|}{$\begin{array}{l}\text { CRACKED, } \\
\text { HYDROTREATED: }\end{array}$} \\
\hline Light Cycle Oil & 24.9 & 30.0 & 23.7 & & 24.2 \\
\hline \multicolumn{6}{|l|}{ HYDROCRACKED: } \\
\hline $\begin{array}{l}\text { Distillate (295- } \\
525)\end{array}$ & 0.1 & 20.4 & 0.9 & & 0.6 \\
\hline $\begin{array}{l}\text { CETANE } \\
\text { IMPROVER }\end{array}$ & & & & & \\
\hline $\begin{array}{l}{ }^{a} \text { True boiling point ra } \\
\text { name. }\end{array}$ & $\left.{ }^{\circ} \mathrm{F}\right)$ are & ted & len & catę & \\
\hline
\end{tabular}




\begin{tabular}{|c|c|c|c|c|c|c|}
\hline \multicolumn{7}{|c|}{$\begin{array}{c}\text { Table } 6.5 \text { (Continued). Blendstocks of diesel fuels in PADD II summer } 2010 \\
\text { (Percent) }\end{array}$} \\
\hline \multirow[t]{2}{*}{$\begin{array}{l}\text { Blendstock } \\
\text { category }^{\mathrm{a}}\end{array}$} & \multicolumn{6}{|c|}{$\begin{array}{l}\text { Case } 1.1 \\
\text {-ULSD } \\
\text { - Reformulated for vehicle performance } \\
\text {-Parallel investment }\end{array}$} \\
\hline & $\begin{array}{l}\text { On-road } \\
\text { from } \\
\text { 2-stage }\end{array}$ & $\begin{array}{l}\text { On-road from } \\
\text { advanced } \\
\text { DeS }\end{array}$ & $\begin{array}{l}\text { Rail- } \\
\text { road }\end{array}$ & $\begin{array}{l}\text { Other } \\
\text { Off- } \\
\text { road }\end{array}$ & $\begin{array}{l}\text { Heating } \\
\text { Oil }\end{array}$ & Pool \\
\hline \multicolumn{7}{|l|}{ STRAIGHT-RUN: } \\
\hline \multicolumn{7}{|l|}{ Kerosene $(325-500)$} \\
\hline \multicolumn{7}{|l|}{ Distillate (500-650) } \\
\hline \multicolumn{7}{|l|}{$\begin{array}{l}\text { NON-CRACKED, } \\
\text { HYDROTREATED: }\end{array}$} \\
\hline Kerosene (325-500) & 45.7 & & 14.3 & 25.4 & 34.4 & 41.0 \\
\hline Distillate (500-650) & 39.4 & & 35.4 & 40.0 & 35.6 & 39.4 \\
\hline \multicolumn{7}{|l|}{$\begin{array}{l}\text { CRACKED, } \\
\text { HYDROTREATED: }\end{array}$} \\
\hline Light Cycle Oil & 14.7 & & 30.0 & 30.0 & 30.0 & 18.2 \\
\hline \multicolumn{7}{|l|}{ HYDROCRACKED: } \\
\hline $\begin{array}{l}\text { Distillate (295- } \\
525)\end{array}$ & & & 20.4 & 4.6 & & 1.2 \\
\hline $\begin{array}{l}\text { CETANE } \\
\text { IMPROVER }\end{array}$ & 0.12 & & & & & 0.09 \\
\hline
\end{tabular}




\begin{tabular}{|c|c|c|c|c|c|c|}
\hline \multicolumn{7}{|c|}{$\begin{array}{c}\text { Table } 6.5 \text { (Continued). Blendstocks of diesel fuels in PADD II summer } 2010 \\
\text { (Percent) }\end{array}$} \\
\hline \multirow[t]{2}{*}{$\begin{array}{l}\text { Blendstock } \\
\text { category }^{\mathrm{a}}\end{array}$} & \multicolumn{6}{|c|}{$\begin{array}{l}\text { Case } 1.2 \\
\text {-ULSD } \\
\text { - Reformulated for emissions reduction } \\
\text { - Parallel investment }\end{array}$} \\
\hline & $\begin{array}{l}\text { On-road } \\
\text { from } \\
\text { 2-stage }\end{array}$ & $\begin{array}{l}\text { On-road from } \\
\text { advanced } \\
\text { DeS }\end{array}$ & $\begin{array}{l}\text { Rail- } \\
\text { road }\end{array}$ & $\begin{array}{l}\text { Other } \\
\text { Off- } \\
\text { road }\end{array}$ & $\begin{array}{l}\text { Heating } \\
\text { Oil }\end{array}$ & Pool \\
\hline \multicolumn{7}{|l|}{ STRAIGHT-RUN: } \\
\hline \multicolumn{7}{|l|}{ Kerosene $(325-500)$} \\
\hline \multicolumn{7}{|l|}{ Distillate (500-650) } \\
\hline \multicolumn{7}{|l|}{$\begin{array}{l}\text { NON-CRACKED, } \\
\text { HYDROTREATED: }\end{array}$} \\
\hline Kerosene (325-500) & 45.7 & & 36.9 & 27.4 & & 41.1 \\
\hline Distillate (500-650) & 39.4 & & 28.9 & 40.0 & 44.1 & 39.4 \\
\hline \multicolumn{7}{|l|}{$\begin{array}{l}\text { CRACKED, } \\
\text { HYDROTREATED: }\end{array}$} \\
\hline Light Cycle Oil & 14.7 & & 30.0 & 30.0 & 30.0 & 18.2 \\
\hline \multicolumn{7}{|l|}{ HYDROCRACKED: } \\
\hline $\begin{array}{l}\text { Distillate (295- } \\
525)\end{array}$ & & & 4.2 & 3.1 & 25.9 & 1.2 \\
\hline $\begin{array}{l}\text { CETANE } \\
\text { IMPROVER }\end{array}$ & 0.12 & & & & & 0.09 \\
\hline
\end{tabular}




\begin{tabular}{|c|c|c|c|c|c|c|}
\hline \multicolumn{7}{|c|}{$\begin{array}{c}\text { Table } 6.5 \text { (Continued). Blendstocks of diesel fuels in PADD II summer } 2010 \\
\text { (Percent) }\end{array}$} \\
\hline \multirow[t]{2}{*}{$\begin{array}{l}\text { Blendstock } \\
\text { category }^{\mathrm{a}}\end{array}$} & \multicolumn{6}{|c|}{$\begin{array}{l}\text { Base Case } 2 \\
\text {-ULSD } \\
\text {-Sequential investment (step 1) } \\
\text {-Some newer technologies }\end{array}$} \\
\hline & $\begin{array}{l}\text { On-road } \\
\text { from } \\
\text { 2-stage } \\
\end{array}$ & $\begin{array}{c}\text { On-road from } \\
\text { advanced } \\
\text { DeS } \\
\end{array}$ & $\begin{array}{l}\text { Rail- } \\
\text { road }\end{array}$ & $\begin{array}{l}\text { Other } \\
\text { Off- } \\
\text { road }\end{array}$ & $\begin{array}{c}\text { Heating } \\
\text { Oil }\end{array}$ & Pool \\
\hline \multicolumn{7}{|l|}{ STRAIGHT-RUN: } \\
\hline \multicolumn{7}{|l|}{ Kerosene $(325-500)$} \\
\hline \multicolumn{7}{|l|}{ Distillate (500-650) } \\
\hline \multicolumn{7}{|l|}{$\begin{array}{l}\text { NON-CRACKED, } \\
\text { HYDROTREATED: }\end{array}$} \\
\hline Kerosene (325-500) & 43.7 & 1.4 & 22.1 & 31.6 & 32.7 & 37.5 \\
\hline Distillate (500-650) & 39.9 & 62.3 & 31.7 & 37.6 & 37.3 & 41.0 \\
\hline \multicolumn{7}{|l|}{$\begin{array}{l}\text { CRACKED, } \\
\text { HYDROTREATED: }\end{array}$} \\
\hline Light Cycle Oil & 16.4 & 30.0 & 30.0 & 30.0 & 30.0 & 20.6 \\
\hline \multicolumn{7}{|l|}{ HYDROCRACKED: } \\
\hline $\begin{array}{l}\text { Distillate (295- } \\
525)\end{array}$ & & 6.4 & 16.1 & 0.8 & & 0.9 \\
\hline $\begin{array}{l}\text { CETANE } \\
\text { IMPROVER }\end{array}$ & & & & & & \\
\hline${ }^{\mathrm{a}}$ True boiling point ra & $\left({ }^{\circ} \mathrm{F}\right)$ are $\mathrm{i}$ & ated with ble & tock & ry n & & \\
\hline
\end{tabular}




\begin{tabular}{|c|c|c|c|c|c|c|}
\hline \multicolumn{7}{|c|}{$\begin{array}{c}\text { Table } 6.5 \text { (Continued). Blendstocks of diesel fuels in PADD II summer } 2010 \\
\text { (Percent) }\end{array}$} \\
\hline \multirow[t]{2}{*}{$\begin{array}{l}\text { Blendstock } \\
\text { category }^{\mathrm{a}}\end{array}$} & \multicolumn{6}{|c|}{$\begin{array}{l}\text { Case } 2.1 \\
\text {-ULSD } \\
\text {-Reformulated for vehicle performance } \\
\text {-Sequential investment (step 2) } \\
\end{array}$} \\
\hline & $\begin{array}{l}\text { On-road } \\
\text { from } \\
\text { 2-stage } \\
\end{array}$ & $\begin{array}{c}\text { On-road from } \\
\text { advanced } \\
\text { DeS } \\
\end{array}$ & $\begin{array}{l}\text { Rail- } \\
\text { road }\end{array}$ & $\begin{array}{l}\text { Other } \\
\text { Off- } \\
\text { road }\end{array}$ & $\begin{array}{c}\text { Heating } \\
\text { Oil }\end{array}$ & Pool \\
\hline \multicolumn{7}{|l|}{ STRAIGHT-RUN: } \\
\hline \multicolumn{7}{|l|}{ Kerosene $(325-500)$} \\
\hline \multicolumn{7}{|l|}{ Distillate (500-650) } \\
\hline \multicolumn{7}{|l|}{$\begin{array}{l}\text { NON-CRACKED, } \\
\text { HYDROTREATED: }\end{array}$} \\
\hline Kerosene $(325-500)$ & 45.2 & 34.4 & 34.3 & 31.8 & 9.3 & 40.8 \\
\hline Distillate (500-650) & 37.3 & 53.1 & 26.9 & 38.2 & 44.0 & 38.7 \\
\hline \multicolumn{7}{|l|}{$\begin{array}{l}\text { CRACKED, } \\
\text { HYDROTREATED: }\end{array}$} \\
\hline Light Cycle Oil & 17.4 & 4.1 & 30.0 & 30.0 & 30.0 & 19.3 \\
\hline \multicolumn{7}{|l|}{ HYDROCRACKED: } \\
\hline $\begin{array}{l}\text { Distillate (295- } \\
525)\end{array}$ & & & 8.9 & & 16.8 & 1.1 \\
\hline $\begin{array}{l}\text { CETANE } \\
\text { IMPROVER }\end{array}$ & 0.11 & 0.12 & & & & 0.08 \\
\hline
\end{tabular}




\begin{tabular}{|c|c|c|c|c|c|c|}
\hline \multicolumn{7}{|c|}{$\begin{array}{c}\text { Table } 6.5 \text { (Continued). Blendstocks of diesel fuels in PADD II summer } 2010 \\
\text { (Percent) }\end{array}$} \\
\hline \multirow[t]{2}{*}{$\begin{array}{l}\text { Blendstock } \\
\text { category }^{\mathrm{a}}\end{array}$} & \multicolumn{6}{|c|}{$\begin{array}{l}\text { Case } 2.2 \\
\text {-ULSD } \\
\text { - Reformulated for emissions reduction } \\
\text { - Sequential investment (step 2) }\end{array}$} \\
\hline & $\begin{array}{l}\text { On-road } \\
\text { from } \\
\text { 2-stage } \\
\end{array}$ & $\begin{array}{c}\text { On-road from } \\
\text { advanced } \\
\text { DeS }\end{array}$ & $\begin{array}{l}\text { Rail- } \\
\text { road }\end{array}$ & $\begin{array}{l}\text { Other } \\
\text { Off- } \\
\text { road }\end{array}$ & $\begin{array}{c}\text { Heating } \\
\text { Oil }\end{array}$ & Pool \\
\hline \multicolumn{7}{|l|}{ STRAIGHT-RUN: } \\
\hline \multicolumn{7}{|l|}{ Kerosene $(325-500)$} \\
\hline \multicolumn{7}{|l|}{ Distillate (500-650) } \\
\hline \multicolumn{7}{|l|}{$\begin{array}{l}\text { NON-CRACKED, } \\
\text { HYDROTREATED: }\end{array}$} \\
\hline Kerosene (325-500) & 45.2 & 34.4 & 34.3 & 31.8 & 9.3 & 40.8 \\
\hline Distillate $(500-650)$ & 37.3 & 53.1 & 26.9 & 38.2 & 44.0 & 38.7 \\
\hline \multicolumn{7}{|l|}{$\begin{array}{l}\text { CRACKED, } \\
\text { HYDROTREATED: }\end{array}$} \\
\hline Light Cycle Oil & 17.4 & 4.1 & 30.0 & 30.0 & 30.0 & 19.3 \\
\hline \multicolumn{7}{|l|}{ HYDROCRACKED: } \\
\hline $\begin{array}{l}\text { Distillate (295- } \\
525)\end{array}$ & & & 8.9 & & 16.8 & 1.1 \\
\hline $\begin{array}{l}\text { CETANE } \\
\text { IMPROVER }\end{array}$ & 0.11 & 0.12 & & & & 0.08 \\
\hline
\end{tabular}




\begin{tabular}{|c|c|c|c|c|c|c|}
\hline \multicolumn{7}{|c|}{ Table 6.6. Properties of gasolines in PADD II summer 2010} \\
\hline \multirow[t]{2}{*}{ Property } & \multicolumn{3}{|c|}{$\begin{array}{l}\text { Base Case } 1 \\
\text { - Low sulfur on-road diesel } \\
\text {-Investment as required }\end{array}$} & \multicolumn{3}{|c|}{$\begin{array}{l}\text { Case } 1.1 \\
\text {-ULSD } \\
\text { - Reformulated for vehicle } \\
\text { performance } \\
\text {-Parallel investment }\end{array}$} \\
\hline & CG & $\begin{array}{c}\mathrm{CG} / \\
\mathrm{EtOH} \\
\end{array}$ & RFG & CG & $\begin{array}{c}\mathrm{CG} / \\
\mathrm{EtOH} \\
\end{array}$ & RFG \\
\hline Volume, MBD & 1132 & 880 & 330.7 & 1132 & 880 & 330.7 \\
\hline Octane, $(\mathrm{R}+\mathrm{M}) / 2$ & 88.1 & 88.1 & 88.7 & 88.1 & 88.1 & 88.7 \\
\hline RVP, psi & 8.3 & 9.3 & 6.6 & 8.3 & 9.3 & 6.5 \\
\hline Aromatics, vol \% & 32.4 & 29.8 & 30.6 & 31.8 & 29.5 & 31.0 \\
\hline Benzene, vol \% & 1.68 & 2.44 & 0.90 & 1.74 & 2.41 & 0.90 \\
\hline Olefins, vol \% & 5.1 & 3.8 & 6.9 & 4.6 & 4.9 & 4.3 \\
\hline Sulfur, ppm & 30 & 30 & 30 & 30 & 30 & 30 \\
\hline E200, \% & 42.6 & 61.2 & 50.9 & 43.1 & 59.2 & 50.9 \\
\hline $\mathrm{E} 300, \%$ & 81.1 & 85.1 & 87.5 & 81.1 & 85.5 & 88.8 \\
\hline Oxygen, wt \% & 0.00 & 3.48 & 3.47 & 0.00 & 3.47 & 3.47 \\
\hline Specific gravity & .7546 & .7552 & .7581 & .7519 & .7573 & .7587 \\
\hline $\begin{array}{l}\text { Summer total TAP, } \\
\mathrm{mg} / \mathrm{mi}\end{array}$ & & & $63.1^{\mathrm{a}}$ & & & $63.1^{\mathrm{a}}$ \\
\hline $\begin{array}{l}\text { Summer exhaust TAP, } \\
\mathrm{mg} / \mathrm{mi}\end{array}$ & $73.4^{\mathrm{a}}$ & $73.5^{\mathrm{a}}$ & & $73.4^{\mathrm{a}}$ & $73.2^{\mathrm{a}}$ & \\
\hline NOx, mg/mi & 1176 & 1189 & 1172 & 1176 & 1186 & 1168 \\
\hline $\mathrm{VOC}, \mathrm{mg} / \mathrm{mi}$ & 1302 & 1432 & $993^{\mathrm{a}}$ & 1299 & 1429 & $994^{\mathrm{a}}$ \\
\hline
\end{tabular}




\begin{tabular}{|c|c|c|c|c|c|c|}
\hline \multicolumn{7}{|c|}{ Table 6.6 (Continued). Properties of gasolines in PADD II summer 2010} \\
\hline \multirow[t]{2}{*}{ Property } & \multicolumn{3}{|c|}{$\begin{array}{l}\text { Case } 1.2 \\
\text {-ULSD } \\
\text { - Reformulated for emissions } \\
\text { reduction } \\
\text {-Parallel investment } \\
\end{array}$} & \multicolumn{3}{|c|}{$\begin{array}{l}\text { Base Case } 2 \\
\text {-ULSD } \\
\text {-Sequential investment (step 1) } \\
\text {-Some newer technologies }\end{array}$} \\
\hline & CG & $\begin{array}{c}\mathrm{CG} / \\
\mathrm{EtOH} \\
\end{array}$ & RFG & CG & $\begin{array}{c}\mathrm{CG} / \\
\mathrm{EtOH} \\
\end{array}$ & RFG \\
\hline Volume, MBD & 1132 & 880 & 330.7 & 1132 & 880 & 330.7 \\
\hline Octane, $(\mathrm{R}+\mathrm{M}) / 2$ & 88.1 & 88.1 & 88.7 & 88.1 & 88.1 & 88.7 \\
\hline RVP, psi & 8.3 & 9.3 & 6.6 & 8.3 & 9.3 & 6.6 \\
\hline Aromatics, vol \% & 32.4 & 28.9 & 30.9 & 33.3 & 26.8 & 30.5 \\
\hline Benzene, vol \% & 1.70 & 2.50 & 0.90 & 1.62 & 2.63 & 0.90 \\
\hline Olefins, vol \% & 4.5 & 4.9 & 4.7 & 4.6 & 5.0 & 6.8 \\
\hline Sulfur, ppm & 30 & 30 & 30 & 30 & 30 & 30 \\
\hline $\mathrm{E} 200, \%$ & 43.0 & 61.7 & 50.9 & 42.9 & 60.9 & 51.0 \\
\hline $\mathrm{E} 300, \%$ & 80.7 & 85.3 & 88.8 & 80.8 & 85.4 & 88.0 \\
\hline Oxygen, wt \% & 0.00 & 3.47 & 3.47 & 0.00 & 3.49 & 3.47 \\
\hline Specific gravity & .7522 & .7569 & .7587 & .7530 & .7538 & .7587 \\
\hline $\begin{array}{l}\text { Summer total TAP, } \\
\mathrm{mg} / \mathrm{mi}\end{array}$ & & & $63.1^{\mathrm{a}}$ & & & $63.0^{\mathrm{a}}$ \\
\hline $\begin{array}{l}\text { Summer exhaust TAP, } \\
\mathrm{mg} / \mathrm{mi}\end{array}$ & $73.4^{\mathrm{a}}$ & $73.3^{\mathrm{a}}$ & & $73.2^{\mathrm{a}}$ & $73.3^{\mathrm{a}}$ & \\
\hline $\mathrm{NOx}, \mathrm{mg} / \mathrm{mi}$ & 1176 & 1188 & 1168 & 1177 & 1182 & 1172 \\
\hline $\mathrm{VOC}, \mathrm{mg} / \mathrm{mi}$ & 1305 & 1427 & 993 & 1307 & 1421 & $993^{\mathrm{a}}$ \\
\hline
\end{tabular}




\begin{tabular}{|c|c|c|c|c|c|c|}
\hline \multicolumn{7}{|c|}{ Table 6.6 (Continued). Properties of gasolines in PADD II summer 2010} \\
\hline \multirow[t]{2}{*}{ Property } & \multicolumn{3}{|c|}{$\begin{array}{l}\text { Case } 2.1 \\
\cdot \text { ULSD } \\
\cdot \text { Reformulated for vehicle } \\
\text { performance } \\
\text { •Sequential investment (step 2) }\end{array}$} & \multicolumn{3}{|c|}{$\begin{array}{l}\text { Case } 2.2 \\
\text {-ULSD } \\
\text { - Reformulated for emissions } \\
\text { reduction } \\
\text { • Sequential investment (step 2) }\end{array}$} \\
\hline & CG & $\begin{array}{c}\mathrm{CG} / \\
\mathrm{EtOH}\end{array}$ & RFG & CG & $\begin{array}{c}\mathrm{CG} / \\
\mathrm{EtOH}\end{array}$ & RFG \\
\hline Volume, MBD & 1132 & 880 & 330.7 & 1132 & 880 & 330.7 \\
\hline Octane, $(\mathrm{R}+\mathrm{M}) / 2$ & 88.1 & 88.1 & 88.7 & 88.1 & 88.1 & 88.7 \\
\hline RVP, psi & 8.3 & 9.3 & 6.5 & 8.3 & 9.3 & 6.5 \\
\hline Aromatics, vol \% & 31.1 & 29.7 & 31.1 & 31.1 & 29.7 & 31.1 \\
\hline Benzene, vol \% & 1.79 & 2.42 & 0.90 & 1.79 & 2.42 & 0.90 \\
\hline Olefins, vol \% & 4.0 & 4.7 & 3.8 & 4.0 & 4.7 & 3.8 \\
\hline Sulfur, ppm & 30 & 30 & 30 & 30 & 30 & 30 \\
\hline $\mathrm{E} 200, \%$ & 42.9 & 59.0 & 51.1 & 42.9 & 59.0 & 51.1 \\
\hline $\mathrm{E} 300, \%$ & 81.1 & 85.5 & 87.8 & 81.1 & 85.5 & 87.8 \\
\hline Oxygen, wt \% & 0.00 & 3.47 & 3.47 & 0.00 & 3.47 & 3.47 \\
\hline Specific gravity & .7497 & .7587 & .7587 & .7497 & .7587 & .7587 \\
\hline $\begin{array}{l}\text { Summer total TAP, } \\
\mathrm{mg} / \mathrm{mi}\end{array}$ & & & $63.2^{\mathrm{a}}$ & & & $63.2^{\mathrm{a}}$ \\
\hline $\begin{array}{l}\text { Summer exhaust TAP, } \\
\mathrm{mg} / \mathrm{mi}\end{array}$ & $73.3^{\mathrm{a}}$ & $73.5^{\mathrm{a}}$ & & $73.3^{\mathrm{a}}$ & $73.5^{\mathrm{a}}$ & \\
\hline $\mathrm{NOx}, \mathrm{mg} / \mathrm{mi}$ & 1174 & 1186 & 1169 & 1174 & 1186 & 1169 \\
\hline $\mathrm{VOC}, \mathrm{mg} / \mathrm{mi}$ & 1298 & 1431 & $994^{\mathrm{a}}$ & 1298 & 1431 & $994^{\mathrm{a}}$ \\
\hline
\end{tabular}




\begin{tabular}{|c|c|c|c|c|c|c|}
\hline \multicolumn{7}{|c|}{$\begin{array}{c}\text { Table 6.7. Blendstocks of gasolines in PADD II summer } 2010 \\
\text { (percent) }\end{array}$} \\
\hline \multirow[t]{2}{*}{ Property } & \multicolumn{3}{|c|}{$\begin{array}{l}\text { Base Case } 1 \\
\cdot \text { Low sulfur on-road diesel } \\
\cdot \text { Investment as required }\end{array}$} & \multicolumn{3}{|c|}{$\begin{array}{l}\text { Case } 1.1 \\
\text {-ULSD } \\
\text { - Reformulated for vehicle } \\
\text { performance } \\
\text {-Parallel investment }\end{array}$} \\
\hline & CG & $\begin{array}{c}\mathrm{CG} / \\
\mathrm{EtOH}\end{array}$ & RFG & $\mathrm{CG}$ & $\begin{array}{c}\mathrm{CG} / \\
\mathrm{EtOH}\end{array}$ & RFG \\
\hline $\mathrm{C} 4 \mathrm{~s}$ & 3.3 & 2.2 & 0.8 & 2.3 & 4.2 & 1.0 \\
\hline Reformate & 33.6 & 25.1 & 34.0 & 35.2 & 22.4 & 33.2 \\
\hline Straight run naphtha & 2.8 & 1.1 & 0.6 & 0.2 & 0.1 & 0.5 \\
\hline C5+ isomerate & 6.0 & 7.6 & 7.5 & 6.0 & 7.9 & 7.0 \\
\hline Iso-octane & & 0.6 & 0.7 & 0.7 & & \\
\hline FCC naphtha & 3.6 & 2.8 & 4.7 & 3.9 & 2.9 & 4.1 \\
\hline $\begin{array}{l}\text { Desulfurized FCC } \\
\text { naphtha }\end{array}$ & 35.6 & 36.5 & 14.5 & 33.0 & 37.9 & 12.6 \\
\hline \multicolumn{7}{|l|}{ Coker naphtha } \\
\hline Hydrocrackate & 2.1 & 5.5 & 23.9 & 7.3 & 6.7 & 30.0 \\
\hline Alkylate & 13.0 & 8.7 & 0.1 & 11.5 & 7.8 & \\
\hline Polymer gasolines & & & 3.1 & & & 1.7 \\
\hline Dimate & & & 0.1 & & & \\
\hline \multicolumn{7}{|l|}{ MTBE } \\
\hline Ethanol & & 10.0 & 10.0 & & 10.0 & 10.0 \\
\hline Natural gasoline & & & & & & \\
\hline
\end{tabular}




\begin{tabular}{|c|c|c|c|c|c|c|}
\hline \multicolumn{7}{|c|}{$\begin{array}{c}\text { Table } 6.7 \text { (Continued). Blendstocks of gasolines in PADD II summer } 2010 \\
\text { (percent) }\end{array}$} \\
\hline \multirow[t]{2}{*}{ Property } & \multicolumn{3}{|c|}{$\begin{array}{l}\text { Case } 1.2 \\
\cdot \text { ULSD } \\
\cdot \text { Reformulated for emissions } \\
\text { reduction } \\
\cdot \text { Parallel investment }\end{array}$} & \multicolumn{3}{|c|}{$\begin{array}{l}\text { Base Case } 2 \\
\text {-ULSD } \\
\text {-Sequential investment (step 1) } \\
\text {-Some newer technologies }\end{array}$} \\
\hline & $\mathrm{CG}$ & $\begin{array}{c}\mathrm{CG} / \\
\mathrm{EtOH}\end{array}$ & RFG & $\mathrm{CG}$ & $\begin{array}{c}\mathrm{CG} / \\
\mathrm{EtOH}\end{array}$ & RFG \\
\hline $\mathrm{C} 4 \mathrm{~s}$ & 2.7 & 3.9 & 1.0 & 3.2 & 2.8 & 0.8 \\
\hline Reformate & 31.9 & 26.5 & 33.7 & 31.8 & 22.3 & 37.5 \\
\hline Straight run naphtha & 0.2 & & 0.4 & & 4.1 & 1.1 \\
\hline $\mathrm{C} 5+$ isomerate & 7.7 & 5.8 & 6.7 & 9.1 & 3.6 & 7.7 \\
\hline Iso-octane & 0.7 & & & 0.7 & & \\
\hline FCC naphtha & 4.5 & 2.1 & 4.2 & 5.5 & 1.5 & 5.0 \\
\hline $\begin{array}{l}\text { Desulfurized FCC } \\
\text { naphtha }\end{array}$ & 33.6 & 40.0 & 12.6 & 32.3 & 38.7 & 11.5 \\
\hline \multicolumn{7}{|l|}{ Coker naphtha } \\
\hline Hydrocrackate & 9.8 & 0.9 & 29.4 & 7.8 & 4.2 & 23.3 \\
\hline Alkylate & 9.0 & 10.9 & & 9.6 & 12.9 & \\
\hline Polymer gasolines & & & 2.1 & & & 3.1 \\
\hline \multicolumn{7}{|l|}{ Dimate } \\
\hline \multicolumn{7}{|l|}{ MTBE } \\
\hline Ethanol & & 10.0 & 10.0 & & 10.0 & 10.0 \\
\hline Natural gasoline & & & & & & \\
\hline
\end{tabular}




\begin{tabular}{|c|c|c|c|c|c|c|}
\hline \multicolumn{7}{|c|}{$\begin{array}{c}\text { Table } 6.7 \text { (Continued). Blendstocks of gasolines in PADD II summer } 2010 \\
\text { (percent) }\end{array}$} \\
\hline \multirow[t]{2}{*}{ Property } & \multicolumn{3}{|c|}{$\begin{array}{l}\text { Case } 2.1 \\
\text {-ULSD } \\
\text { - Reformulated for vehicle } \\
\text { performance } \\
\text {-Sequential investment (step 2) }\end{array}$} & \multicolumn{3}{|c|}{$\begin{array}{l}\text { Case } 2.2 \\
\cdot \text { ULSD } \\
\cdot \text { Reformulated for emissions } \\
\text { reduction } \\
\cdot \text { Sequential investment (step 2) }\end{array}$} \\
\hline & $\mathrm{CG}$ & $\begin{array}{c}\mathrm{CG} / \\
\mathrm{EtOH}\end{array}$ & RFG & $\mathrm{CG}$ & $\begin{array}{c}\mathrm{CG} / \\
\mathrm{EtOH}\end{array}$ & RFG \\
\hline $\mathrm{C} 4 \mathrm{~s}$ & 3.0 & 3.2 & 0.9 & 3.0 & 3.2 & 0.9 \\
\hline Reformate & 31.4 & 26.1 & 38.7 & 31.4 & 26.1 & 38.7 \\
\hline Straight run naphtha & 0.1 & & 0.6 & 0.1 & & 0.6 \\
\hline C5+ isomerate & 7.9 & 5.7 & 10.7 & 7.9 & 5.7 & 10.7 \\
\hline Iso-octane & & 0.9 & & & 0.9 & \\
\hline FCC naphtha & 4.1 & 2.1 & 3.8 & 4.1 & 2.1 & 3.8 \\
\hline $\begin{array}{l}\text { Desulfurized FCC } \\
\text { naphtha }\end{array}$ & 34.2 & 36.5 & 9.8 & 34.2 & 36.5 & 9.8 \\
\hline \multicolumn{7}{|l|}{ Coker naphtha } \\
\hline Hydrocrackate & 4.9 & 10.2 & 24.5 & 4.9 & 10.2 & 24.5 \\
\hline Alkylate & 14.4 & 5.4 & & 14.4 & 5.4 & \\
\hline Polymer gasolines & & & 1.1 & & & 1.1 \\
\hline \multicolumn{7}{|l|}{ Dimate } \\
\hline \multicolumn{7}{|l|}{ MTBE } \\
\hline Ethanol & & 10.0 & 10.0 & & 10.0 & 10.0 \\
\hline Natural gasoline & & & & & & \\
\hline
\end{tabular}




\begin{tabular}{||l||c|c|c|c|c||}
\hline \multicolumn{7}{||c|}{ Table 6.8. Pooled gasoline blendstocks in PADD II summer 2010 } \\
(percent)
\end{tabular}




\begin{tabular}{|c|c|c|c|c|c|c|}
\hline \multicolumn{7}{|c|}{$\begin{array}{c}\text { Table 6.9. Refinery volume balance in PADD II summer } 2010 \\
\text { (Thousand barrels per day) }\end{array}$} \\
\hline \multirow[t]{2}{*}{ Property } & \multicolumn{6}{|c|}{ Case } \\
\hline & Base 1 & 1.1 & 1.2 & Base 2 & 2.1 & 2.2 \\
\hline \multicolumn{7}{|l|}{ Purchased inputs: } \\
\hline Crude oils & $4,385.1$ & $4,446.6$ & $4,446.6$ & $4,387.2$ & $4,452.7$ & $4,452.7$ \\
\hline Ethanol & 121.0 & 121.0 & 121.0 & 121.0 & 121.0 & 121.0 \\
\hline Methanol & 0.0 & 0.0 & 0.0 & 0.0 & 0.0 & 0.0 \\
\hline MTBE & 0.0 & 0.0 & 0.0 & 0.0 & 0.0 & 0.0 \\
\hline Other raw materials & 204.1 & 224.7 & 224.9 & 228.9 & 222.6 & 222.6 \\
\hline Total purchased inputs & $4,710.2$ & $4,792.3$ & $4,792.5$ & $4,737.1$ & $4,796.3$ & $4,796.3$ \\
\hline Total products & $4,813.9$ & $4,923.3$ & $4,923.7$ & $4,869.7$ & $4,926.0$ & $4,926.0$ \\
\hline
\end{tabular}




\begin{tabular}{|c|c|c|c|}
\hline \multicolumn{4}{|c|}{$\begin{array}{l}\text { Table 6.10. Hydrogen balance for PADD II refineries } \\
\text { Entry at top of cell is fuel oil equivalent barrels of hydrogen per day } \\
\text { (minus sign indicates consumption) } \\
\text { Entry at bottom of cell is process unit utilization } \\
\text { (calendar rate divided by stream day capacity x } 100 \text { percent) }\end{array}$} \\
\hline \multirow{2}{*}{ Process } & \multicolumn{3}{|c|}{ Case } \\
\hline & $\begin{array}{l}\text { Base } 1^{\text {a }} \\
\cdot \text { Low sulfur on-road } \\
\text { diesel } \\
\text {-Investment as required }\end{array}$ & $\begin{array}{l}1.1^{\text {a }} \\
\cdot \text { ULSD } \\
\cdot \text { Reformulated for } \\
\text { vehicle performance } \\
\cdot \text { Parallel investment }\end{array}$ & $\begin{array}{l}1.2^{\text {a }} \\
\cdot \text { ULSD } \\
\cdot \text { Reformulated for } \\
\text { emissions reduction } \\
\cdot \text { Parallel investment }\end{array}$ \\
\hline \multirow[t]{2}{*}{ Naphtha hydrotreating } & $-3,277$ & $-2,308$ & $-2,323$ \\
\hline & $(87.0)$ & $(83.1)$ & $(83.1)$ \\
\hline \multirow{2}{*}{$\begin{array}{l}\text { FCC gasoline } \\
\text { desulfurization }\end{array}$} & $-5,639$ & $-5,654$ & $-5,653$ \\
\hline & $(87.9)$ & $(87.9)$ & $(87.9)$ \\
\hline \multirow[t]{2}{*}{ Distillate desulfurization } & $-10,680$ & $-20,915$ & $-20,545$ \\
\hline & $(86.6)$ & $(175)$ & $(174)$ \\
\hline \multirow[t]{2}{*}{ Distillate dearomatization } & & $-2,309$ & $-2,759$ \\
\hline & & (New capacity) & (New capacity) \\
\hline \multirow[t]{2}{*}{ FCC feed hydrofining } & $-8,514$ & $-8,529$ & $-8,529$ \\
\hline & $(87.0)$ & $(87.0)$ & $(87.0)$ \\
\hline \multirow[t]{2}{*}{ Gas oil hydrocracking } & $-31,412$ & $-37,228$ & $-37,189$ \\
\hline & $(80.3)$ & $(97.4)$ & $(97.3)$ \\
\hline \multirow[t]{2}{*}{ Reforming } & $+38,186$ & $+37,796$ & $+37,845$ \\
\hline & $(79.8)$ & $(80.3)$ & $(80.4)$ \\
\hline \multirow[t]{2}{*}{$\mathrm{C} 4$ isomerization } & -44 & -44 & -44 \\
\hline & $(84.0)$ & $(84.0)$ & $(84.0)$ \\
\hline \multirow[t]{2}{*}{$\mathrm{C} 5 / \mathrm{C} 6$ isomerization } & -330 & -330 & -330 \\
\hline & $(84.0)$ & $(84.0)$ & $(84.0)$ \\
\hline \multirow[t]{2}{*}{ Iso-octane } & -316 & -316 & -316 \\
\hline & $(93.0)$ & $(93.0)$ & $(93.0)$ \\
\hline \multirow[t]{2}{*}{ Hydrogen production } & $+33,482$ & $+51,175$ & $+51,199$ \\
\hline & $(85.5)$ & $(129)$ & $(129)$ \\
\hline $\begin{array}{l}\text { Hydrogen to fuel and } \\
\text { losses }\end{array}$ & $-11,456$ & $-11,339$ & $-11,354$ \\
\hline
\end{tabular}




\begin{tabular}{|c|c|c|c|}
\hline \multicolumn{4}{|c|}{$\begin{array}{l}\text { Table } 6.10 \text { (Continued). Hydrogen balance for PADD II refineries } \\
\text { Entry at top of cell is fuel oil equivalent barrels of hydrogen per day } \\
\text { (minus sign indicates consumption) } \\
\text { Entry at bottom of cell is process unit utilization } \\
\text { (calendar rate divided by stream day capacity x } 100 \text { percent) }\end{array}$} \\
\hline \multirow{2}{*}{ Process } & \multicolumn{3}{|c|}{ Case } \\
\hline & $\begin{array}{l}\text { Base } 2^{\text {a }} \\
\cdot \text { ULSD } \\
\text {-Sequential investment } \\
\text { (step 1) } \\
\text {-Some newer } \\
\text { technologies } \\
\end{array}$ & $\begin{array}{l}2.1^{\mathrm{b}} \\
\cdot \text { ULSD } \\
\cdot \text { Reformulated for } \\
\text { vehicle performance } \\
\text {-Sequential investment } \\
\text { (step 2) }\end{array}$ & $\begin{array}{l}2.2^{\mathrm{b}} \\
\cdot \text { ULSD } \\
\cdot \text { Reformulated for } \\
\text { emissions reduction } \\
\cdot \text { Sequential investment } \\
\text { (step 2) }\end{array}$ \\
\hline \multirow[t]{2}{*}{ Naphtha hydrotreating } & $-3,409$ & $-2,795$ & $-2,795$ \\
\hline & $(84.4)$ & $(87.0)$ & $(87.0)$ \\
\hline \multirow{2}{*}{$\begin{array}{l}\text { FCC gasoline } \\
\text { desulfurization }\end{array}$} & $-5,420$ & $-5,681$ & $-5,681$ \\
\hline & $(87.9)$ & $(86.8)$ & $(86.8)$ \\
\hline \multirow[t]{2}{*}{ Distillate desulfurization } & $-21,666$ & $-20,026$ & $-20,026$ \\
\hline & $(185)$ & $(82.1)$ & $(82.1)$ \\
\hline \multirow[t]{2}{*}{ Distillate dearomatization } & & $-5,295$ & $-5,295$ \\
\hline & & (New capacity) & (New capacity) \\
\hline \multirow[t]{2}{*}{ FCC feed hydrofining } & $-8,484$ & $-8,421$ & $-8,421$ \\
\hline & $(87.0)$ & $(87.0)$ & $(87.0)$ \\
\hline \multirow[t]{2}{*}{ Gas oil hydrocracking } & $-30,743$ & $-36,212$ & $-36,212$ \\
\hline & $(81.1)$ & $(94.1)$ & $(94.1)$ \\
\hline \multirow[t]{2}{*}{ Reforming } & $+36,867$ & $+38,187$ & $+38,187$ \\
\hline & $(77.3)$ & $(81.2)$ & $(81.2)$ \\
\hline \multirow[t]{2}{*}{$\mathrm{C} 4$ isomerization } & -44 & -45 & -45 \\
\hline & $(84.0)$ & $(86.6)$ & $(86.6)$ \\
\hline \multirow[t]{2}{*}{$\mathrm{C} 5 / \mathrm{C} 6$ isomerization } & -330 & -360 & -360 \\
\hline & $(84.0)$ & $(91.6)$ & $(91.6)$ \\
\hline \multirow[t]{2}{*}{ Iso-octane } & -316 & -316 & -316 \\
\hline & $(93.0)$ & $(93.0)$ & $(93.0)$ \\
\hline \multirow[t]{2}{*}{ Hydrogen production } & $+44,605$ & $+52,422$ & $+52,422$ \\
\hline & $(113)$ & $(99.6)$ & $(99.6)$ \\
\hline $\begin{array}{l}\text { Hydrogen to fuel and } \\
\text { losses }\end{array}$ & $-11,060$ & $-11,456$ & $-11,456$ \\
\hline
\end{tabular}




\begin{tabular}{|c|c|c|c|c|c|c|}
\hline \multicolumn{7}{|c|}{$\begin{array}{l}\text { Table 6.11. Process capacity expansions and additions in PADD II summer } 2010 \\
\left(\text { MBSD) }{ }^{\mathrm{a}}\right.\end{array}$} \\
\hline \multirow[t]{2}{*}{ Process } & \multicolumn{6}{|c|}{ Case } \\
\hline & Base $1^{\mathrm{b}}$ & $1.1^{\mathrm{b}}$ & $1.2^{\mathrm{b}}$ & Base $2^{b}$ & $2.1^{\mathrm{c}}$ & $2.2^{\mathrm{c}}$ \\
\hline Crude distillation & & 64 & 64 & 2 & 68 & 68 \\
\hline Vacuum distillation & & 38 & 38 & 3 & 39 & 39 \\
\hline Delayed coking & & 18 & 18 & & 20 & 20 \\
\hline Gas oil hydrocracking & & 71 & 70 & & 56 & 56 \\
\hline $\begin{array}{l}\text { Distillate deep } \\
\text { hydrodesulfurization, 1st stage } \\
\text { (unit HD1) }\end{array}$ & & 529 & 527 & 536 & & \\
\hline $\begin{array}{l}\text { Distillate deep } \\
\text { hydrodesulfurization, 2nd } \\
\text { stage (unit HS2) }\end{array}$ & & 496 & 485 & 535 & & \\
\hline $\begin{array}{l}\text { Distillate deep } \\
\text { hydrodesulfurization and } \\
\text { dearomatization, 2nd stage } \\
\text { (unit HD2) }\end{array}$ & & 78 & 84 & & 31 & 31 \\
\hline $\begin{array}{l}\text { Revamp of distillate } \\
\text { desulfurization to HD1 + HS2 }\end{array}$ & & 211 & 211 & 211 & & \\
\hline Conversion of HS2 to HD2 & & & & & 132 & 132 \\
\hline $\begin{array}{l}\text { Advanced distillate } \\
\text { desulfurization technology }\end{array}$ & & & & 90 & & \\
\hline $\mathrm{C} 4$ isomerization & & & & & 1 & 1 \\
\hline $\mathrm{C} 5 / \mathrm{C} 6$ isomerization & & & & & 18 & 18 \\
\hline Naphtha splitter & & 30 & 26 & & 34 & 34 \\
\hline Hydrogen plant, FOE & & 21 & 21 & 13 & 9 & 9 \\
\hline Sulfur plant, tons per day & & 0.5 & 0.5 & & 0.4 & 0.4 \\
\hline \multicolumn{7}{|c|}{ 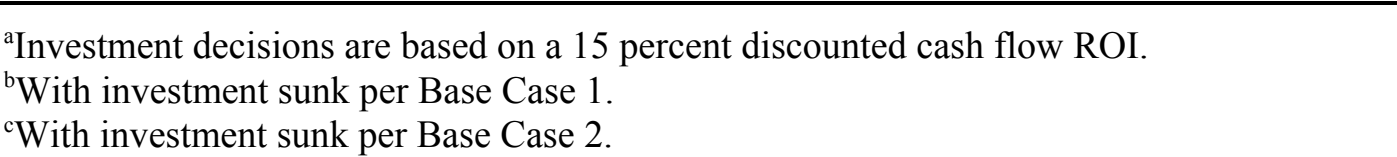 } \\
\hline
\end{tabular}




\begin{tabular}{|c|c|c|c|c|c|c|}
\hline \multicolumn{7}{|c|}{$\begin{array}{l}\text { Table 6.12. Cost of process capacity expansions and additions in PADD II summer } \\
\qquad 2010(\$ M M)^{\mathrm{a}}\end{array}$} \\
\hline \multirow[t]{2}{*}{ Process } & \multicolumn{6}{|c|}{ Case } \\
\hline & Base $1^{\mathrm{b}}$ & $1.1^{\mathrm{b}}$ & $1.2^{\mathrm{b}}$ & Base $2^{\mathrm{b}}$ & $2.1^{\mathrm{c}}$ & $2.2^{\mathrm{c}}$ \\
\hline Crude distillation & & 89 & 89 & 3 & 95 & 95 \\
\hline Vacuum distillation & & 50 & 50 & 4 & 50 & 50 \\
\hline Delayed coking & & 120 & 120 & & 135 & 135 \\
\hline Gas oil hydrocracking & & 867 & 862 & & 692 & 692 \\
\hline $\begin{array}{l}\text { Distillate deep } \\
\text { hydrodesulfurization, 1st stage } \\
\text { (unit HD1) }\end{array}$ & & 1,590 & 1,587 & 1,528 & & \\
\hline $\begin{array}{l}\text { Distillate deep } \\
\text { hydrodesulfurization, 2nd stage } \\
\text { (unit HS2) }\end{array}$ & & 461 & 451 & 489 & & \\
\hline $\begin{array}{l}\text { Distillate deep } \\
\text { hydrodesulfurization and } \\
\text { dearomatization, 2nd stage (unit } \\
\text { HD2) }\end{array}$ & & 189 & 205 & & 76 & 76 \\
\hline $\begin{array}{l}\text { Revamp of distillate } \\
\text { desulfurization to HD1 + HS2 }\end{array}$ & & 601 & 601 & 575 & & \\
\hline Conversion of HS2 to HD2 & & & & & 141 & 141 \\
\hline $\begin{array}{l}\text { Advanced distillate } \\
\text { desulfurization technology }\end{array}$ & & & & 202 & & \\
\hline $\mathrm{C} 4$ isomerization & & & & & 10 & 10 \\
\hline C5/C6 isomerization & & & & & 107 & 107 \\
\hline Naphtha splitter & & 20 & 18 & & 23 & 23 \\
\hline Hydrogen plant, FOE & & 759 & 760 & 477 & 335 & 335 \\
\hline Sulfur plant, tons per day & & 94 & 93 & & 78 & 78 \\
\hline $\begin{array}{l}\text { Land, buildings, catalyst, } \\
\text { chemical, spares, environmental, } \\
\text { other }\end{array}$ & & 542 & 541 & 367 & 215 & 215 \\
\hline Total & & 5,381 & 5,376 & 3,644 & 1,956 & 1,956 \\
\hline \multicolumn{7}{|c|}{ 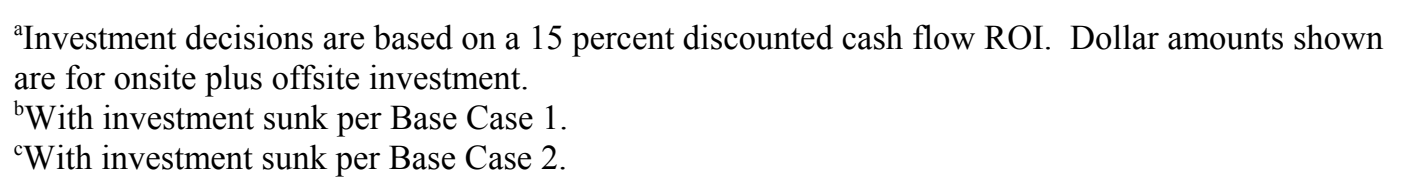 } \\
\hline
\end{tabular}




\begin{tabular}{|c|c|c|c|c|c|c|}
\hline \multicolumn{7}{|c|}{$\begin{array}{l}\text { Table 6.13. Components of refinery cost changes in PADD II summer } 2010 \\
\text { (cents per gallon of RFD) } \\
\text { All cost changes in table are relative to Base Case 1 }\end{array}$} \\
\hline \multirow[t]{2}{*}{ Process } & \multicolumn{6}{|c|}{ Case } \\
\hline & Base 1 & 1.1 & 1.2 & Base 2 & 2.1 & 2.2 \\
\hline $\begin{array}{l}\text { Raw material costs and product } \\
\text { revenue changes }\end{array}$ & 0.0 & -0.3 & -0.4 & -1.4 & -0.5 & -0.5 \\
\hline Processing costs & 0.0 & 0.9 & 0.9 & 0.9 & 1.1 & 1.1 \\
\hline Capital charges & 0.0 & 6.8 & 6.8 & 4.6 & $7.4^{\mathrm{a}}$ & $7.4^{\mathrm{a}}$ \\
\hline Fixed operating costs & 0.0 & 1.6 & 1.6 & 1.1 & $1.7^{\mathrm{a}}$ & $1.7^{\mathrm{a}}$ \\
\hline $\begin{array}{l}\text { Total cost change } \\
\text { (10 percent ROI) }\end{array}$ & 0.0 & 9.0 & 8.9 & 5.2 & 9.7 & 9.7 \\
\hline \multicolumn{7}{|c|}{$\begin{array}{l}\text { anvestment (including advanced technology desulfurization capacity) was sunk per Base Case } 2 \text {. } \\
\text { Compared to Base Case } 2 \text {, Cases } 2.1 \text { and } 2.2 \text { have lower utilization and lower cash margin for the } \\
\text { advanced technology desulfurization unit. The cash loss is represented by a post-optimal adjustment } \\
\text { to the objective function to cover the associated capital charges and fixed operating costs. It is } \\
\text { assumed that the advanced technology desulfurization unit operates at full utilization between } 2005 \\
\text { and 2010. Unit utilization drops substantially after 2010, per the refinery model solution for Cases } \\
2.1 \text { and 2.2. }\end{array}$} \\
\hline
\end{tabular}

\begin{tabular}{|c|c|}
\hline \multicolumn{2}{|c|}{$\begin{array}{l}\text { Table 6.14. Quality of crude oil processed in } \\
\text { PADD II summer } 2010\end{array}$} \\
\hline Property & All Cases \\
\hline Sulfur content, wt \% & 1.27 \\
\hline Gravity, ${ }^{\circ} \mathrm{API}$ & 34.0 \\
\hline
\end{tabular}




\begin{tabular}{|c|c|c|c|}
\hline \multicolumn{4}{|c|}{$\begin{array}{c}\text { Table 6.15. Refinery energy use changes in PADD II summ } \\
\text { Daily energy use changes relative to Base Case } 1\end{array}$} \\
\hline & $\begin{array}{c}\text { Purchased electricity, } \\
1000 \mathrm{Kwh}\end{array}$ & | & $\begin{array}{l}\text { Plant fuel, } \\
\text { FOE-MBD }\end{array}$ \\
\hline $\begin{array}{l}\text { Base Case } 1 \\
\text { - Low sulfur on-road diesel } \\
\text {-Investment as required } \\
\end{array}$ & 0 & I & 0 \\
\hline $\begin{array}{l}\text { Case } 1.1 \\
\text {-ULSD } \\
\text { - Reformulated for vehicle } \\
\text { performance } \\
\text {-Parallel investment } \\
\end{array}$ & 3,893 & 1 & 26 \\
\hline $\begin{array}{l}\text { Case } 1.2 \\
\cdot \text { ULSD } \\
\text { - Reformulated for emissions } \\
\text { reduction } \\
\text {-Parallel investment } \\
\end{array}$ & 3,883 & i & 26 \\
\hline $\begin{array}{l}\text { Base Case } 2 \\
\text {-ULSD } \\
\text { - Sequential investment (step 1) } \\
\text {-Some newer technologies } \\
\end{array}$ & 2,852 & I & 20 \\
\hline $\begin{array}{l}\text { Case } 2.1 \\
\cdot \text {-ULSD } \\
\text { - Reformulated for vehicle } \\
\text { performance } \\
\text { • Sequential investment (step 2) }\end{array}$ & 3,878 & I & 27 \\
\hline $\begin{array}{l}\text { Case } 2.2 \\
\text {-ULSD } \\
\text { - Reformulated for emissions } \\
\text { reduction } \\
\text { - Sequential investment (step 2) }\end{array}$ & 3,828 & I & 27 \\
\hline
\end{tabular}




\section{I-6.2 CASE 1.1: PARALLEL INVESTMENT AND VEHICLE PERFORMANCE RFD}

In Case 1.1, ULSD is reformulated for vehicle performance. Given early notice of diesel fuel quality requirements, refiners follow a parallel investment pathway. Key results for Case 1.1 and results of all other cases are summarized in Tables 6.2 through 6.15.

Due to the high cetane number specification for vehicle performance RFD, distillate upgrading is via twostage deep hydroprocessing (Table 6.11). There is no investment in advanced technologies that selectively remove sulfur (with low hydrogen consumption and without significant cetane improvement). Five existing distillate desulfurization units are revamped into two-stage units. For new two-stage installations, 14 percent of second-stage capacity is for units with 70 percent dearomatization capability. Lesser dearomatization occurs in the first- and second-stage distillate desulfurization units: 20 percent and 35 percent dearomatization, respectively.

Cetane improver is important in satisfying the on-road diesel cetane number specification. Table 6.3 shows that the cetane improver treat rate is 0.122 vol percent. This rate is substantially higher than current regional averages. For example, in the API/NPRA (1997) survey, the cetane improver treat rate for CARB diesel was 0.027 vol percent.

Table 6.2 shows that the average cost increase for RFD in Case 1.1 is 9.0 cents per gallon (cpg). The major component of this cost increase is capital charges (Table 6.13). Cetane improver (in the raw material category of Table 6.13) contributes $1.2 \mathrm{cpg}$ to the cost increase.

Tighter on-road specifications cause an increase in aromatics in the off-road diesel pool in Case 1.1. The offroad diesel pool has an aromatics content of $29.3 \mathrm{vol}$ percent, compared with 24.2 vol percent in Base Case 1. A comparison of off-road diesel blendstocks for the two cases (Table 6.5) shows a decline in straight-run blendstocks (higher sulfur content, lower aromatics content) and an increase in hydrotreated light-cycle oil (lower sulfur content, higher aromatics content) in Case 1.1 compared to Base Case 1. The decline in sulfur content for the off-road diesel pool (327 ppm compared to $350 \mathrm{ppm}$ for Base Case 1) is discussed in Section I-6.8.

RFD in Case 1.1 has much-improved emissions characteristics, relative to the commercial reference fuel. NOx emissions are reduced by 8.3 percent, and PM emissions are reduced by 19.8 percent (Table 6.3). Table 6.15 shows that diesel fuel reformulation requires a sizeable increase in refinery energy use. Changes in refinery energy and the associated pollutant emissions need to be included in a complete evaluation of the environmental impacts of RFD.

\section{I-6.3 CASE 1.2: PARALLEL INVESTMENT AND EMISSIONS REDUCTION RFD}

In Case 1.2, ULSD is reformulated for emissions reduction. Given early notice of diesel fuel quality requirements, refiners follow a parallel investment pathway. Key results for Case 1.2 and results of all other cases are summarized in Tables 6.3 through 6.15 .

Emissions specifications for Case 1.2 are based on Case 1.1, in which NOx is reduced by 8.3 percent, and PM is reduced by 19.8 percent. In both cases, virtually the same blendstocks are used for RFD (Table 6.5).

Because there is little blendstock flexibility to exploit in achieving the challenging emissions requirements, the eigenfuel-based emissions reduction specifications offer little refining economic benefit over the vehicle performance specifications, and refining impacts are about the same in both cases. 
In Case 1.2, distillate upgrading is via two-stage deep hydroprocessing (Table 6.11). There is no investment in advanced technologies that selectively remove sulfur. Five existing distillate desulfurization units are revamped into two-stage units. For new two-stage installations, 15 percent of second-stage capacity is for units with 70 percent dearomatization capability.

Table 6.3 shows that the cetane improver treat rate is 0.115 volume percent, slightly lower than with vehicle performance specifications, but still substantially higher than current regional averages.

Table 6.2 shows that the average cost increase for RFD in Case 1.1 is $8.9 \mathrm{cpg}$, slightly lower than in Case 1.1. The major component of this cost increase is capital charges (Table 6.13). Cetane improver (in the raw material category of Table 6.13) contributes $1.1 \mathrm{cpg}$ to the cost increase.

In Case 1.2, the off-road diesel pool has an aromatics content of 29.3 vol percent, compared with 24.2 vol percent in Base Case 1. A comparison of off-road diesel blendstocks for the two cases (Table 6.5) shows a decline in straight-run blendstocks (higher sulfur content, lower aromatics content) and an increase in hydrotreated light-cycle oil (lower sulfur content, higher aromatics content) in Case 1.2 compared to Base Case 1. The decline in sulfur content for the off-road diesel pool (333 ppm compared to $350 \mathrm{ppm}$ for Base Case 1) is discussed in Section I-6.8.

Table 6.15 shows that diesel fuel reformulation requires a sizeable increase in refinery energy use. Changes in refinery energy and the associated pollutant emissions need to be included in a complete evaluation of the impacts of RFD.

\section{I-6.4 BASE CASE 2: ULSD}

Base Case 2 is one of two base cases used as references to show comparative and cumulative impacts of different reformulation and investment pathways. Base Case 2 is the first step of a sequential investment pathway in which refiners do not have early notice of diesel fuel reformulation requirements. In this first step, ULSD must satisfy future requirements for sulfur content (15 ppm maximum) and cetane number (40 minimum), but there are no requirements for reformulation to satisfy vehicle performance or emissions reduction specifications. The second sequential step involves refiner investments for diesel fuel reformulation, given that investments for ULSD production have already been made in the first step.

Like Base Case 1, Base Case 2 is not representative of current quality requirements for off-road diesel fuels, which have premised sulfur specifications of $500 \mathrm{ppm}$ maximum. By using this off-road sulfur specification in all cases, we have intentionally removed a variable from the study. Key results for Base Case 2 and all other cases are summarized in Tables 6.2 through 6.15 .

We premise limited market penetration of advanced technologies that selectively remove sulfur, with low hydrogen consumption. Of the 27 PADD II refineries, 13 refineries currently have distillate hydrotreating, and we assume that these refineries produce low sulfur diesel fuel. Given the Baker \& O'Brien (2001) projection that thirty-eight percent of current low sulfur diesel producers will engage in revamps of distillate hydrotreating units, we allow revamping in up to five refineries. For the remaining 22 refineries, we arbitrarily assume that up to three refineries (i.e., almost 15 percent technology penetration) can invest in advanced desulfurization technologies.

In Base Case 2, five existing distillate desulfurization units are revamped into two-stage units (Table 6.11). Investment in advanced technologies lowers the cost of ULSD production. Relative to Base Case 1, the cost increase for ULSD is $5.2 \mathrm{cpg}$ (Table 6.2). Sensitivity runs show a ULSD cost increase of $7.9 \mathrm{cpg}$ via twostage deep hydrotreating, with no investment allowed in advanced technologies; and a cost increase of 3.7 
cpg with no constraints on investment in advanced technologies. These results have similarities with the "ULSD Supply and Cost" curve in Baker \& O'Brien (2001). For the purposes of illustration only, re-scaling the numbers in the Baker \& O'Brien curve to approximately match our study: $7.9 \mathrm{cpg}$ falls to $5.7 \mathrm{cpg}$ at 85 percent volume. With advanced technology costs at $3.7 \mathrm{cpg}:(5.7 \mathrm{cpg}) *(.85)+(3.7 \mathrm{cpg}) *(0.15)=5.4 \mathrm{cpg}$ for the overall average cost increase.

ULSD product quality is dramatically different for different desulfurization technologies. Table 6.4 shows that ULSD produced with advanced technologies has substantially lower cetane quality, higher aromatics content, and poorer emissions quality. While the advanced technologies are attractive for sulfur reduction, these technologies have little impact on those properties (e.g., cetane quality and aromatics content) which define "reformulation" in this study.

For new two-stage installations, all of the second-stage capacity is for units with deep desulfurization, with 35 percent dearomatization. With 20 percent dearomatization in the first-stage, dearomatization across the two stages explains a large part of the on-road diesel aromatics reduction (to 16.4 vol percent) relative to Base Case 1. There is also a shift of aromatics into the off-road diesel pool, relative to Base Case 1. In Base Case 2 , the off-road diesel pool has an aromatics content of 30.4 vol percent, compared with 24.2 vol percent in Base Case 1. A comparison of off-road diesel blendstocks for the two cases (Table 6.5) shows a decline in straight-run blendstocks (higher sulfur content, lower aromatics content) and an increase in hydrotreated lightcycle oil (lower sulfur content, higher aromatics content) in Base Case 2. The decline in sulfur content for the off-road diesel pool (281 ppm compared to 350 ppm for Base Case 1) is discussed in Section I-6.8.

Table 6.15 shows that ULSD production requires a sizeable increase in refinery energy use, relative to Base Case 1. Changes in refinery energy and the associated pollutant emissions need to be included in a complete evaluation of the impacts of ULSD.

\section{I-6.5 CASE 2.1: SEQUENTIAL INVESTMENT AND VEHICLE PERFORMANCE RFD}

Case 2.1 represents a second sequential step along an investment pathway in which refiners do not have early notice of diesel fuel reformulation requirements. Given that the investments for ULSD production have already been made in the first step, Case 2.1 is the second sequential step for diesel fuel reformulation to satisfy vehicle performance specifications. Key results for Case 2.1 and results of all other cases are summarized in Tables 6.2 through 6.15 .

In the Case 2.1 step to RFD with high cetane number and low aromatics, there is drop in utilization of advanced technologies that selectively remove sulfur. Refiners who invested in advanced technology capacity in step one of the investment pathway must either purchase high cetane/low aromatics stocks from those refineries with two-stage deep hydroprocessing capacity, or they must invest in distillate dearomatization capacity (in our premises, this subset of refineries does not have the option of changing the distillate product mix). Tables 6.11 and 6.12 show that gas oil hydrocracking, distillate dearomatization, and hydrogen production are among the investments made in Case 2.1. There is substantial conversion of second-stage hydrodesulfurization capacity to second stage dearomatization capacity. These investments are added to the Base Case 2 investments to derive total investments for the two-step sequential investment pathway.

Investment (including advanced technology desulfurization capacity) was sunk per Base Case 2. The sunk investment approach assumes that the advanced technology desulfurization unit operates at full utilization between 2005 and 2019. However, for Case 2.1, unit utilization drops substantially after 2010. With its lower utilization, the advanced technology desulfurization unit has a lower cash margin, which is contrary 
to the sunk investment assumptions. Objective function adjustments have been made to cover the associated capital charges and fixed operating costs.

Cetane improver is important in satisfying the on-road diesel cetane number specification. Table 6.3 shows that the cetane improver treat rate is 0.108 volume percent, which is substantially higher than current regional averages.

Table 6.2 shows that the average cost increase for RFD in Case 2.1 is $9.7 \mathrm{cpg}$. The major component of this cost increase is capital charges (Table 6.13). Cetane improver (in the raw material category of Table 6.13) contributes $1.0 \mathrm{cpg}$ to the cost increase. Compared to the early notice pathway of Case 1.1, capital investment increases in Case 2.1 (by 4.1 percent), and the per gallon cost increases by 0.7 cents.

In Case 2.1, the off-road diesel pool has an aromatics content of 29.1 vol percent, compared with 24.2 vol percent in Base Case 1. A comparison of off-road diesel blendstocks for the two cases (Table 6.5) shows a decline in straight-run blendstocks (higher sulfur content, lower aromatics content) and an increase in hydrotreated light-cycle oil (lower sulfur content, higher aromatics content) in Case 2.1. The decline in sulfur content for the off-road diesel pool (309 ppm compared to $350 \mathrm{ppm}$ for Base Case 1) is discussed in Section I-6.8.

RFD in Case 2.1 has much-improved emissions characteristics, relative to the commercial reference fuel. NOx emissions are reduced by 8.2 percent, and PM emissions are reduced by 19.8 percent (Table 6.3). Because cetane quality and aromatics content are controlled, emissions quality is about the same for RFD produced by different desulfurization technologies. However, cetane and aromatics control is more expensive for refiners using advanced desulfurization technologies. Compared with RFD produced with two-stage technologies, the marginal cost of RFD produced with advanced technologies is $1.7 \mathrm{cpg}$ higher.

Table 6.15 shows that diesel fuel reformulation requires a sizeable increase in refinery energy use. Changes in refinery energy and the associated pollutant emissions need to be included in a complete evaluation of the environmental impacts of RFD.

\section{I-6.6 CASE 2.2: SEQUENTIAL INVESTMENT AND EMISSIONS REDUCTION RFD}

Instead of using cetane number and aromatics specifications for RFD, Case 2.2 uses emissions specifications based on results of Case 2.1. NOx emissions are to be reduced by 8.2 percent, and PM emissions are to be reduced by 19.8 percent In spite of these specification changes, refinery operations and investments are the same for both Case 2.1 and Case 2.2. Thus, there are no refinery economic benefits from using the emissions reduction specifications versus the vehicle performance recipe specifications in these cases, which have very challenging specifications. As specifications become tighter, the processing routes and blendstock choices become more limited. The emissions reduction specifications thus have little refining flexibility to exploit, and refinery benefits vanish for the flexible emissions reduction specifications relative to the vehicle performance recipe specifications.

\section{I-6.7 EMISSIONS SPECIFICATION BENEFITS}

It has been assumed that the maximum vehicle performance specifications are cap specifications which require safety margins to assure that the specifications are not violated. Therefore, the 55 minimum cetane number for RFD is represented in ORNL-RYM ${ }_{2002}$ as a refinery target of 58.8 minimum cetane number, and the $15 \mathrm{vol}$ percent maximum aromatics content is represented as a refinery target of $12.5 \mathrm{vol}$ percent

maximum aromatics content. As discussed in prior Section I-6.6, there are no refinery economic benefits 
from using the emissions reduction specifications in Case 2.2 when the vehicle performance recipe specifications are at these very challenging levels, which limit processing and blendstock options.

Sensitivity cases have been examined to determine if there are emissions modeling benefits for refining with less challenging specifications in Cases 2.1 and 2.2. In these sensitivity cases, the vehicle performance specifications are assumed to be average specifications. For example, the $15 \mathrm{vol}$ percent maximum aromatics specification can be exceeded occasionally during the production season, as long as the seasonal average for the region is not greater than 15 vol percent. Therefore, the 55 minimum cetane number for RFD is represented in ORNL-RYM ${ }_{2002}$ as a refinery target of 55 minimum cetane number, and the 15 vol percent maximum aromatics content is represented as a refinery target of $15 \mathrm{vol}$ percent maximum aromatics content. As shown in Table 6.16, emissions reduction specifications for the sequential investment pathway in PADD II reduce RFD production costs by $0.2 \mathrm{cpg}$ and investment (over the total sequential pathway) by 6.4 percent, compared to vehicle performance specifications. Table 6.17 shows that, with the increased blending flexibility of emissions reduction specifications for RFD, there are increases in API gravity, cetane number and aromatics content, while the same emissions quality is achieved for both emissions reduction and vehicle performance RFDs.

Emissions reduction specifications would show increasing refinery economic benefits over vehicle performance recipe specifications in circumstances where increasing numbers of processing routes and blendstocks can be used to achieve the objective of emissions quality improvement. 


\begin{tabular}{|c|c|c|c|c|c|}
\hline \multicolumn{6}{|c|}{ Table 6.16. Refinery costs in PADD II summer 2010 with average specifications } \\
\hline \multirow[t]{2}{*}{ Case } & \multirow[t]{2}{*}{$\begin{array}{l}\text { Objective } \\
\text { function, }{ }^{\mathrm{a}} \\
\$ \mathrm{M} \text { per day }\end{array}$} & \multirow[t]{2}{*}{$\begin{array}{l}\text { Investment, } \\
\text { \$MM }\end{array}$} & \multirow[t]{2}{*}{$\begin{array}{l}\text { RFD } \\
\text { volume, } \\
\text { MBD }\end{array}$} & \multicolumn{2}{|c|}{$\begin{array}{c}\text { Average cost increase, }{ }^{\mathrm{b}} \\
\text { cents per gallon of RFD } \\
\text { increase relative to: }\end{array}$} \\
\hline & & & & $\begin{array}{c}\text { Base Case } \\
1 \\
\end{array}$ & $\begin{array}{l}\text { Base Case } \\
1 \quad 2 \\
\end{array}$ \\
\hline $\begin{array}{l}\text { Base Case } 1 \\
\text {-Low sulfur on-road diesel } \\
\text { •Investment as required }\end{array}$ & 32,614 & 0.0 & & 0.0 & \\
\hline $\begin{array}{l}\text { Base Case } 2 \\
\text {-ULSD } \\
\text {-Sequential investment (step 1) } \\
\text { - Some newer technologies }\end{array}$ & $\begin{array}{c}30,025 \\
\text { or } \\
32,800 \\
\text { (sunk } \\
\text { investment) }\end{array}$ & 3,644 & 860 & 5.2 & 1 \\
\hline $\begin{array}{l}\text { Case } 2.1 \\
\cdot \text {-ULSD } \\
\text { - Reformulated for vehicle } \\
\text { performance } \\
\text { • Sequential investment (step 2) }\end{array}$ & 31,323 & $\begin{array}{c}1,679 \\
(5,323 \\
\text { cumulative } \\
2 \text { step })\end{array}$ & 863 & 8.4 & 1 \\
\hline $\begin{array}{l}\text { Case } 2.2 \\
\cdot \text { ULSD } \\
\cdot \text { Reformulated for emissions } \\
\text { reduction } \\
\cdot \text {-Sequential investment (step 2) }\end{array}$ & 31,465 & $\begin{array}{c}1,338 \\
(4,982 \\
\text { cumulative } \\
2 \text { step })\end{array}$ & 865 & 8.2 & $\begin{array}{l}1 \\
1 \\
1 \\
1\end{array}$ \\
\hline $\begin{array}{l}\text { a Investment decisions are } \\
\text { b Based on premised actual }\end{array}$ & $\begin{array}{l}\text { on a } 15 \mathrm{p} \\
\text { ounted cas }\end{array}$ & $\begin{array}{l}\text { ont discou } \\
\text { ow ROI o }\end{array}$ & $\begin{array}{l}\text { ash } \mathrm{f} \\
\text { ercen }\end{array}$ & OI. & \\
\hline
\end{tabular}




\begin{tabular}{|c|c|c|}
\hline \multicolumn{3}{|c|}{$\begin{array}{l}\text { Table 6.17. Properties of RFD, with average specifications, in PADD II } \\
\text { summer } 2010\end{array}$} \\
\hline Property & $\begin{array}{l}\text { Case } 2.1 \\
\cdot \text { ULSD } \\
\cdot \text { Reformulated for vehicle } \\
\text { performance } \\
\text { •Sequential investment (step 2) } \\
\end{array}$ & $\begin{array}{l}\text { Case } 2.2 \\
\text {-ULSD } \\
\text { - Reformulated for emissions } \\
\text { reduction } \\
\text { • Sequential investment (step 2) } \\
\end{array}$ \\
\hline Volume, MBD & 863 & 865 \\
\hline API Gravity, ${ }^{\circ} \mathrm{API}$ & 39.0 & 39.2 \\
\hline Specific gravity & .8298 & .8292 \\
\hline Sulfur, ppm & 8 & 8 \\
\hline Cetane Index & 53.5 & 53.9 \\
\hline $\begin{array}{l}\text { Cetane Number, } \\
\text { clear }\end{array}$ & 51.5 & 51.9 \\
\hline $\begin{array}{l}\text { Cetane Improver, } \\
\text { vol } \%\end{array}$ & .043 & .050 \\
\hline $\begin{array}{l}\text { Cetane Improver, } \\
\text { number increase }\end{array}$ & 3.6 & 4.0 \\
\hline Cetane Number, total & 55.1 & 55.9 \\
\hline Aromatics, vol $\%$ & 13.6 & 14.2 \\
\hline Viscosity, cSt & 2.76 & 2.76 \\
\hline ASTM IBP,${ }^{\circ} \mathrm{F}^{\mathrm{a}}$ & 376 & 376 \\
\hline $\mathrm{T} 10$ & 435 & 435 \\
\hline $\mathrm{T} 30$ & 463 & 463 \\
\hline $\mathrm{T} 50$ & 492 & 492 \\
\hline $\mathrm{T} 70$ & 540 & 540 \\
\hline $\mathrm{T} 90$ & 591 & 591 \\
\hline $\mathrm{FBP}^{\mathrm{a}}$ & 635 & 635 \\
\hline NOx change, $\%^{\mathrm{b}}$ & -7.4 & -7.4 \\
\hline PM change, $\%^{\mathrm{b}}$ & -19.6 & -19.6 \\
\hline \multicolumn{3}{|c|}{$\begin{array}{l}\text { aBP and FBP are based on survey reports of differences relative to T10 and T90 } \\
\text { (NIPER 1994). } \\
\text { 'Relative to commercial fuel (Table } 4.1 \text { of Part II), with adjustment for future fleet } \\
\text { change (see II-5.1). }\end{array}$} \\
\hline
\end{tabular}




\section{I-6.8 OFF-ROAD SULFUR}

The off-road sulfur quality giveaway (i.e., exceeding the required sulfur quality) shown in Table 6.18 would be unlikely if off-road quality requirements were coordinated with on-road requirements. In Base Case 1, investments are made for production of low sulfur $(500 \mathrm{ppm}$ nominal; $350 \mathrm{ppm}$ actual $)$ off-road diesel, before notice of requirements for ULSD or RFD. This approach was used to remove or diminish the effects of a variable (off-road sulfur content) in our study.

As previously discussed, we have used the Baker \& O'Brien (2001) projection that up to thirty-eight percent of current on-road low sulfur diesel producers will engage in revamps of distillate hydrotreating units for production of ULSD. Of the remaining hydrotreating capacity for on-road low sulfur diesel, some capacity could be used to produce feedstocks for new deep hydrotreating units for RFD, some capacity could be used to support off-road diesel production, or there could be a reduction in capacity utilization. We have assumed that the remaining hydrotreating capacity continues to operate at Base Case utilization levels. There would be no giveaway of sulfur quality if the utilization of low sulfur hydrotreating capacity were allowed to fall. Other product specifications (e.g., the allowable percentage of light cycle oil) are related to sulfur quality giveaway. For example, if the light cycle oil constraint is removed, there is no sulfur giveaway in any of the products. 


\begin{tabular}{|c|c|c|c|c|}
\hline & Railroad & $\begin{array}{l}\text { Other } \\
\text { Off- } \\
\text { road }\end{array}$ & $\begin{array}{c}\text { Heating } \\
\text { Oil }\end{array}$ & Pool \\
\hline Summer 1996 survey (API/NPRA 1997) & 4,250 & 3,330 & 1,270 & 3,201 \\
\hline RFD study maximum allowable & 350 & 350 & 350 & 350 \\
\hline $\begin{array}{l}\text { Base Case } 1 \\
\cdot \text { LSD } \\
\cdot \text { Investment as required } \\
\end{array}$ & 350 & 350 & 350 & 350 \\
\hline $\begin{array}{l}\text { Case } 1.1 \\
\cdot \text { ULSD } \\
\cdot \text { Reformulated for vehicle performance } \\
\text {-Parallel investment } \\
\end{array}$ & 260 & 330 & 350 & 327 \\
\hline $\begin{array}{l}\text { Case } 1.2 \\
\cdot \text { ULSD } \\
\cdot \text { Reformulated for emissions reduction } \\
\cdot \text { Parallel investment } \\
\end{array}$ & 220 & 350 & 260 & 333 \\
\hline $\begin{array}{l}\text { Base Case } 2 \\
\text {-ULSD } \\
\text {-Sequential investment (step 1) } \\
\text { •Some newer technologies }\end{array}$ & 330 & 280 & 260 & 281 \\
\hline $\begin{array}{l}\text { Case } 2.1 \\
\cdot \text { ULSD } \\
\cdot \text { Reformulated for vehicle performance } \\
\text { - Sequential investment (step 2) } \\
\end{array}$ & 240 & 310 & 350 & 309 \\
\hline $\begin{array}{l}\text { Case } 2.2 \\
\text {-ULSD } \\
\cdot \text { Reformulated for emissions reduction } \\
\text {-Sequential investment (step } 2 \text { ) } \\
\end{array}$ & 240 & 310 & 350 & 309 \\
\hline
\end{tabular}




\section{I-7. IMPACT OF DIESEL FUEL REFORMULATION ON DEMAND FOR CANADIAN SYNTHETIC CRUDE}

In sensitivity cases, ORNL-RYM ${ }_{2002}$ has been used to examine the impacts of RFD on the PADD II refinery demand for Canadian synthetic crude oil. As the production of synthetic crude oil increases, with economic displacement of conventional crude oils, oilsands operators and refiners will face technical challenges with improved quality requirements for diesel fuel, jet fuel, and heavy gas oil for fluid catalytic cracking feed (Yui 2000; Yui and Chung 2001). The cetane quality, aromatics content, and emissions specifications for RFD in our case studies would increase these challenges. Tables 7.1 through 7.7 show fractions and selected properties for the PADD II crude oil used in the case studies; for a Canadian synthetic crude; and for an ORNL-RYM ${ }_{2002}$ approximation of that Canadian synthetic crude. For the distillate fractions, the synthetic crude and its approximation have substantially lower cetane indices $\left(375-690^{\circ} \mathrm{F}\right.$ fraction), and higher aromatics contents $\left(375-650^{\circ} \mathrm{F}\right.$ fraction), compared to the PADD II crude oil. However, with much lower sulfur levels, the value of synthetic crude should increase in the production of low sulfur products.

The sensitivity cases illustrate impacts of changes in diesel product quality requirements, given the premised synthetic crude quality and the PADD II refinery configurations. These cases do not address the issues of (1) modifying the quality of synthetic crude to meet the requirements of crude purchasers; (2) modifying refinery hardware to accommodate higher levels of synthetic crude use; or (3) impacts on product properties (e.g., jet fuel and diesel lubricity) other than those specified in the case studies.

Demand curves are estimated with the ORNL-RYM $\mathrm{M}_{2002}$ parametric feature, which is not appropriate for linking the two steps of the delayed notice, sequential investment pathway. Therefore, demand curves are not presented for sequential Cases 2.1 and 2.2. For the early notice cases, Fig. 7.1 through Fig. 7.3 show the PADD II refinery demand for Canadian synthetic crude for various differentials in its price relative to the price of West Texas Intermediate (WTI) crude.

Fig. 7.1 shows the PADD II refinery demand for Canadian synthetic crude, given production of low sulfur diesel (Base Case 1) and ULSD (Base Case 2). At the actual price differential of $-\$ 0.27$ per barrel in years 2000 and 2001 (Syncrude 2002), the Fig. 7.1 demand for synthetic crude for production of low sulfur diesel (Base Case 1) is $122 \mathrm{MBD}$, or about 2.8 percent of the total PADD II crude run. This synthetic crude percentage is nearly the same as the current actual 2.6 percent (NRC 2002b). With production of low sulfur diesel, the demand for synthetic crude is 0.3 percent of the total PADD II crude run at a price premium to WTI of $\$ 2.00$ per barrel, and 8.5 percent of the total PADD II crude run at a price discount $\$ 2.00$ per barrel. There is a sizeable increase in synthetic crude demand when the maximum sulfur specification for on-road diesel falls from $500 \mathrm{ppm}$ (Base Case 1) to $15 \mathrm{ppm}$ (Base Case 2). This demand increase is expected, given the low sulfur levels of synthetic crude.

Fig. 7.2 shows that diesel fuel reformulation, with challenging specifications for vehicle or emissions reduction, substantially reduces the demand for synthetic crude at recent prices (i.e., in the price differential neighborhood of $-\$ 0.27$ per barrel in years 2000 and 2001), compared with demand under ULSD production. The demand effect increases as price differentials drop below $\$ 0.50$ per barrel. The consolidated Fig. 7.3 shows that diesel fuel reformulation can reverse the synthetic crude demand increase that resulted from the transition from low sulfur diesel to ULSD.

The relationship between desulfurization capacity and synthetic crude demand is illustrated in Fig. 7.4. As expected prices for synthetic crude fall, the expected demand for that crude increases, and investment in firstand second stage deep desulfurization capacity declines. Due to the higher aromatics content of synthetic crude, investment in second stage deep desulfurization/dearomatization capacity increases as the expected demand for synthetic crude increases. 


\begin{tabular}{|c|c|c|c|}
\hline \multicolumn{4}{|c|}{ Table 7.1. Comparison of crude oils in RFD study } \\
\hline \multirow[t]{2}{*}{ Fraction } & \multicolumn{3}{|c|}{ Volume percents } \\
\hline & $\begin{array}{c}\text { PADD II Crude } \\
34.0^{\circ} \text { API } \\
1.27 \% \text { sulfur }\end{array}$ & $\begin{array}{c}\text { Synthetic crude } \\
\text { (NRC 2002a) } \\
31.4^{\circ} \text { API } \\
0.15 \% \text { sulfur }\end{array}$ & $\begin{array}{l}\text { ORNL-RYM } \\
\text { approximation of } \\
\text { synthetic crude } \\
37.3^{\circ} \text { API } \\
0.12 \% \text { sulfur }\end{array}$ \\
\hline $\mathrm{C} 1 / \mathrm{C} 3$ & 0.37 & 0.06 & 0.06 \\
\hline $\mathrm{IC} 4$ & 0.31 & 0.31 & 0.31 \\
\hline $\mathrm{NC4}$ & 1.11 & 1.89 & 1.89 \\
\hline $68-175^{\circ} \mathrm{F}$ & 5.63 & 6.94 & 6.94 \\
\hline $175-250^{\circ} \mathrm{F}$ & 6.73 & 4.69 & \multirow{3}{*}{12.91} \\
\hline $250-325^{\circ} \mathrm{F}$ & 7.13 & 4.82 & \\
\hline $325-375^{\circ} \mathrm{F}$ & 5.05 & 3.40 & \\
\hline $375-500^{\circ} \mathrm{F}$ & 10.55 & 13.88 & \multirow{3}{*}{38.99} \\
\hline $500-550^{\circ} \mathrm{F}$ & 4.21 & 7.93 & \\
\hline $550-650^{\circ} \mathrm{F}$ & 10.85 & 17.18 & \\
\hline $650-690^{\circ} \mathrm{F}$ & 4.64 & 7.17 & 7.17 \\
\hline $690-800^{\circ} \mathrm{F}$ & 11.25 & 16.21 & 16.21 \\
\hline $800-1050^{\circ} \mathrm{F}+$ & 32.16 & 15.51 & 15.51 \\
\hline
\end{tabular}

Table 7.2. Comparison of fraction properties in crude oils of RFD study Fraction: $68-175^{\circ} \mathrm{F}$

\begin{tabular}{||l|c|c|c||}
\hline Fraction & PADD II Crude & $\begin{array}{c}\text { Synthetic crude } \\
\text { (NRC 2002a) }\end{array}$ & $\begin{array}{c}\text { ORNL-RYM } \\
\text { Oppon } \\
\text { aproximation of } \\
\text { synthetic crude }\end{array}$ \\
\hline \hline Gravity, ${ }^{\circ}$ API & 71.5 & 83.1 & 85.0 \\
\hline Aromatics, vol percent & 2.8 & 2.3 & 3.2 \\
\hline Sulfur, wt percent & 0.015 & 0.0032 & 0.0045 \\
\hline
\end{tabular}




\begin{tabular}{|c|c|c|c|}
\hline \multicolumn{4}{|c|}{$\begin{array}{l}\text { Table 7.3. Comparison of aggregated fraction properties in crude oils of RFD study } \\
\text { Fraction: } 175-375^{\circ} \mathrm{F}\end{array}$} \\
\hline Fraction & PADD II Crude & $\begin{array}{l}\text { Synthetic crude } \\
\text { (NRC 2002a) }\end{array}$ & $\begin{array}{l}\text { ORNL-RYM }_{2002} \\
\text { approximation of } \\
\text { synthetic crude } \\
\end{array}$ \\
\hline Gravity, ${ }^{\circ} \mathrm{API}$ & 54.1 & 52.0 & 56.9 \\
\hline Aromatics, vol percent & 14.0 & 17.0 & 17.0 \\
\hline Sulfur, wt percent & 0.048 & 0.0037 & .00037 \\
\hline
\end{tabular}

\begin{tabular}{|c|c|c|c|}
\hline \multicolumn{4}{|c|}{$\begin{array}{l}\text { Table 7.4. Comparison of aggregated fraction properties in crude oils of RFD study } \\
\text { Fraction: } 375-650^{\circ} \mathrm{F}\end{array}$} \\
\hline Fraction & PADD II Crude & $\begin{array}{l}\text { Synthetic crude } \\
\text { (NRC 2002a) }\end{array}$ & $\begin{array}{l}\text { ORNL-RYM } \\
\text { approximation of } \\
\text { synthetic crude }\end{array}$ \\
\hline Gravity, ${ }^{\circ} \mathrm{API}$ & 36.8 & 29.5 & 31.8 \\
\hline Cetane index & 50.4 & 38.3 & 42.5 \\
\hline Aromatics, vol percent & 26.6 & 34.6 & 34.1 \\
\hline Sulfur, wt percent & 0.586 & 0.0391 & 0.0391 \\
\hline
\end{tabular}

\begin{tabular}{|c|c|c|c|}
\hline \multicolumn{4}{|c|}{$\begin{array}{l}\text { Table 7.5. Comparison of fraction properties in crude oils of RFD study } \\
\text { Fraction: } 650-690^{\circ} \mathrm{F}\end{array}$} \\
\hline Fraction & PADD II Crude & $\begin{array}{l}\text { Synthetic crude } \\
\text { (NRC 2002a) }\end{array}$ & $\begin{array}{l}\text { ORNL-RYM } \\
\text { approximation of } \\
\text { synthetic crude }\end{array}$ \\
\hline Gravity, ${ }^{\circ} \mathrm{API}$ & 29.9 & 21.0 & 23.0 \\
\hline Cetane index & 58.0 & 41.7 & 44.4 \\
\hline Sulfur, wt percent & 1.221 & 0.204 & 0.203 \\
\hline
\end{tabular}




\begin{tabular}{|c|c|c|c|}
\hline \multicolumn{4}{|c|}{$\begin{array}{l}\text { Table 7.6. Comparison of fraction properties in crude oils of RFD study } \\
\text { Fraction: } 690-800^{\circ} \mathrm{F}\end{array}$} \\
\hline Fraction & PADD II Crude & $\begin{array}{l}\text { Synthetic crude } \\
\text { (NRC 2002a) }\end{array}$ & $\begin{array}{l}\text { ORNL-RYM }_{2002} \\
\text { approximation of } \\
\text { synthetic crude } \\
\end{array}$ \\
\hline Gravity, ${ }^{\circ} \mathrm{API}$ & 26.2 & 19.9 & $26.1^{\mathrm{a}}$ \\
\hline Sulfur, wt percent & $0.949^{\mathrm{a}}$ & 0.298 & 0.298 \\
\hline
\end{tabular}

\begin{tabular}{|c|c|c|c|}
\hline \multicolumn{4}{|c|}{$\begin{array}{l}\text { Table 7.7. Comparison of fraction properties in crude oils of RFD study } \\
\text { Fraction: } 800-1050^{\circ} \mathrm{F}+\end{array}$} \\
\hline Fraction & PADD II Crude & $\begin{array}{l}\text { Synthetic crude } \\
\text { (NRC 2002a) }\end{array}$ & $\begin{array}{l}\text { ORNL-RYM }_{2002} \\
\text { approximation of } \\
\text { synthetic crude }\end{array}$ \\
\hline Gravity, ${ }^{\circ} \mathrm{API}$ & 15.5 & 17.9 & 17.1 \\
\hline Sulfur, wt percent & 2.03 & 0.363 & 0.320 \\
\hline
\end{tabular}


Fig. 7.1. ULS Diesel Increases Demand for Synthetic Crude

(PADD II Summer 2010)

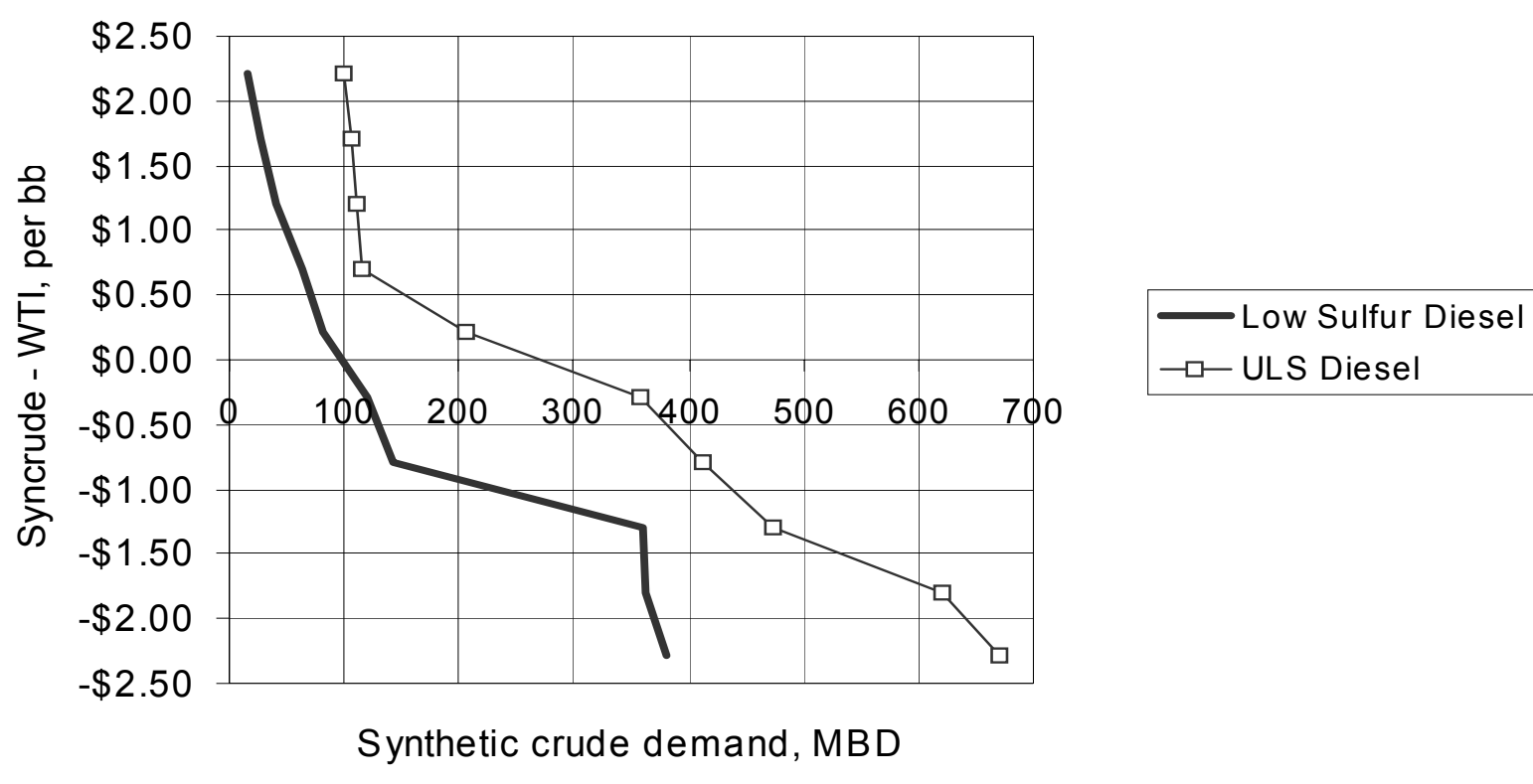

Fig. 7.2. RFD Reduces Demand for Synthetic Crude at Recent

Prices: Parallel Investment

(PADD II Summer 2010)
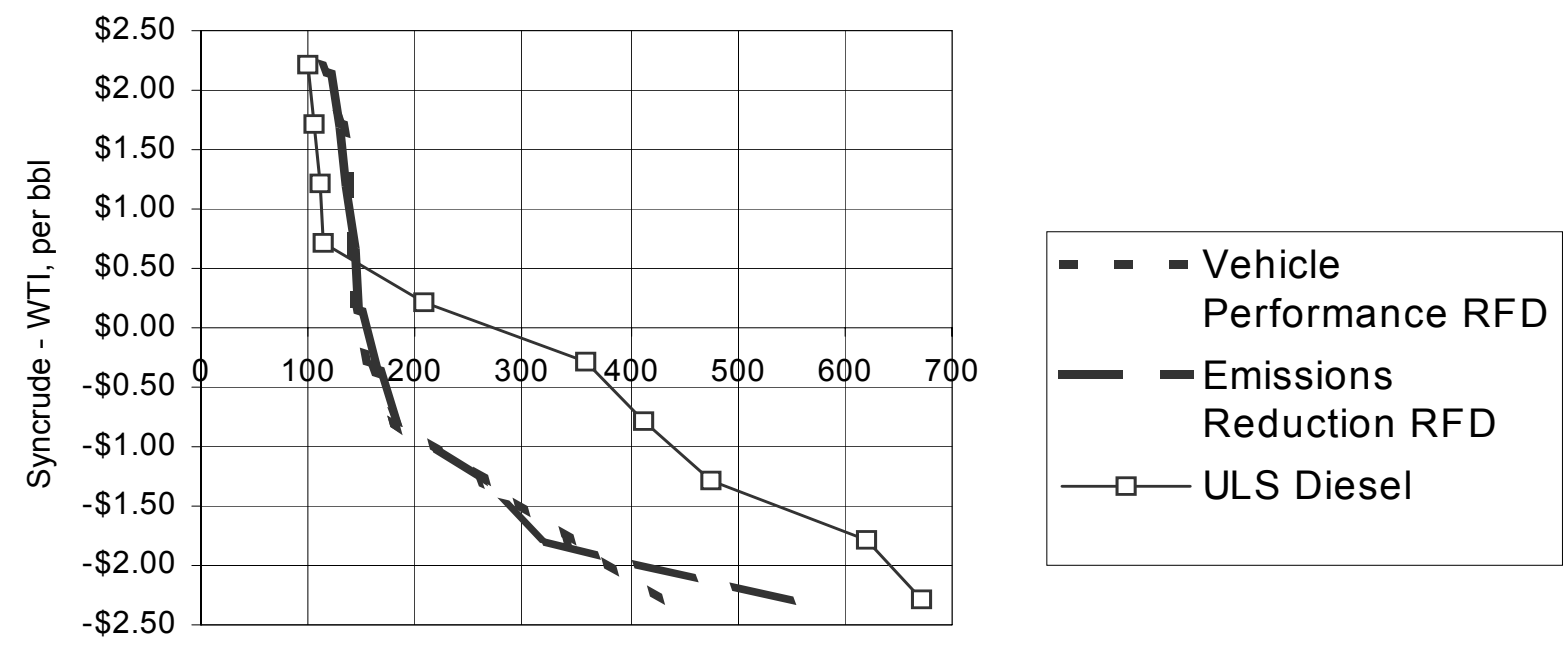

Synthetic crude demand, MBD 

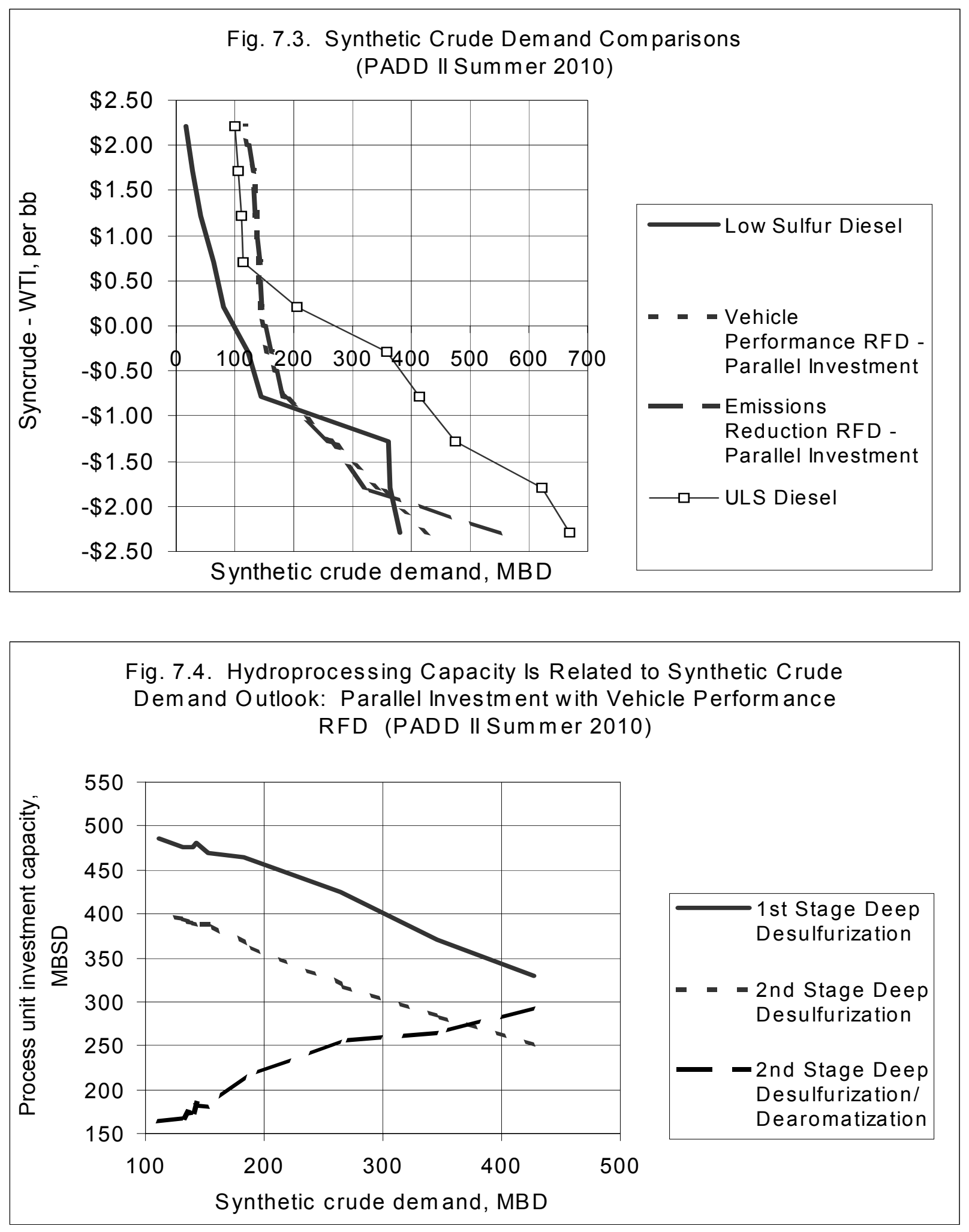


\section{I-8. CONCLUSIONS}

ORNL-RYM ${ }_{2002}$ has been used to estimate the refining cost and investment impacts of different diesel fuel reformulation pathways, to determine if there are refinery economic benefits for producing an emissions reduction RFD (with eigenfuel-based flexibility for individual property values) compared to a vehicle performance RFD (with recipe values for individual properties). These RFDs are premised to have equal emissions performance.

Table 8.1 shows that refining costs are lower with early notice of product quality requirements for RFD. While advanced desulfurization technologies (with low hydrogen consumption and little effect on cetane quality and aromatics content) reduce the cost of ULSD, these technologies contribute to the increased costs of a delayed-notice sequential investment pathway compared to an early-notice parallel investment pathway to RFD.

Given the objective of RFD emissions quality improvement, there is little refining economic benefit from using the flexible emissions reduction specifications rather than the vehicle performance recipe specifications in these cases, which have very challenging specifications. As specifications become tighter, processing becomes more difficult, and blendstock choices become more limited, refinery benefits vanish for emissions reduction relative to vehicle performance specifications.

Conversely, the emissions reduction specifications show increasing refinery benefits over vehicle performance recipe specifications as those recipe specifications are relaxed, and alternative processing routes and blendstocks become available. For example, using average specifications rather than cap specifications in our study reduces investment and RFD production costs for emissions reduction compared to vehicle performance. With the increased blending flexibility of emissions reduction specifications for RFD, there are changes in API gravity, cetane number and/or aromatics content, while the same emissions quality is achieved for both emissions reduction and vehicle performance RFDs.

In sensitivity cases, ORNL-RYM $\mathrm{O}_{2002}$ indicates that there is a sizeable increase in synthetic crude demand as ULSD displaces low sulfur diesel fuel, but this demand increase would be reversed by a requirement for RFD.

In summary:

- Refining costs are lower with early notice of product quality requirements for RFD. With early notice of RFD requirements, refinery capital investment has not yet been made to satisfy the ULSD requirement of year 2006, and there is greater flexibility for investment planning. With delayed notice of requirements, investment flexibility is less because capital investment has been sunk to satisfy the ULSD sulfur requirement.

- While advanced desulfurization technologies reduce the cost of ULSD, these technologies can contribute to the increased costs of a delayed notice compared to an early notice investment pathway to RFD. With relatively small impact on cetane and aromatics quality, these technologies are on a suboptimal pathway for RFD production.

- There is little refining economic benefit from using flexible emissions reduction specifications rather than the vehicle performance recipe specifications, when recipe specifications are challenging. This is because processing options and blendstocks are very limited for achieving challenging recipe specifications. 
- Emissions reduction specifications show increasing refinery benefits over vehicle performance recipe specifications, as those recipe specifications are relaxed, and alternative processing routes and blendstocks become available.

- There is a sizeable increase in synthetic crude oil demand as ULSD displaces low sulfur diesel fuel, but this demand increase would be reversed by a requirement for diesel fuel reformulation. 


\begin{tabular}{|c|c|c|c|}
\hline \multicolumn{4}{|c|}{ Table 8.1. Diesel fuel reformulation study findings } \\
\hline Case & $\begin{array}{c}\text { On-road diesel } \\
\text { upgrading pathway } \\
\end{array}$ & $\begin{array}{c}\text { Cost } \\
\text { increase }^{\mathrm{a}} \\
\end{array}$ & Comment \\
\hline Base Case 1 & $\begin{array}{l}\text {-Low sulfur } \\
\text { •Investment as required }\end{array}$ & 0.0 & \\
\hline Case 1.1 & $\begin{array}{l}\text {-Ultra low sulfur } \\
\text { - Reformulated for } \\
\text { vehicle performance } \\
\text {-Early notice of } \\
\text { requirements } \\
\text {-Parallel investment }\end{array}$ & 9.0 & $\begin{array}{l}\text { Due to high cetane specification, distillate upgrading is } \\
\text { via 2-stage deep hydroprocessing, not advanced } \\
\text { desulfurization technologies that selectively remove } \\
\text { sulfur without cetane improvement. }\end{array}$ \\
\hline Case 1.2 & $\begin{array}{l}\text {-Ultra low sulfur } \\
\text { - Reformulated for } \\
\text { emissions reduction } \\
\text {-Early notice of } \\
\text { requirements } \\
\text {-Parallel investment }\end{array}$ & 8.9 & $\begin{array}{l}\text { Emissions targets are based on Case } 1.1 \text { (relative to } \\
\text { reference commercial fuel, NOx reduction } \geq 8.3 \text { percent } \\
\text { and PM reduction } \geq 19.8 \text { percent). With little } \\
\text { blendstock flexibility (virtually same stocks selected as } \\
\text { in Case 1.1), emissions reduction specifications have } \\
\text { little refining benefit, given equal emissions for the two } \\
\text { RFDs. }\end{array}$ \\
\hline Base Case 2 & $\begin{array}{l}\text {-Ultra low sulfur } \\
\text {-Sequential investment } \\
\text { (step 1) } \\
\text {-Some newer } \\
\text { technologies }\end{array}$ & 5.2 & $\begin{array}{l}\text { Investment in advanced desulfurization technology } \\
\text { results in lower costs. Sensitivity runs show cost } \\
\text { increase of } 7.9 \mathrm{cpg} \text { via } 2 \text {-stage deep hydroprocessing, } \\
\text { with no investment allowed in advanced desulfurization; } \\
\text { and cost increase of } 3.7 \mathrm{cpg} \text { with no constraints on } \\
\text { investment in advanced desulfurization. }\end{array}$ \\
\hline Case 2.1 & $\begin{array}{l}\text {-Ultra low sulfur } \\
\text { - Reformulated for } \\
\text { vehicle performance } \\
\text {-Delayed notice of } \\
\text { requirements } \\
\text {-Sequential investment } \\
\text { (step 2) }\end{array}$ & 9.7 & $\begin{array}{l}\text { In second step to high cetane/low aromatics, there is } \\
\text { drop in utilization of advanced desulfurization, and } \\
\text { refineries with this capacity must purchase high } \\
\text { cetane/low aromatics stocks from those refineries with } \\
\text { 2-stage deep hydroprocessing and/or invest in distillate } \\
\text { dearomatization capacity. Capital investment increases, } \\
\text { and cost increases } 0.7 \mathrm{cpg} \text { compared to early notice } \\
\text { pathway of Case } 1.1 .\end{array}$ \\
\hline Case 2.2 & $\begin{array}{l}\text {-Ultra low sulfur } \\
\text { - Reformulated for } \\
\text { emissions reduction } \\
\text {-Delayed notice of } \\
\text { requirements } \\
\text {-Sequential investment } \\
\text { (step 2) }\end{array}$ & 9.7 & $\begin{array}{l}\text { Emissions targets are based on Case } 2.1 \text { (relative to } \\
\text { reference commercial fuel, NOx reduction } \geq 8.2 \text { percent } \\
\text { and PM reduction } \geq 19.8 \text { percent). There are no refinery } \\
\text { benefits from using emissions versus vehicle } \\
\text { performance specifications in these cases with very } \\
\text { challenging specifications. As specifications become } \\
\text { tighter, refinery benefits vanish for emissions } \\
\text { reduction versus vehicle performance specifications. } \\
\text { Conversely, emissions reduction specification benefits } \\
\text { for refining increase as vehicle performance } \\
\text { specifications are relaxed. }\end{array}$ \\
\hline $\begin{array}{l}\text { Synthetic } \\
\text { Crude } \\
\text { Sensitivities }\end{array}$ & \multicolumn{3}{|c|}{$\begin{array}{l}\text { There is a sizeable increase in synthetic crude demand when the maximum sulfur specification for } \\
\text { on-road diesel falls from } 500 \mathrm{ppm} \text { to } 15 \mathrm{ppm} \text {, but this demand increase would be reversed by } \\
\text { diesel fuel reformulation. }\end{array}$} \\
\hline
\end{tabular}





\section{REFERENCES}

American Petroleum Institute and National Petroleum Refiners Association (API/NPRA). 1997. 1996 American Petroleum Institute/National Petroleum Refiners Association Survey of Refining Operations and Product Quality, API Publishing Services, 1220 L. Street, N.W., Washington DC, July.

Clark, Dale (NRC). 2002a. Data on synthetic crudes and Canadian heavy crudes, Natural Resources Canada, Ottawa, Ontario.

Crawford, R.W. 2002. "Development of NOx Emissions Model for Heavy-Duty Diesel Engines," RWCrawford Energy Systems, under contract with Oak Ridge National Laboratory, April 16.

Dickson, Cheryl L. and Gene P. Sturm, Jr. (NIPER). 1994. Diesel Fuels, 1994, NIPER-187 PPS 94/5, National Institute for Petroleum and Energy Research, Bartlesvillle, OK, December.

Downstream Alternatives, Inc (DAI). 2002. Infrastructure Requirements for an Expanded Fuel Ethanol Industry, Oak Ridge National Laboratory Ethanol Project, Subcontract No. 4500010570, South Bend, IN 46680-2587, January 15, http://www.afdc.doe.gov/pdfs/6235.pdf

Dunbar, Daniel N. and Martin R. Tallett. 2002a. "Progress Report Number 1 - Technology and Features Enhancements to ORNL RYM,” EnSys Energy \& Systems, Inc., Flemington, NJ.

Dunbar, Daniel N. and Martin R. Tallett. 2002b. "Progress Report Number 2 - Technology and Features Enhancements to ORNL RYM,” EnSys Energy \& Systems, Inc., Flemington, NJ, April 7.

European Automobile Manufacturers Association, Alliance of Automobile Manufacturers, Engine Manufacturers Association, Japan Automobile Manufacturers Association (ACEA et al). 2000. "World-wide Fuel Charter," April, http://www.engine-manufacturers.org/about/Final\%20WWFC\%204\%2000.pdf

Hadder, G.R. 1997. Re-estimation of the Refining Cost of Reformulated Gasoline NOx Control, draft report, Oak Ridge National Laboratory, Oak Ridge, TN, February 26.

Korotney, D. 1993. "Revised Complex Model for Conventional and Reformulated Gasoline," EPA Air Docket A-92-12, Environmental Protection Agency, Fuels and Standards Branch, Ann Arbor MI.

MathPro Inc. 2002. Prospects for Adequate Supply of Ultra Low Sulfur Diesel Fuel in the Transition Period (2006-2007), Prepared for The Alliance of Automobile Manufacturers and The Engine Manufacturers Association, West Bethesda, MD, February 26.

McAdams, H.T., R.W. Crawford, and G.R. Hadder. 2000a. "A Vector Approach to Regression Analysis and Its Application to Heavy-Duty Diesel Emissions," SAE 2000-01-1961, Society of Automotive Engineers International Spring Fuels \& Lubricants Meeting \& Exposition, Paris, France, June 19-22.

McAdams, H.T., R.W. Crawford, and G.R. Hadder. 2000b. A Vector Approach to Regression Analysis and Its Application to Heavy-Duty Diesel Emissions, ORNL/TM-2000/5, Oak Ridge National Laboratory, Oak Ridge, TN 37831, November.

McAdams, H.T., R.W. Crawford, and G.R. Hadder. 2002. PCR + in Diesel Fuels and Emissions Research, ORNL/TM-2002-16, Oak Ridge National Laboratory, Oak Ridge, TN 37831, March. 
National Petroleum Council (NPC). 1993. U.S. Petroleum Refining, Washington, DC, August.

National Petroleum Council (NPC). 2000. U.S. Petroleum Refining, Assuring the Adequacy and Affordability of Cleaner Fuels, Washington, DC, June.

National Petroleum Refiners Association (NPRA). 1991. NPRA Survey of U.S. Gasoline Quality and U.S. Refining Industry Capacity to Produce Reformulated Gasolines, Part A, Suite 1000, 1899 L Street, NW, Washington, DC, January.

Natural Resources Canada (NRC). 2002b. “Canadian Oil Sands,” TRB Summer Meeting, Port Huron, MI, July.

Ory, Raymond E., Jr (Baker \& O'Brien). 2001. "Potential Impacts of Sulfur Regulation on Cost and Supply of Diesel Fuels in the United States," National Petrochemical \& Refiners Association, AM-01-32, Washington, DC.

Shapiro, Ellen. 2002. "Comment on Draft DOE Study Protocol," Alliance of Automobile Manufacturers, e-mail to Gerald Hadder, May 29.

Shiflett, Woody. 2001. "The Drive to Lower and Lower Sulfur: Criterion's New Catalysts Help Refiners Tackle Sulfur," National Petrochemical \& Refiners Association, AM-01-29, Washington, DC.

Sinor, J.E. 2000. Review of Market for Octane Enhancers, J.E. Sinor Consultants, Inc., Niwot, CO, May, http://www.afdc.doe.gov/pdfs/4747.pdf

Stell, Jeannie. 2001a. "Worldwide Construction," Oil \& Gas Journal, October 29, http://downloads.pennnet.com/pnet/surveys/ogj/ogj_99447295.pdf

Stell, Jeannie. 2001b. "2001 Worldwide Refining Survey," Oil \& Gas Journal, December 24, http://downloads.pennnet.com/pnet/surveys/ogj/ogj_9952jp082-124.pdf

Swain, Edward J. 1999. "U.S. refinery-sulfur production peaked in 1996," Oil \& Gas Journal, March 8.

Syncrude Canada LTD (Syncrude). 2002. Syncrude Canada LTD 2001 Annual Report. Fort McMurray, Alberta, http://www.syncrude.com/investors/ar01/pdf/FinRev.pdf

Tallett, M.R., and D.N. Dunbar. 1988. Enhancement of EIA Refinery Yield Model: Extension and Demonstration on Gasoline and Diesel Quality Issues, Oak Ridge National Laboratory Contract No. DE-AC05-840R21400, EnSys Energy \& Systems, Inc., Flemington, NJ, August.

Tallett, M.R. and D.N. Dunbar. 2001. "Proposal for Technology and Features Enhancements to the ORNL RYM Model,” EnSys Energy \& Systems, Inc., Flemington, NJ, December 4.

Tallett, M.R., D.N. Dunbar, and J. Leather. 1992. "Summary Documentation of Oak Ridge Refinery Yield Model Reformulated Gasoline Update,” EnSys Energy \& Systems, Inc., Flemington, NJ, March 16.

U.S. Department of Energy (DOE). 1984a. Refinery Evaluation Modeling System (REMS) Documentation, DOE/EIA-0460, Energy Information Administration, Washington, DC, October. 
U.S. Department of Energy (DOE). 1984b. Refinery Evaluation Modeling System (REMS) Documentation, DOE/EIA-0461, Energy Information Administration, Washington, DC, October.

U.S. Department of Energy (DOE), Energy Information Administration. 2001a. Annual Energy Outlook 2002 with Projections to 2020, DOE/EIA-0383(2002), Washington, DC, December 21, http://www.eia.doe.gov/oiaf/aeo/pdf/appa.pdf

U.S. Department of Energy (DOE), Energy Information Administration. 1998. Challenges of Electric Power Industry Restructuring for Fuel Suppliers, DOE/EIA-0623, Washington, DC, September, http://www.eia.doe.gov/pub/electricity/chg_str_fuel.pdf

U.S. Department of Energy (DOE), Energy Information Administration. 2002a. Petroleum Marketing Annual, DOE/EIA-0380(2001), http://www.eia.doe.gov/oil_gas/petroleum/data_publications/petroleum_marketing_annual/pma.html

U.S. Department of Energy (DOE), Energy Information Administration. 2001b. Petroleum Supply Annual 2000, DOE/EIA-0340(00)/1 and 2, Washington, DC, June, http://www.eia.doe.gov/oil_gas/petroleum/data_publications/petroleum_supply_annual/psa_volume1/psa volume1.html and

http://www.eia.doe.gov/oil_gas/petroleum/data_publications/petroleum_supply_annual/psa_volume2/psa _volume2.html

U.S. Department of Energy (DOE), Energy Information Administration. 2002b. Refinery Capacity Data as of January 1, 2001, DBF file containing petroleum refinery capacity data by refinery. Energy Information Administration (EIA), Form EIA-820, "Biennial Refinery Report," http://www.eia.doe.gov/oil_gas/petroleum/data_publications/refinery_capacity_data/refcapacity.html.

U.S. Environmental Protection Agency (EPA). 2001. Control of Emissions of Hazardous Air Pollutants from Mobile Sources, 40 CFR Parts 80, and 86.

U.S. Environmental Protection Agency (EPA). 2002. "Toxics baselines," e-mail memorandum and spreadsheet attachment from John Weihrauch to Gerald Hadder, June 27.

Weihrauch, John. 2001. "Revised PADD Data," tables provided to G.R. Hadder.

Yui, Sok. 2000. "Athabasca oilsands produce quality diesel and jet fuels," Oil \& Gas Journal, November 20.

Yui, Sok and Keng Chung. 2001. "Processing oilsands is Syncrudes's R\&D focus," Oil \& Gas Journal, April 23. 



\section{APPENDIX I-A}

ENSYS ENERGY \& SYSTEMS, INC., PROGRESS REPORT NUMBER 1 TECHNOLOGY AND FEATURES ENHANCEMENTS TO THE ORNL RYM MODEL 



\section{EnSys ORNL Contract: 4000013512 - ORNL Refinery Yield Model Update}

\section{Progress Report Number 1- Technology and Features Enhancements to the ORNL RYM Model}

\section{Hydrocracking/FCC Feed Pretreating}

The RYM structure as now used provides for:

$\begin{array}{lc}\text { Unit FDS } & \text { FCC Feed pretreating } \\ \text { Unit HCL } & \text { Mild to moderate hydrocracking } \\ \text { HCR } & \text { Severe hydrocracking } \\ \text { HCV } & \text { Residuum hydrocracking }\end{array}$

The above correspond to the Oil and Gas Journal annual refining survey categories.

The Exxon Mobil MAK process table MAK is retained as a separate unit. In using the model, the above categories suffice since they cover the range of hydrocracking severity reflected in Table MAK. However since the MAK table exists, the table values are being reviewed and updated based on the latest NPRA papers. Its structure will not be expanded.

Although the current structure holds, some table vectors are being moved from Table FDS to HCL. A total of 20 additional process unit vectors have been defined to fit in the above structure based on vendor data appearing in Hydrocarbon Processing for UOP and Chevron, a Texaco NPRA Paper, a NPRA Paper describing the UOP process and data from Handwerk, Maples and Meyers. A (spreadsheet) document will be supplied detailing the precise sources.

Hydrogen consumptions range from 650 to $2500 \mathrm{SCF} / \mathrm{bbl}$ (before allowing for hydrogen losses) and product middle distillate sulfur contents range from 10 to $400 \mathrm{ppm}$. There are vectors which correspond to LCO and light FCC and coker gas oils hydrocracking. The LCO data indicate hydrogen consumption of the order of $2000 \mathrm{scf} / \mathrm{bbl}$ or greater and a conversion range from 30 to 60 percent. Undercutting of the LCO will not be further considered since both FCC distillate and light gas oil feed are represented and undercutting appears to fall within the realm of refinery operations sub-optimization.

The existing approach of hydrocracker vectors corresponding to a gasoline versus a jet/distillate operation is being reviewed and will be maintained.

\section{Hydrogen Plant Revamp}

Three NPRA papers were reviewed - AM-01-19, Air Products/Tecnip, AM-01-27, Haldor Topsoe and AM01-27, Foster Wheeler, AM-01-36. We estimate that a 30\% increase in capacity can be achieved for $25 \%$ of the ISBL and $10 \%$ of the OSBL investment costs, with a marginal increase in energy efficiency of $8 \%$.

There are other options aside from de-bottlenecking: 
Retirement and replacement - According to Air Products, during the period 1991 through 2000, refiners added approximately 300 MMSCFD of new capacity and retired 130 MMSCFD. Although Foster Wheeler states that the tighter new plant designs offer less revamp potential than the older plants, on the other hand they note many of the older plants have been revamped at least once. From the above figure for outright hydrogen plant retirement, the revamp potential is definitely limited.

Across the fence hydrogen purchase. During the same 1991-2000 period, industrial gas companies added 850 MMSCFD of capacity for sale to refiners. This option is becoming attractive to refiners because they tend to avoid the (now near catastrophic) consequences of a sudden hydrogen plant shutdown, they avoid expending investment capital and they have greater refinery operations flexibility.

Hydrogen Recovery and Purification - this is discussed below.

The modeling challenges for representing the important cost of hydrogen appear to lie less in estimating the cost of the various supply options than in estimating their likely adoption. We are considering the best option could be to represent plant "revamp" in fact as a debottleneck with controlled upper limits.

\section{Hydrogen Purification and Recovery}

Two units are being considered - the existing HPM unit for membrane purification and a new RYM Table PSA to represent pressure swing absorption. The latter is gaining ground in spite of a higher investment cost since higher purity hydrogen is produced at a higher pressure. Investment cost data has been assembled from Hydrocarbon Processing, UOP NPRA Paper AM-99-08 and European Union data available from the Internet.

If both units are allowed to ride free, the validity of the relative selection may be in question. The higher purity of the PSA hydrogen facilitates middle distillate desulfurization to lower sulfur levels (one reason for its selection), but we do not represent different hydrogen purity levels in RYM. A disadvantage of the membrane process is the need for hydrogen recompression and we do not represent different hydrogen pressure levels in RYM, although we can make an adjustment to the HPM investment cost. Also, total hydrogen purification may exceed reasonable values since site specific limitations are a reality.

We propose to put in Table POL constraints to limit both total hydrogen purification capacity and PSA versus HPM - current OGJ refinery survey data indicate the hydrogen purification capacity accounts for $38 \%$ of hydrogen plant production and the survey shows HPM versus PSA (cryogenic separation is relatively minor).

\section{Hydrogen Consumption Consistency Across Process Units}

Based on published data, the RDS and ARD units show reasonable hydrogen consumption values. However, the residuum hydrocracker is low by an estimated factor of 1.75 based on data from Hydrocarbon Processing an Handwerk. The middle distillate hydrogen consumption data are being reviewed separately as a part of the following task 


\section{$\underline{\text { V. Middle Distillate Deep Desulfurization and Aromatics Saturation }}$}

Building on the recent model development work for API, two NPRA Papers were reviewed in depth: AM-0122 by Mustang Engineers and Constructors, Inc and AM-01-28 by Haldor Topsoe and San Joaquin Refining Company.

These papers address revamp potential and the incremental steps required to achieve less than $10 \mathrm{ppm}$ product sulfur. The Mustang paper gives ULSD revamp cost estimates. The requirements for aromatics saturation are also addressed in these papers.

The two papers essentially agree on the revamp steps required to achieve ULSD:

the addition of a second reactor or a new second stage hydrotreater to lower space velocity by providing increased catalyst volume

more active catalyst directed at desulfurizing sterically hindered sulfur compounds (look at NiMo versus CoMo).

higher hydrogen purity from hydrogen purification

scrubbing of the recycle gas to provide higher hydrogen purity

higher reactor pressure to be considered in addition to the above to increase the hydrogen partial pressure in the reactor

The reactor internals must also be examined since a one percent bypassing of the feed can contribute 50 to $150 \mathrm{ppm}$ sulfur content to the diesel product.

In order to saturate aromatics to the 4 weight percent level, increase feed cetane by 10 numbers and API by 6 , a second high pressure noble catalyst stage is required, along with an intermediate stripper column to remove dissolved $\mathrm{H} 2 \mathrm{~S}$ and $\mathrm{NH} 3$ from the first stage effluent

\section{$\underline{\text { Revamp Potential }}$}

Both NPRA papers state that units built since the early 1990s may be revamped according to the above scheme and that additional revamps of the type above to already revamped units may not be feasible. To revamp an earlier generation hydrocracker (1970's and 1980's) may require up to three times the catalyst volume, as opposed to an increase of 100 percent in the catalyst volume for the post 1990 units.

\section{$\underline{\text { Revamp Cost }}$}

For a 30,000 BPD unit with 1/3 LCO feed, Mustang estimates 8.6 million dollars for the above sequence for a $100 \%$ catalyst volume increase. This is an ISBL figure with an accuracy stated of 20 percent up or down. An offsites cost range of 30-100 percent onsite investment is quoted. The Mustang paper indicates a 0.9 million dollar ISBL increase in going from a 50 to 100 percent catalyst volume increase. Applying economies of scale, this provides a basis for estimating the revamp cost of a pre-1990s hydrotreater requiring three times the catalyst volume.

$\underline{\text { Implementation }}$

Through EnSys' work for the API, we have established limits and cost factors for revamping existing LSD units (Table SYD) and existing conventional DDS units into ULSD units. We are using the above published updates to tune the revamp cost factors we have in the model. 


\section{Next Steps}

With the above steps now undertaken, we are moving into the second stage of the tasks namely to build in the changes and to implement the other RYM feature updates.

Daniel Dunbar

Martin Tallett 


\section{APPENDIX I-B}

ENSYS ENERGY \& SYSTEMS, INC., PROGRESS REPORT NUMBER 2 TECHNOLOGY AND FEATURES ENHANCEMENTS TO THE ORNL RYM MODEL 

EnSys Energy \& Systems, Inc.

\title{
EnSys ORNL Contract: 4000013512 - ORNL Refinery Yield Model Update
}

\author{
Progress Report Number 2 - Technology and Features Enhancements \\ to the ORNL RYM Model
}

Additions and changes to RYM, including Table INVUNT have been completed, i.e. all the modifications reviewed below have been built in to the RYM master Excel workbook. A shakedown test run during Task B is planned to reveal the need for any corrections.

The existing middle distillate streams proved adequate to accomplish the enhancements listed below. This will not be true for the next stage where the RYM enhancements are to be extended to very low sulfur gasoline. The updates and additions include the following:

\section{Hydrocracking/FCC Feed Pretreating}

The RYM structure has been established to correspond to:

Unit FDS FCC Feed pretreating

Unit HCL Mild to moderate hydrocracking

HCR Moderate to Severe hydrocracking

HCU New generation hydrocracking, capable of producing naphtha and distillate streams at the 10 ppm sulfur level.

The above correspond to the Oil and Gas Journal annual refining survey categories, however premise judgement will have to be exercised in specifying the extent of HCU units. While the hydrocracker representation maintains the flexibility to operate in a gasoline versus jet versus distillate mode, all of the RYM process tables have been reworked to reflect the growing importance of this unit in a low sulfur world.

Table HCR Moderate to Severe Hydrocracking

This is based on pre-2000 technology and with product qualities approximating the July 1997 "API Survey of Refining Operations and Product Quality". A new Table HCU represents advanced state of the art hydrocracking designed to attain $10 \mathrm{ppm}$ sulfur level middle distillate and naphtha sulfur levels, accompanied by de-aromatization and cetane boosting. The existing table HCR has been completely revised - data are based primarily on UOP Unicracking 


\section{EnSys Energy \& Systems, Inc.}

published in the November 2000 issue of Hydrocarbon Processing, page 118. Table HCR contains an option to hydrocrack light cycle oil with data based on Mobil NPRA paper AM-9539 with an LCO conversion of $60 \%$.

Table HCU New Generation Hydrocracking Technology

This new table is for advanced hydrocracking technology claimed to increase middle distillate yield by 5 to $15 \%$ with middle distillate $10 \mathrm{ppm}$ sulfur level products, $5-15$ percent aromatics content and at the 60 cetane number level. The process employs an efficient means of recycling unconverted oil to the cracking reactor, an enhanced hot separator and back-staged reactors. The process is claimed to economize on hydrogen consumption. Data are based primarily on a UOP description published in NPRA paper AM-01-30 and IFP process data published in Hydrocarbon processing, November 2000, page 116. These high product qualities are attained with Arab Medium crude oil, with $3.0 \%$ sulfur gas oil

Table HCU also contains an option to hydrocrack light cycle oil to a sub $10 \mathrm{ppm}$ level distillate sulfur level.

An option that can now be considered is to allow revamping of existing HCR units to HCU. We do not currently have an assessment of the extent to which this is feasible.

\section{Table HCL Mild/Low Conversion Hydrocracking}

Data are based on a Hydrocarbon Processing article, November 1999, p 43, Tables 10 and 11 for partial (low) conversion operations. Also on the Texaco T-Star mild hydrocracking process as described in AM-6-60 corresponding to 32 percent gas oil conversion. By comparison, RYM Table FDS to hydrotreat FCC feed is now structured to accomplish desulfurization, with an optional hydrocracking conversion function limited to $15 \%$ gas oil conversion.

Table MAK Exxon Mobil Makfining

The Exxon Mobil MAK process MAK remains in RYM as a hydrocracking alternative and reflects 55 percent gas oil conversion. In using the model, the above categories suffice since they cover the range of hydrocracking severity reflected in Table MAK. Since Table MAK remains, the MAK table values and investment cost have been reviewed against Exxon Mobil/Kellogg Brown \&Root NPRA Papers AM-97-64 and AM-00-18. 


\section{EnSys Energy \& Systems, Inc.}

In addition to the sources cited above, process and investment cost estimates have been reviewed and incorporated from vendor data appearing in Hydrocarbon Processing for UOP and Chevron and from Handwerk, Maples and Meyers. Specific reference detail will be provided in the documentation product to be delivered.

The above process unit tables contain process vectors which are for virgin light and heavy gas oils, coker gas oil and FCC LCO. Undercutting of the LCO will not be further considered since it appears to fall within the realm of refinery operations sub-optimization.

\section{FCC Yield and Product Quality Effects}

The input of hydrotreated and hydrocracked feed to the FCC unit of course lower the sulfur level of the FCC naphtha and distillate products, while increasing FCC gas oil conversion. These effects were reviewed and also updated to reflect the changes made to RYM as described above. The data were based on Hydrocarbon Processing, November 1999, page 43 (UOP), Harts Fuel Technology \& management, November/December 1999, page 59 (Azko Nobel Chemicals) and Criterion Catalyst NPRA paper AM-01-29.

\section{Hydrogen Consumption Consistency Across Process Units}

Based on published data, the hydrogen consumption values have been updated. The hydrogen consumption levels of ARD, RDS and HCV have been raised by factors of 1.8, 1.5 and 1.2 respectively.

A comparative summary of process unit hydrogen consumption levels as they stand now in RYM is given below:

\section{Hydrogen Consumption SCF/bbl}

ARD -Atmospheric Resid Desul

RDS - Residuum Desulfurization

HCV- Residuum Hydrocracking

FDS - FCC Feed Desulfurization

HCL- Mild Hydrocracking

HCR- Moderate to Severe Hydrocracking

HCU- New Generation Technology

MAK- Exxon Mobil Makfining

SYD - Synsat for Diesel

\section{$\underline{\text { Virgin Feed }}$}

900

1200

1450

450

600-900

1200-2000

2500

$1150-1400$

400-500

\section{$\underline{\mathrm{LCO} \text { or } \mathrm{LCGO}}$}

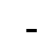

-

550

2100-2500

2800

1700

1370 


\section{EnSys Energy \& Systems, Inc.}

$\begin{array}{lll}\text { HD1 - First Stage Deep Desulfurization } & 110(1) & 300-500 \\ \text { HS2 - Second Stage Deep Desulfurization } & 350 & 300-500 \\ \text { HD2 - Stage 2-Dearomatization } & 700 & 900-1700 \\ & & 280\end{array}$

(1) On review, the hydrogen consumption for HDl virgin feed appears low. Actual HD1 hydrogen consumptions vary depending on the type of feedstock (kero, heavy kero, diesel B) and the feed sulfur level. We will check whether this effectively is a typo or whether the hydrogen consumption range needs to be altered.

\section{Review of Investment Costs}

The capital investment estimates of all RYM process units involved in the production of ultra low sulfur diesel fuel were reviewed, with the results shown below and as in Table INVUNT. In addition to the specific references cited in the sections below, the principal sources were Gary and Handwerk, $4^{\text {th }}$ Edition and Robert E. Maples, $2^{\text {nd }}$ Edition. Detailed source pages will be provided in the documentation to be provided. The deep desulfurization/dearomatization capital costs were thoroughly reviewed in connection with a recent ULSD study for API and an EnSys report in conjunction with Dr. Richard Foley for EIA.

$\begin{array}{lcc} & \begin{array}{c}\text { Size } \\ \text { Bpd }\end{array} & \begin{array}{c}\text { ISBL Investment } \\ \text { MM Dollars }\end{array} \\ \text { ARD - Atmospheric Resid Desul } & 40000 & 122.0 \\ \text { RDS - Residuum Desulfurization } & 40000 & 151.0 \\ & & \\ \text { HCV- Residuum Hydrocracking } & 40000 & 265.0 \\ \text { FDS - FCC Feed Desulfurization } & 40000 & 100.0 \\ & & \\ \text { HCL- Mild Hydrocracking } & 40000 & 124.0 \\ \text { HCR- Moderate to Severe Hydrocracking } & 40000 & 195.0 \\ \text { HCU- New Generation Technology* } & 40000 & 242.0 \\ \text { MAK- Exxon Mobil Makfining } & 40000 & 184.0 \\ & & 160.0 \\ \text { FCC Catalytic Cracker } & 40000 & \\ & & 43.3 * * \\ \text { SYD - Synsat for Diesel } & 30000 & 38.5 \\ \text { HD1 - First Stage Deep Desulfurization } & 30000 & 12.3 \\ \text { HS2 - Second Stage Deep Desulfurization } & 30000 & 32.1 \\ \text { HD2 - Stage 2- Dearomatization } & 30000 & \end{array}$


EnSys Energy \& Systems, Inc.

$\begin{array}{lll}\text { DDS - Low Severity Distillate Desulfur } & 30000 & 30.3 \\ & & \\ \text { *** - Advanced Desulfurization Type 1 } & 30000 & 36.0 \\ \text { *** - Advanced Desulfurization Type 2 } & 30000 & 32.2 \\ \text { BID Diesel Bio Desulfurization } & 30000 & 35.0 \\ & \text { MMSCFD } & \text { million dollars } \\ & 60 & 53.4 \\ \text { H2P Natural gas Steam Reforming } & 40 & 10.0 \\ \text { HPM Hydrogen Membrane Purification } & & 9.5 \\ \text { PSA Hydrogen Purif - Swing Adsorption * } & 40 & \\ \text { * New process unit additions to RYM } & \\ \text { ** Capital cost for SYD is high because based on 32\% hydro-dearomatization versus } 17 \% \text { for } \\ \text { HD1 unit } \\ \text { The above hydrogen purification units HPM and PSA contain a 1.33 CAP row entry to reflect } \\ \text { 25\% downtime for regeneration. A scale factor of 0.9 is recommended given the modular nature } \\ \text { of the units. }\end{array}$

\section{$\underline{\text { V. Distillate Properties Review and Corrections }}$}

All distillate stream properties were reviewed and corrected where necessary. The attached Excel spreadsheet summarizes the effort and results. The properties review included sulfur, aromatics specific/API gravity, cetane number and kerosene smoke point (where required). The RYM model properties updates are shown in red, predominantly for aromatics content and cetane number. The Synsat unit aromatics contents were adjusted to a less severe operation consistent with hydrogen usage and all cetane number values were checked against an API correlation supplied by Exxon Mobil. A few API gravity corrections were also made.

Note, the SynSat unit effectively equates to the LSD unit and the HD1/HS2/HD2 complex to the ULSD processes.

\section{Hydrogen Plant Revamp/Debottleneck}

The natural gas fed steam reforming plant hydrogen plant represented in Table H2P was updated for natural gas and utilities consumptions to reflect current Foster Wheeler performance. Sources are Meyers, Second Edition, page 6.61 and Technip NPRA paper AM-01-19, page 9, Table 2. The latter was for an Air Products and Chemicals plant and involved a comparison of the variable cost of hydrogen production with the updated Table H2P values. The ISBL capital investment cost is from Gary and Handwerk, $4^{\text {th }}$ Edition, page 264. The overall result is to lower the cost of producing hydrogen versus the previous RYM representation. 


\section{EnSys Energy \& Systems, Inc.}

Three NPRA papers were reviewed for hydrogen plant revamp potential - AM-01-19, Air Products and Chemicals/Tecnip, AM-01-27, Haldor Topsoe and AM-01-27, Foster Wheeler, AM-01-36. It is estimated that a 30\% increase in capacity can be achieved for $25 \%$ of the ISBL and $10 \%$ of the OSBL investment costs, with a marginal increase in energy efficiency of $8 \%$.

There are two other primary options aside from de-bottlenecking:

Retirement and replacement - According to Air Products, during the period 1991 through 2000, refiners added approximately 300 MMSCFD of new capacity and retired 130 MMSCFD. Although Foster Wheeler states that the tighter new plant designs offer less revamp potential than the older plants, on the other hand they note many of the older plants have been revamped at least once. From the above figure for outright hydrogen plant retirement, the revamp potential is definitely limited.

Across the fence hydrogen purchase. During the same 1991-2000 period, industrial gas companies added 850 MMSCFD of capacity for sale to refiners. This option is becoming attractive to refiners because they tend to avoid the (now near catastrophic) consequences of a sudden hydrogen plant shutdown, they avoid expending investment capital and they provide increased refinery operations flexibility.

The modeling challenges for representing the important cost of hydrogen appears to lie less in estimating the cost of the various supply options than in estimating their likely adoption.

\section{Hydrogen Purification and Recovery}

Two units have been modeled - the existing HPM unit for membrane purification and a new RYM Table PSA to represent pressure swing absorption. The latter is gaining ground since higher purity hydrogen is produced at a higher pressure.

Table HPM representing hydrogen membrane purification is based on data published in Hydrocarbon Processing, April 2000, p72 for the Medal process and on UOP NPRA Paper AM99-08. Investment cost is also based on European Union data available from the Internet.

Table PSA investment cost for swing absorption is based on investment cost based on comparisons from Gary and Handwerk, Fourth Edition, Meyers, Second Edition, World Refining, October 2001 and current Oil and Gas Journal Construction Survey costs.

If both units are allowed to ride free, the validity of the relative selection may be in question. The higher purity of the PSA hydrogen facilitates middle distillate desulfurization to lower sulfur levels (one reason for its selection), but we do not represent different hydrogen purity levels in RYM. A disadvantage of the membrane process is the need for hydrogen recompression and we do not represent different hydrogen pressure levels in RYM, although we can make an 


\section{EnSys Energy \& Systems, Inc.}

adjustment to the HPM investment cost. Also, total hydrogen purification may exceed reasonable values since site specific limitations are a reality.

It is proposed to insert Table POL constraints to limit both total hydrogen purification capacity and PSA versus HPM - current Oil and Gas Journal Refining Survey data indicates that hydrogen purification capacity accounts for 38\% of hydrogen plant production and the survey shows PSA to dominate over HPM and cryogenic separation, which is relatively minor.

\section{Middle Distillate Deep Desulfurization and Aromatics Saturation}

\section{A. Existing RYM Process Units}

Building on the recent model development work for API, two NPRA Papers were reviewed in depth: AM-01-22 by Mustang Engineers and Constructors, Inc and AM-01-28 by Haldor Topsoe and San Joaquin Refining Company.

These papers address hydrotreater revamp potential and the incremental steps required to achieve less than $10 \mathrm{ppm}$ product sulfur. The Mustang paper gives ULSD revamp cost estimates. The requirements for aromatics saturation are also addressed in these papers.

The two papers essentially agree on the revamp steps required to achieve ULSD:

the addition of a second reactor or a new second stage hydrotreater to lower space velocity by providing increased catalyst volume

more active catalyst directed at desulfurizing sterically hindered sulfur compounds (look at NiMo versus CoMo).

higher hydrogen purity from hydrogen purification

scrubbing of the recycle gas to provide higher hydrogen purity

higher reactor pressure to be considered in addition to the above to increase the hydrogen partial pressure in the reactor.

The reactor internals must also be examined since a one percent bypassing of the feed can contribute 50 to $150 \mathrm{ppm}$ sulfur content to the diesel product.

In order to saturate aromatics to the 4 weight percent level, increase feed cetane by 10 numbers and API by 6 , a second high pressure noble catalyst stage is required, along with an intermediate stripper column to remove dissolved $\mathrm{H} 2 \mathrm{~S}$ and $\mathrm{NH} 3$ from the first stage effluent 


\section{EnSys Energy \& Systems, Inc.}

\section{$\underline{\text { Revamp Potential }}$}

Both NPRA papers state that units built since the early 1990s may be revamped according to the above scheme and that additional revamps of the type above to already revamped units may not be feasible. To revamp an earlier generation hydrotreater (1970's and 1980's) may require up to three times the catalyst volume, as opposed to an increase of 100 percent in the catalyst volume for the post 1990 units.

\section{$\underline{\text { Revamp Cost }}$}

For a 30,000 BPD unit with 1/3 LCO feed, Mustang estimates 8.6 million dollars for the above sequence for a $100 \%$ catalyst volume increase. This is an ISBL figure with an accuracy stated of 20 percent up or down. An offsites cost range of 30-100 percent onsite investment is quoted. The Mustang paper indicates a 0.9 million dollar ISBL increase in going from a 50 to 100 percent catalyst volume increase. Applying economies of scale, this provides a basis for estimating the revamp cost of a pre-1990s hydrotreater requiring three times the catalyst volume.

\section{B. New RYM Distillate Desulfurization Process Units}

Two new units were selected and added to represent prospective distillate desulfurization processes under development:

\section{Advanced Desulfurization Type 1}

This process is claimed to reduce product sulfur to $10 \mathrm{ppm}$ and lower using a sorption process. The process is in the pilot plant stage with the possibility of commercialization to follow in the short term. (Commercial gasoline units are in operation and the licensor maintains there is little difficulty in extending the process to diesel.) Both virgin and LCO feed streams are represented with low hydrogen consumption in the 40 to $170 \mathrm{SCF} / \mathrm{bbl}$ range. Cetane improvement and aromatics reduction does not occur to any marked degree. This process is described in *****, giving process data and estimated variable and investment costs.

\section{Advanced Desulfurization Type 2}

This process claims to reduce the sulfur content of virgin distillate and LCO to the 10-20 ppm sulfur content level. There is no hydrogen consumption reported, however there is a 9 to 15 cetane number increase claimed, along with a reduction in aromatics content. The process claims a novel approach, using kinetic ultrasonic waves targeted at sulfur atoms to raise local pressures to 10,000 psi accompanied by hydrogen transfer from water. The sulfones that are formed in the

process are extracted with a solvent. This process is described in *****, giving process data and 


\section{EnSys Energy \& Systems, Inc.}

estimated variable and investment costs. . After earlier promising tests, questions remain about the consistent performance of the process, with the need for more R\&D indicated.

The Unipure ASR-2 Diesel Desulfurization process is described in NPRA paper AM-01-10. This process is also based on employing a liquid catalyst and the removal of sulfur via sulfones, but like $* * * * *$ does not claim significant cetane improvement or dearomatization. While using a different process than $* * * * *$, the Unipure ASR-2 process description gives similar estimates for product sulfur content and investment and operating costs. Therefore this duplicative process was not modeled. However, future developments will be tracked - the process is in the pilot plant stage and a commercial application is projected. The process may be modelled in the future.

The Englehard-Washington REDAR process for upgrading light cycle oil was also not modeled at this point. The hydrocracker and deep desulfurization/dearomatization process units described above provide ample avenues for up grading light cycle oil. NPRA paper AM-01-23 describes the REDAR process and while it may produce a lower sulfur than HD2 (4 versus $10 \mathrm{ppm}$ ), it is still in the pilot plant stage. The process may be modelled in the future.

Daniel N. Dunbar

Martin R. Tallett 



\section{APPENDIX I-C}

API COMMENTS ON RESEARCH PROPOSAL

"ESTIMATING IMPACTS OF DIESEL FUEL REFORMULATION WITH VECTOR-BASED BLENDING" 



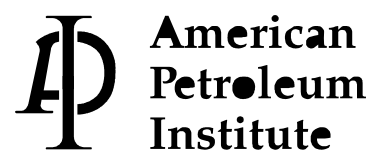

\begin{abstract}
API Economics Work Group and Diesel Issues Subcommittee: Thanks very much for your helpful comments on the Scenario Document. DOE responses are inserted in the text. As noted, we welcome further guidance. Barry McNutt and Jerry Hadder
\end{abstract}

Date: $\quad$ May 21, 2002

To: $\quad$ Barry McNutt, U.S. Department of Energy, Office of Policy and International Affairs; Jerry Hadder, Oak Ridge National Laboratory

From: $\quad$ API Economics Work Group and Diesel Issues Subcommittee

Re: $\quad$ Comments on Research Proposal "Estimating Impacts of Diesel Fuel Reformulation with Vector-Based Blending"

The API Economics Work Group and the Diesel Issues Subcommittee appreciate the opportunity to comment on the DOE research proposal entitled "Estimating Impacts of Diesel Fuel Reformulation with Vector-Based Blending". As you know, we support the high quality research on the economics of fuel regulation that the DOE undertakes, and value DOE's input on some of the similar efforts that API undertakes. During this process of constructive engagement, there are times when DOE may disagree with an assumption or study premise utilized in an API study, or at times we may disagree on a particular DOE study's timing and/or premises. It is in this spirit of constructive engagement that the following comments are offered for your consideration. We also suggest that a meeting with you to discuss the DOE proposal may improve the mutual understanding of the overall effort and result in an improved work product. In this light, the API Economics Work Group is scheduled to meet in Washington on June 18 and, if you concur, would be available to meet with you on that day. We will follow up with you shortly regarding this potential meeting.

\section{Proposed DOE Study is Premature: Definitive Studies of Emission Benefits of RFD Needed First}

The proposed study is based on the initial premise that controls on diesel fuel quality (beyond the reduction of sulfur) will be necessary "to enable either lower in-use emissions, new vehicle emissions control system performance or vehicle operating characteristics..." This premise is both unfounded and untested [See DOE Reply 1]. There have not been any definitive studies that have assessed the need for (or evaluated the costs and benefits of) such controls. In particular, we are aware of no data that reliably captures the impact of changes in fuel parameters on emissions from the new engines and after-treatment 
technology that manufacturers plan to introduce either in compliance with the Tier 2 standards for light-duty vehicles and light-duty trucks or in response to the 2004/2007 standards for heavy-duty engines. In fact, as API noted in recent comments pertaining to the EPA draft diesel fuel effects model, data on the impacts of fuel properties on emissions from model year 1996 and newer diesel trucks are extremely sparse. Furthermore, there is insufficient information in the existing literature to adequately separate the emissions effects of individual changes in fuel properties. [See DOE Reply 2] Taken together, this makes projections of emissions (and associated benefits) for 2010 and beyond highly uncertain. Until these technologies become available and are adequately tested, there is no way to determine benefits from further highway diesel reformulation past the 15 ppm sulfur cap requirement.

It also is important to note that even if diesel fuel reformulation does provide some quantifiable benefit in the short term (i.e., 2010) by reducing emissions from the current in-use fleet, these benefits will decline over time, particularly in relation to those derived from the introduction of advanced technology to meet more stringent emissions standards [See DOE Reply 3]. For example, the EPA has estimated that the 2007 NOx standard for new heavy-duty diesel engines will ultimately result in about a 90\% reduction in in-use heavy-duty diesel vehicle fleet emissions over the next 30 years due to fleet turnover and the expanded penetration of cleaner technologies into the fleet. Clearly, when measured against a backdrop of diminishing in-use emissions in the future, any benefits from fuel reformulation will become increasingly marginal. This suggests that improvements in vehicle engine and after-treatment technology are likely to be significantly more cost-effective than fuel reformulation. It may also pose less of a risk to fuel supply than fuel reformulation.

Until these assessments have been made and validated, it is difficult to justify a study that focuses on the timing and interaction of additional diesel fuel quality controls beyond sulfur reductions with existing diesel regulations. Nor, in our opinion, could the results of such a study be considered reliable given the deficiencies in existing data as explained above.

\section{DOE Reply}

1. The premise document should not be interpreted as a DOE proposal or support for changes in diesel fuel specifications. DOE's interest is transportation fuel supply. Given support of the World Wide Fuel Charter by global vehicle and manufacturing associations, DOE needs to understand the potential Charter implications for transportation fuel supply. 
2. DOE understands that there are data limitations. Recognizing the difficulties in separating the emissions effects of individual changes in fuel properties, DOE developed the eigenfuel approach, which correctly separates the emissions effects of changes in groups of properties.

3. An analysis based on existing data is appropriate for projecting emission changes in the existing on-road fleet of conventional engines. The analysis will be applied on a "percentage or relative basis" in estimating emission changes from newer engines, so that the emissions effect possible through fuel reformulation will decline as the baseline emission levels of engines decline.

\section{Model of Emission Reductions to Assess Refining Flexibility has not been adequately Defined or Reviewed}

Part of DOE's assessment will be to determine if refining flexibility can be provided by regulating via the establishment of an "emissions performance" standard versus specific "parameter control". Any conclusions regarding this flexibility will be highly dependent upon the emissions model utilized to perform the evaluation. Yet, the DOE proposal provides inadequate detail concerning the methodology, assumptions and approach that is intended for use in this regard [See DOE Reply 4].

The DOE proposal appears to suggest that conventional analytical tools that correlate changes in diesel fuel properties with exhaust emissions (similar to the draft model recently proposed by EPA) are fraught with statistical issues relating to the correlation of individual fuel properties. We agree with this assessment. In fact, API submitted extensive public comments on the draft EPA diesel fuel property effects model. Our primary concern was that severe limitations in the emissions database underlying the model (particularly relating to fuel impacts for 1996 and newer heavy-duty diesel engines) made it impossible to adequately separate individual fuel property effects no matter what statistical procedure was used let alone reliably predict fuel emission impacts in 2010. In addition, API was concerned that the draft EPA model over-predicted future fuel effects because it relied on data from older engines that were not representative of the future fleet of heavy-duty highway diesel vehicles.

The DOE proposal indicates that an "eigenfuels methodology" will be used to estimate emissions in terms of independent vector variables. However, the proposed DOE approach has not been adequately peer reviewed and it is unclear that it will overcome fundamental limitations in the underlying database of available studies [See DOE Reply 5]. In fact, the EPA assessed the DOE eigenfuels methodology using the database that supported its draft model fuel effects NOx model and concluded that the DOE approach "... did not assist in eliminating unimportant fuel terms, and therefore did not appear to offer an advantage over more traditional regression analyses" [See DOE Reply 6].

Finally, while by no means experts on the creation and use of eigenfuels, we are not convinced that such an approach can adequately represent unique technologies (such as adsorption) or 
feeds (syncrude, GTL, Bio, etc.). We question the proclamation that Eigenfuels provide an "unambiguous and theoretically correct method for cost-effective blending".

DOE Reply

4. The eigenfuel methodology is described in detail in our report provided to Mike Leister (PCR+in Diesel Fuels and Emissions Research). A copy of the report will be mailed to Art Weise. Please advise us if additional copies are needed.

5. The following peer-reviewed reports have been published:

H.T. McAdams, R.W. Crawford, G.R. Hadder. 2000a. A Vector Approach to Regression Analysis and Its Application to Heavy-Duty Diesel Emissions, SAE 200001-1961, Society of Automotive Engineers International Spring Fuels \& Lubricants Meeting \& Exposition, Paris, France, June 19-22.

H.T. McAdams, R.W. Crawford, G.R. Hadder. 2000b. A Vector Approach to Regression Analysis and Its Application to Heavy-Duty Diesel Emissions, ORNL/TM2000/5, Oak Ridge National Laboratory, Oak Ridge, TN 37831, November.

McAdams, H.T., R.W. Crawford, G.R. Hadder. 2002. PCR+in Diesel Fuels and Emissions Research, ORNL/TM-2002-16, Oak Ridge National Laboratory, Oak Ridge, TN 37831, Mar

In addition, Robert Crawford presented "Issues \& Concerns in Development of Diesel Fuel Emissions Models" at the EPA Diesel Fuel Effects Analysis Workshop. Crawford presented "Multi-Property Vector Approach to Fuels and Emissions Analysis" at the DOE Office of Transportation Technologies CIDI Review. We also distributed, with request for comments from several industry contacts, "Eigenvectors of Commercial Diesel Fuels."

6. EPA made only a limited effort in assessing eigenfuels as an alternative method during their 2001 study on diesel emissions, and they ended their effort with an incorrect application of the eigenfuel technique for screening out unimportant fuel terms.

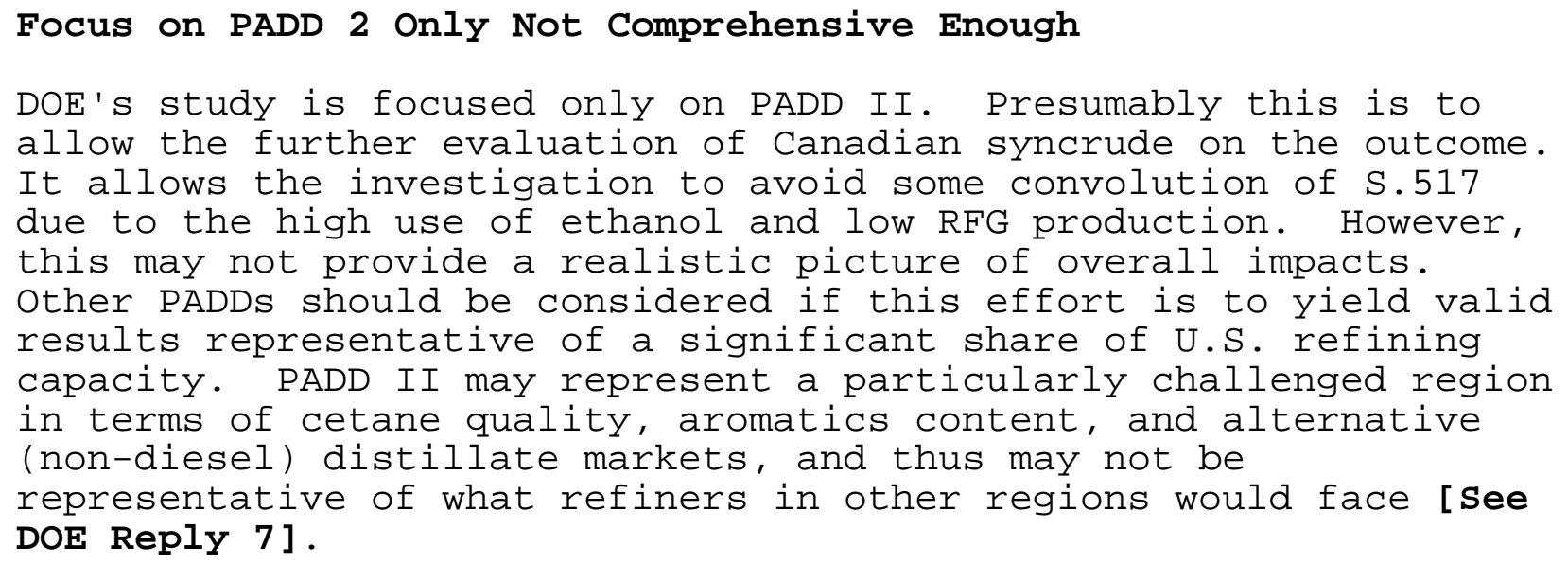


7. DOE did not intend to imply that PADD 2 was selected as representative of the nation. Future work may examine other PADDs.

\begin{abstract}
Assumption that 500ppm $S$ non-road fuel will be $360 \mathrm{ppm} S$ actual Open to Question

It is not at all clear that a 500 ppm $S$ non-road fuel will be 360 ppm $S$ actual. Logic suggests that LSD will not have as much sulfur "giveaway" in a post ULSD world - particularly if high sulfur non-road goes away. LSD will be made from the harder to treat diesel streams while virgin streams that currently go to LSD will be used for ULSD. While these factors might suggest higher actual sulfur levels well above $360 \mathrm{ppm}$, the big unknown is the impact that downgraded (contaminated/interfaces) ULSD will have? High levels of downgrading could tend to bring the actual sulfur level back down some.

In addition, DOE's reference case assumes that all non-road diesel fuel (inclusive of home heating oil) will be at $500 \mathrm{ppm}$ sulfur or less in 2010. Imposing such a constraint on the reference case may distort the basis such that additional parameter controls on cetane and aromatics are less than otherwise would have been the case had a more realistic reference case been assumed [See DOE Reply 8].
\end{abstract}

\title{
DOE Reply
}

8. DOE welcomes specific guidance from API on these questions.

\section{Assumption that the Chicago/Milwaukee VOC offset goes away Open to Question}

We are not aware of the reason for this assumption, and would appreciate further explanation as to the basis for it [See DoE Reply 9]

\section{DOE Reply}

9. The VOC assumption is made in a post-Senate Energy Bill context. With an end to the oxygen requirement, more stringent $\mathrm{VOC}$ requirements, etc., DOE believes that the Scenario Document makes a plausible assumption for the VOC offset.

DOE's Table 1 should exclude Sweden Class 1

DOE's Table 1 should exclude Sweden Class 1 as this is more of a kerosine than a diesel fuel [See DOE Reply 10].

\section{DOE Reply}

10. For clarity, the table will be so-revised. 
Use of Ratio-Free RYM Model can yield Unrealistic Results

The API Econ WG has earlier questioned the use of the ratio-free RYM model as unrealistic yielding results, i.e. is not representative of the lower end of the range [See DoE Reply 11].

\section{DOE Reply}

11. The uncomposited updated refinery model will be used because the composited model has not yet been updated. In its case studies (supporting DOE comments in response to the EPA's May 16, 2000 Notice of Proposed Rulemaking on Heavy-Duty Engine and Vehicle Emission Standards and Highway Diesel Fuel Sulfur Control), EnSys observed that the uncomposited model allows the analysis to fully differentiate between deep hydrotreating of easy versus difficult streams.

Updated RYM Model not Transparent

The RYM has undergone some updates that have not been widely reviewed. It would be helpful if DOE would present details of the model updates for our better understanding of capabilities and assumptions [See DOE Reply 12].

DOE Reply

12. EnSys' progress reports on the updating tasks are in Appendices I-A and I-B.

Assumption to hold On-Road Diesel-Equivalent MPG Constant across all cases Open to Question

DOE plans to hold on-road diesel-equivalent mpg constant across all cases. This may not be realistic as reformulation through hydrogenation will effect energy density.

In addition, under the study methodology it is stated that cost changes will not account for the consumer fuel economy effect. At a minimum, refinery out-turns should be adjusted to accommodate lower energy density resulting from reformulation

[See DOE Reply 13].

\section{DOE Reply}

13. The Scenario Document statement on constant mpg means that refinery out-turns will be adjusted to accommodate consumer demand.

If incremental RFG is from refineries not currently producing RFG, then the standard for that RFG production is 25.3 percent toxics reduction and not 26.7 .

EPA's MSAT rule at 80.855 (b) (1) (ii) has a default toxics standard for RFG as 26.71\% reduction; this is applicable if a refinery did 
not produce RFG between 1998 and 2000. EPA has admitted that this value was calculated incorrectly and should be 25.3\%. EPA has not officially corrected its mistake because it will issue a revised value based on three years (1998-2000), not just the two years (1998-1999). The 26.71 was meant as an interim value until EPA could review/include data for 2000 .

The DOE proposal states on p.7: "RFG and CG will satisfy the toxics anti-backsliding rulemaking of 2001 (EPA 2001).

Incremental RFG will satisfy one of two possible toxics standards. If incremental RFG is produced by refineries currently producing RFG, then the standard for incremental production is 21.5 percent toxics reduction. If incremental RFG is from refineries not currently producing RFG, then the standard for incremental production is 26.7 percent toxics reduction."

The last sentence of this paragraph should read: "If incremental RFG is from refineries not currently producing RFG, then the standard for that RFG production is 25.3 percent toxics reduction" [See DOE Reply 14].

DOE Reply

14. The toxics specification for incremental RFG will be so-revised.

Other Comments/Questions:

Grade split document for gasoline by Weihrauch 2001 should be reviewed [See DOE Reply 15].

DOE Reply

15. The Weirauch data imply Class splits, rather than grade splits. The document will be mailed to Art Weise.

How are Jet and Kero handled within the study. Are they also at the 500 ppm max? [See DOE Reply 16].

DOE Reply

16. Jet $A$ and kerosene are in a pool assumed to have Jet $A$ quality. The target sulfur content $(863 \mathrm{ppm})$ is based on the 1996 American Petroleum Institute/National Petroleum Refiners Association Survey of Refining Operations and Product Quality. DOE welcomes alternative guidance from API on this question. 

APPENDIX I-D

API COMMENTS ON DOE RFD STUDY 



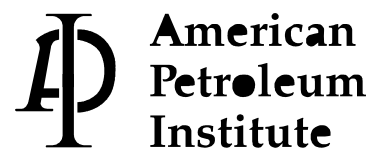

Date: September 16, 2002

To: $\quad$ Barry McNutt, U.S. Department of Energy, Office of Policy and International Affairs; Jerry Hadder, Oak Ridge National Laboratory

From: API Economics Work Group and Diesel Issues Subcommittee

Re: $\quad$ Comments on DOE RFD study

Art:

Thanks very much for API comments on the DOE RFD study. Please see bold text for our reply. If additional information would be helpful, please call me at (865) 483-9287.

Jerry Hadder

Oak Ridge National Laboratory

The API Economics Work Group and the Diesel Issues Subcommittee appreciate the opportunity to comment on the DOE RFD study. Below are comments based on the DOE output dated August 19, 2002. We apologize for the delayed response but between holidays and a myriad of other issues, plates here have been pretty full. Thanks again for keeping us up-to-date, and we would welcome the opportunity for further input as the study continues.

\section{DOE Study is Premature: Reliable Emissions Data Needed First}

The reliability of the NOx and $\mathrm{PM}$ results remain open to question. It appears that the sulfur/cetane/aromatics control case is driving PM and NOx to very high performance values. This suggests serious problems with the underlying data. This reflects the fact that no data exist that reliably capture the impact of changes in fuel parameters on emissions from the new engines and after-treatment technology that manufacturers plan to introduce either in compliance with the Tier 2 standards for light-duty vehicles and light-duty trucks or in response to the 2004/2007 standards for heavy-duty engines.

We understand DOE's interest in assessing the economic and supply impacts of fuel reformulation given global vehicle manufacturing support of the World Wide Fuel Charter, but we continue to 


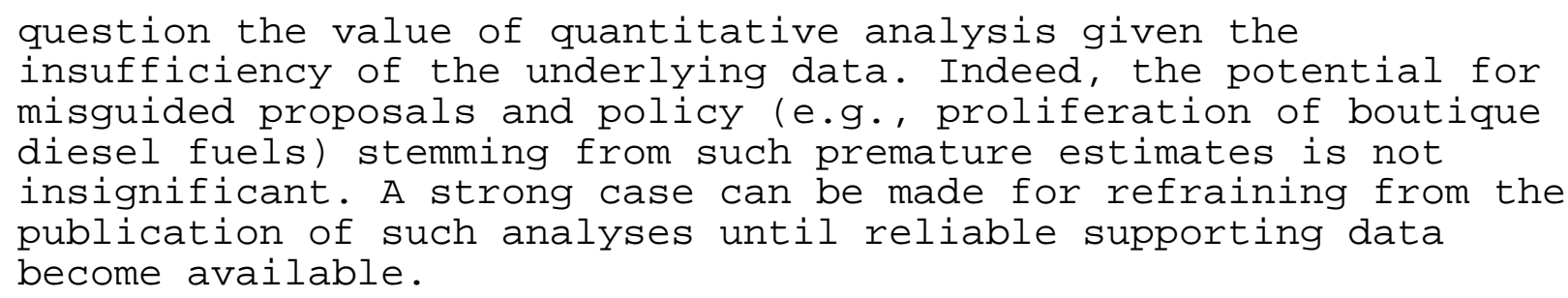

The DOE RFD study should not be viewed as a prelude for DOE policy proposals or support for changes in diesel fuel specifications. DOE is studying diesel fuel reformulation because of the Department's interest in transportation fuel supply. Given support of the World Wide Fuel Charter by global vehicle and manufacturing associations, DOE needs to understand the potential Charter implications for transportation fuel supply. DOE also needs to understand the implications of API's view that "it would be preferable to set more general in-use performance specifications for fuels that allow flexibility in formulation as opposed to arbitrary limitations on major fuel components that yield limited benefits at high cost" (Murphy letter to Hodac et al, September 3, 2002).

With regard to emissions data, API raised the following concerns in our June 18 meeting. DOE is taking the action noted:

1. Data are insufficient and inappropriate for estimation of emissions model. Action: Cite concern in final report.

2. It is inappropriate to assume that percent reductions of emissions in the current fleet will apply equally to future engine-aftertreatment systems. Action: Cite concern in final report. Our premise will be retained because we nor API Committee can suggest a better alternate premise.

3. Diesel engines with EGR may have a NOx response to cetane that is directionally different from the data used in eigenfuel-based model development. Action: Robert Crawford will test performance of eigenfuel-based emissions model on EGR data that were not used in emissions model estimation. We will acknowledge concern in final report; include Crawford's findings; include comments from EPA420-S-02-012 (EPA claims "no discernable effect" of cetane on NOx emissions from EGR equipped engines, whereas API said cetane increases "increased NOx".")

DOE understands that there are data limitations and uncertainties and will consider updates of the RFD study as data uncertainties are resolved. Nevertheless, DOE will complete and publish the present study in order to develop an understanding of the directional implications of RFD on transportation fuel supply.

Characterization of New Technology 
DOE inappropriately is referencing $* \star \star \star *$ in its text. This is not an $\star \star \star \star \star$ study but rather an advanced technology study

considering technologies that have high capability to selectively remove sulfur without affecting other properties. DOE should remove its references to $\star \star \star \star \star *$ utilization and refer to advance technologies or maybe more appropriately, to low H2 consumption technologies that selectively remove sulfur.

DOE will do so.

Market Penetration of New Technology

The DOE study assumes a 15\% market penetration of new technology in base case 2. What is the basis for this assumption? While this market penetration rate does not seem unreasonable, it will be highly dependent upon the technology premises imposed. Presumably this new technology includes greater sulfur selectivity of conventional HDT catalyst and greater reactivity or other advanced technology besides just $\star \star \star \star * *$. It is not clear what technology premises DOE is considering but the assumptions should be explicitly laid out in the report.

Technology premises will be detailed in the report by including the Ensys progress reports on the ORNL-RYM technology updating tasks. These progress reports were included as response No. 12 to the API letter of May 21, 2002. The market penetration rates are assumptions that we believe (as you state) are not unreasonable. We also received independent consultation that the market penetration assumption is not unreasonable.

Cost Impacts of New Technology

The DOE results indicate a significant cost reduction attributed to new technology in base case 2 , i.e., $5.2 \mathrm{cpg}$ vs $7.9 \mathrm{cpg}$ if it were all hydrotreating. Based on a presentation at the July 2002

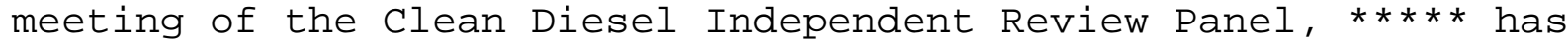
on-site capital costs similar to hydrotreating, but gains its savings through reduced operational costs and offsite requirements, e.g.,hydrogen. Hence, does it make sense that a $15 \%$ penetration of this technology could reduce overall ULSD cost by a third? Or stated differently, the absence of the 15\% penetration increases ULSD cost by half?

Backing down the steep end of the cost curve will have a substantial effect on reducing average costs. For example, the DOE results may be consistent with the Baker \& O'Brien ULSD Supply and Cost curve from NPRA Paper AM-01-32, page 20. For the purposes of illustration only, re-scaling the numbers in the Baker \& $O^{\prime}$ Brien curve to approximately match the DOE study: $7.9 \mathrm{cpg}$ falls to $5.7 \mathrm{cpg}$ at 85 percent volume. With new technology costs at $3.7 \mathrm{cpg}:(5.7 \mathrm{cpg}) * .85+(3.7 \mathrm{cpg}) *(0.15)=5.4 \mathrm{cpg}$ for the overall average. 
Inadequate Basis for DOE Preliminary Finding related to New Technology

There is inadequate basis for the preliminary DOE finding that "newer technologies contribute to increased costs of sequential investment pathway compared to parallel investment pathway". This conclusion is dependant upon the assumed rate of new technology adoption, a parameter with high uncertainty. At the least, qualification of the DOE finding is necessary. It is suggested that additional sensitivity cases in 2.1 and 2.2 would be needed to further investigate the robustness of this conclusion. Also, differentials between parallel and sequential investments are driven by factors other than new technology (configuration, size, pressure, timing, H2 capacity, etc.). It is not clear the extent to which the model can capture the cost savings associated with parallel investment vs sequential investment. This should be clearly laid out and discussed in the report.

For the purposes of understanding directional impacts of new technology, DOE has assumed a technology adoption rate which does not seem unreasonable (see above discussion of "Market Penetration of New Technology."). We do not believe there is a need, at this time, to perform additional sensitivity studies. However, we will include your letter in our report, as a record of your concern. We understand that the differentials between parallel and sequential investments are driven by a set of complex inter-related factors. We will discuss some of these factors in our report.

DOE including Sulfur Reductions in Home Heating Oil?

Based on the footnote on page 4 (labeled "Case Study Summary") of its output, DOE appears to be including sulfur reductions for home heating oil in its modeling. We are unsure of the reason for this as we are not aware of any discussions to date to regulate home heating oil (HHO), and such regulation does not fall under the purview of the EPA. We suggest that the cases be rerun without desulfurization of $\mathrm{HHO}$ to $360 \mathrm{ppm}$.

Heating oil sulfur was specified to be consistent with prior EnSys studies used as the basis for DOE comment on EPA proposals for ULSD. Given heating oil's relatively small volume (less than 10 percent of the total off-road production volume) in our study, and uncertainty about future requirements of pipelines and end-use customers, DOE will not rerun the cases at this time. 


\title{
PART II \\ DIESEL FUEL EMISSIONS MODELS \\ FOR THE ORNL REFINERY YIELD MODEL
}

July 2002

\author{
Prepared by: \\ RWCrawford Energy Systems \\ Tucson Arizona
}

Prepared for:

Oak Ridge National Laboratory

Oak Ridge, Tennessee

managed by

UT-Battelle, LLC

for the

U.S. DEPARTMENT OF ENERGY

under contract DE-AC05-00OR22725 



\section{II-1. INTRODUCTION}

\section{II-1.1 BACKGROUND}

The potential for reformulating diesel fuel to reduce emissions is of considerable current interest. In 1993 the State of California established a reformulation program with emissions performance standards for diesel fuel in an effort to reduce emissions of nitrogen oxides $\left(\mathrm{NO}_{\mathrm{x}}\right)$, particulate matter $(\mathrm{PM})$ and air toxics. More recently, the State of Texas proposed a similar diesel fuel program, and other states have considered such programs.

The attractiveness of diesel fuel reformulation to state authorities stems from the potential for achieving emissions reductions from the in-use vehicle fleet - predominantly heavy-duty diesel (HDD) engines - in a short to intermediate time frame. Beginning in this period and continuing over a longer time frame, new advanced-technology diesel engines with much lower exhaust emission levels will enter the fleet and substantially reduce the HDD contribution to fleetwide emissions. Other parties, including engine and vehicle manufacturers, may have interest in diesel fuel reformulation (beyond sulfur reductions) to enable new emission control technologies or improve vehicle operating characteristics.

In response to the interest in diesel fuel reformulation, the U.S. Environmental Protection Agency (EPA) initiated a research effort to relate diesel fuel characteristics to HDD emissions. Relying on the compilation of emissions test data already published in the technical literature, the agency developed statistical models for exhaust emissions as functions of fuel properties such as aromatics content, specific gravity, and cetane number. The EPA work is summarized in two publications (EPA 2001, SwRI 2001) and was presented at a public workshop in August 2001. Although recognized for contributions to the understanding of these issues, the results of the EPA effort were greeted with considerable discussion and some controversy in terms of data adequacy, statistical methodology, selection of variables, and model predictions. EPA subsequently concluded the work without adopting an approved statistical model of emissions for regulatory use.

Oak Ridge National Laboratory (ORNL) has been involved in the analysis of diesel fuel and emissions issues since 1998 on behalf of the U.S. Department of Energy (DOE). This work is been motivated by the understanding that diesel fuel reformulation could have substantial impacts on U.S. fuel supply and should be undertaken only on the most reliable technical assessment of benefits and costs. The ORNL work has involved refinery impact studies, emissions test data analysis, and the development of improved statistical methodologies for assessing the diesel fuel / emissions relationship.

One outcome of this work has been the development of the eigenfuel methodology for diesel fuels and emissions research as an alternative to the conventional research paradigm in which:

- Experimental fuels are blended in an effort to vary selected diesel fuel properties in isolation from each other, and

- Stepwise regression is used as the primary technique to select among competing statistical models of the resulting emissions test data.

Diesel fuels are strongly affected by naturally-occurring relationships among the individual fuel properties, as are all diesel fuel and emissions data in which the relationships have not been artificially eliminated. In this realm, ORNL has concluded that the influential factors for emission are better described by eigenfuels - vector variables representing combinations of the fuel properties. The eigenfuel methodology and its 
application in diesel fuels and emissions research are described more fully in two recent ORNL publications (McAdams 2000a,b and McAdams 2002).

\section{II-1.2 PURPOSE OF THIS WORK}

During 2002 ORNL will conduct a series of assessments of the impacts of a reformulated diesel fuel requirement on refinery investment and operating costs using the Refinery Yield Model (ORNL-RYM $\left.{ }_{2002}\right)$ as updated in 2002. Case studies will be conducted as required by the DOE Offices of Policy and International Affairs, Energy Efficiency and Renewable Energy, and Fossil Energy to focus on the refinery implications of issues such as the following:

- A national on-road diesel fuel reformulation requirement

- The interaction of such a requirement with the existing 2006 Ultra-Low Sulfur Diesel requirement

- How diesel reformulation is specified in terms of emissions performance or specific levels for fuel properties

- Changes to off-road diesel fuel quality

- Changes to gasoline requirements

- Greater use of Canadian synthetic crude oils.

The work described in this report was designed to support the planned case studies through the development of statistical models that can be used within RYM to estimate the emissions impacts of finished diesel fuels. The work and the resulting statistical models should be understood as an analytical tool to help advance the technical understanding of the diesel fuel / emissions relationship and to support the assessment of how diesel fuel reformulation could impact the refining sector. It should not be taken as a conclusive assessment of how diesel fuel characteristics influence emissions in HDD engines, because R\&D in this area remains at a comparatively early stage. Indeed, the structure and coefficients of the models may change over time as new data and improved methodology become available.

\section{II-1.3 SUMMARY}

Statistical models of the relationship between HDD engine emissions and the physical, chemical, and compositional properties of diesel fuels are developed using the ORNL eigenfuel methodology. These models predict the fractional (percentage) change in emissions for a fuel relative to the emissions level for the average diesel fuel currently sold in the U.S. For the first time, nonlinear terms have been incorporated in the eigenfuel methodology, including both quadratic $\left(\mathrm{X}^{2}\right)$ terms representing saturation phenomenon and interaction terms among properties.

The database compiled by EPA in its 2001 work has been employed to estimate the statistical models. The EPA database is the result of the most comprehensive effort to date to compile the diesel fuels and HDD emissions test data that has been published. Notwithstanding imperfections and criticisms regarding data adequacy, the database fairly represents the state-of-knowledge in HDD emissions testing as of 2001. Nevertheless, it characterizes in numbers sufficient for statistical analysis only the engine technologies that predominate in the current on-road vehicle fleet, and provides little or no information on newer technology engines equipped with EGR, exhaust after-treatment catalysts and particulate traps, or designed with advanced engine combustion controls. 
EGR-equipped engines will soon enter the vehicle fleet and more advanced engines with exhaust aftertreatment and combustion controls are expected to be introduced in 2004 and later years in response to morestringent emission standards. As a result, the statistical models developed from the existing database must be adjusted for the effects of new technology in order to estimate fleetwide emissions impacts in future years. The following adjustment process is adopted:

- The statistical models are treated as functions that predict an emissions response benchmarked to the technology level and fuel-responsiveness of current on-road HDD engines. These functions, without further adjustment, are representative of the emissions response for current on-road engines that survive to future years.

- The fleetwide response of $\mathrm{NO}_{\mathrm{x}}$ emissions to cetane number is adjusted downward over time to account for the portion of engines equipped with EGR. $\mathrm{NO}_{\mathrm{x}}$ emissions from EGRequipped engines are assumed to be unresponsive to cetane number based on the one study that has examined this issue. The adjustment varies by calendar year based on EPA estimates of the fleetwide proportion of EGR-equipped engines.

- Once adjusted for EGR, and in the absence of advanced engine combustion controls, the predictive functions are assumed to be representative of future engines that will be equipped with exhaust after-treatment devices. That is, the percentage response of engine-out emissions to fuel changes as predicted by the EGR-adjusted functions are assumed to pass through to an equal percentage response in exhaust emissions, although the percentage is referenced to a much lower baseline level.

It is reasonable to expect that future engine designs will have reduced emissions sensitivity to fuels as a result of advances in engine combustion controls. Modifications in combustion chamber design, sophisticated computerized control of injector operation, and similar advances are expected to modify the in-cylinder combustion process to reduce emissions. These advances may exploit pathways to combustion change that are similar to the ones by which fuel reformulation reduces emissions in current engines, and thereby reduce the sensitivity of emissions to fuel reformulation. Because there is no test information on which to base an analysis of this effect, a scenario approach has been taken in which bounding assumptions are made for the sensitivity to fuels in future, advanced engines. For the portion of the fleet having advanced combustion controls, the fuel sensitivity is assumed to range from one-third less than current engines to the limit of zero response to fuels.

\section{II-1.4 ORGANIZATION OF THE REPORT}

Section 2 of this report presents a summary of the analytical methodology and the database used in the study. The discussion emphasizes the incorporation of nonlinear (quadratic and interactive) terms in the variable space used in the eigenfuel analysis. Additional, more detailed information on the eigenfuel methodology can be found in previously referenced ORNL publications, while further information on the database can be found in the previously referenced EPA and SwRI publications. Section 3, and the related Appendices A and $\mathrm{B}$, present a summary of the statistical results for the eigenfuel emission models. The emphasis in this section is the overall composition of the vector models and the contributions made by the nonlinear terms.

To accommodate implementation within RYM, the eigenvector models are transformed to a mathematicallyequivalent form in terms of fuel property variables. Section 4 describes this process and presents the resulting RYM emission response models. The coefficients of the model terms are presented and the predicted effects of fuel properties on emissions are displayed. Section 5 then describes the process by which the models are adjusted for technology effects and implemented with RYM. 



\section{II-2. METHODOLOGY AND DATA}

\section{II-2.1 DIESEL FUEL AND EMISSIONS DATABASE}

One result of the 2001 work by EPA on diesel fuel characteristics and engine emissions was the creation of a database containing more detailed information on a wider range of heavy-duty diesel engines and fuels than had been previously available. A full description of the database can be found in EPA 2001, pp. 6-22.

A subset of the EPA database, consisting of approximately 70 percent of its tests, was used in the prior ORNL work on this topic. The subset spans ten different engine technology groups, including the dominant technology types on the road ${ }^{1}$, which were found in the EPA work to share a common emissions response to fuels. The subset contains 906 emissions tests, out of the 1315 tests in the entire database, for which $\mathrm{NO}_{\mathrm{x}}$ and PM emissions and a minimum of nine different fuel properties had been measured. When reduced to eliminate test data on very high-sulfur fuels (discussed below), the data set used here contains a total of 707 tests.

The sulfur content of fuels in the EPA database follows a bi-model distribution. A majority of the fuels are below the current federal standard for on-road fuels of $500 \mathrm{ppm}$ sulfur, although a substantial number have sulfur contents in excess of $1000 \mathrm{ppm}$. EPA retained all of the data, arguing that high-sulfur fuels were still permitted in off-road applications. For this work, test data for fuels of $750 \mathrm{ppm}$ sulfur or less have been retained, so that the analysis better represents the lower sulfur levels of current and future diesel fuels.

As shown in Figure 2.1, a majority of the data are clustered in the range of 350-500 ppm sulfur. A smaller, but still substantial, number of tests have been conducted at reduced sulfur levels in the ranges of $200 \mathrm{ppm}$, $100 \mathrm{ppm}$, and below $50 \mathrm{ppm}$. Overall, the data average $322 \mathrm{ppm}$ sulfur, which is very near the $350 \mathrm{ppm}$ average for current commercial fuels, and represent reasonably well the very low sulfur levels that will be characteristic of future fuels.

The statistical models developed from the data subset can be used directly to represent the emissions response to fuels in the engines of the current on-road fleet and in that portion of future fleets that survive from today. Because there is inadequate (or no) data to support statistical analysis on engines with EGR, exhaust aftertreatment, or advanced engine combustion controls, the statistical models developed for current technologies have been adjusted in an effort to account for possible differences in the fuels-emissions response of future engines. The statistical models are treated as emissions response functions that are benchmarked to current technology levels and fuel-responsiveness and can, therefore, be re-calibrated by the adjustment process to represent future technologies.

\section{II-2.2 FUEL VARIABLE SPACE}

Past ORNL work used twelve primary physical, chemical, and compositional properties to describe diesel fuels. Twelve primary properties are still used to describe fuels in this work (see Table 2.1), but the representation of cetane number has changed. Total cetane number and cetane improvement are now used in place of natural cetane number and cetane improvement, in recognition of the belief that natural and

\footnotetext{
1 The dominant technology groups are Groups T and F, consisting of low-speed turbocharged engines below 500 horsepower with electronic fuel injection (Group T) and mid-speed turbocharged engines of any horsepower rating with mechanical fuel injection (Group F). Engines in these groups are not equipped with EGR systems, oxidations catalysts, or particulate traps.
} 
Figure 2.1. Distribution of Fuel Sulfur Content

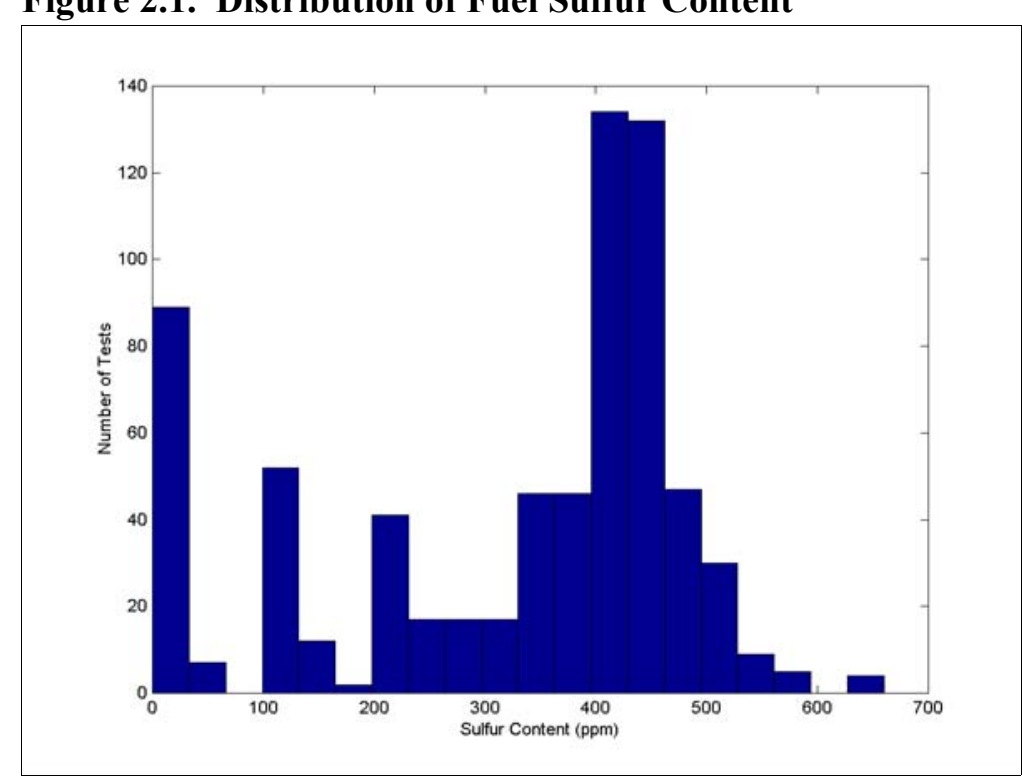

Table 2.1. Diesel Fuel Properties

\begin{tabular}{|c|c|c|}
\hline Fuel Property & Units & Description \\
\hline Total Cetane & number & total cetane number \\
\hline Cetane Difference & number & cetane number increase due to additives \\
\hline Specific Gravity & $\mathrm{gm} / \mathrm{cm}^{3}$ & \\
\hline Viscosity & $\mathrm{mm}^{2} / \mathrm{sec}$ & at/near 40 degrees $\mathrm{C}$ \\
\hline Sulfur Content & ppm & \\
\hline Aromatics Content & vol percent & \\
\hline IBP & Fahrenheit $^{\circ}$ & Initial boiling point \\
\hline $\mathrm{T} 10$ & Fahrenheit $^{\circ}$ & 10 percent evaporation temperature \\
\hline $\mathrm{T} 50$ & Fahrenheit ${ }^{\circ}$ & 50 percent evaporation temperature \\
\hline T90 & Fahrenheit $^{\circ}$ & 90 percent evaporation temperature \\
\hline FBP & Fahrenheit $^{\circ}$ & Final boiling point \\
\hline Oxygen Content & wt $\%$ & Fuel oxygen content \\
\hline
\end{tabular}

additized cetane should have equivalent emissions effects. With this variable choice, additized cetane contributes to the model in two places. We can think of its presence in the total cetane term as representing the effect it shares in common with natural cetane. Emissions effects, if any, that are attributed to the cetane improvement term alone will tell us the extent to which additized cetane differs from natural cetane. 
Prior ORNL work also examined emission models based solely on linear fuel property variables. For the first time, this work takes the step of considering nonlinear (quadratic and interactive) terms in the variable space used in the eigenfuel analysis. Quadratic $\left(\mathrm{X}^{2}\right)$ terms are used to represent saturation effects, in which the emission response slows and then ends as a fuel characteristic is varied to an ultimate value. Interactive terms are used to represent effects by which changes in two fuel properties tend to potentiate or offset each other. With twelve variables, as many as twelve quadratic (squared) terms and $12 * 11 / 2=66$ interaction terms are possible, for a total of 78 second order terms that could be considered.

For this work, quadratic and interactive terms were constructed for the six fuel properties that previous research had found to be important predictors for emissions (total cetane, cetane improvement, specific gravity, sulfur content, aromatics content, and oxygen content). Nonlinear terms were not considered for viscosity and the distillation temperatures, because these properties are thought to be most useful as descriptors of the fuels, rather than emissions. Thus, 6 quadratic and $6 * 5 / 2=15$ interaction terms were considered for a total of 21 nonlinear terms. Much of the work in model development is related to determining which of the nonlinear terms make a useful contribution.

\section{II-2.3 METHODOLOGY FOR MODEL DEVELOPMENT}

The process of model development follows the methodology laid out in prior ORNL publications (McAdams, Crawford and Hadder 2000a,b and 2002). The dependent variable is the logarithm of emissions after the effects of individual engines have been removed from the data. The variable space is defined by the choice of the twelve linear fuel property variables and one or more nonlinear terms. The linear fuel properties are used in all cases, while the number of nonlinear terms varies throughout the analysis.

Having chosen a variable space containing $\mathrm{N}$ total linear and nonlinear terms, Principal Components Analysis (PCA) is used to define the $\mathrm{N}$ eigenvectors that form an orthogonal basis for the space. In this approach, the nonlinear terms are incorporated directly in the vectors. The property-based description of fuels is transformed to eigenvector terms, and the eigenvector weights become the independent fuel variables in an otherwise conventional linear regression analysis. The effect of engines on emissions is removed in a first stage regression that essentially re-expresses the emissions test data to the mean emissions levels for each engine. The effect of fuels is assessed in a second stage regression conducted on the re-expressed emissions values. A full description of the regression methodology can be found in McAdams 2000b, pp. 9-32.

There are two basic methods to incorporate nonlinear terms in a regression model. In the "post normalization" method, variables $\mathrm{X}$ and $\mathrm{X}^{2}$ are formed and then independently normalized to mean 0 and standard deviation 1 . This method is computationally simple, but one should recognize that $\mathrm{X}$ and $\mathrm{X}^{2}$ will typically exhibit a strong linear correlation when computed over a range of positive values. In the "prenormalization" method, variable X is first normalized and squared to form $\mathrm{X}^{2}$, which is then renormalized. This method is computationally more complex, but substantially reduces the correlation between linear and nonlinear terms that would otherwise be present. The "pre-normalization" method was chosen here over the competing "post-normalization" approach because it greatly reduces the linear dependence among terms.

The resulting emission models are of the form:

$$
\log (\mathrm{E})=\mathrm{A}_{0}+\operatorname{SUM}\left(\mathrm{A}_{\mathrm{i}} * \mathrm{~W}_{\mathrm{i}}\right)
$$

where $\mathrm{A}_{\mathrm{i}}$ are linear regression coefficients and $\mathrm{W}_{\mathrm{i}}$ is the fuel weight for eigenvector $\mathrm{I}$. This model form implies that mass emissions are an exponential function of the summation term:

$$
\mathrm{E}=\mathrm{E}_{0} * \operatorname{EXP}\left(\operatorname{SUM}\left(\mathrm{A}_{\mathrm{i}} * \mathrm{~W}_{\mathrm{i}}\right)\right)
$$


As shown in the ORNL publications, any eigenvector model can be transformed into a mathematically equivalent model in terms of the original fuel property variables:

$$
\begin{aligned}
\log (\mathrm{E}) & =\operatorname{SUM}\left(\mathrm{B}_{\mathrm{i}} * \mathrm{P}_{\mathrm{i}}\right), \text { or } \\
\mathrm{E} & =\operatorname{EXP}\left(\operatorname{SUM}\left(\mathrm{B}_{\mathrm{i}} * \mathrm{P}_{\mathrm{i}}\right)\right)
\end{aligned}
$$

where $B_{i}$ are emissions coefficients and $P_{i}$ is the value for fuel property $I$.

While the models are exponential when stated in terms of mass emissions, the degree of non-linearity introduced by the transformation is small in all cases, because the summation term is, itself, relatively small. A greater degree of nonlinear behavior can be introduced by the presence of quadratic terms, although this is found to be modest in most circumstances.

The process of model development is a sequential search to find the most efficient set of variables for predicting emissions. The search is done independently in developing the $\mathrm{NO}_{\mathrm{x}}$ and $\mathrm{PM}$ emission models, so that the terms included in the variable space will differ for the two pollutants. A decision was made at the outset of the study to retain all twelve linear fuel properties. Thus, the emphasis in the search is to identify nonlinear terms that make useful contributions to prediction.

Starting with a variable space containing only the twelve linear fuel property variables, an eigenvector model is developed using the methods previously described. The 21 quadratic and interactive terms are tested individually against the residuals from the best eigenvector model to identify terms that may add predictive power. Quadratic and interactive terms appearing to contribute to predictive power are then added to the linear terms to create an augmented variable space.

The process then follows the following steps until all of the nonlinear terms that make useful contributions are identified:

1. A new set of eigenvectors is defined for the augmented variable space, which now includes both linear and nonlinear variables, and a new eigenvector model is selected, considering the significance and substantiality of terms. Typically, the retained terms account for 92-94 percent of the total effect associated with fuels.

2. The non-linear fuel property term making the weakest contribution to the model is identified using the simplify.m algorithm that has been developed for use in variable selection (see McAdams 2002, Appendix D). The algorithm provides a means to attribute the eigenvector model sums of squares (SS) to the individual fuel properties and to conduct an F test of their significance.

3. When a non-linear term is dropped from consideration, the eigenvectors are redefined and a revised eigenvector model is selected. The nonlinear terms not present in the model are then re-tested individually against the residuals from the current model to identify terms that may add predictive power. One such term can be introduced to the model at each step. We then return to step 1 above and repeat the model estimation, simplification and testing process.

When the model development process came to an end, the final eigenvector models for $\mathrm{NO}_{\mathrm{x}}$ and $\mathrm{PM}$ were based on variable spaces of 17 and 19 terms, respectively. As shown in Table 2.2, the variable spaces contain all twelve linear terms plus five and seven nonlinear terms. 
Table 2.2. Terms Contained in Emission Models

\begin{tabular}{|c|c|}
\hline NO ${ }_{x}$ Model & PM Model \\
\hline 12 linear fuel properties & 12 linear fuel properties \\
\hline Total Cetane ${ }^{2}$ & Total Cetane ${ }^{2}$ \\
\hline Sulfur ${ }^{2}$ & Sulfur ${ }^{2}$ \\
\hline Aromatics $^{2}$ & Oxygen $^{2}$ \\
\hline Cetane Improvement x Specific Gravity & Cetane Improvement $\mathrm{x}$ Total Cetane \\
\hline \multirow[t]{3}{*}{ Cetane Improvement x Aromatics } & Sulfur x Cetane Improvement \\
\hline & Sulfur x Specific Gravity \\
\hline & Sulfur x Aromatics \\
\hline
\end{tabular}





\section{II-3. EIGENVECTOR EMISSIONS MODELS}

This section briefly presents the eigenvector based emissions models developed to support this work. The presentation is for the reader having a familiarity with the eigenfuel approach. Supporting statistical results for the $\mathrm{NO}_{\mathrm{x}}$ and $\mathrm{PM}$ emissions models are presented in Appendices A and $\mathrm{B}$, respectively. The appendix tables give the eigenvectors of the variable spaces used in the models and the results of the two-stage regression analysis. The following discussion highlights important characteristics of the models emphasizing the nonlinear terms that have been incorporated in the models.

\section{II-3.1 NO $\mathrm{NO}_{\mathrm{x}}$ EMISSIONS MODEL}

The eigenvector model for $\mathrm{NO}_{\mathrm{x}}$ emissions consists of 6 vector terms that account for nearly 92 percent of the total effect related to fuels, as measured by the model SS attributed to fuels. Four eigenvectors in the model (numbers 1,2, 4, and 9) make large individual contributions to the fuels-related effect. Two other vectors (numbers 5 and 6) make smaller, but still significant, contributions and were retained in the model. The partitioning of the fuels-related effect for $\mathrm{NO}_{\mathrm{x}}$ among the eigenvectors is shown in Figure 3.1.

Figure 3.1. Sum of Squares Contributions to $\mathrm{NO}_{\mathrm{x}}$ Model

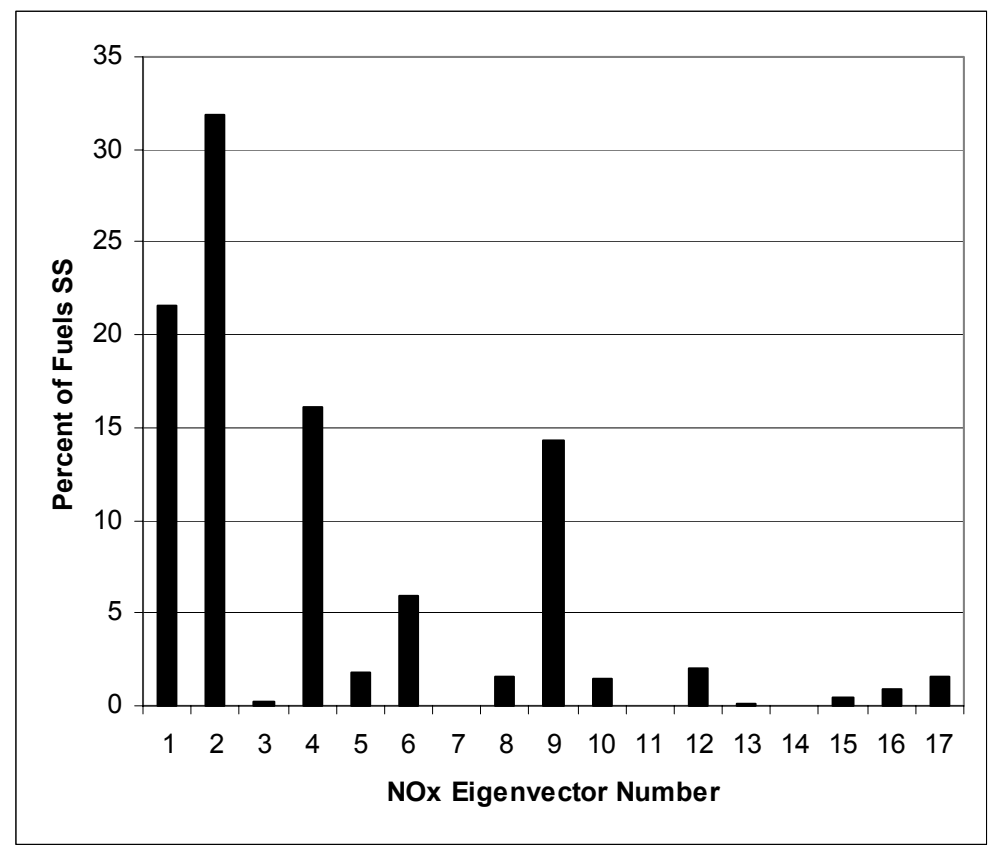

Though derived from an expanded variable space containing both linear and non-linear fuel property variables, the eigenvectors influencing $\mathrm{NO}_{\mathrm{x}}$ emissions are similar in many respects to the vectors seen in other fuels data sets:

- Vector 1 ( $22 \%$ of fuels SS) is related to the heaviness of the fuel.

- Vector 2 (32\% of fuels SS) represents a cetane "constellation" involving total cetane, cetane improvement, and interactive terms of cetane improvement with specific gravity and aromatics content. 
- Vector 4 (16\% of fuels SS) is similar to the vector previously identified as representing light cycle oil, in that it loads heavily on aromatics content and density.

- Vector 6 (9\% of fuels SS) is related to cetane improvement.

Cetane improvement by itself should be interpreted as representing its effect, if any, over and above its contributions to the emissions response for total cetane. The relatively smaller contributions of Vector 6 , and the subsequent findings that the cetane variable makes only small contributions to the SS indicate that natural and additized cetane are largely equivalent in terms of their $\mathrm{NO}_{\mathrm{x}}$ effects, although differences in detail may be present.

Table 3.1 shows the contributions of the individual variables to the fuels-related SS. The most important variables are: total cetane and its quadratic term, specific gravity, aromatics content and its quadratic term, and IBP. Combined, these terms account for 89 percent of the fuels-related effect on $\mathrm{NO}_{\mathrm{x}}$. Two of the linear variables (T50 and FBP) are judged not to make significant contributions to emissions, but are retained as part of the basis of linear fuel property variables used to describe the fuels. All of the nonlinear terms make significant contributions based on application of the F-test. The nonlinear terms not present in the model were tested against the residuals, but none were found to make a significant contribution to predictive power.

Table 3.1. Sum of Squares Contributions by Fuel Property for the NO $_{x}$ Emissions Model

\begin{tabular}{lrc}
\hline \multicolumn{1}{c}{ Variable } & \multicolumn{2}{c}{$\begin{array}{c}\text { Contribution to } \\
\text { fuels-related SS }\end{array}$} \\
\hline Total Cetane & 22.7 & $*$ \\
Total Cetane $^{2}$ & 0.5 & $*$ \\
Cetane Improvement $^{\prime}$ & 1.0 & $*$ \\
CImp x SpGrav & 0.7 & $*$ \\
CImp x Aromatics & 2.4 & $*$ \\
Specific Gravity & 20.0 & $*$ \\
Viscosity & 0.5 & $*$ \\
Sulfur & 2.9 & $*$ \\
Sulfur & \\
Aromatics & 1.0 & $*$ \\
Aromatics & \\
IBP & 35.8 & $*$ \\
T10 & 3.3 & $*$ \\
T50 & 6.5 & $*$ \\
T90 & 0.6 & $*$ \\
FBP & 0.2 & \\
Oxygen & 0.4 & $*$ \\
\hline
\end{tabular}

* Indicates SS contribution is significant at the $\mathrm{p}=0.05$

level based on the F-test. 


\section{II-3.2 PM EMISSIONS MODEL}

The eigenvector model for PM emissions consists of 7 vector terms that account for 95 percent of the total effect of fuels as measured by the sum of squares. Five of the eigenvectors in the model $(1,3,5,7$, and 9) make large individual contributions to the fuels-related effect. Two other vectors (11 and 12) make smaller, but still significant, contributions and were retained in the model. The partitioning of the fuels-related effect for PM is shown in Figure 3.2.

\section{Figure 3.2. Sum of Squares Contributions to PM Emissions}

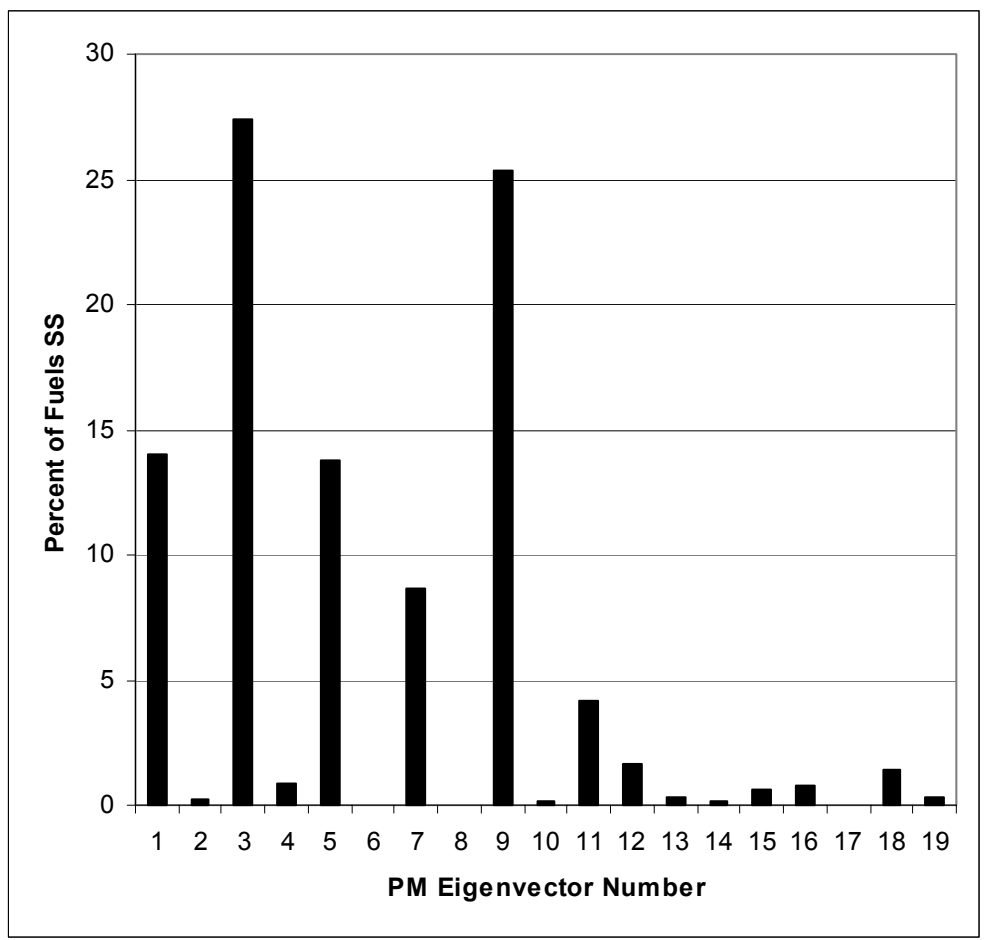

Because the variable space used for the PM model differs from that used in the $\mathrm{NO}_{\mathrm{x}}$ model in terms of the nonlinear variables included, the composition and interpretation of the eigenvectors will also differ. In spite of this difference, and the inclusion of nonlinear terms, the eigenvectors influencing PM emissions are similar in many respects to the features seen in other fuels data sets:

- Vector $1(14 \%$ of fuel SS) is related to the heaviness of the fuel.

- Vector 3 (27\% of fuel SS) is related to oxygen content.

- Vector 5 (14\% of fuel SS) is similar to the vector previously identified as representing light cycle oil.

- Vector 7 ( $9 \%$ of fuel SS) is a feature loading on IBP in conjunction with aromatics and sulfur content.

- Vector 9 (25\% of fuel SS) is related to cetane improvement.

These five vector terms account for 89 percent of the fuels-related effect. The two remaining terms in the model appear to describe interactions among fuel properties. 
Table 3.2 shows the contributions of the individual variables to the fuels-related SS for PM. The most important variables are: total cetane and its related quadratic and interactive terms, aromatics content, and oxygen content and its quadratic term. Combined, these terms account for more than 70 percent of the fuelsrelated effect on PM.

Table 3.2. Sum of Squares Contributions by Fuel Property for the PM Emissions Model

\begin{tabular}{lrc}
\hline \multicolumn{1}{c}{ Variable } & \multicolumn{2}{c}{$\begin{array}{c}\text { Contribution to } \\
\text { fuels-related SS }\end{array}$} \\
\hline Total Cetane & 11.3 & $*$ \\
Total Cetane ${ }^{2}$ & 8.7 & $*$ \\
TCet x CImp & 7.9 & $*$ \\
Cetane Improvement & 0.6 & \\
Specific Gravity & 9.4 & $*$ \\
Viscosity & 0.2 & \\
Sulfur & 2.7 & $*$ \\
Sulfur & \\
Sulfur x CImp & 3.8 & $*$ \\
Sulfur x SpGrav & 2.2 & $*$ \\
Sulfur x Aromatics & 0.8 & $*$ \\
Aromatics & 1.4 & $*$ \\
IBP & 30.6 & $*$ \\
T10 & 1.9 & $*$ \\
T50 & 0.9 & $*$ \\
T90 & 0.1 & \\
FBP & 2.3 & $*$ \\
Oxygen & 1.3 & $*$ \\
Oxygen &
\end{tabular}

* Indicates SS contribution is significant at the $\mathrm{p}=0.05$ level based on the F-test.

Three of the linear variables (cetane improvement, viscosity, T50) are judged not to make significant contributions to emissions, but are retained as part of the linear basis used to describe the fuels. That cetane improvement makes insignificant contributions to explaining PM emissions supports the position that natural and additized cetane are largely equivalent in their emissions effects. All of the nonlinear terms make significant contributions based on application of the F-test. The nonlinear terms not present in the model were tested against the residuals, but none were found to make a significant contribution to predictive power. 


\section{II-4. EMISSIONS RESPONSE MODELS}

\section{II-4.1 INTRODUCTION}

As has been noted, an eigenvector model can be transformed into a mathematically equivalent expression in terms of the original fuel property variables. An equivalent expression of this kind will give the same emissions predictions as the eigenvector model and may be easier to implement for predictive purposes in many applications. RYM is such an application, and the emissions models are therefore implemented as their equivalent fuel property expressions.

An eigenvector model can be translated to its fuel property equivalent by an analytical process of algebraic expansion and manipulation. The eigenvector weights in the regression equation would be replaced by their algebraic calculation in terms of fuel property values, and the resulting terms then re-organized into a similar equation in terms of fuel property variables. The process is cumbersome, however, and would be complicated by the two levels of variable standardization involved in the pre-normalization approach to modeling nonlinear terms.

A regression technique has been used as a curve fitting tool as an alternative to the analytical process for transforming an eigenfuel model to its equivalent in fuel property terms. The curve fitting process generates synthetic emission tests data and fits a regression model containing linear and quadratic terms in the original fuel variables, as appropriate. The result of this process is a reduced form version of the original eigenvector model that, for all practical purposes, gives equivalent predictions of emissions changes.

To develop the reduced form models, $\mathrm{N}=10,000$ synthetic diesel fuels were generated by sampling randomly from the range of fuel properties that might be encountered in refinery analyses. The range (see Table 4.1) was selected to bracket the maximum change in finished fuel properties likely to be relevant to emissions, based on the following considerations:

- The end of the range most favorable to emissions reduction was taken from the largest property changes, in the direction of reduced emissions, found in experimental fuels used in HDD emissions testing. This choice assumes that past research has identified the maximum extent that each property is likely to be modified in an effort to reduce emissions.

- The end of the range most adverse to emissions was taken from the end of the range of property values, in the direction opposite to reduced emissions, found in commercial fuels. This choice assumes that RYM need not consider finished fuels that are more adverse to emissions than those at the end of the range of current commercial fuels.

In both cases, the endpoints of the range of data (experimental or commercial) were taken as the limits of the interval that contained 95 percent of the fuels.

Having generated the fuels, the eigenfuel-based emission models were evaluated to generate a predicted emissions response. The predicted responses were then referenced to the emissions level of the average commercial fuel by expressing the predicted response as a fractional difference from the emissions value predicted for the average commercial fuel. A fractional difference of -0.050 for a fuel would mean that it reduces emissions by 5 percent compared to the average commercial fuel.

The reduced form models are estimated by regressing the synthesized fractional emissions response values against the linear, quadratic, and interaction terms found in the variable space of the eigenfuel-based models. The regression approach fits a fuel property equation to the emissions response surface of the eigenfuel 
Table 4.1. Relevant Range of Fuel Property Values

\begin{tabular}{llccc}
\hline & & $\begin{array}{c}\text { Low } \\
\text { Emissions }\end{array}$ & $\begin{array}{c}\text { Average } \\
\text { Commercial } \\
\text { Fuel }\end{array}$ & $\begin{array}{c}\text { High } \\
\text { Emissions }\end{array}$ \\
\hline TotCetane & number & 60.0 & 45.4 & 40.0 \\
CetImprv & number & 15.0 & 0 & 0.0 \\
Density & ${\mathrm{gm} / \mathrm{cm}^{3}}^{2}$ & 0.814 & 0.850 & 0.864 \\
Viscosity & $\mathrm{mm}^{2} / \mathrm{sec}$ & 1.66 & 2.67 & 3.26 \\
Sulfur & $\mathrm{ppm}$ & 5 & 352 & 500 \\
Aromatics & vol percent & 5.0 & 33.2 & 42.0 \\
IBP & deg F & 175 & 349 & 400 \\
T10 & deg F & 354 & 429 & 463 \\
T50 & deg F & 427 & 513 & 542 \\
T90 & deg F & 522 & 607 & 631 \\
FBP & deg F & 590 & 653 & 679 \\
Oxygen & wt percent & 5 & 0 & 0 \\
\hline
\end{tabular}

model, and it also reduces the response surface from one stated in terms of $\log (\mathrm{emissions})$ to one in terms of mass emissions. The functions are of the form:

$$
E=A_{o}+\operatorname{Sum}\left(A_{i}^{*} P_{i}\right)
$$

where E represents the predicted mass emissions (gm/bhp-hr), $\mathrm{A}_{\mathrm{i}}$ are the reduced-form regression coefficients, and $P_{i}$ represents the series of fuel property values including linear, quadratic, and interactive terms in the physical units shown in the table above.

The reduced-form models created by this process are excellent representations of the eigenfuel-based emissions models. The $\mathrm{R}^{2}$ statistics are 0.999 for $\mathrm{NO}_{\mathrm{x}}$ and 0.994 for $\mathrm{PM}$, and the correlation coefficients between the synthetic data and the predictions of the reduced-form models are similarly high. The use of $\mathrm{N}=10,000$ synthetic fuels assures that the estimated coefficient values are influenced by sampling fluctuations to only a very small degree. The smallest $t$-statistic in the $\mathrm{NO}_{\mathrm{x}}$ model was 67 for the FBP term. The smallest $\mathrm{t}$-statistic in the PM model was 14 for the T10 term.

The following sections present the $\mathrm{NO}_{\mathrm{x}}$ and $\mathrm{PM}$ response models, in turn, including the coefficient values and the magnitudes of predicted emissions effects. Although we show predictions for the fuel properties individually, it must be remembered that the properties generally do not vary independently in real-world fuels. The predictions seen here for individual properties should be understood as vector emission effects that have been distributed to the individual properties. In the case of a typical fuel change, several (or many) properties will change simultaneously, and the resulting emissions response will be the sum of contributions that are attributed to the properties.

\section{II-4.2 NO $\mathrm{NO}_{\mathrm{x}}$ RESPONSE MODEL}

Table 4.2 presents the reduced-form $\mathrm{NO}_{\mathrm{x}}$ response model. The equations predict the fractional emissions change relative to the average commercial fuel as a function of the fuel property values (in physical units). The equations for total cetane, sulfur content, and aromatics content include both linear and quadratic contributions. Cetane improvement appears in five places in the equation, including its participation in total 
Table 4.2. Reduced-Form NO $\mathrm{N}_{\mathrm{x}}$ Response Model (coefficients in physical units)

\begin{tabular}{lccc}
\hline \multicolumn{1}{c}{ Model Term } & $\begin{array}{c}\text { Linear } \\
\text { Coefficient }\end{array}$ & $\begin{array}{c}\text { Quadratic } \\
\text { Coefficient }\end{array}$ & $\begin{array}{c}\text { Inflection } \\
\text { Point }\end{array}$ \\
\hline Intercept & -0.29436 & & \\
Total Cetane & -0.0050089 & 0.00003002 & 83.4 \\
Cetane Improvement & -0.044716 & & \\
$\quad$ CImp x Sp Gravity & 0.054009 & & \\
$\quad$ CImp x Aromatics & -0.00005762 & & \\
Specific Gravity & 0.37573 & & \\
Viscosity & -0.0023846 & & \\
Sulfur & 0.00007831 & -0.08262 E-6 & 473.9 \\
Aromatics & 0.0043869 & -0.00004791 & 45.8 \\
IBP & 0.00014618 & & \\
T10 & -0.00002595 & & \\
T50 & -0.00005585 & & \\
T90 & 0.00002215 & & \\
FBP & 0.00001847 & & \\
Oxygen & 0.0093568 & & \\
\hline
\end{tabular}

cetane (linear and quadratic terms), its own linear term, and two interactive terms with specific gravity and aromatics.

The three properties with quadratic terms will reach a maximum when the property value equals the inflection points shown in the table. Past practice in emissions modeling, as in the Complex Model for Reformulated Gasoline (DOE 1994), has been to "straight-line" the emissions response for values beyond the inflection point. That approach is adopted here, although as a practical matter, all current and future fuels will lie below the inflection points, so that the straight-line portion of the curves will not be encountered.

Figures 4.1 through 4.5 illustrate the predicted $\mathrm{NO}_{\mathrm{x}}$ responses to the fuel properties found to have the largest effects:

- NOx emissions are predicted to decrease by 2.7 percent when total cetane number is raised 15 points from the commercial average of 45.4 to a total of 60 cetane (Figure 4.1), assuming that other fuel property values would remain constant. There is a modest curvature to the relationship. A slowing of the emissions response begins to appear at total cetane values in the mid-50s, and the response continues to slow as higher cetane numbers are approached. The maximum predicted reduction is 4.3 percent at the inflection point of 83.4 total cetane. This high cetane level is very unlikely to be encountered in either analytical exercises or in real-world practice. 
Figure 4.1. $\mathrm{NO}_{\mathrm{x}}$ Emissions Response to Total Cetane Number

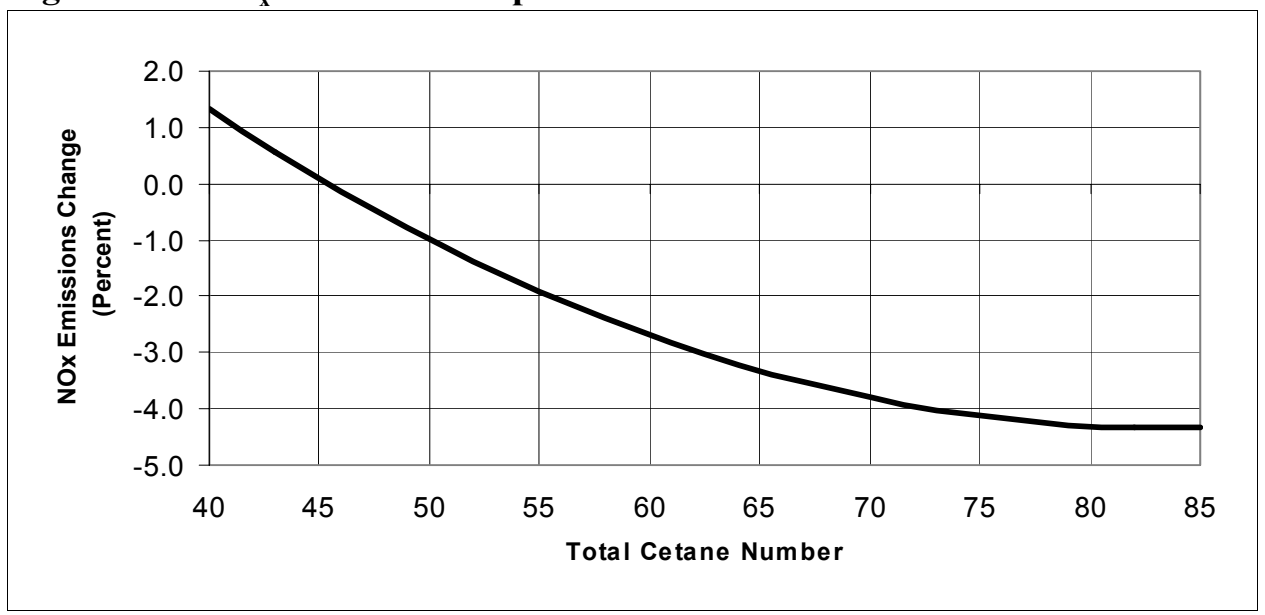

Figure 4.2. $\mathrm{NO}_{\mathrm{x}}$ Emissions Response to Natural and Additized Cetane

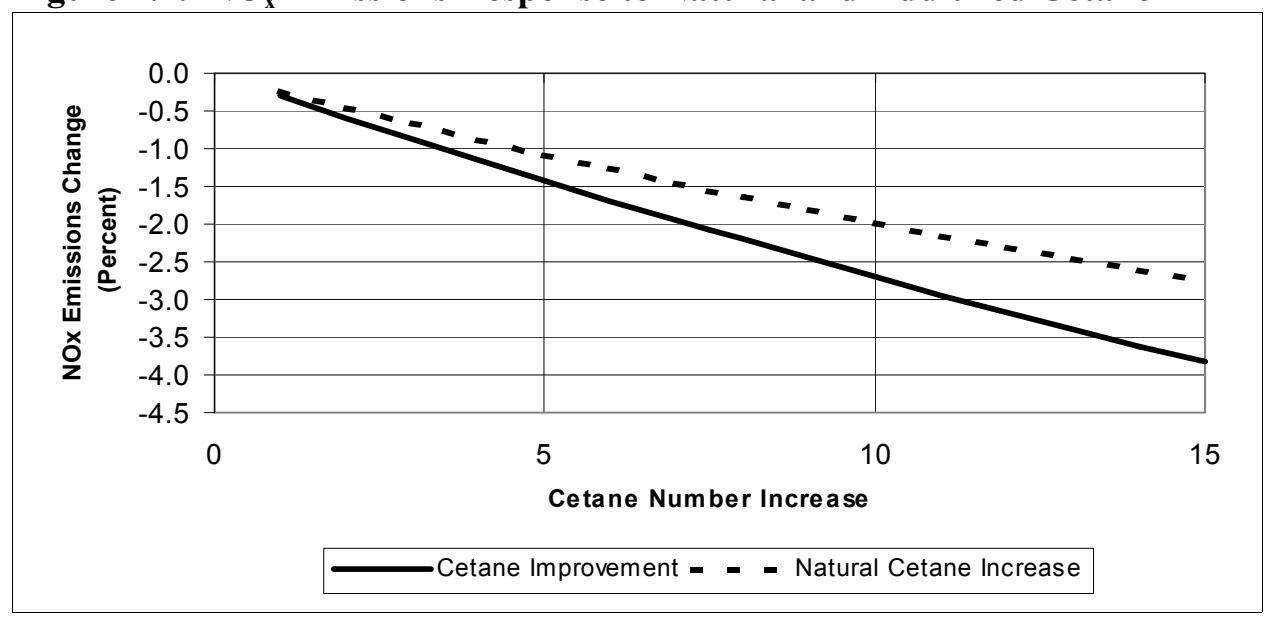

Figure 4.3. $\mathrm{NO}_{\mathrm{x}}$ Emissions to Atomatics Content

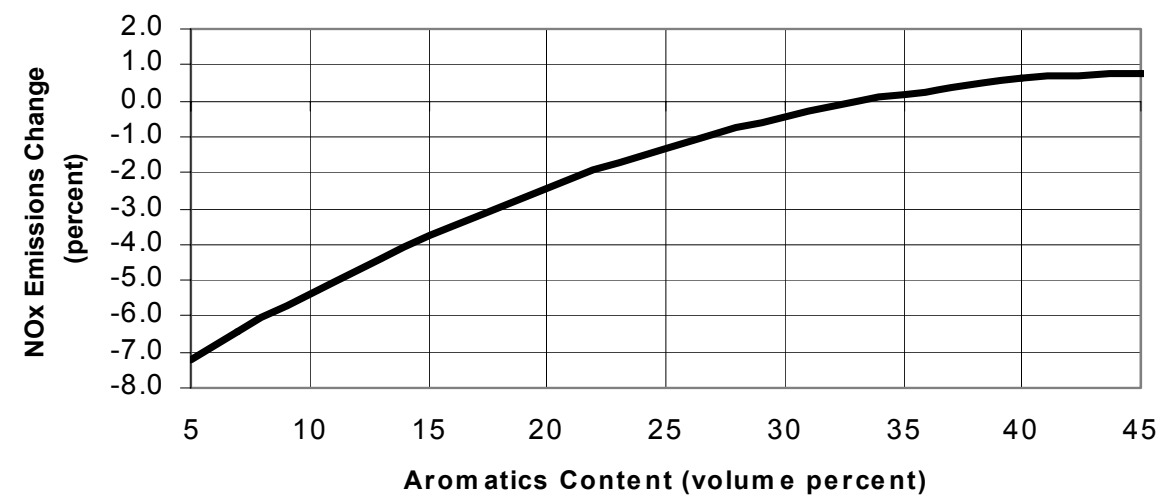


Figure 4.4. NO $_{x}$ Emissions Response to Sulfur Content

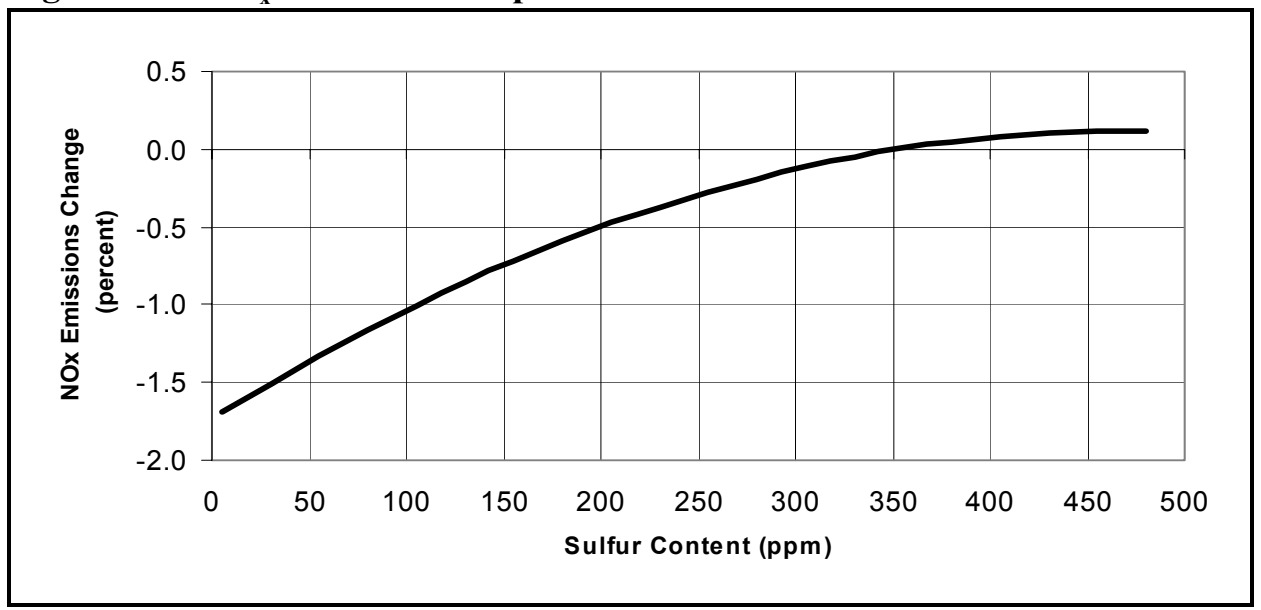

Figure 4.5. NO Emissions Response to Oxygen Content

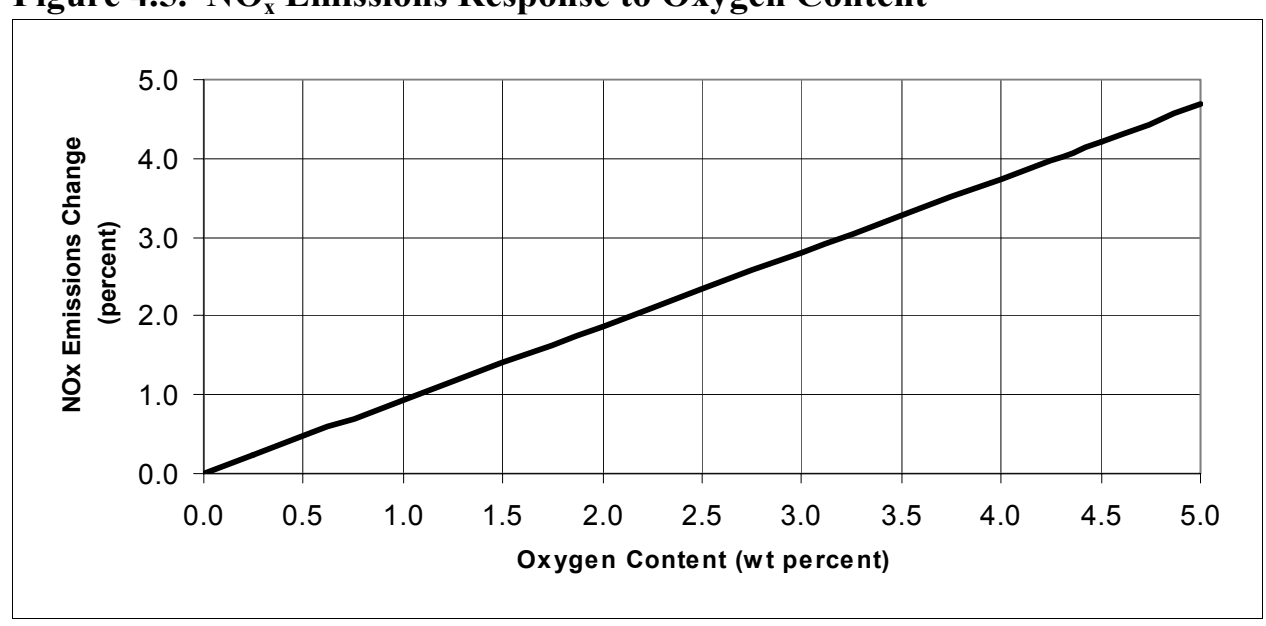

- NOx emissions decrease by 7 percent when the aromatics content is reduced to 5 percent, from its commercial average of 33.2 percent (Figure 4.3). The saturation effect occurs at the high end of the range for aromatics contents above 45.8 volume percent. Moving from the current commercial average to lower aromatics content, a very modestly accelerating rate of emissions reduction is seen.

- Reducing sulfur content has little initial effect on NOx emissions when starting near the inflection point of $474 \mathrm{ppm}$ (Figure 4.4), but a modestly accelerating emissions reduction begins as the sulfur content is reduced below $400 \mathrm{ppm}$. Overall, NOx emissions are predicted to decline by 1.7 percent compared to current commercial fuels as the sulfur content is reduced to levels of $15 \mathrm{ppm}$ or less.

- Oxygen content is predicted to have an adverse effect on NOx emissions (Figure 4.5). Adding 5 percent oxygen (by weight) to a diesel fuel is predicted to increase $\mathrm{NO}_{\mathrm{x}}$ emissions by nearly 5 percent. The magnitude of this effect is very similar to what has been seen with biodiesel fuels (Grabowski 2000). 
It is important to understand these predicted effects as attributed emissions responses. In the case of sulfur content, for example, we do not interpret the response as meaning that sulfur has a direct effect in reducing $\mathrm{NO}_{\mathrm{x}}$ formation. This is a specific example of the general caveat stated earlier: the predictions seen here are vector emission effects that have been distributed to the individual properties as a calculation convenience for implementation in the RYM model. In typical applications, several (or many) properties will change simultaneously, and the resulting emissions response will be the sum of contributions that are attributed to the properties.

The emissions models were formulated with total cetane and cetane improvement as separate variables based on the expectation that natural and additized cetane are nearly equivalent. The primary cetane effect is carried by the total cetane terms (linear and quadratic), while the cetane improvement term and its interactions act as relatively small perturbations to the basic cetane effect. The structure of the emissions model implies that the $\mathrm{NO}_{\mathrm{x}}$ emission change will saturate as higher total cetane levels are approached through any combination of natural and additized cetane.

The interactive effects of cetane improvement with specific gravity and aromatics content have not previously been seen in diesel emissions analysis. These interactions pertain to the modifying the effect of cetane improvement beyond that expected from its contribution to total cetane. The interaction of cetane improvement with aromatics indicates that, for a fixed increment in cetane number, more emissions benefit occurs in high aromatics fuels (typically low natural cetane) and less emissions benefit in low aromatics fuels (typically high natural cetane). Conversely, for a fixed increment in cetane number, less emissions benefit is predicted to occur in fuels of high specific gravity, while more benefit is predicted for fuels of low specific gravity.

The interaction terms are generally small except when evaluated for the largest changes in cetane improvement and the interactive variables. For example:

- A 10 number cetane improvement reduces the $\mathrm{NO}_{\mathrm{x}}$ emissions of the average commercial fuel (33 percent aromatics) by 2.7 percent, considering all of the cetane effects. A 20 percent aromatics fuel with 10 cetane number improvement is predicted to reduce $\mathrm{NO}_{\mathrm{x}}$ by 4.4 percent, including the effect attributed to aromatics reduction. Of the 1.7 percent incremental effect, the interaction of cetane improvement and aromatics content contributes 0.8 percent.

- For the interaction with specific gravity, a 10 cetane number improvement to a fuel with above-average density $\left(0.860 \mathrm{gm} / \mathrm{cm}^{3}\right)$ reduces $\mathrm{NO}_{\mathrm{x}}$ emissions by only 1.8 percent, compared to the 2.7 percent reduction from 10 cetane number improvement in the average commercial fuel $\left(0.850 \mathrm{gm} / \mathrm{cm}^{3}\right)$. Of the difference between the cases $(+0.9$ percent $)$, the higher specific gravity of the base fuel has the effect of increasing emissions by +0.4 percent, while the interaction term of specific gravity with cetane improvement adds an additional +0.5 percent.

Table 4.3 summarizes the magnitude of the predicted $\mathrm{NO}_{\mathrm{x}}$ emissions response over the range of fuel properties values that might be encountered in modifying fuels. The central column gives the property value found in the average commercial fuel. When emissions are decreased by raising the property value, the columns to the right of center show the predicted emissions change at the upper end of the property range. When emissions are decreased by reducing the property value, the columns to the left of center show the lower end of the range and its associated emissions change. Not surprisingly, aromatics content, total cetane, cetane improvement, and oxygen content have the largest potential impacts. Specific gravity, sulfur content and the initial boiling point have smaller effects, while viscosity and the remaining distillation temperatures have almost no effect. 
Table 4.3. Magnitude of Predicted $\mathrm{NO}_{\mathrm{x}}$ Emissions Response

\begin{tabular}{lcccccc}
\hline & & $\begin{array}{c}\text { Property } \\
\text { Value }\end{array}$ & $\begin{array}{c}\text { Emissions } \\
\text { Change } \\
\text { (percent) }\end{array}$ & $\begin{array}{c}\text { Average } \\
\text { Commercial } \\
\text { Fuel }\end{array}$ & $\begin{array}{c}\text { Property } \\
\text { Value }\end{array}$ & $\begin{array}{c}\text { Emissions } \\
\text { Change } \\
\text { (percent) }\end{array}$ \\
\hline Total Cetane & number & & & 45.4 & 60 & -2.7 \\
Cetane Impr & number & & & 0 & 15 & -1.1 \\
Specific Gravity & $\mathrm{gm} / \mathrm{cm}^{3}$ & 0.815 & -1.4 & 0.850 & & \\
Viscosity & $\mathrm{mm}^{3} / \mathrm{sec}$ & & & 2.67 & 3.25 & -0.2 \\
Sulfur & $\mathrm{ppm}$ & 5 & -1.7 & 352 & & \\
Aromatics & vol & 5 & -7.2 & 33.2 & & \\
IBP & percent & & -2.6 & 349 & & -0.1 \\
T10 & deg F & 175 & & 429 & 465 & -0.2 \\
T50 & deg F & & & 513 & 540 & \\
T90 & deg F & & -0.2 & 607 & & \\
FBP & deg F & 522 & -0.1 & 653 & & \\
Oxygen & deg F & 590 & - & 0 & 5 & \\
\hline
\end{tabular}

As a final point of reference, the $\mathrm{NO}_{\mathrm{x}}$ emissions model predicts a 4.1 percent reduction in $\mathrm{NO}_{\mathrm{x}}$ emissions for the average diesel fuel found in the Los Angeles Basin ${ }^{2}$. This fuel is one in which aromatics content has been reduced to 22 percent and the total cetane number raised to 52 . The predicted emission change is somewhat smaller than the 5.0 percent reduction predicted by earlier eigenvector models, which did not include quadratic or interactive terms that may limit the predicted response as fuel properties are modified substantially from the commercial average.

\section{II-4.3 PM RESPONSE MODEL}

Table 4.4 summarizes the coefficients of the reduced-form RYM PM emissions model. The effect of total cetane is represented by linear and quadratic terms and an interaction with cetane improvement. The incremental effect of cetane improvement (i.e., beyond its participation in total cetane) is represented by a simple linear term. Sulfur content, widely recognized as an important predictor for PM emissions, is represented by a complex equation involving linear and quadratic terms and interactions with cetane improvement, specific gravity, and aromatics. Oxygen content, also recognized as an important predictor, is represented by linear and quadratic terms.

When interaction terms are present, the inflection point for a variable becomes a function of the interacting variables, rather than a simple number. Therefore, the table also gives the formulas for computing inflection points for total cetane and sulfur content, beyond which the values of the variables and the predicted emissions response should be held constant. The oxygen terms predict a modestly accelerating emissions response to increasing oxygen content. Therefore, the mathematical inflection point occurs at negative values for oxygen content and is of no concern in application of the model.

2 See EPA 2001, Section III.F. 
Table 4.4. Reduced-Form PM Response Model

(coefficients in physical units)

\begin{tabular}{lccc}
\hline \multicolumn{1}{c}{ Model Term } & $\begin{array}{c}\text { Linear } \\
\text { Coefficient }\end{array}$ & $\begin{array}{c}\text { Quadratic } \\
\text { Coefficient }\end{array}$ & $\begin{array}{c}\text { Inflection } \\
\text { Point }\end{array}$ \\
\hline Intercept & -0.20469 & & \\
Total Cetane & -0.046802 & 0.00041817 & Eq. 1 \\
$\quad 0.00051441$ & & \\
TCet x CImp & -0.031423 & & \\
Cetane Improvement & 0.78127 & \\
Specific Gravity & -0.0057629 & & \\
Viscosity & 0.0012166 & -0.00000047 \\
Sulfur & 0.00001302 & & \\
$\quad$ Sulfur x Cimp & -0.00096997 & & \\
Sulfur x Sp Grav & -0.00000446 & & \\
Sulfur x Aromatics & 0.0046396 & \\
Aromatics & 0.00009756 & \\
IBP & -0.00003778 & \\
T10 & -0.00010789 & \\
T50 & 0.00023096 & \\
T90 & 0.00019213 & & \\
FBP & -0.024834 & & \\
\hline
\end{tabular}

Equ. 1. Compute inflection point in cetane space as follows, where notation for coefficients is defined by the equation $\mathrm{E}=\mathrm{a}$ TCet $+\mathrm{b}$ TCet $^{2}+\mathrm{c}$ CImp $+\mathrm{d}^{\text {TCet }}{ }^{*} \mathrm{CImp}+\mathrm{e} \mathrm{Sulf*CImp}$.

(a) Resolve total cetane into its components TCet $=$ NCet + CImp.

(b) Limit CImp to not greater than: $-\left[a+c+(2 b+d) N C e t+e^{*}\right.$ Sulf $] /(2 b+2 d)$.

(C) Limit NCet to not greater than: $-[a+(2 b+d) C I m p] /(2 b)$.

(d) Recompute TCet $=$ NCet + CImp after limitations are applied.

Equ 2. Compute inflection point for sulfur as follows, where notation for coefficients is defined by the equation $\mathrm{E}=\mathrm{a} \mathrm{S}+\mathrm{b} \mathrm{S}^{2}+\mathrm{c} \mathrm{S}^{*} \mathrm{SpGrav}+\mathrm{d} \mathrm{S}^{*}$ Arom $+\mathrm{e} \mathrm{S}^{*} \mathrm{CImp}$. Limit sulfur to not greater than: $-[\mathrm{a}+\mathrm{c}$ SpGrav $+\mathrm{d}$ Arom $+\mathrm{e}$ CImp $] /(2 \mathrm{~b})$

The cetane terms in the model - total cetane, its quadratic, cetane improvement and its interaction with total cetane - produce the emissions surface shown in Figure 4.6. The axes in the figure are natural cetane and cetane improvement; therefore, total cetane number increases as one moves into the figure from the foreground point at natural cetane $=40$ and cetane improvement $=0$. The $P M$ emissions surface declines rapidly from the foreground point $(40,0)$, whether moving along the axis of increasing natural cetane, the axis of 
increasing cetane improvement, or diagonally through the figure. One sees that the emissions response quickly saturates as higher total cetane numbers are approached. The maximum PM emissions reduction is -5.0 percent at a total cetane number of 56 or higher.

Figure 4.6. PM Emissions Response to Cetane Effects

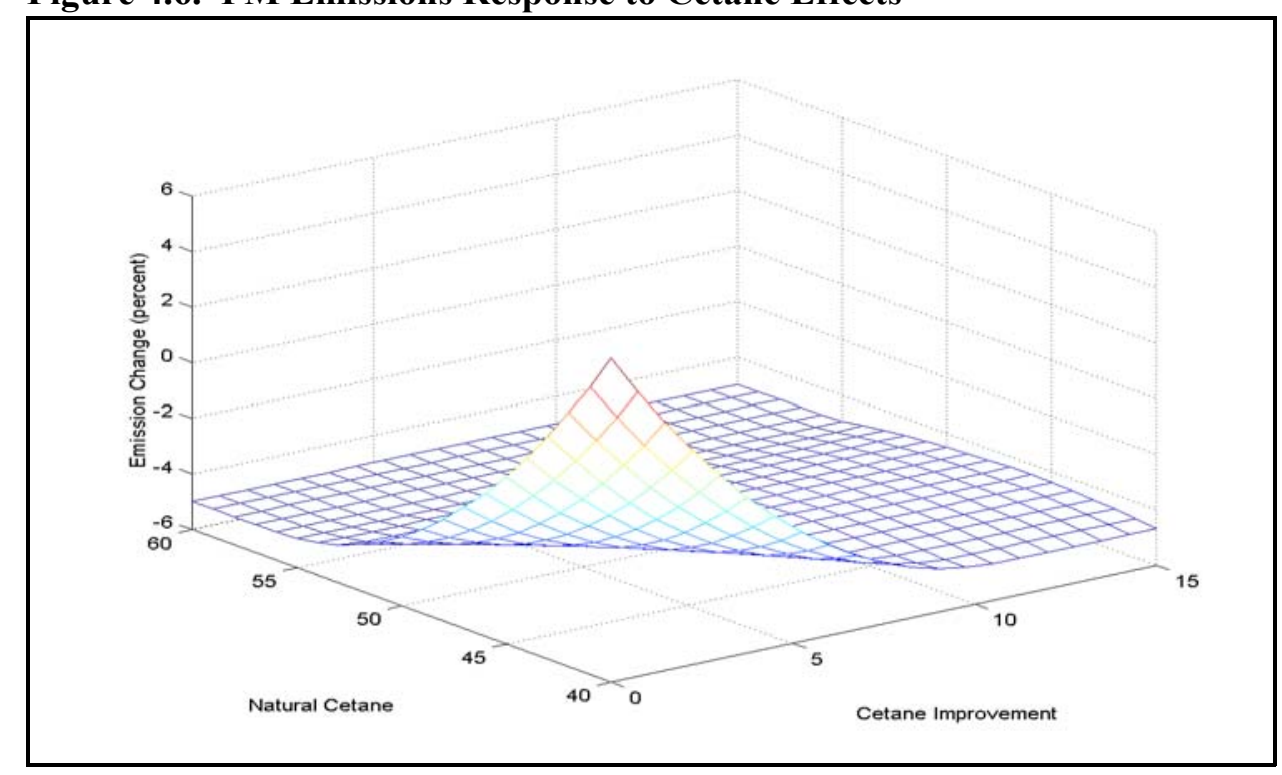

Figure 4.7 shows the trend in PM emissions with increasing total cetane. The solid line represents the effect of increasing natural cetane without use of cetane improvers. The dashed line represents cetane improvement of up to 15 numbers, by the use of additives with a base fuel of 45 natural cetane. PM emissions are reduced by approximately 5 percent when natural cetane number is raised to a total of 56 from the commercial average of 45, and is flat thereafter. Cetane improvement produces a somewhat more rapid reduction in PM emissions up to a saturation point at 52 total cetane ( 7 additized cetane numbers), but less total emissions reduction at higher numbers. The PM emissions effect of cetane improvers is largest in fuels with a natural cetane number below 50 as seen in Figure 4.8. Almost no effect remains when cetane additives are used in base fuels with natural cetane of 52 numbers or higher.

The PM emissions response to sulfur content is a 4-dimensional surface involving sulfur (linear and quadratic terms) and its interactions with aromatics content, specific gravity, and cetane improvement. Of these, the terms involving sulfur and aromatics content, shown in Figure 4.9, have the largest effects. Reducing the sulfur content of the average commercial fuel, with aromatics content of 33 percent, to below $15 \mathrm{ppm}$ is predicted to reduce PM emissions by only 3 percent (top line in the figure). The interaction with aromatics content potentiates this effect, however, increasing the emissions reduction as sulfur is removed from fuels of reduced aromatics content. As seen in the bottom line of the figure, decreasing the aromatics content to 5 percent, at constant sulfur content, is predicted to reduce PM emissions by nearly 10 percent. Removing sulfur from 5 percent aromatics fuel adds an additional 6 percent $P M$ emissions reduction when 15 ppm sulfur is reached, for a total of 16 percent reduction.

A similar, although smaller, effect occurs in the terms involving sulfur content and specific gravity, as shown in Figure 4.10. At a specific gravity of $0.850 \mathrm{gm} / \mathrm{cm}^{3}$ that is typical of current commercial fuels, PM emissions begin to decline when sulfur content is reduced below $250 \mathrm{ppm}$. The maximum reduction is 3 percent when sulfur content is decreased to $15 \mathrm{ppm}$. Decreasing the density of fuels to as low as 0.815 $\mathrm{gm} / \mathrm{cm}^{3}$ is predicted to reduce PM emissions by 1.8 percent, and the subsequent removal of sulfur to the level of 15 ppm adds an additional 4 percent. 
Figure 4.7. PM Emissions Response to Natural and Additized Cetane

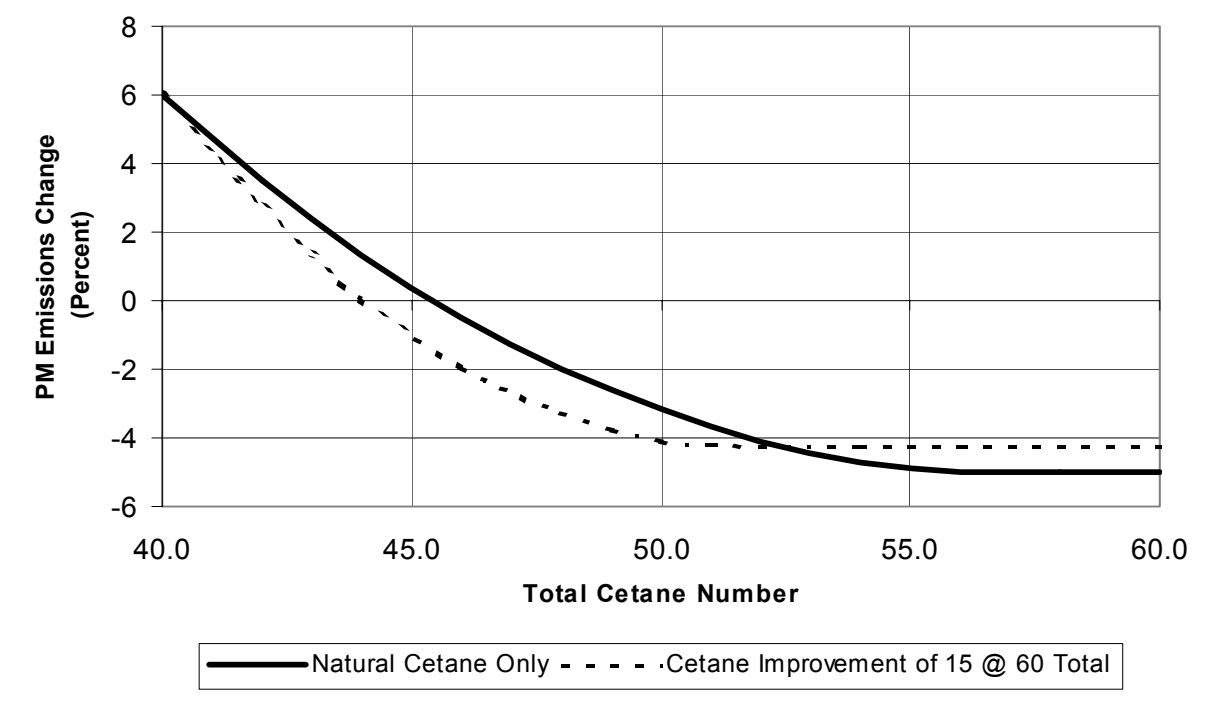

Figure 4.8. PM Emissions Response to Cetane Improvement

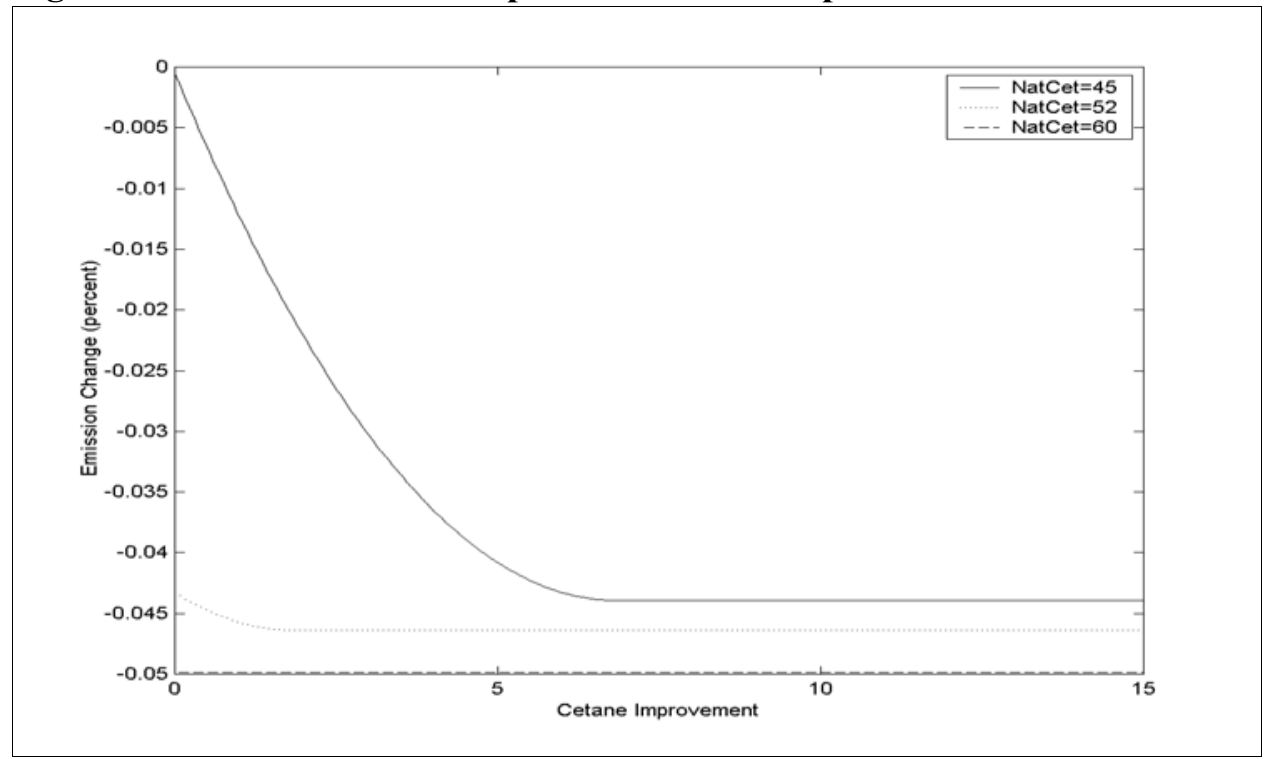


Figure 4.9. PM Emissions Response to Sulfur and Aromatics Content

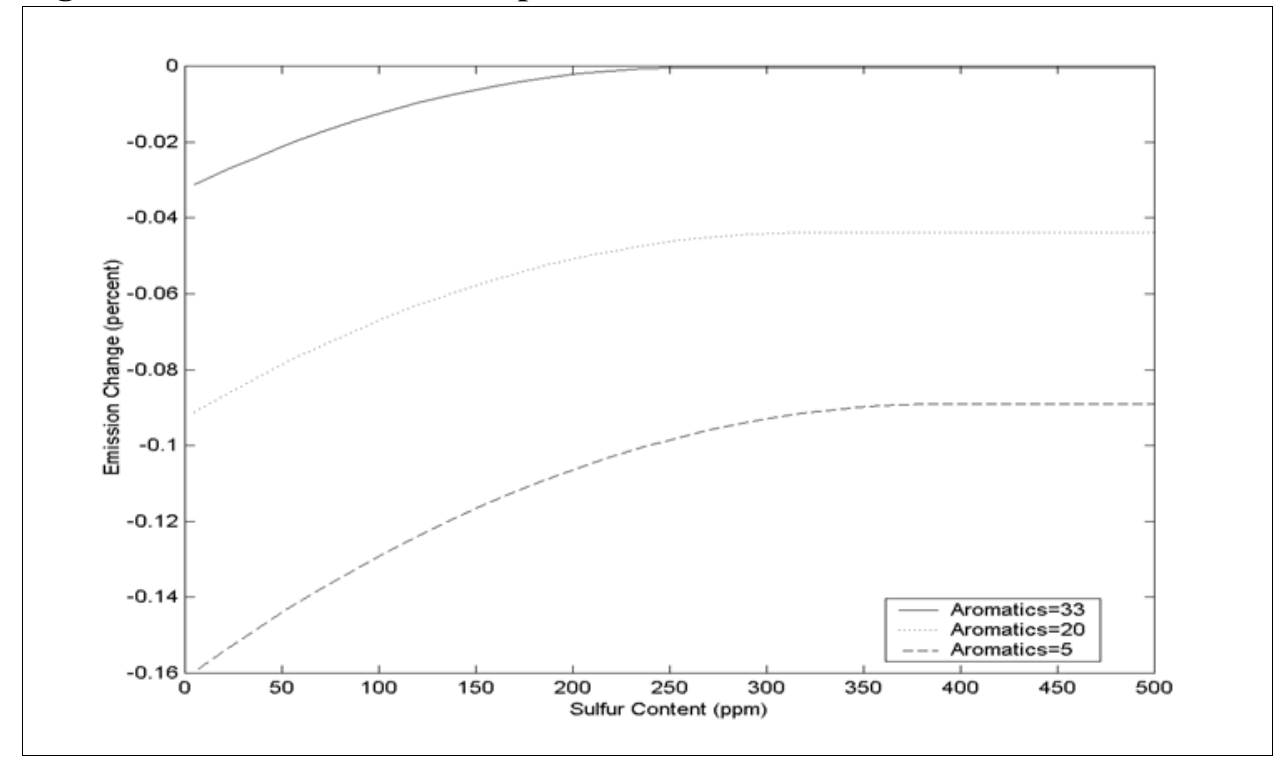

Figure 4.10. PM Emissions Response to Sulfur Content and Specific Gravity

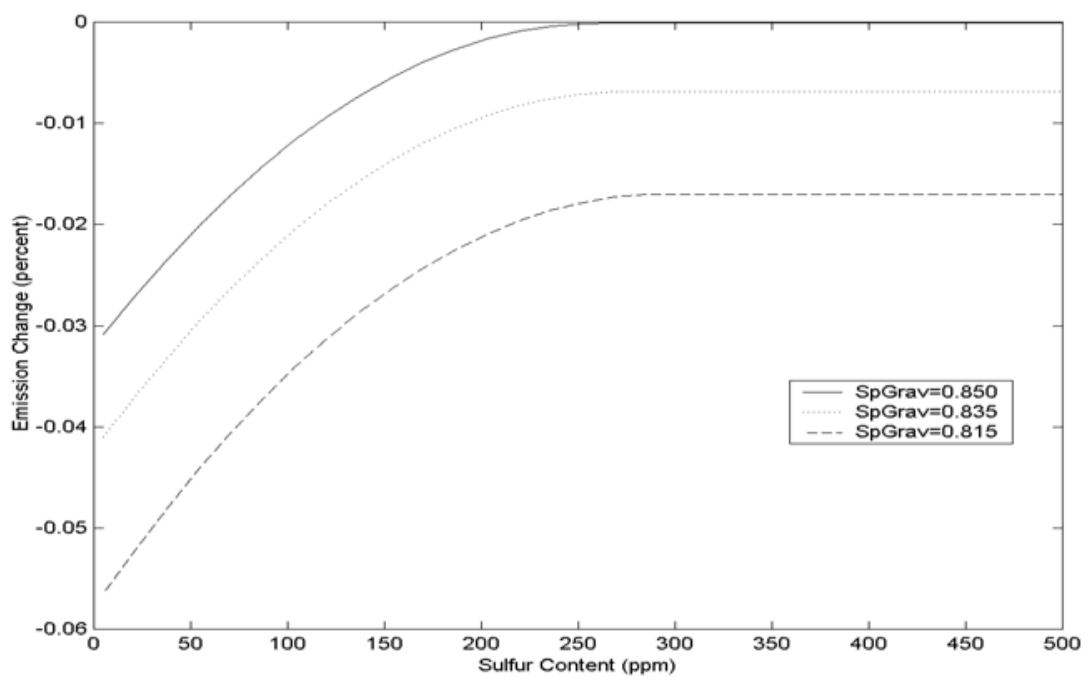

A similar potentiation of the benefit of reducing sulfur content also occurs in conjunction with cetane improvement, as shown in Figure 4.11. In current commercial fuels (top line of the figure), the benefit of reducing sulfur content to $15 \mathrm{ppm}$ is approximately 3 percent. An additional 4 percent PM reduction can be achieved by raising the cetane number 10 points through additives (lower line of the figure). Reducing sulfur content of the additized fuel to $15 \mathrm{ppm}$ then reduces PM emissions by an additional 7 percent, to reach a total of 11 percent reduction. 


\section{Figure 11. PM Emissions Response to Sulfur Content and Cetane}

Improvement

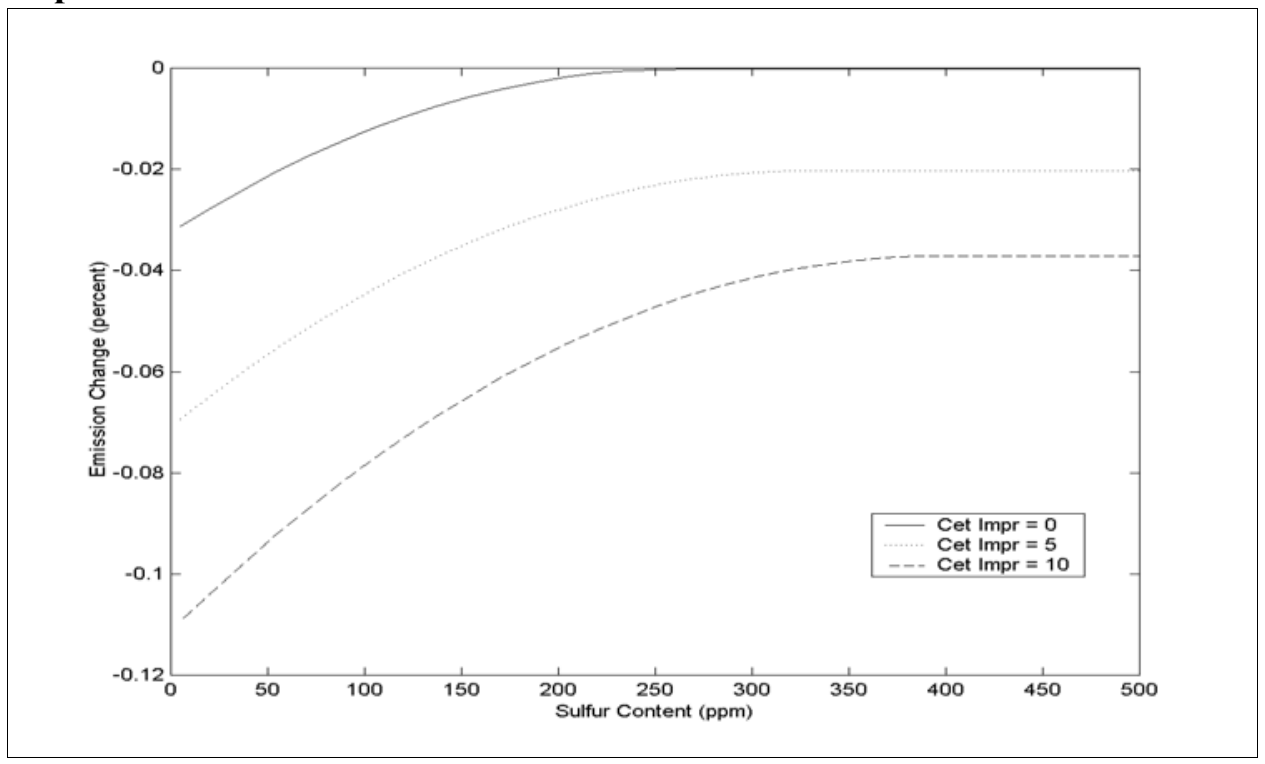

In general, one sees that the sulfur terms in the PM emissions model indicate increased benefits from sulfur reduction in fuels that have been improved, compared to current commercial fuels, in terms of aromatics content, specific gravity, or additized cetane. To our knowledge, such effects have not been explored in prior research and are not well-understood in terms of combustion processes. Nevertheless, the effects clearly indicate that multi-property fuel improvements along these axes tend to potentiate each other in reducing PM emissions.

In addition to cetane and sulfur content, oxygen is predicted to have a large effect on PM emissions. PM emissions are predicted to decline by 25 percent when oxygen content reaches 5 percent (by weight). The trend shows a modest degree of acceleration as oxygen content increases.

Table 4.5 summarizes the sensitivity of emissions to changes in the fuel property values. If emissions are decreased by raising the property value, the columns to the right of center show the maximum PM reduction when the property is moved to the upper end of the relevant range. If emissions are decreased by reducing the property value, the columns to the left of center show the maximum PM reduction when the property is moved to the lower end of the relevant range.

Clearly, the path to PM emissions reduction lies in adding oxygen to fuels and in reducing sulfur content in conjunction with aromatics. Total cetane and cetane improvement make smaller, but still substantial contributions to PM emissions reductions, while other properties and the distillation curve appear to have much smaller effects.

The RYM model predicts an 11.3 percent reduction in PM emissions for the average diesel fuel found in the Los Angeles Basin. This fuel is one in which aromatics content has been reduced to 22 percent, the sulfur content cut to $130 \mathrm{ppm}$, and the total cetane number raised to 52. The predicted emission change is nearly twice the 6.9 percent reduction estimated using a simple, linear eigenvector model in McAdams 2002. That PM estimate was caveated, however, because the prior eigenfuel model did not consider interaction terms that EPA's analysis had indicated were important. Now that quadratic and interaction terms are considered, the 
current estimate is now similar to, and somewhat greater than, the 8.9 percent reduction estimated by EPA's analysis.

Table 4.5. Magnitude of Predicted PM Emissions Response

\begin{tabular}{lcccccc}
\hline & & $\begin{array}{c}\text { Property } \\
\text { Value }\end{array}$ & $\begin{array}{c}\text { Emissions } \\
\text { Change } \\
\text { (Percent) }\end{array}$ & $\begin{array}{c}\text { Average } \\
\text { Commercial } \\
\text { Fuel }\end{array}$ & $\begin{array}{c}\text { Property } \\
\text { Value }\end{array}$ & $\begin{array}{c}\text { Emissions } \\
\text { Change } \\
\text { (Percent) }\end{array}$ \\
\hline TotCetane & number & & & 45.4 & 60 & -5.0 \\
CetImprv & number & & & 0 & 15 & -5.5 \\
Density & ${\mathrm{gm} / \mathrm{cm}^{3}}^{3}$ & 0.815 & -1.7 & 0.850 & & \\
Viscosity & $\mathrm{mm}^{3} / \mathrm{sec}$ & & & 2.67 & 3.25 & -0.2 \\
Sulfur & $\mathrm{ppm}$ & 5 & -2.9 & 352 & & \\
Aromatics & vol \% & 5 & -9.0 & 33.2 & & \\
IBP & deg F & 175 & -1.6 & 349 & & \\
T10 & deg F & & & 429 & 465 & -0.0 \\
T50 & deg F & & & 513 & 540 & -0.2 \\
T90 & deg F & 520 & -1.9 & 607 & & \\
FBP & deg F & 590 & -1.1 & 653 & & \\
Oxygen & wt \% & & & 0 & 5 & -23.9 \\
\hline
\end{tabular}





\section{II-5. IMPLEMENTATION IN RYM}

\section{II-5.1 REPRESENTING TECHNOLOGICAL CHANGE}

At any point in time, the emissions effect of reformulating diesel fuel will depend on the mix of engine technologies on the road, the baseline exhaust emissions of each technology type, and the response of emissions to changes in fuel properties, which could vary by technology type. There will soon be substantial changes in diesel engines and fuels as the 2004 exhaust emission standards are implemented. After that point, new engines are expected to have EGR systems, oxidation catalysts, and particulate traps, and the sulfur content of diesel fuel will be cut to $10-15 \mathrm{ppm}$ as an enabling measure to protect emission control systems. A key issue in this analysis is how a database that pertains almost entirely to current engines, without EGR or exhaust after-treatment, can be used to develop emission models representing the progressively changing fleet of vehicles between now and 2010.

As a starting point, differences in the emissions response to fuels, measured on a percentage basis, among engine technology groups in the current on-road fleet appear to be small. The EPA analysis detected modest differences in the response to fuels for several groups, but the sales-weighted influence of these groups was seen to be of essentially no importance. EPA's conclusion was that, for the current population of on-road engines, the emissions response to fuel changes appeared to be homogeneous. Therefore, we may treat the current fleet of on-road engines as a single group, as in the statistical models presented in the prior section.

The emissions responses estimated through the statistical analysis are used here as benchmark functions that are tied to the technology level and fuel-responsiveness of current on-road engines. These functions are directly representative of the emissions response for current engines that survive to future years, and they are used with adjustments to estimate the emissions response in newer technology engines equipped with EGR, exhaust after-treatment devices, or advanced engine combustion controls.

EPA estimates that EGR-equipped engines will account for 45 percent of the diesel on-road $\mathrm{NO}_{\mathrm{x}}$ inventory by 2010 and 64 percent by 2020 (see Table 5.1). Only one study (HDEWG 1999) has tested an EGRequipped engine on a range of fuels. Its conclusion was that fuel cetane appears to have little or no effect on $\mathrm{NO}_{\mathrm{x}}$ emissions, at least for the engine configuration tested. This result could be understood as suggesting that EGR reduces peak combustion temperatures and pressures in a way that is similar to the combustion changes introduced by increasing the cetane number. Having modified combustion characteristics through the use of EGR, there appears to remain essentially no additional benefit that can be achieved by increasing cetane.

To represent this effect, the cetane terms in the $\mathrm{NO}_{\mathrm{x}}$ model are adjusted downward over time in proportion to the declining number of non-EGR engines operating in fleet. By 2010, the most distant time period to be considered in RYM studies, the $\mathrm{NO}_{\mathrm{x}}$ benefit of increasing cetane number will be reduced to 55 percent of its current value to reflect the proportion of the year 2010 fleet without EGR. The emissions response to other fuel property changes, and of PM emissions to fuels, remain the same, in relative terms, as for the current fleet.

The implication of measuring emission changes on a relative basis is that the absolute change in mass emissions - whether measured as grams per mile or grams per brake-horsepower-hour - will be smaller in the future as the baseline emission factors for the overall fleet decline. That is, although we assume that the emission changes on a fractional or percentage basis predicted for current engines will apply equally to future engines, the lower baseline emission rate of the future engines will translate into a smaller total effect of mass emissions. 
Table 5.1. Fraction of Diesel Highway $\mathrm{NO}_{x}$ Inventory

by EGR System Type

(percent of fleet)

\begin{tabular}{ccc}
\hline Year & Non-EGR & EGR \\
\hline 2002 & 100 & 0 \\
2003 & 93 & 7 \\
2004 & 84 & 16 \\
2005 & 77 & 23 \\
2006 & 70 & 30 \\
2007 & 65 & 35 \\
2008 & 61 & 39 \\
2009 & 57 & 43 \\
2010 & 55 & 45 \\
2015 & 48 & 52 \\
2020 & 36 & 64 \\
\hline
\end{tabular}

Source: EPA 2002, Table III.B-1

Having adjusted the $\mathrm{NO}_{\mathrm{x}}$ / cetane relationship for the effect of EGR, we believe it is reasonable to assume, absent advances in engine combustion control, that the resulting functions can be used to estimate the relative emissions change for engines with exhaust catalysts and/or particulate traps. Without advances in engine combustion control, the adjusted emissions response should be a reasonable surrogate for the engine out emission levels of future engines equipped with after-treatment devices. Because fuels effects on emissions are expected to be modest (in the range of 5 to 10 percent for $\mathrm{NO}_{\mathrm{x}}$ and 10-20 percent for PM), they are unlikely to have an appreciable effect on the removal efficiency of after-treatment devices. Thus, the percentage changes in engine-out emissions should pass through to equal percentage changes in exhaust emissions, although the exhaust change will be referenced to a much lower emission baseline.

This assumption is supported by work performed for ORNL (Duleep 2001a,b), which examined test data on light-duty diesel vehicles equipped with prototype advanced emission control systems. In general, the study concluded that the percentage response of exhaust emissions to fuel changes was as large, or perhaps larger, in these vehicles as in their predecessors without after-treatment. However, a percentage reduction of given size implies much less effect on mass emissions when baseline emission levels have already been reduced by as much as 80 percent by after-treatment devices.

In response to the 2004 standards, engine manufacturers are expected to make a number of advances in controlling emission formation during the combustion process by methods such as advanced combustion chamber design and sophisticated computerized control of injector operation. As in the example of EGR, these advances may exploit combustion changes that, in current engines, are the avenue by which fuel reformulation reduces emissions. If a shift in combustion takes place through combustion control, there will likely be less benefit to fuel reformulation in future engines.

With future engine designs still in development, and essentially no test data in the public forum, the potential future effects of engine combustion control can only be represented in terms of bracketing scenarios. EGR appears to eliminate the sensitivity to one fuel parameter, while not substantially changing the sensitivity to 
other factors. The reality for engine controls may proved to be just as complex, although only "broad brush" assumptions can be made at this time. For purposes of RYM studies, the bracketing scenarios are:

- Scenario 1 in which engine controls are assumed to reduce the sensitivity to fuels by an appreciable amount, but not to the extent of eliminating the response. A reduction of onethird in the emissions response has been chosen (arbitrarily) to represent this bound.

- Scenario 2 in which engine controls are assumed to eliminate the sensitivity of emissions to fuels (all parameters) in future engines. This bound obviously represents the greatest effect that engine combustion controls could have on the potential benefits of fuel reformulation.

These effects will occur only in engines produced in 2004 and later that are designed with advanced engine combustion controls. In discussions with ORNL staff, personnel from the American Petroleum Institute (API) estimated that as much as 25 to 30 percent of the on-road fleet could carry such controls by 2010 . Table 5.2 shows the assuming timing for the introduction of advanced engines in the fleet, and their effect in reducing the fleetwide sensitivity to fuels changes in the two scenarios. By 2010, the presence of advanced engines would reduce the emissions response to fuels (after EGR adjustment) by 10 percent under the first scenario and by 30 percent under the second scenario.

Table 5.2. Assumptions for the Effect of Advanced Technology Engines on Fleetwise Emissions Response to Fuels

\begin{tabular}{ccccc}
\hline & \multicolumn{2}{c}{ Composition of Fleet } & \multicolumn{2}{c}{ Fleetwide Fuels Sensitivity } \\
\cline { 2 - 5 } Year & $\begin{array}{c}\text { Conventional } \\
\text { Engines: with or } \\
\text { without EGR } \\
\text { (percent) }\end{array}$ & $\begin{array}{c}\text { Advanced } \\
\text { Technology } \\
\text { Engines } \\
\text { (percent) }\end{array}$ & $\begin{array}{c}\text { Scenario 1: One- } \\
\text { third less Fuels } \\
\text { Effect } \\
\text { (percent) }\end{array}$ & $\begin{array}{c}\text { Scenario 2: Fuels } \\
\text { Effect Eliminated }\end{array}$ \\
\hline 2003 & 100 & 0 & 100 & 100 \\
2004 & 95 & 5 & 98 & 95 \\
2005 & 89 & 11 & 96 & 89 \\
2006 & 84 & 16 & 95 & 84 \\
2007 & 79 & 21 & 93 & 79 \\
2008 & 76 & 24 & 92 & 76 \\
2009 & 73 & 27 & 91 & 73 \\
2010 & 70 & 30 & 90 & 70 \\
\hline
\end{tabular}

The effect of advanced engines in reducing the fleetwide sensitivity to fuels will be accounted for in ex post calculations of fleetwide emissions benefits, rather than by direct implementation in RYM. The emissions functions in RYM will estimate emission changes before considering the potential range of effects due to advanced technology engines. For example, model results from a hypothetical case study might estimate that a particular fuel reformulation would reduce $\mathrm{NO}_{\mathrm{x}}$ emissions by 5 percent and $\mathrm{PM}$ emissions by 15 percent in 2010, after considering the effects of EGR-equipped engines. The case study would report that, after considering the effects of advanced technology engines, the specified fuel would reduce $\mathrm{NO}_{\mathrm{x}}$ by amounts ranging from 3.5 to 4.5 percent (70 to 90 percent of the nominal 5 percent change) and PM by amounts ranging from 10.5 to 13.5 percent ( 70 to 90 percent of the nominal 15 percent change). 


\section{II-5.2 IMPLEMENTATION IN RYM}

The emissions models are implemented in RYM by pre-evaluating the equations for each blendstock in the model to create $\mathrm{NO}_{\mathrm{x}}$ and $\mathrm{PM}$ "blending values". The blending values are, in concept, estimated contributions of each blendstock to the emissions level of finished fuels in which the blendstocks are used. The RYM solution process will assume that the blending values combine linearly according to the volume fractions in the final fuel. The full emission equations will be evaluated after the model's solution and the precise value of the predicted emissions response compared with that estimated by RYM under linear blending. If the difference is large, the RYM case will be re-run.

The degree of nonlinearity in the equations is generally small across the range of fuel modifications that are likely to be encountered in case studies. The primary exception to this generality is the cetane surface for PM emissions, in which the emissions response slows rapidly as total cetane number is increased and reaches saturation at a total cetane of 56. A Monte Carlo simulation was conducted to put bounds on the likelihood of divergence. A total of 1000 synthetic fuels was generated by uniform, random sampling within the range of relevant fuel property values identified in Table 4.1. $\mathrm{NO}_{\mathrm{x}}$ and $\mathrm{PM}$ blending value were computed for each fuel using the emissions equations in Tables 4.2 and 4.4. The 1000 fuels were then treated as blendstocks to create new fuels.

The blendstock simulation selected 2 fuels at random and blended them according to random weights that totaled 100 percent. The individual fuel properties were blended linearly, as were the $\mathrm{NO}_{\mathrm{x}}$ values, to produce a simulated final fuel. A total of 100 such "final fuels" were generated. Some fuel properties, such as aromatics, will blend linearly, while other properties will not. The greatest non-linearity in blending may lie in the distillation temperatures. However, these properties have relatively little effect on emissions and efforts to obtain nonlinear blending algorithms did not yield reliable relationships. Thus, linear blending was used as a reasonable approximation, but it will tend to understate the degree of divergence to be encountered in RYM.

The results of the simulation give us reason to believe that pre-evaluating the equations for all blendstocks will be a satisfactory approach for implementing the equations in RYM. Of 100 cases generated, most of the errors in estimate are within 1 part in 10 of the precisely calculated emission response. This means that a 5 percent in reduction in $\mathrm{NO}_{\mathrm{x}}$ would be estimated to within $+/-0.5$ percent by the model (4.5 to 5.5 percent), while a 20 percent reduction in PM would be estimated to within $+/-2$ percent by the model ( 18 to 22 percent).

It must be recognized that the actual degree of divergence between the RYM solution and the precisely calculated value are likely to be greater than shown in the simulation. First, RYM employs nonlinear blending algorithms for properties such as sulfur content and the distillation curve that do not blend in accordance with volume weights. The divergence estimated in the simulation would increase if nonlinear blending were allowed. Second, the actual blendstocks in the RYM model span a wider range in fuel properties than was considered in the simulation. For example, a series of blendstocks will represent nonaromatic feeds and, therefore, have zero aromatics content; other blendstocks may represent aromatic feeds and, therefore, have 100 percent aromatics content. In the simulation, the hypothetical "blendstocks" ranged between 5 and 42 percent. As a result, blendstocks in the model are more likely to fall in the range of values where the emissions response saturates, thereby increasing the divergence between RYM solution and the precise emissions response. Nevertheless, the divergence can be reduced whenever necessary by rerunning a case. 


\section{REFERENCES}

Department of Energy. 1994. Estimating the Costs and Effects of Reformulated Gasoline. DOE/PO0030. December.

Duleep, K.G. 2001. Diesel Technology and Fuel Requirements for Low Emissions: Phase I, Energy and Environmental Analysis, Inc., under contract with ORNL, May.

Duleep, K.G. 2001. Diesel Technology and Fuel Requirements for Low Emissions, Energy and Environmental Analysis, Inc., under contract with ORNL, May.

Environmental Protection Agency. 2001. Strategies and Issues in Correlating Diesel Fuel Properties with Emissions: Staff Discussion Document. EPA420-P-01-001. July.

Environmental Protection Agency. 2002. The Effect of Cetane Number Increase Due to Additives on $\mathrm{NO}_{x}$ Emissions from Heavy-Duty Highway Engines. EPA420-S-02-012. June.

Grabowski, M.S., R.L. McCormick, T.L. Alleman, A.M. Herring. 2000. The Effect of Biodiesel Composition on Engine Emissions From a DDC Series 60 Diesel Engine. Colorado Institute for Fuels and Engine Research, Colorado School of Mines, under contract with NREL, June.

Heavy Duty Engine Working Group (HDEWG). 1999. Phase II Briefing for Meeting of the Mobile Sources Technical Review Subcommittee. Clean Air Act Advisory Committee. Washington, D.C. January 13.

McAdams, H.T., R.W. Crawford and G.R. Hadder. 2000. A Vector Approach to Regression Analysis and Its Application to Heavy-Duty Diesel Emissions. SAE 2000-01-1961.

McAdams, H.T., R.W. Crawford and G.R. Hadder. 2000. A Vector Approach to Regression Analysis and Its Application to Heavy-Duty Diesel Emissions. ORNL/TM-2000/5.

McAdams, H.T., R.W. Crawford and G.R. Hadder. 2001. PCR + in Diesel Fuels and Emissions Research. ORNL/TM-2002/16.

Southwest Research Institute. 2001. Diesel Fuel Impact Model Data Analysis Plan Review. SwRI 08.04075 . 



\section{APPENDIX II-A}

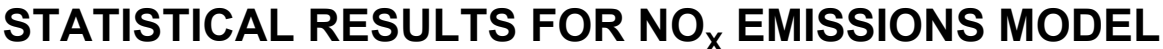



Table A.1. PCA Decomposition of Experimental Fuels for $\mathrm{NO}_{\mathrm{x}}$ Model

\begin{tabular}{|c|c|c|c|c|c|c|c|c|c|c|c|c|c|c|c|c|c|}
\hline \multicolumn{17}{|c|}{ PCA Eigen variables } & 17 \\
\hline TotCetane & 0.107 & 0.474 & 0.175 & -0.200 & 0.057 & -0.426 & 0.141 & -0.080 & 0.123 & -0.129 & 0.225 & -0.159 & 0.140 & -0.241 & 0.253 & -0.350 & 0.33 \\
\hline CetImprv & 0.029 & -0.242 & 0.418 & 0.414 & -0.025 & -0.406 & 0.075 & -0.145 & 0.120 & -0.290 & -0.193 & 0.128 & -0.006 & 0.287 & -0.143 & 0.294 & -0.246 \\
\hline Density & 0.312 & 0.176 & 0.048 & 0.443 & -0.080 & 0.081 & 0.049 & 0.039 & 0.067 & -0.001 & -0.358 & -0.030 & -0.426 & -0.032 & 0.439 & -0.351 & 0.156 \\
\hline Viscosity & 0.395 & -0.099 & -0.154 & 0.053 & 0.042 & 0.001 & 0.073 & 0.182 & 0.202 & 0.173 & -0.038 & 0.007 & -0.030 & 0.102 & -0.212 & 0.487 & 0.634 \\
\hline Sulfur & 0.268 & 0.045 & 0.191 & -0.217 & 0.285 & 0.272 & 0.135 & -0.449 & -0.196 & -0.189 & -0.278 & -0.529 & -0.002 & -0.000 & -0.188 & 0.043 & 0.005 \\
\hline Aromati & 210 & 0.182 & 106 & 0.494 & -0.272 & 0.114 & 0.028 & -0.298 & -0.290 & 0.026 & 03 & 0.032 & & -0.236 & -0.093 & -0.019 & 0.138 \\
\hline IBP & 0.223 & -0.025 & -0.365 & 0.081 & 0.322 & -0.118 & 0.016 & 0.330 & -0.510 & -0.461 & 0.045 & 0.038 & 0.163 & 0.144 & 0.223 & 0.072 & -0.061 \\
\hline T10 & 0.362 & 0.091 & -0.178 & 0.159 & 0.264 & -0.109 & 0.062 & 0.148 & 0.128 & 0.195 & 0.098 & 0.019 & -0.068 & -0.032 & -0.578 & -0.454 & -0.294 \\
\hline T50 & 0.394 & 0.124 & -0.028 & -0.044 & -0.052 & 0.076 & 0.121 & 0.039 & 0.203 & 0.261 & 0.252 & -0.217 & 0.012 & -0.112 & 0.410 & 0.354 & -0.52 \\
\hline T90 & 0.3 & 0.1 & $-0 . c$ & -0.244 & 0. & 0.029 & -0.2 & -0 & -0.044 & 0.0 & -0 . & & & & 0.047 & -0.253 & -0.017 \\
\hline FBP & 0.299 & -0.128 & -0 & -0.2 & 0. & -0.044 & -0.209 & -0 & -0.108 & -0.298 & -0 . & 0. & -0.224 & -0.508 & -0.172 & 0.111 & -0.113 \\
\hline Oxygen & -0.011 & 0.004 & 0.300 & -0.104 & -0.367 & 0.219 & 0.647 & 0.474 & -0.142 & -0.134 & 0.047 & -0.051 & -0.016 & 0.027 & -0.153 & -0.071 & -0.016 \\
\hline Tot Cet ^ 2 & -0.155 & -0.213 & -0.360 & 0.072 & -0.106 & -0.232 & 0.450 & -0.267 & -0.357 & 0.437 & -0.350 & 0.061 & & -0.064 & 0.033 & 0.045 & -0.03 \\
\hline Sulfur $\wedge 2$ & -0.221 & -0.035 & -0.417 & 0.238 & -0.356 & -0.108 & -0.091 & 0.113 & 0.234 & -0.256 & -0.078 & -0.636 & 0.062 & -0.084 & -0.132 & 0.019 & -0.03 \\
\hline Aromatics`2 & -0.081 & -0.281 & -0.343 & 0.095 & 0.046 & 0.450 & 0.309 & -0.351 & 0.313 & -0.342 & 0.194 & 0.289 & -0.084 & 0.128 & 0.042 & -0.056 & 0.01 \\
\hline CImp $\times$ Dens & -0.127 & -0.498 & & 0.140 & -0.055 & & -0.255 & & -0.410 & & & -0.185 & -0.529 & 0.117 & -0.071 & & 0.04 \\
\hline CImp $\times$ Arom & -0.058 & -0.461 & 0.139 & 0.174 & 0.112 & 0.424 & -0.218 & 0.260 & 0.047 & 0.096 & -0.403 & 0.051 & 0.455 & -0.196 & 0.045 & -0.051 & -0.03 \\
\hline Eigenvalues & 5.322 & 2.422 & .805 & 1.532 & 1.210 & 1.058 & 0.967 & 0.793 & 0.542 & 0.493 & 0.260 & 0.216 & 0.141 & 0.089 & 0.069 & 0.060 & 0.02 \\
\hline & & & & & & & & & & & & & & & & & \\
\hline Cumulative Pct & 1.307 & 45.554 & 56.172 & 5.184 & 72.303 & 8.525 & 84.214 & 88.881 & 92.067 & 94.967 & 96.497 & 97.767 & 98.595 & 99.120 & 99.528 & 99.879 & 0 . \\
\hline
\end{tabular}

PCA Eigen variables in physical units

\begin{tabular}{|c|c|c|c|c|c|c|c|c|c|c|c|c|c|c|c|c|c|}
\hline & 1 & 2 & 3 & 4 & 5 & 6 & 7 & 8 & 9 & 10 & 11 & 12 & 13 & 14 & 15 & 16 & 17 \\
\hline & 0.674 & 2.971 & 1.097 & -1.257 & 0.356 & 2.673 & 35 & 9 & 等1 & -0 . & & -0 . & & & 1.583 & & \\
\hline etImp & 0.130 & 1.085 & .874 & 1.857 & -0.111 & 1.821 & 338 & -0.6 & 0.540 & -1.301 & -0.865 & & -0.028 & 286 & -0.641 & 1.318 & -1.102 \\
\hline ensity & 0.005 & 0.003 & 0.001 & 0.007 & -0.001 & 0.001 & .001 & 0.001 & 0.001 & -0.000 & -0.005 & -0.000 & -0.006 & -0.000 & 0.007 & -0.005 & 0.002 \\
\hline$s c$ & 0.249 & -0.062 & -0.097 & 0.033 & 0.026 & 01 & 46 & 0.115 & 0.127 & & & & & & -0.133 & & \\
\hline ulf & 13.943 & 7.390 & 31.388 & -35.671 & 46.711 & 44.631 & 2.222 & -73.622 & -32.088 & -30.944 & -45 & -86.757 & -0.323 & -0.016 & -30.775 & 83 & 0.866 \\
\hline :om & 1.716 & 1.488 & 0.865 & 4.040 & -2.220 & 0031 & 0.228 & -2.432 & -2.368 & & & 0.258 & & & -0.759 & -0 . & 1.124 \\
\hline $3 P$ & 2.321 & -1.366 & -20.173 & 4 & $17 . \varepsilon$ & -6. & 0.891 & 18.253 & -28.221 & -25 & & 79 & 13 & 77 & 12.309 & 3.964 & -3.373 \\
\hline 10 & .016 & 3.506 & -6.8 & 6.1 & 10.225 & -4.208 & 2.391 & 5.741 & 4.943 & & & & -2.641 & & -22.374 & -17 & -11.385 \\
\hline 50 & .363 & -4.2 & -0.9 & -1.493 & -1.761 & 2.5 & 4.095 & 1.325 & 6.891 & & & -7 & 0. & 13 & 15 & 29 & -17.830 \\
\hline 0 & 5 & 4. & -2 . & -8.515 & -16.066 & & 1 & -3. & -1.5 & & & & & & & & \\
\hline 3P & 9.986 & -4.2 & -3.5 & -9.016 & -13.137 & -1.4 & -6.5 & -2.290 & -3.614 & & & & -7 . & 37 & 33 & & 754 \\
\hline xyge & -0.005 & 0.002 & 0.136 & -0.047 & -0.166 & 0.099 & 0.293 & 0.215 & -0.064 & -0 . & 21 & -0 . & -0.007 & 12 & -0.069 & -0.032 & -0.007 \\
\hline & 0.244 & -0.336 & -0.5 & & -0.1 & -0.3 & & -0.420 & -0.562 & & & & & & & & \\
\hline & 0.251 & -0.040 & -0 . & & -0. & -0.3 & -0.1 & & 0.2 & & & -0 & & & 150 & 21 & 035 \\
\hline omatics^2 & 0.114 & -0.393 & -0.480 & 0.1 & 0.065 & 0.629 & 0.432 & -0.491 & 0.438 & -0.479 & 72 & 0.405 & -0.117 & 79 & 0.059 & -0.079 & 0.016 \\
\hline$m p \times$ Dens & 1 & -0.410 & & & -0.045 & & & & -0.3 & & & & & & & & .037 \\
\hline Imp $\times$ Arom & -0.052 & -0.414 & 0.125 & 0.156 & 0.101 & 0.382 & -0.196 & 0.233 & 0.042 & 0.086 & -0.362 & 0.046 & 0.409 & -0.176 & 0.041 & -0.046 & -0.029 \\
\hline
\end{tabular}


Table A.2. PCR+ Regression Model for $\mathrm{NO}_{\mathrm{x}}$ Emissions

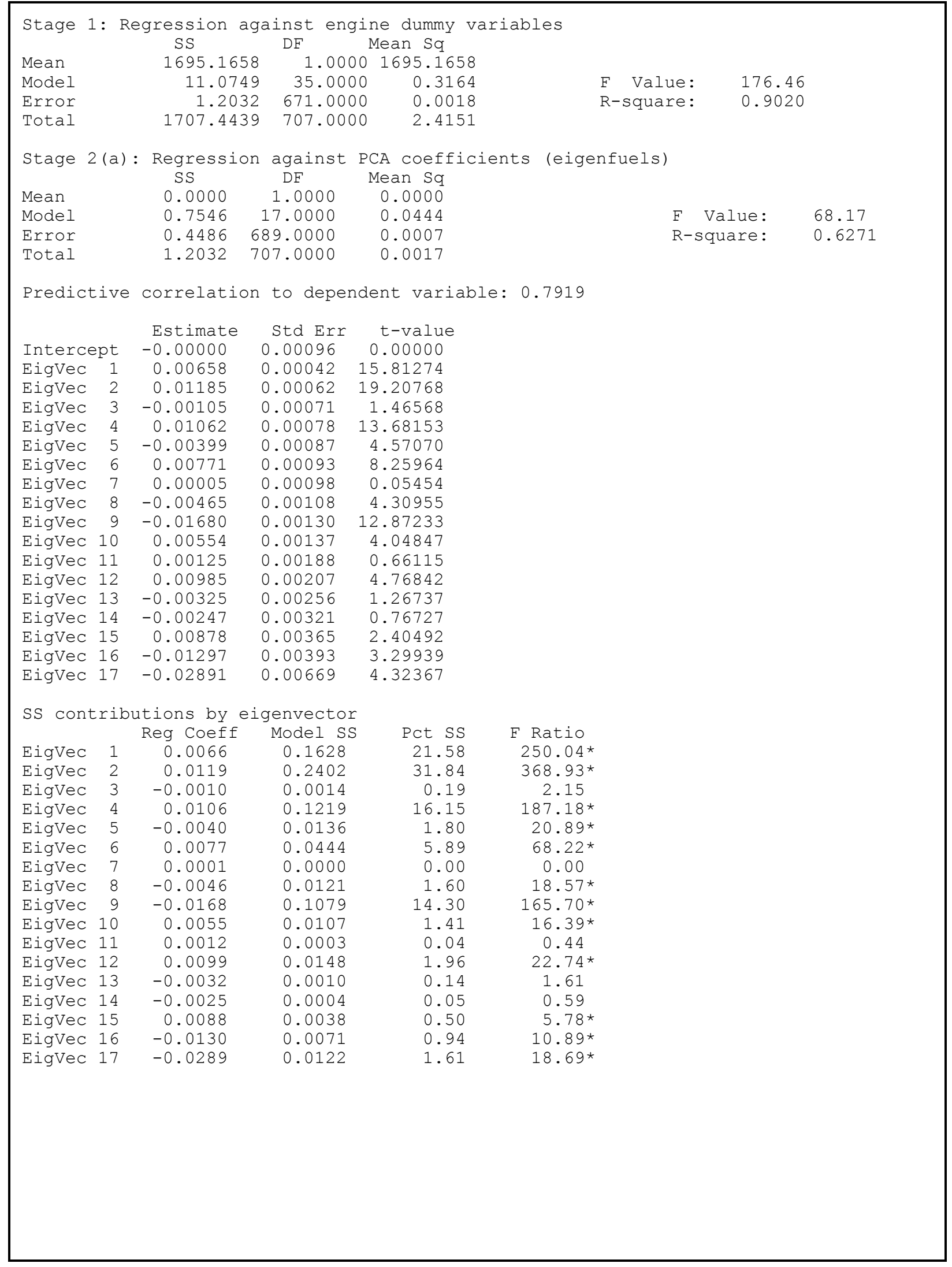


Table A.2. (Continued)

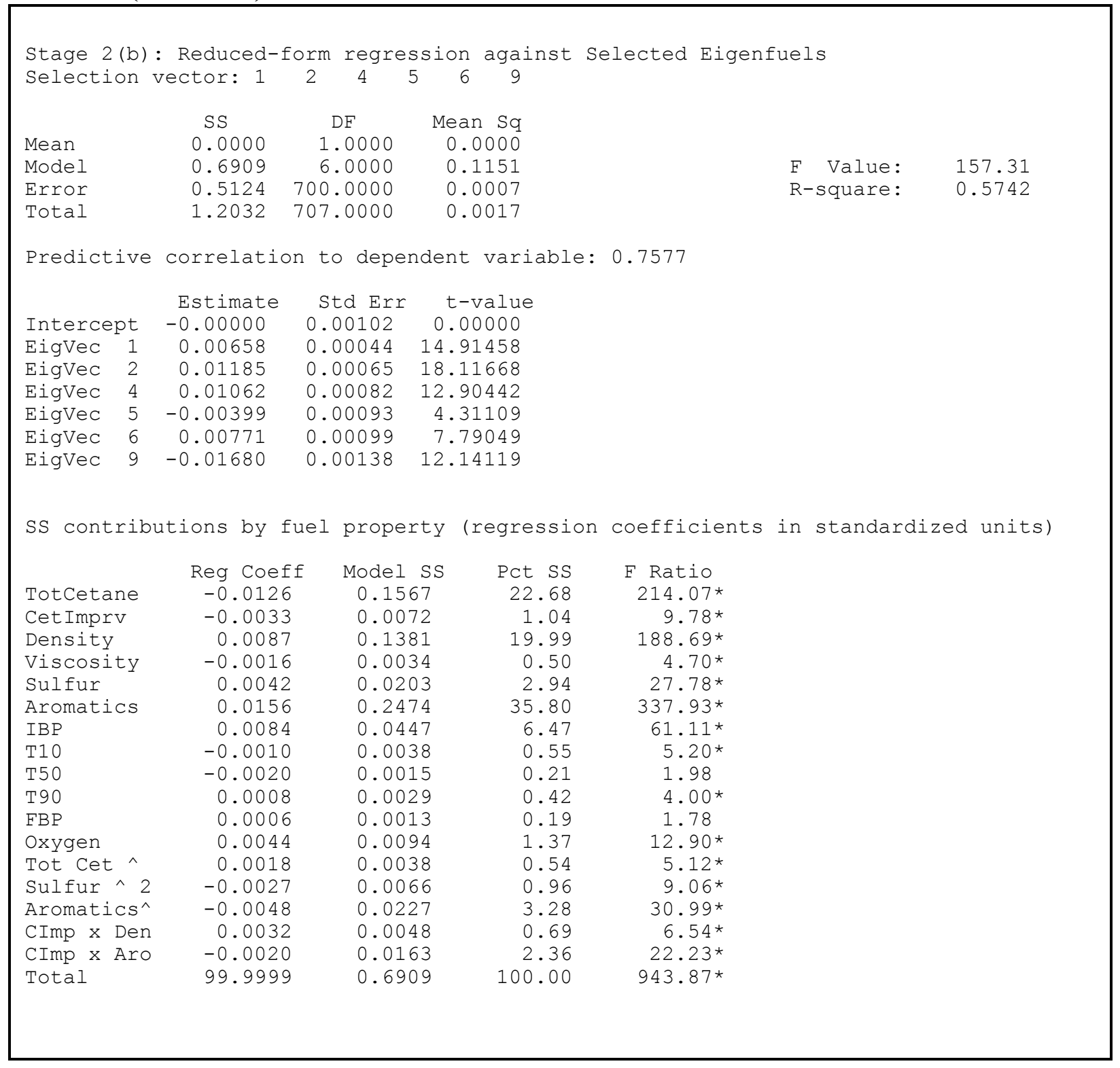



APPENDIX II-B

STATISTICAL RESULTS FOR PM EMISSIONS MODEL 

Table B.1. PCA Decomposition of Experimental Fuels for PM Model

\begin{tabular}{|c|c|c|c|c|c|c|c|c|c|c|c|c|c|c|c|c|c|c|c|}
\hline \multicolumn{20}{|c|}{ PCA Eigen variables } \\
\hline TotCetane & 0.125 & -0.080 & 0.169 & -0.144 & 0.664 & -0.059 & 0.014 & -0.169 & 0.098 & -0.003 & -0.126 & -0.157 & 0.028 & 0.271 & 0.129 & -0.378 & 0.183 & -0.117 & -0.353 \\
\hline CetImprv & 0.048 & 0.212 & -0.175 & -0.462 & 0.381 & -0.027 & 0.171 & -0.219 & 0.259 & 0.115 & -0.193 & -0.014 & -0.141 & -0.281 & -0.215 & 0.348 & -0.129 & 0.084 & 0.280 \\
\hline Density & 0.308 & 0.121 & -0.207 & -0.226 & -0.331 & -0.002 & -0.004 & -0.057 & 0.028 & -0.069 & 0.042 & -0.194 & -0.413 & -0.368 & -0.013 & -0.355 & 0.394 & 0.087 & -0.207 \\
\hline Viscosity & 0.392 & -0.158 & -0.012 & -0.054 & -0.025 & -0.066 & -0.166 & -0.080 & 0.037 & -0.190 & 0.242 & -0.001 & 0.032 & -0.096 & -0.044 & 0.502 & -0.092 & -0.506 & -0.394 \\
\hline Sulfur & 0.270 & 0.096 & 0.098 & 0.150 & 0.034 & -0.305 & 0.356 & 0.467 & -0.134 & 0.182 & -0.112 & -0.572 & 0.128 & -0.115 & -0.025 & 0.047 & -0.151 & -0.029 & 0.015 \\
\hline Aromatics & 0.206 & 0.113 & -0.203 & -0.251 & -0.372 & 0.123 & 0.347 & -0.080 & 0.013 & -0.091 & -0.514 & 0.140 & 0.203 & 0.393 & 0.169 & -0.013 & -0.114 & -0.136 & -0.111 \\
\hline IBP & 0.215 & -0.233 & -0.137 & 0.051 & -0.082 & -0.265 & -0.500 & -0.001 & -0.033 & 0.546 & -0.367 & 0.062 & -0.030 & 0.176 & -0.219 & 0.079 & 0.145 & 0.075 & 0.023 \\
\hline T10 & 0.358 & -0.172 & -0.151 & -0.046 & 0.014 & -0.257 & -0.195 & -0.104 & 0.086 & -0.190 & 0.132 & 0.082 & 0.079 & -0.078 & 0.132 & -0.401 & -0.612 & 0.141 & 0.224 \\
\hline T50 & 0.396 & -0.081 & 0.102 & -0.038 & 0.026 & -0.001 & -0.029 & -0.019 & 0.006 & -0.357 & 0.149 & -0.135 & 0.113 & 0.332 & 0.063 & 0.250 & 0.423 & 0.333 & 0.420 \\
\hline T90 & 0.305 & -0.148 & 0.185 & 0.051 & 0.033 & 0.481 & 0.168 & -0.033 & -0.163 & 0.046 & 0.026 & 0.114 & 0.161 & -0.061 & -0.682 & -0.208 & -0.070 & 0.028 & 0.002 \\
\hline FBP & 0.298 & $\begin{array}{l}-0.154 \\
-0.150\end{array}$ & 0.170 & 0.052 & 0.082 & 0.441 & 0.083 & -0.041 & -0.170 & 0.413 & 0.020 & 0.101 & -0.214 & -0.156 & 0.579 & 0.115 & -0.077 & 0.048 & 0.096 \\
\hline Oxygen & -0.014 & 0.179 & 0.521 & -0.349 & -0.166 & -0.069 & -0.161 & 0.012 & -0.029 & 0.029 & 0.020 & 0.015 & 0.076 & 0.011 & 0.000 & 0.144 & -0.209 & 0.525 & -0.410 \\
\hline Tot Cet $\wedge 2$ & -0.165 & -0.336 & -0.031 & -0.263 & 0.128 & -0.044 & -0.066 & 0.127 & -0.682 & -0.347 & -0.290 & -0.026 & -0.253 & -0.078 & -0.017 & 0.064 & -0.064 & -0.005 & 0.038 \\
\hline Oxygen $\wedge 2$ & -0.017 & 0.177 & 0.517 & -0.341 & -0.179 & -0.059 & -0.203 & 0.018 & -0.017 & 0.074 & -0.005 & -0.032 & 0.010 & -0.001 & 0.007 & -0.196 & 0.055 & -0.529 & 0.423 \\
\hline TCet $x$ CImp & -0.011 & 0.189 & -0.382 & -0.396 & 0.087 & -0.010 & -0.013 & 0.110 & -0.402 & 0.297 & 0.476 & 0.073 & 0.363 & 0.110 & 0.042 & -0.065 & 0.093 & -0.008 & -0.024 \\
\hline Sulfur $\wedge 2$ & -0.230 & -0.251 & -0.150 & -0.162 & -0.143 & 0.412 & -0.245 & -0.163 & 0.144 & 0.038 & 0.009 & -0.695 & 0.150 & 0.072 & 0.023 & 0.018 & -0.155 & 0.009 & 0.019 \\
\hline CImp $\times$ Sulf & -0.094 & -0.211 & 0.136 & 0.073 & -0.183 & -0.356 & 0.373 & -0.703 & -0.238 & 0.178 & 0.150 & -0.120 & 0.007 & 0.016 & -0.042 & 0.024 & 0.027 & 0.010 & 0.031 \\
\hline Dens $x$ Sulf & -0.096 & -0.505 & 0.065 & -0.200 & -0.051 & -0.097 & 0.132 & 0.152 & 0.234 & -0.001 & -0.113 & 0.170 & 0.499 & -0.448 & 0.136 & -0.039 & 0.269 & 0.039 & -0.010 \\
\hline Sulf $\times$ Arom & -0.085 & -0.420 & 0.020 & -0.283 & -0.092 & -0.069 & 0.287 & 0.322 & 0.280 & 0.147 & 0.296 & 0.074 & -0.431 & 0.369 & -0.117 & -0.009 & -0.092 & -0.010 & -0.002 \\
\hline Eigenvalues & 5.317 & 2.713 & 2.173 & 1.888 & 1.645 & 1.143 & 0.953 & 0.785 & 0.548 & 0.527 & 0.461 & 0.260 & 0.175 & 0.163 & 0.096 & 0.064 & 0.048 & 0.023 & 0.017 \\
\hline Pct Variance & 27.985 & 14.281 & 11.439 & 9.939 & 8.659 & 6.018 & 5.014 & 4.131 & 2.884 & 2.772 & 2.425 & 1.366 & 0.919 & 0.860 & 0.504 & 0.339 & 0.250 & 0.124 & 0.092 \\
\hline Cumulative Pct & 27.985 & 42.266 & 53.706 & 63.645 & 72.304 & 78.322 & 83.336 & 87.467 & 90.350 & 93.122 & 95.547 & 96.913 & 97.832 & 98.692 & 99.195 & 99.534 & 99.785 & 99.908 & 100.000 \\
\hline \multicolumn{20}{|c|}{ PCA Eigen variables in physical units: Deltas } \\
\hline & 1 & 2 & 3 & 4 & 5 & 6 & 7 & 8 & 9 & 10 & 11 & 12 & 13 & 14 & 15 & 16 & 17 & 18 & 19 \\
\hline TotCetane & 0.781 & -0.503 & 1.059 & -0.903 & 4.165 & -0.371 & 0.088 & -1.056 & 0.616 & -0.019 & -0.791 & -0.985 & 0.174 & 1.700 & 0.807 & -2.367 & 1.146 & -0.733 & -2.210 \\
\hline CetImprv & 0.216 & 0.952 & -0.785 & -2.069 & 1.706 & -0.120 & 0.766 & -0.982 & 1.159 & 0.518 & -0.867 & -0.064 & -0.634 & -1.259 & -0.963 & 1.561 & -0.577 & 0.377 & 1.256 \\
\hline Density & 0.005 & 0.002 & -0.003 & -0.003 & -0.005 & -0.000 & -0.000 & -0.001 & 0.000 & -0.001 & 0.001 & -0.003 & -0.006 & -0.006 & -0.000 & -0.005 & 0.006 & 0.001 & -0.003 \\
\hline Viscosity & 0.247 & -0.099 & -0.008 & -0.034 & -0.016 & -0.041 & -0.105 & -0.051 & 0.023 & -0.119 & 0.152 & -0.001 & 0.020 & -0.061 & -0.028 & 0.316 & -0.058 & -0.319 & -0.248 \\
\hline Sulfur & 44.286 & 15.732 & 16.088 & 24.640 & 5.603 & -49.988 & 58.499 & 76.567 & -22.046 & 29.876 & -18.319 & -93.863 & 21.046 & -18.833 & -4.138 & 7.750 & -24.706 & -4.714 & 2.490 \\
\hline Aromatics & 1.681 & 0.924 & -1.657 & -2.050 & -3.038 & 1.004 & 2.840 & -0.651 & 0.108 & -0.742 & -4.205 & 1.143 & 1.659 & 3.211 & 1.380 & -0.105 & -0.931 & -1.115 & -0.906 \\
\hline IBP & $\begin{array}{r}1.001 \\
11.868\end{array}$ & $\begin{array}{r}. .244 \\
-12.880\end{array}$ & $\begin{array}{l}-1.051 \\
-7.587\end{array}$ & 2.813 & -4.507 & $\begin{array}{r}1.004 \\
-14.670\end{array}$ & $\begin{array}{r}2.040 \\
-27.668\end{array}$ & $\begin{array}{l}-0.051 \\
-0.072\end{array}$ & $\begin{array}{r}-1.851 \\
-1.85\end{array}$ & 30.170 & $\begin{array}{l}-4.285 \\
-20.282\end{array}$ & $\begin{array}{l}3.403 \\
3.403\end{array}$ & $\begin{array}{r}1.059 \\
-1.642\end{array}$ & $\begin{array}{l}9.211 \\
9.736\end{array}$ & $\begin{array}{r}1.500 \\
-12.119\end{array}$ & $\begin{array}{r}-0.105 \\
4.358\end{array}$ & $\begin{array}{r}-0.011 \\
8.014\end{array}$ & $\begin{array}{r}-1.167 \\
4.167\end{array}$ & $\begin{array}{r}-0.906 \\
1.246\end{array}$ \\
\hline $\begin{array}{l}\text { IBP } \\
\text { T10 }\end{array}$ & $\begin{array}{l}11.868 \\
13.843\end{array}$ & $\begin{array}{l}-12.8800 \\
-6.645\end{array}$ & $\begin{array}{l}-1.581 \\
-5.844\end{array}$ & $\begin{array}{r}2.813 \\
-1.770\end{array}$ & $\begin{array}{r}-4.501 \\
0.540\end{array}$ & $\begin{array}{l}-14.610 \\
-9.947\end{array}$ & $\begin{array}{r}-21.668 \\
-7.543\end{array}$ & $\begin{array}{l}-0.012 \\
-4.017\end{array}$ & $\begin{array}{r}-1.8511 \\
3.346\end{array}$ & $\begin{array}{l}30.170 \\
-7.352\end{array}$ & $\begin{array}{r}-20.282 \\
5.128\end{array}$ & $\begin{array}{l}3.403 \\
3.158\end{array}$ & $\begin{array}{r}-1.642 \\
3.045\end{array}$ & $\begin{array}{r}9.136 \\
-3.030\end{array}$ & $\begin{array}{r}-12.119 \\
5.106\end{array}$ & $\begin{array}{r}4.358 \\
-15.505\end{array}$ & $\begin{array}{r}8.014 \\
-23.705\end{array}$ & $\begin{array}{l}4.167 \\
5.441\end{array}$ & $\begin{array}{l}1.246 \\
8.669\end{array}$ \\
\hline T50 & 13.442 & -2.745 & 3.469 & -1.285 & 0.892 & -0.050 & -0.991 & -0.653 & 0.187 & -12.106 & 5.046 & -4.575 & 3.824 & 11.256 & 2.134 & 8.491 & 14.354 & 11.310 & 14.248 \\
\hline T90 & 10.659 & -5.177 & 6.449 & 1.772 & 1.163 & 16.821 & 5.880 & -1.170 & -5.707 & 1.608 & 0.918 & 3.968 & 5.627 & -2.138 & -23.825 & -7.272 & -2.463 & 0.979 & 0.054 \\
\hline FBP & 9.948 & -5.137 & 5.656 & 1.722 & 2.734 & 14.693 & 2.780 & -1.372 & -5.664 & 13.785 & 0.655 & 3.384 & -7.138 & -5.190 & 19.310 & 3.837 & -2.572 & 1.594 & 3.218 \\
\hline Oxygen & -0.006 & 0.081 & 0.236 & -0.158 & -0.075 & -0.031 & -0.073 & 0.006 & -0.013 & 0.013 & 0.009 & 0.007 & 0.035 & 0.005 & 0.000 & 0.065 & -0.094 & 0.238 & -0.186 \\
\hline Tot Cet $\wedge 2$ & -0.259 & -0.529 & -0.049 & -0.414 & 0.202 & -0.070 & -0.104 & 0.200 & -1.074 & -0.547 & -0.456 & -0.040 & -0.398 & -0.123 & -0.027 & 0.101 & -0.100 & -0.007 & 0.059 \\
\hline Oxygen $\wedge 2$ & -0.133 & 1.417 & 4.145 & -2.732 & -1.437 & -0.477 & -1.626 & 0.146 & -0.134 & 0.592 & -0.042 & -0.256 & 0.080 & -0.009 & 0.052 & -1.573 & 0.442 & -4.234 & 3.392 \\
\hline TCet $x$ CImp & -0.010 & 0.180 & -0.365 & -0.378 & 0.083 & -0.009 & -0.012 & 0.105 & -0.384 & 0.284 & 0.455 & 0.070 & 0.346 & 0.105 & 0.040 & -0.062 & 0.089 & -0.008 & -0.023 \\
\hline Sulfur $\wedge 2$ & -0.261 & -0.286 & -0.171 & -0.184 & -0.162 & 0.468 & -0.279 & -0.185 & 0.163 & 0.044 & 0.011 & -0.790 & 0.171 & 0.082 & 0.027 & 0.020 & -0.176 & 0.010 & 0.021 \\
\hline CImp $x$ Sulf & -0.096 & -0.217 & 0.140 & 0.075 & -0.188 & -0.367 & 0.383 & -0.723 & -0.245 & 0.184 & 0.154 & -0.124 & 0.008 & 0.016 & -0.043 & 0.024 & 0.028 & 0.010 & 0.032 \\
\hline Dens $x$ Sulf & -0.120 & -0.632 & 0.082 & -0.250 & -0.063 & -0.121 & 0.166 & 0.190 & 0.293 & $\begin{array}{l}-0.001 \\
-0.01\end{array}$ & -0.142 & 0.213 & 0.624 & -0.560 & 0.170 & $\begin{array}{l}-0.049 \\
\end{array}$ & 0.336 & 0.049 & $\begin{array}{l}-0.013 \\
-0.013\end{array}$ \\
\hline Sulf $\times$ Arom & -0.102 & -0.506 & 0.024 & -0.341 & -0.110 & -0.083 & 0.346 & 0.389 & 0.338 & 0.178 & 0.356 & 0.089 & -0.520 & 0.445 & -0.141 & -0.011 & -0.111 & -0.012 & -0.003 \\
\hline
\end{tabular}


Table B.2. PCR+ Regression Model for PM Emissions

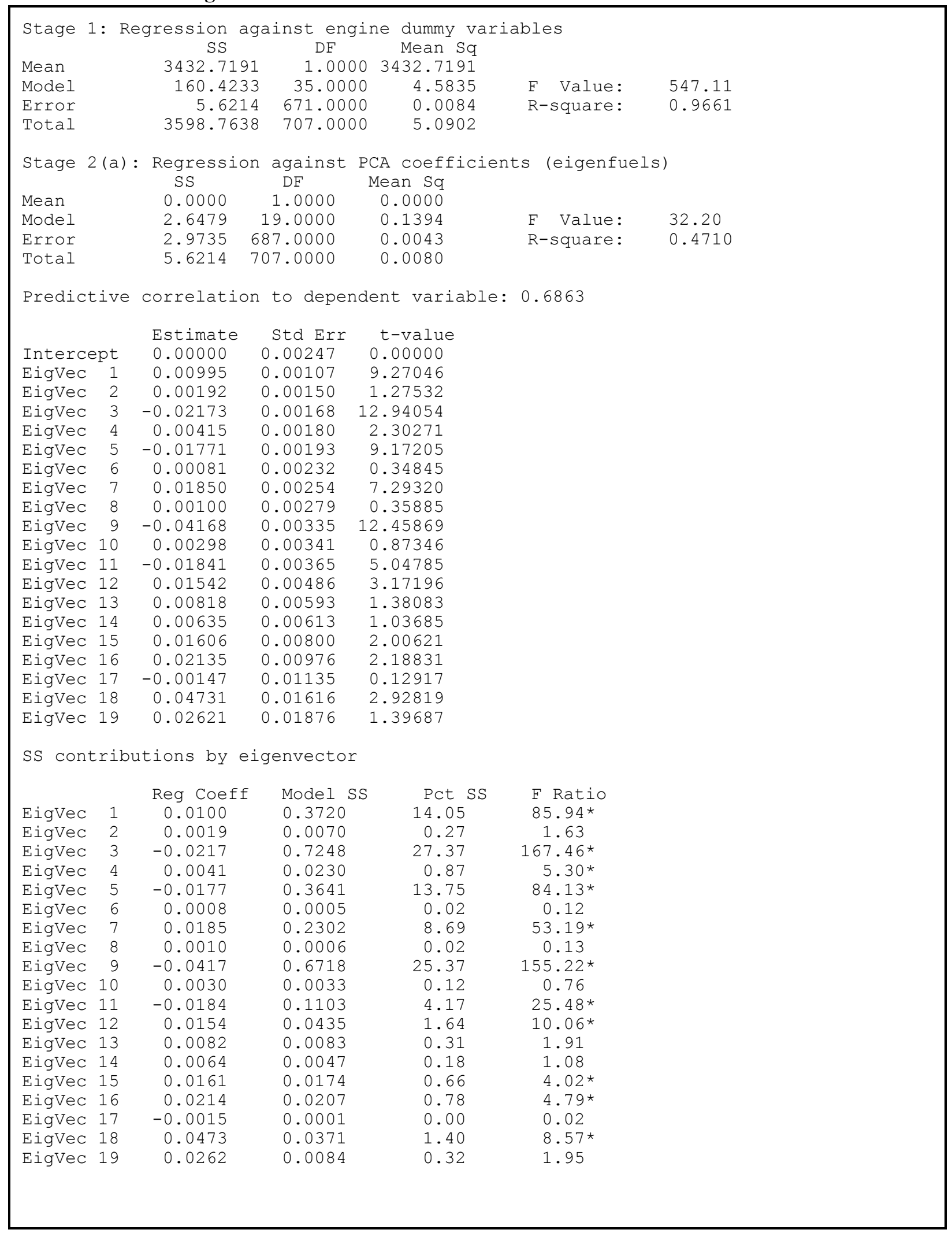


Table B.2. (Continued)

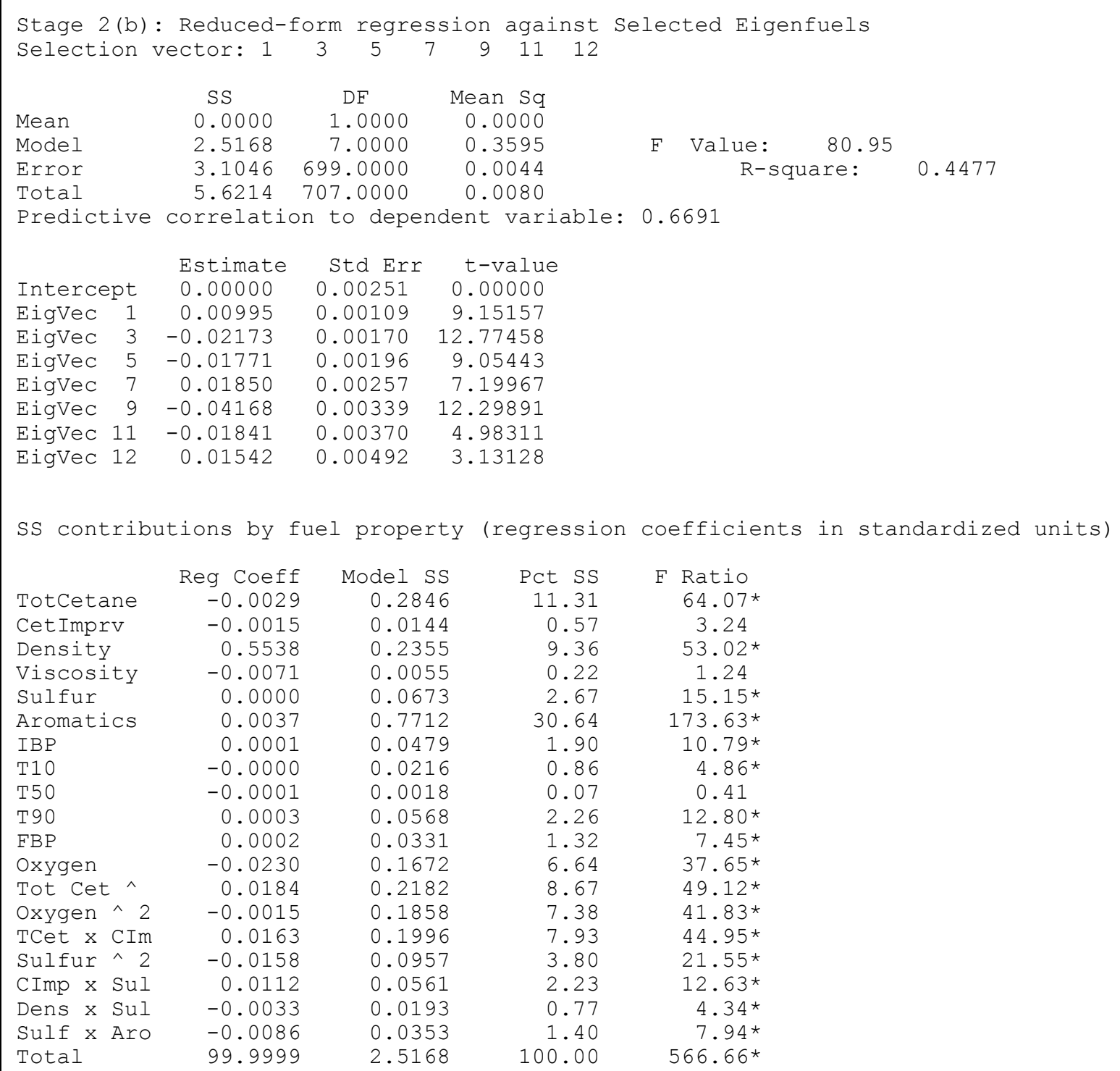



APPENDIX II-C

PEER REVIEW COMMENTS AND REPLY 



\section{Review of DRAFT Estimating Impacts of Diesel Fuel Reformulation with Vector-Based Blending, ORNL/TM-2002/225}

Reviewer: David Andress

David Andress \& Associates, Inc.

1108 Harriet Lane

Kensington, MD 20895

November 6, 2002

To formulate policy recommendations and comment on proposed regulations concerning reformulated diesel fuels, the Department of Energy requires high quality analyses of cost and produceability impacts associated with potential diesel fuel reformulations. The ORNL report does an excellent job of meeting that objective. The study premises and expository material are well laid out, and the conclusions are presented in a straightforward and coherent manner. The authors did a good job of the describing the study limitations, which are inevitable given the current limitations in emissions data and the early stage of the public debate on reformulated diesel fuels.

The study consists of two separate research activities: a set of refinery analyses and estimates of NOx and PM emission models for diesel fuels. The ORNL-RYM model, which was used for the refinery analyses, has been used in many important DOE and EPA studies and has been extensively peer reviewed by industry and other stakeholders. On the other hand, the eigenfuel approach to estimating emission models is relatively new and still evolving. Accordingly, most of our comments pertain to the eigenfuel analysis and include some suggestions for additional exploratory data analysis.

Developing an emissions model for diesel fuels is a challenging effort for a number of reasons including the acknowledged limitations in the available data. An earlier EPA attempt, for example, was concluded after receiving public comments without a producing a model recommendation. The eigenfuel model described in the ORNL study presents a viable candidate for public review. We note that API commented on the fact that the EPA database used to develop the emissions model did not include emissions data for diesel engines with advanced emission controls. In particular, API noted that the correlation of NOx emissions with cetane was different in engines equipped with exhaust gas recirculation (EGR) from those without EGR. The EPA database did not contain data on engines with EGR. Although the API comment is true, we believe the ORNL approach is correct. If the EPA data base had included emissions data on both engines with and without EGR, they would have to be separated prior to performing a regression analysis. Comparison can be made to the California Predictive Model in which separate regression models are fit to different vehicle technology classes.

One of the report's conclusions is that the eigenfuel-based emissions approach shows increasing benefits as recipe specifications are relaxed. However, the economic benefit for the sensitivity case that was analyzed was comparatively small. We offer the following observations:

4. California prescribes a recipe for diesel fuel properties, but allows the refiners to use alternative formulations provided independent testing verifies the emissions from the alternative formulation are equal to or less than those of the reference fuel. According to material on the CARB web site, most refiners in California use alternative diesel formulations that are certified to meet emission standards through independent emissions tests. This suggests an economic benefit exists for blending flexibility, and the eigenfuel-based emissions methodology could confirm this for existing diesel fuel standards. Whether there is an economic benefit of any significance for the stricter 2006 California diesel fuel regulations has not been investigated. Since California tends to lead the nation in adopting environmental standards, a future study could examine this issue. Such a study could provide input for establishing a diesel emissions 
model for use in California and Texas. Using an emissions model to certify diesel fuels, rather than having to subject alternative diesel fuel formulations to independent testing, would be valuable to refiners.

5. It is not surprising that "vehicle performance" and "emissions reduction" specifications in RYM produce similar results. Cetane control, aromatics reduction, and specific gravity are key fuel components that contribute to emissions reductions of both NOx and PM. The contribution to emissions reduction from specific gravity is small. As noted in the ORNL report, specific gravity is correlated with aromatic content and cetane. We can infer that not much leeway beyond aromatic and cetane control exists in the current RYM representation to reduce NOx and PM emissions. Given the way the emission equations are represented in RYM, it would appear that even with somewhat less stringent requirements on NOx and PM emissions, increases in cetane and reductions in aromatic content would still be needed.

6. The ORNL study only considered NOx and PM emissions, which are the primary emissions of concerns for diesel fuels. A complete analysis would also include $\mathrm{HC}, \mathrm{CO}$, and toxic emissions. The Auto Alliance World Wide Fuel Charter includes data that show that substantial reductions in $\mathrm{HC}$ and $\mathrm{CO}$ emissions occur as cetane increased, and that the percentage reductions in $\mathrm{HC}$ and $\mathrm{CO}$ emissions are greater than those of NOx and PM emissions. This could further constrain refiner flexibility in meeting emission reductions in the existing diesel fleet that are equivalent to the Auto Alliance recipe proposal.

7. As the ORNL study notes, cetane has an effect on cold startability. As emissions are higher when engines are cold, cold startability could have a significant effect on diesel emissions. The relative difference between emissions from hot and cold engines may be greater for engines with advanced emission control technologies.

8. Finally, we note the ongoing tussle between the auto and oil industries about how to implement technologies to reduce vehicular emissions and how to divide up the responsibility between the auto and oil industries. The auto industry would like greater control in setting fuel recipe standards. The following quote from the World Wide Fuel Charter illustrates their argument:

Production diesel engines are set to a standard density which determines the amount of fuel injected. The (volumetric) injection quantity is a control parameter for other emission control systems like the exhaust gas recirculation (EGR). Variations in fuel density therefore result in non-optimal EGR-rates for a given load and speed point in the engine map and, as a consequence, influence the exhaust emission characteristics.

Part II of the report, which describes the derivation of eigenfuels, explains that a conventional regression analysis was used to estimate the emission equations that are input to ORNL-RYM. The methodology was: (1) apply an eigenvalue analysis to the full data set; (2) use the results of Step (1) to generate a synthetic data set limited to the domain of interest (upper and lower value constraints on fuel parameters); and (3) apply a standard regression analysis to the synthetic data step generated in Step (2). The use of the synthetic data set appears similar to a technique called random balancing. The reason for using random balancing is to simplify the regression model, since some of the quadratic or interactive that were statistically significant in the original regression may not be in the new regression. Nonetheless, we wonder why a standard regression analysis was applied to the synthetic data in lieu of using another eigenfuel analysis and/or what the advantage was in using the eigenfuel approach for the first regression. 
Part II notes the eigenvector and regression analyses are mathematically equivalent, i.e., one representation can be transformed into the other. This raises the obvious question of the advantage of using one approach over the other. We believe that a comparison between a pure eigenfuel approach and a pure regression approach would be useful to assess whether the eigenfuel approach described in Part II provides additional benefit.

In reviewing documentation describing how regression models were selected for regulatory compliance models, we observed cases where a final regression model was selected from a set of regression models with similar R squares. This situation occurs when collinearity among some of the variables is present. We note that the selection process can appear somewhat arbitrary at times. However, a "good" selection process should incorporate an understanding of the physical processes.

We suggest exploring the use of a mixed effect regression model, as there seems to be a growing acceptance in the regulatory arena for this approach. California used a mixed effect regression analysis to estimate the Predictive Model equations. The California approach treats the fuels as the fixed effects and the vehicles as the random effects. The recently released EPA biodiesel emissions analysis also used a mixed effect model. EPA used a fixed effect regression analysis to estimate the Complex Model equations, and California has identified this as one the reasons why emission estimates produced by the Predictive and Complex models are not consistent.

On Pages S-5 and 1-2, a statement is made to the effect that the eigenfuel methodology results in economy of representation. However, the characterization used in ORNL-RYM is based on the original fuel properties (from page II-4 "to accommodate RYM, eigenvector models are transformed to fuel property models"), and so no economy of representation occurs in the context of the study.

Pages S-1 and 1-2, state that the eigenfuels provide a greater understanding of the patterns of variation needs to be explained in the text. In Part II, the eigenfuels are loosely interpreted in terms of the original fuel parameters, but no new insights are provided. For example, does a particular eigenfuel correspond to a group of fuel properties that are commonly controlled in the refinery proportionate to their coefficients in the eigenfuel? Part II notes that the analysis uncovered the statistical significance of some interactive terms, which was not previously recognized. Jerry noted that the EPA proposed model did not contain a cetane term. (I find this hard to understand considering the emphasis place on the relationship between cetane and emissions in the World Fuels Charter.) A comparison with the discontinued EPA effort and other studies would be useful in identifying insights gained from the eigenvector approach.

Page 1-1 states that emission benefits may be misrepresented if based on analysis of individual fuel properties. While this statement is true, it is not clear how the eigenfuel approach contributes to a better understanding of the problem. The eigenfuel representation in RYM is based on individual fuel properties.

An advantage that is claimed for the eigenfuel approach is that is provides a non arbitrary way of dealing with collinearity in the independent variables. However, the eigenvector approach merely shifts the collinearity problem from regression analysis to principle component analysis. Just as there can be several regression models with similar R squares using different subsets of the original variables, different orthogonal bases can be selected with much the same result. For example, the orthogonal basis in which a single vector explains all the regression variance is the one determined by a regression analysis. (The remaining orthogonal basis vectors can be selected arbitrarily.)

The eigenfuel methodology applied to estimate NOx and PM emissions is an interesting adaptation to the theoretical principal component analysis. Quadratic and interactive terms are introduced because of a positive correlation with NOx or PM emissions, not to explain patterns in the underlying fuel property 
space. Another avenue that might be interesting to explore is to perform a principle component analysis without added quadratic and interactive terms, and then determine if any quadratic and interactive eigenfuels need to be added.

Page 1-13 says the "most important eigenfuels are clearly related to refinery processing and blending". Part II does not fully support this assertion. The interpretation of the eigenfuels was in terms of the original fuel components, i.e., those physical components with the largest contribution to the eigenfuels. Although the eigenfuels may provide additional physical insights, the document did not identify any. It should be noted that the choice of the eigenfuels is related to both component scaling and whether normalization transformations (e.g., differences from mean) are used. This is an area for further research, as the choice of scaling can help in formulating a physical or economic interpretation of the eigenfuels.

The application of the eigenfuels in Part II was to estimate NOx and PM emissions, i.e., to gain insights into the relationship between refinery processing and blending and NOx and PM emissions. In that process, the selection of which quadratic and interactive terms to include in each analysis depends on their respective correlation with NOx or PM emissions. The following table lists the eigenvector numbers for the NOx and PM emissions with the greatest contributions to the respective regression analyses.

\begin{tabular}{|c|c|c|c|c|}
\hline \multirow{2}{*}{$\begin{array}{l}\text { Eigenvector } \\
\text { number }\end{array}$} & \multicolumn{2}{|c|}{ NOx Emissions } & \multicolumn{2}{|c|}{ PM Emissions } \\
\hline & $\begin{array}{l}\text { Pct. variance } \\
\text { contribution of } \\
\text { eigenvector }\end{array}$ & $\begin{array}{l}\text { Pct. variance } \\
\text { contribution for } \\
\text { regression (part } \\
\text { explained by } \\
\text { regression } \\
\text { analysis) }\end{array}$ & $\begin{array}{l}\text { Pct. variance } \\
\text { contribution of } \\
\text { eigenvector }\end{array}$ & $\begin{array}{l}\text { Pct. variance } \\
\text { contribution for } \\
\text { regression (part } \\
\text { explained by } \\
\text { regression analysis }\end{array}$ \\
\hline 1 & 31 & 22 & 28 & 14 \\
\hline 2 & 14 & 32 & 14 & 0 \\
\hline 3 & 11 & 0 & 11 & 27 \\
\hline 4 & 9 & 16 & 10 & 0 \\
\hline 5 & 7 & 2 & 9 & 14 \\
\hline 9 & 3 & 14 & 4 & 25 \\
\hline
\end{tabular}

As can be seen in the above table, an argument can be made that the most significant eigenvectors are (for the most part) most correlated to NOx emissions, but not for PM emissions.

The statement about the economy of representation for the eigenvectors may be true, but needs to be demonstrated. For example, how many eigenfuels are needed to explain X percent of the regression variation versus how many fuel properties are needed. As the regression analysis in Part II used all the eigenfuels, there was no economy of representation in that analysis. In addition, we wonder why terms whose coefficients were not statistically significant were retained in the final regression models. Table 4.2, for example, shows regression coefficients for T50 and FBD although they are not statistically significant at the 0.05 level (Table 3.1).

Fig. 1 (1-15) does not present a balanced comparison between regressions using the fuel properties and the eigenfuels. In both cases, there are 12 variables that can be used in the regression analysis. That is, 
there 4,095 different regression models for cases. For the eigenvector regressions, only one of the possible 4,095 different regression models was selected using a standard stepwise regression approach ( looking at the sequential improvement in the R-square statistic). The same methodology should be applied to the used for regressions based on the fuel properties. This would provide a more balanced comparison between regression analyses based on fuel properties versus eigenfuels. It should also be noted that the discussion on page I-13 and the analysis in Part II differ in that quadratic and cross product terms are included in Part II. It appears that the discussion in Part I is based on an earlier eigenfuel analysis.

Statistical model building is an art, and different analytic representations may lend themselves in varying degrees to understanding the underlying physical phenomena. To this extent, the use of the eigenfuels approach may show patterns that were not previously understood. The value may be in understanding how fuel properties are jointly varied in the refinery blending operation, i.e., as a pattern recognition tool. However, we note that a recognized weakness in principle component analysis is in interpreting the eigenvectors, and the interpretations can be affected by component scaling. Other papers by the authors have explored this concept in more detail, and they may have provided additional insights in this area. Nonetheless, we endorse the eigenfuel approach as a useful tool for exploratory analysis and have provided some thoughts for additional explanatory research. We want to acknowledge the quality of the presentation in Part II, which included a good list of caveats and discussion of data limitations. 


\section{Author Reply to Review of DRAFT Estimating Impacts of Diesel Fuel Reformulation with Vector- Based Blending, ORNL/TM-2002/225}

Dave Andress:

Thanks very much for your careful and thoughtful review of our RFD report. We will revise the report to address your comments and incorporate your editorial suggestions. We believe that several of your questions are answered in our previous publications (which I mailed to you last week) on the eigenfuel methodology, and we may need to use more specific literature citations in our RFD report. Regarding your specific comments:

Your page 3: “... we believe that a comparison between a pure eigenfuel approach and a pure regression approach would be useful to assess whether the eigenfuel approach described in Part II provides additional benefit." See ORNL/TM-2002/16, Section 3.4, among others.

Your page 3: “... we wonder why a standard regression analysis was applied to the synthetic data in lieu of using another eigenfuel analysis and/or what the advantage was in using the eigenfuel approach for the first regression." This is done solely as a "curve fitting" tool to give us an equivalent model stated in terms of the individual fuel properties that could be implemented within RYM. That is, the emission analysis was conducted in the vector space using eigenfuels as the explanatory variables. Having developed the models, we needed to transform to equations written using the fuel properties. This can actually be done algebraically (with some effort); the random balance regression approach was chosen as a practical approach for making the transformation.

Your page 3:"... This raises the obvious question of the advantage of using one approach over the other." The advantage of the eigenfuel approach is that it will not be "fooled" by the aliasing existing among the individual properties, while conventional methods can misidentify the influential variables. See ORNL/TM-2002/16, Section 3 for a full discussion of this.

Your page 3: “ ... a statement is made to the effect that the eigenfuel methodology results in economy of representation. However, the characterization used in ORNL-RYM is based on the original fuel properties, and so no economy of representation occurs in the context of the study." And your page 5: "... The statement about economy of representation may be true, but needs to be demonstrated." In a computational sense -- counting the number of variables -- this is true, but computational power is cheap today. The reduction in dimensionality comes in the fact that we identified 6 (vector) fuel factors influencing NOx emissions and 7 (vector) fuel factors influencing PM emissions, out of variable spaces that encompassed 17 and 19 terms, respectively. We need all of the properties to describe the vectors, but there are only 6 and 7 vector features influencing NOx and PM emissions.

Your page 4: " ... does a particular eigenfuel correspond to a group of fuel properties that are commonly controlled in the refinery to proportionate to their coefficients in the eigenfuel?" See ORNL/TM-2002/16, Section 5. Eigenfuels are related (some more clearly than others) to blendstocks, implying a "macro" level of control using blendstock proportions, rather than property controls. We are not suggesting abandonment of property controls needed for trimming product quality.

Your page 4: "A comparison with the discontinued EPA effort and other studies would be useful in identifying insights gained from the eigenvector approach." See ORNL/TM-2002/16, Table 5.1, Sections 2.2 through 3.5 .

Your page 4: "Page 1-13 says the "most important eigenfuels are clearly related to refinery processing and blending."” See ORNL/TM-2002/16, Table 5.1 
Your page 4: “... emission benefits may be misrepresented if based on analysis of individual fuel properties." While the emission model in RYM is written in terms of individual fuel properties, as a matter of computation convenience, it was not developed using an analysis based on individual fuel properties. It was developed from an analysis that used eigenvectors and therefore avoided the problems that aliasing of variables can introduce.

Your page 4: "However, the eigenvector approach merely shifts the collinearity problem from regression analysis to principal component analysis." The collinearity problem is dealt with and resolved in PCA, which is something that conventional regression analysis cannot really achieve. When the number and identity of the explanatory variables is constant, there is only one orthogonal basis for the data set and that is the one identified by PCA. As you noted, the work did undertake a novel effort to identify vector bases that are efficient for describing fuel effects on emissions.

Your page 4: "It should be noted that the choice of eigenfuels is related to both component scaling and whether normalization transformations are used." See ORNL/TM-2002/16, Section 5 for support on the interpretation of eigenfuels in refining terms. Additional work on formulating physical interpretations of the eigenfuels would be beneficial.

Your page 5: "As can be seen in the above table, an argument can be made that the most significant eigenvectors are (for the most part) most correlated to NOx emissions, but not for PM emissions." You cannot safely draw the conclusion from the table. The variables spaces differed for the NOx and PM models, so that the $\mathrm{n}^{\text {th }}$ eigenvector of the NOx space is not necessarily related to the $\mathrm{n}^{\text {th }}$ eigenvector of the PM space.

Your page 5: "As the regression analysis in Part II used all the eigenfuels, there was no economy of representation in that analysis." The procedure is to use all of the eigenfuels initially, and then to drop those that do not contribute to the regression explanatory power. Therein lies the economy. (In fact, you must retain all of the vectors initially, or you risk excluding features that could be important in the regression.) As to Table 4.2, you must remember that the final regression of synthetic data is done only for curve-fitting purposes and is not a statistical analysis that purports to identify the influential factors. Thus, T50 is needed in the reduced-form model because it is a component of eigenfuels that had significant influences on emissions. This is not to say that T50 by itself has a statistically significant influence on emissions. We don't believe that the fuel properties have individual causal relationships to emissions.

If you would like to discuss this further, perhaps we could have a three-way conference call. Let us know if additional clarification would be helpful.

Thanks again for your excellent review.

G.R. Hadder and R.W. Crawford 